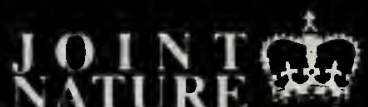

NATURE

CONSER VATION

COMMITTEE

\title{
Checklist of fish and invertebrates listed in the CITES appendices
}

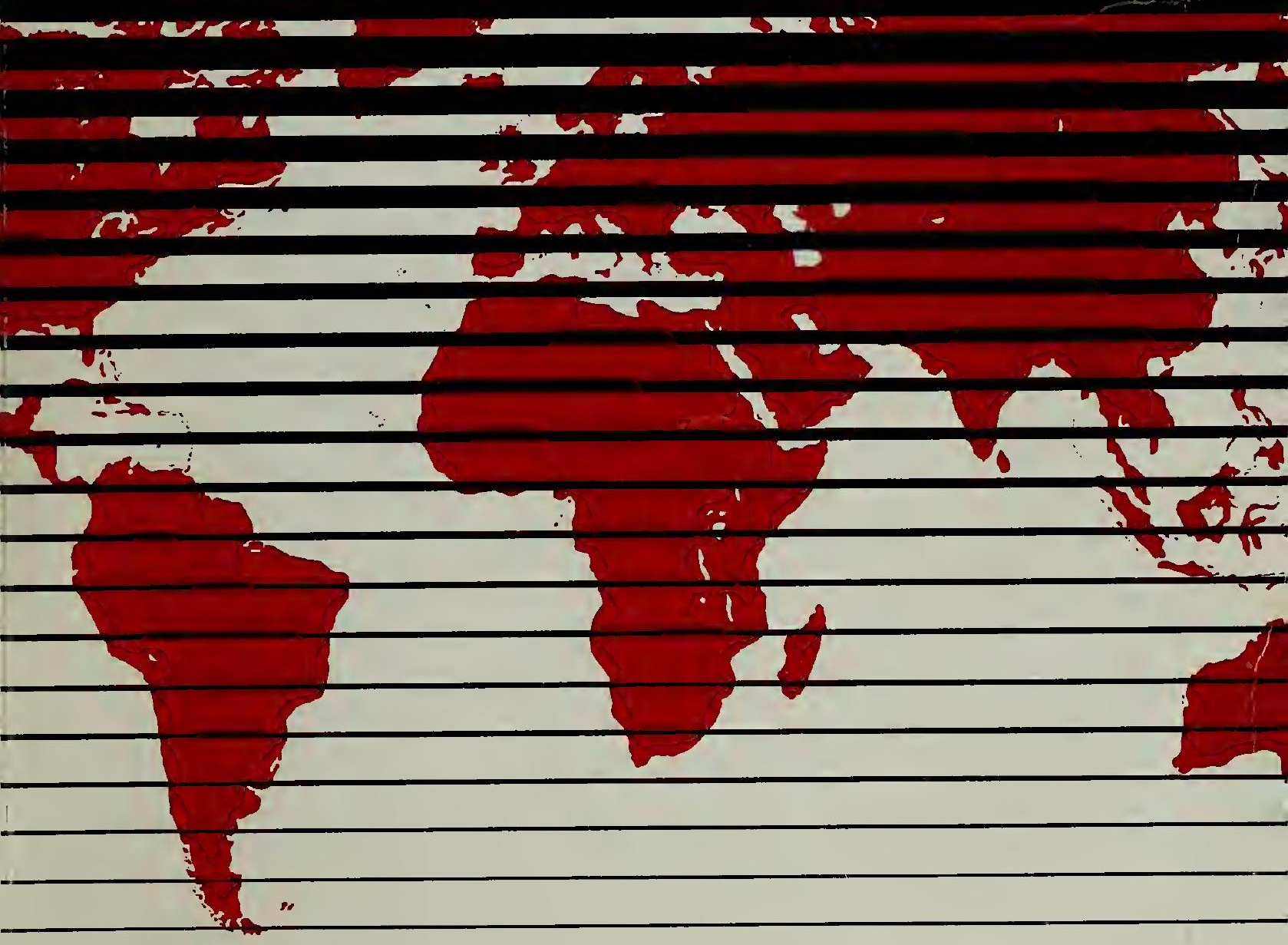


Digitized by the Internet Archive in 2010 with funding from UNEP-WCMC, Cambridge 

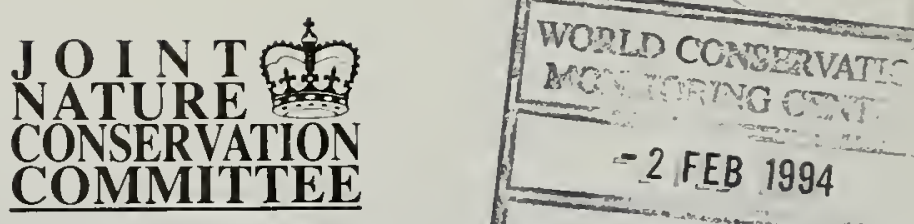

-2 FEB 1994

\title{
Checklist of \\ fish and invertebrates listed in the CITES appendices
}

\author{
compiled by the
}

World Conservation Monitoring Centre

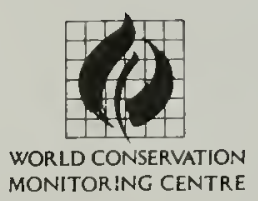

on behalf of the

United Kingdom Scientific Authority for Animals 
Prepared under contract from the Joint Nature Conservation Committee by the World Conservation Monitoring Centre, Cambridge, UK. Established in 1988 as a company limited by guarantee with charitable status, WCMC is managed as a joint-venture between the three partners in the World Conservation Strategy and its successor Caring For The Earth: JUCN - The World Conservation Union, UNEP - United Nations Environment Programme, and WWF - World Wide Fund for Nature. Its mission is to provide information on the status, security, management and utilisation of the world's biological diversity, and thereby to support conservation and sustainable development.

Published by: Joint Nature Conservation Committee

Copyright: 1993 Joint Nature Conservation Committee

1SBN: $\quad 1$ st edition published 1988 ISBN 0-86139-466-6

2nd edition published 1993 1SBN 1-873701-47-0

Citation: World Conservation Monitoring Centre (1993). Checklist of fish and invertebrates listed in the CITES appendices. Joint Nature Conservation Committee, Peterborough.

Available from: Further copies and companion volumes for other animal species available from:

Natural History Book Service Ltd

2 Wills Road

Totnes

Devon TQ9 5XN

United Kingdom

Telephone (from UK): $\quad 0803865913$

Telephone (from outside UK): +44803865913

The designations of geographical entities in this book, and the presentation of the material, do not imply the expression of any opinion whatsoever on the part of JNCC or WCMC concerning the legal status of any country, territory, or area, or of its authorities, or concerning the delimitation of its frontiers or boundaries. 


\section{Contents}

Preface

Acknowledgements

Introduction

Explanatory notes

Introductory references

Taxonomic list - Fish

CERATODIFORMES

Ceratodidae

COELACANTHIFORMES

Latimeriidae

\section{ACIPENSERIFORMES}

Acipenseridae

Polyodontidae

OSTEOGLOSSIFORMES

Osteoglossidae

CYPRINIFORMES

Cyprinidae

Catostomidae

\section{SILURIFORMES}

Schilbeidae

\section{PERCIFORMES}

Sciaenidae

REFERENCES - FISH

Taxonomic list - non-Coral Invertebrates

\section{LEPIDOPTERA}

Papilionidae

\section{ARANEAE}

Theraphosidae

\section{ARHYNCHOBDELLAE}

Hirudinidae

\section{VENEROIDA}

Tridacnidae

\section{UNIONOIDA}

Unionidae

\section{STYLOMMATOPHORA}

Achatinellidae

Camaenidae

Paryphantidae

\section{MESOGASTROPODA}

Strombidae

REFERENCES - NON-CORAL INVERTEBRATES

Taxonomic list - Black Corals

ANTIPATHARIA

Antipathidae

REFERENCES - BLACK CORALS

Taxonomic list - Hard Corals

MILLEPORINA

Milleporidae

STYLASTERINA

Stylasteridae

\section{HELIOPORACEA}

Helioporidae

\section{STOLONIFERA}

Tubiporidae

SCLERACTINIA

Astrocoeniidae 61

Pocilloporidae $\quad 62$

Acroporidae 65

Poriticiae $\quad 72$

Siderastreidae $\quad 76$

Agariciidae $\quad 79$

Micrabaciidae $\quad 82$

Fungiacyathidae $\quad 83$

Fungiidae $\quad 83$

Rhizangiidae 89

Oculinidae 90

Pectiniidae 93

Mussidae 95

Merulinidae 99

Faviidae 101

Trachyphylliidae $\quad 112$

Meandriniidae 112

Anthemiphylliidae $\quad 113$

Caryophylliidae $\quad 114$

Flabellidae $\quad 126$

Guyniidae $\quad 130$

Dendrophyllidae $\quad 130$

REFERENCES - HARD CORALS 137

INDEX AND SYNONYMY 141 



\section{Preface}

This checklist is of one of a series prepared for the United Kingdom's Scientific Authority for Animals to assist in implementing the Convention on International Trade in Endangered Species of Wild Fauna and Flora (CITES). Similar checklists are available for other animal taxa.

The aim of this volume is to provide a summary of basic information on all species of fish and invertebrates listed in CITES. Other volumes in the series have also included species listed in the IUCN Red List of Threatened Animals compiled by the World Conservation Monitoring Centre. However the Red List coverage is far from complete for fish and invertebrates so, in this volume, Red List categories are mentioned only for those species listed in CITES.

This volume, containing as it does English vernacular, scientific synonyms and a list of countries in which a taxon occurs, should provide a valuable aid to those assessing applications for CITES permits or with an interest in taxonomy, nomenclature and distribution of the groups included. For corals, this volume will provide the most up-to-date compilation of information on taxonomy and nomenclature, although this is inevitably incomplete especially at the species level.

The Joint Nature Conservation Committee is most grateful to the World Conservation Monitoring Centre for preparing this work on its behalf.

\section{Lord Selborne}

Chairman, Joint Nature Conservation Committee

Monkstone House

City Road

Peterborough

PE1 1JY

United Kingdom

\section{Acknowledgements}

This checklist was compiled by the World Conservation Monitoring Centre under contract F71-12-56 from the Joint Nature Conservation Committee. The authors for particular taxonomic groups were:

\author{
Helen Corrigan (fish) \\ Julie Hawkins (black corals) \\ Tim Inskipp (all taxa) \\ Helen Smith (non-coral invertebrates) \\ Elizabeth Wood (hard corals)
}

The volume builds on an earlier edition, and we gratefully acknowledge the numerous contributors to that edition, particularly the authors: Patricia C. Almada-Villela (compiler); N. Mark Collins (Insecta and Araneae); Tim Inskipp (all taxa) and Simon J. Moore (Antipatharia).

For their assistance in producing the current edition, the following WCMC staff are also thanked: Duncan Bennett, Esther Byford, Lorraine Collins, Mary Cordiner, John Easy, Vanessa Heywood, Richard Luxmoore, Julie Reay and Lindsay Simpson.

The Natural History Museum (London) provided access to information on taxonomy and distribution for material held in their collections.

David Morgan of the JNCC International Policy Branch is thanked for providing expert advice and guidance throughout.

Timothy Johnson

Head, Species Unit

World Conservation Monitoring Centre

219 Huntingdon Road

Cambridge

CB3 ODL

United Kingdom 


\section{Introduction}

In April 1991, the Nature Conservancy Council for England, Countryside Council for Wales and Scottish Natural Heritage acting together through the Joint Nature Conservation Committee were appointed by the Secretary of State for the Environment as the United Kingdom's Scientific Authority for Animals under the Convention on International Trade in Endangered Species of Wild Fauna and Flora (CITES). CITES regulates international trade in wild animals and plants and in products derived from them, to help to ensure their conservation on a worldwide scale.

The purpose of this publication is to provide a tax on list of fish and invertebrates included in Appendices I, II and III of the Convention on International Trade in Endangered Species of Wild Fauna and Flora (CITES). The other checklists in this series, covering mammals, birds, reptiles and amphibians, also include all species from each of these groups that are listed in the 1990 IUCN Red List of Threatened Animals. This checklist of fish and invertebrates differs in providing data only for those taxa listed on the CITES Appendices.

The rationale for treating fish and invertebrates differently from the other groups is that both are highly diverse, and neither has yet been comprehensively assessed for conservation status. The majority of species has therefore not been assigned an IUCN threat category and consequently the Red List contains only a small sample of the species from these groups that may be threatened. Despite this, the numbers of species of these two groups in the Red List are large: 713 fish and 1,977 invertebrates in the 1990 edition. Including all these in the checklist would greatly expand it without providing comprehensive species coverage of the groups involved.
Nevertheless, this second edition contains substantially more material than the first edition (Almada-Villela, 1988), reflecting changes to the CITES Appendices up to and including those made at the 8th Meeting of the Conference of the Parties in March 1992 (effective from 11 June 1992).

For background material on the rationale of the IUCN threat categories, readers are referred to IUCN (1990); for background on the workings of CITES, recommended works are Favre (1989) and Wijnstekers (1992). For information by country on the diversity and status of fish, invertebrates and other taxonomic groups, and for a general review of biodiversity, readers are referred to WCMC (1992).

In a publication of this nature, it is inevitable that users will discover entries that need correcting or updating. The publishers would be grateful if their attention could be drawn to these entries, and the World Conservation Monitoring Centre would be grateful to receive details of such changes so that they can be incorporated in the database from which this publication is produced. 


\section{Explanatory notes}

\section{Scientific names}

The taxonomic scope and sequence of orders follows the system adopted in the Appendices to CITES. For each taxon, the scientific name is given first (as listed in the CITES Appendices), with the most frequently used current synonyms in brackets. No standard nomenclature has been adopted by CITES for fish or invertebrates, with the exception of the birdwing butterflies, for which D'Abrera (1975) is followed.

\section{Fish}

Where the CITES nomenclature differs from that used in Nelson (1984) and Parenti (1981), the synonymy used by these works has been noted.

\section{Non-coral invertebrates}

The systematics of invertebrates has been the subject of numerous studies, and the nomenclature of the CITES listings may differ from the latest scientific reviews. In particular, there have been extensive revisions of the papilionid tribe Troidini and the pearly mussels of the family Unionidae. Miller (1987) revised the taxonomy of the Troidini, uniting all birdwing butterflies in the single genus Troides with two subgenera: Troides (including Troides, Ripponia and Ornithoptera) and Trogonoptera. Similarly, the Unionidae taxonomy was revised by Johnson (1978), reducing many of the Epioblasma species to synonymy and reinstating the generic name Plagiola. Other species in the same family were reviewed by Johnson (1980). To help address the problems caused by the complexity of the taxonomy of the North American molluscs, Turgeon et al (1988) produced a standard list of common and scientific names for all the fresh water molluscs of the United States and Canada. Wherever possible, the names used in this and the other taxonomic revisions discussed above are cross-referenced in the index.

\section{Black corals}

The latest major taxonomic revision of the Antipatharia was made by Opresko (1974): and forms the basis of this list, updated with more recent works. Although some workers have split the Antipatharia into a number of families, Opresko considered that there were only two families, the Antipathidae and the
Dendrobrachiidae. Opresko and Bayer (1991) subsequently reclassified the Dendrobrachiidae placing this monotypic family in the Gorgonacea. There are no known records of trade for this family and it is not included in this checklist.

\section{Hard corals}

Lists for genera and species of hermatypic reef corals occurring in the Indo-Pacific have been compiled by reference to recent publications by Veron $(1985,1986,1990 \mathrm{a}, \mathrm{b}, \mathrm{c})$ and Veron $e t$ al. (1976, 1977, 1980, 1982, 1984, 1988, 1989). These studies cover the central Indo-Pacific area and details of additional species occurring outside this area have been obtained, for example, from publications by Sheppard and Sheppard (1991) (Red Sea and Arabian Gulf), and Wells (1972, 1982, 1983) (Central and Eastern Pacific). Lists for genera and species of Western Atlantic reef corals have been compiled by reference to a number of key publications, for example by Cairns (1982a), Laborel (1970) and Wells and Lang (1973). Both reef and non-reef corals of the USA are listed in a booklet produced by the American Fisheries Society (1991). The taxonomy of deep water (ahermatypic) and temperate water scleractinian and stylasterid corals has been investigated in recent years principally by Cairns (1979, 1982b, 1983a,b,c, 1984, 1985, 1986a,b, 1987a,b, 1988, 1989, 1990, 1991a-e), Zibrowius (1973, 1974a,b, 1980) and Zibrowius et al. (1977. $1990,1992)$ and these references have been used extensively in the preparation of the checklist.

Reef genera distribution records have been provided as far as possible by country and references are given to relevant publications. Countries cited without reference numbers fall within the distribution range shown in general reviews e.g. Veron (1986) and Wood (1983). However, this assumption will not invariably be true and, in a few instances, the genus may not occur. For example, Brunei falls within the range for many genera and so is listed, but reefs in this country are poorly developed, so the listings may be incorrect. Conversely, some countries (e.g. Pakistan) are not listed, but may have coral communities. Exclusively deep water and deep/shallow water genera distribution (e.g. caryophylliids, dendrophylliids and stylasterids) are listed only by general geographic area. Many occur off the edge of the continental shelf. 


\section{English names}

The most widely used English common names appear on the line immediately following the scientific name and synonyms, below which the geographical range is indicated. The common names used have been taken from IUCN (1990) and from a number of standard reference works for fish and invertebrates of particular regions. Secondary common names have been included wherever this was considered useful, including non-English names commonly used by English speakers.

\section{Other information}

The three columns headed - CITES, RL and Ref, list the following information for each taxon.

\section{CITES}

I, or II in this column refers to the appendix on which the taxon is listed under the Convention on International Trade in Endangered Species of Wild Fauna and Flora. The letters (eq) after an Appendix II entry denote that the population is on Appendix II subject to an export quota.

\section{RL}

The status of the listed species is taken from the 1990 1UCN Red List of Threatened Animals. The Red List is a summary of information on all taxa known to be at risk. Additional information can be found in the IUCN Red Data Book Vol. 4: Pisces (Miller, 1977), the IUCN Invertebrate Red Data Book (Wells et al., 1983), Threatened Swallowtail Butterflies of the World (Collins and Morris, 1985), together with unpublished data sheets for North American fish taxa dated 1983, 1984 and 1985 held at the World Conservation Monitoring Centre.

Red List (RL) threat categories follow those given in the 1990 IUCN Red List of Threatened Animals, which also provides explanation of the categories:

\begin{tabular}{|c|c|}
\hline Ex & Extinct \\
\hline $\mathrm{E}$ & Endangered \\
\hline $\mathrm{V}$ & Vulnerable \\
\hline R & Rare \\
\hline Id & Indeterminate (given as "I" \\
\hline & $\begin{array}{l}\text { List, but modified here to a } \\
\text { with the CITES "l" entry) }\end{array}$ \\
\hline & Insufficiently known \\
\hline & Of special concern \\
\hline
\end{tabular}

CT Commercially threatened

- $\quad$ not listed: note this includes species which have not yet been evaluated for threatened status as well as those which have been evaluated and found not to be threatened.

\section{References}

The numbers in the right-hand column refer to entries in the reference list at the end of each section of the document. Many of these are general works relevant to more than one country or concerned with particular groups of species. Single country faunas and more specific references have generally been inserted in brackets after the appropriate country in the listings for geographical range.

\section{Geographical Range}

The geographical range of each taxon is given in terms of political units arranged alphabetically. Small island dependencies, are also listed alphabetically. Where appropriate, islands within groups are listed after a colon, e.g. Indonesia: Java. The same principle is applied whenever the distribution of a taxon is given in terms of units smaller than the country concerned.

Place-names and names of countries follow the Times Atlas (1990) and United Nations (1991).

It should be noted that, when a country is listed as being a range state of a species, the species may not occur throughout the country, and may even occur in only one or a few localities within the country.

Published records of distribution have been used. Many taxonomic works give the range of a taxon in terms of broad geographical areas, rather than of political units. Individual countries may have a relatively well-known and well-reported fish or invertebrate fauna, but others do not, and inevitably it has not been possible to access all reference works. These factors may occasionally have resulted in some of the geographical ranges given here being incomplete or inaccurate, although every effort has been made to prevent this. 


\section{Distribution notes}

A question mark '?' in front of a country indicates some uncertainty over the occurrence of the species in that country.

A country where the species is known to be extinct is denoted by '(ex)'; a country where there is still a possibility that the species survives (for instance because recent searches have been unsuccessful) is denoted as '(ex?)'. Range states where the species is introduced are denoted by '[ ]'. 


\section{Names of countries and dependent territories}

This checklist has been made as compact as possible by listing some names of countries and dependent territories in the shorter forms given by United Nations (1991). Within the text, Czechoslovakia and Yugoslavia are used to denote the geographic areas as recognised by United Nations (199I). However, changes to the political boundaries within the former USSR have been incorporated in the geographic descriptions.

Countries for which shorter forms of names are used and their shortened form:

$\begin{array}{ll}\text { Brunei Darussaiam } & \text { Brunei } \\ \text { Democratic People's Republic of Korea } & \text { D.P.R. Korea } \\ \text { Falkland Islands and Dependencies } & \text { Falkland Islands } \\ \text { Islamic Republic of Iran } & \text { Iran } \\ \text { Lao People's Democratic Republic } & \text { Lao P.D.R. } \\ \text { Libyan Arab Jamahiriya } & \text { Libya } \\ \text { Northern Mariana Islands } & \text { Northern Marianas } \\ \text { Republic of Korea } & \text { Korea Republic } \\ \text { Saint Vincent and the Grenadines } & \text { Saint Vincent } \\ \text { Syrian Arab Republic } & \text { Syria } \\ \text { United Republic of Tanzania } & \text { Tanzania } \\ \text { United Kingdom of Great Britain and Northern Ireland } & \text { United Kingdom } \\ \text { United States of America } & \text { USA }\end{array}$

In the text, parent countries of dependencies are omitted. These are listed below:

American Samoa, USA

Anguilla, United Kingdom

Aruba, Netherlands

Azores, Portugal

Bermuda, United Kingdom

Bouvet Island, Norway

British Antarctic Territory, United Kingdom

British Indian Ocean Territory, United Kingdom

British Virgin lslands, United Kingdom

Canary Islands, Spain

Canton and Enderbury lslands, Kiribati

Cayman Islands, United Kingdom

Channel Islands, United Kingdom

Christmas Island, Australia

Cocos Keeling Islands, Australia

Cook 1slands, New Zealand

Falkland Islands, United Kingdom

Faeroe Islands, Denmark

Federated States of Micronesia, USA

French Guiana, France

French Polynesia, France

French Southern and Antarctic Territories, France

Galapagos Islands, Ecuador

Gibraltar, United Kingdom

Greenland, Denmark

Guadeloupe, France

Guam, USA

Guernsey, United Kingdom

Hawaiian Islands, USA

Heard and Macdonald Islands, Australia

Hong Kong, United Kingdom

Isle of Man, United Kingdom

Jersey, United Kingdom
Johnston Atoll, USA

Macao, Portugal

Macquarie Island, Australia

Madeira, Portugal

Marshall Islands, USA

Martinique, France

Mayotte, France

Montserrat, United Kingdom

Netherlands Antilles, Netherlands

New Caledonia, France

Niue, New Zealand

Norfolk Island, Australia

Northern Marianas, USA

Palau, USA

Panama Canal Zone, USA

Pitcairn Islands, USA

Puerto Rico, USA

Queen Maud Land, Norway

Réunion, France

Saint Helena, United Kingdom

Saint Pierre and Miquelon, France

Saint Vincent, United Kingdom

South Orkney Islands, United Kingdom

South Sandwich Islands, United Kingdom

South Georgia, United Kingdom

South Shetland Islands, United Kingdom

Svalbard and Jan Mayen Islands, Norway

Tokelau, New Zealand

Tristan da Cunha Islands, United Kingdom

Turks and Caicos lslands, United Kingdom Virgin Islands of the United States, USA

Wallis and Futuna, France 


\section{Introductory References}

Almada-Villela, P.C. 1988. Checklist of fish and inveriebrates listed in the CITES Appendices. Nature Conservancy Council, UK.

American Fisheries Society 1991. Common and scientific names of aquatic inverebrates from the United States and Canada: Cnidaria and Clenophora. American Fisheries Society, Bethesda, Maryland.

Cairns, S.D. 1979. The deep-water Scleractinia of the Caribbean Sea and adjacent waters. Studies on the Faune of Curoçao and other Caribbean Islands 57(180): 341pp.

Cairns, S.D. 1982a. Stony corals (Cnidaria: Hydrozoa, Scleractinia) of Carrie Bow Cay, Belize. pp 271-302, in K. Rützler and I.G Macintyre, eds., The Atlantic Barrier Reef Ecosystem at Carrie Bow Cay, Belize, 1: Structure and communities. Smithsonian Contributions to Marine Sciences 12: $539 \mathrm{pp}$

Cairns, S.D. 1982b. Antarctic and subantarctic Scleractinia. Antarctic Research Series 34: 1-74.

Cairns, S.D. 1983a. Antarctic and subantarctic Stylasterina (Coelentserata: Hydrozoa). Antarctic Research Series 38: 61-164.

Cairns, S.D. 1983b. A generic revision of the Stylasterina (Coelenterata: Hydrozoa). Part 1. Description of the genera. Bull. Mar. Sci. 33 (2): 427-508.

Cairns, S.D. 1983c. Pseudocrypthelia, a new genus of Stylasterine coral (Coelenterata: Hydrozoa) from the Indonesian region. Beaufortia 33(3): 29-35.

Cairns, S.D. 1984. New records of ahermatypic corals (Scleractinia) from the Hawaiian and Line lslands. Occasional Papers of the Bernice P. Bishop Museum $25(10): 1-30$

Cairns, S.D. 1985. Three new species of Stylasteridae (Coelenterata: Hydrozoa). Proc. Biol. Soc. Wash. 98 (3): 728-739.

Cairns, S.D. 1986a. A revision of the Northwest Allantic Stylasteridae. Smithsonian Contributions to Zoology 418: 131 pages.

Cairns, S.D. 1986b. Stylasteridae (Hydrozoa: Hydroida) of the Galapagos Islands. Smithsonian Contributions to Zoalogy 426: 42pp.

Cairns, S.D. 1987a. Conopora adeta, new species from Australia, the first known unattached Stylasterid. Proc. Biol. Soc. Washington 100(1): 141-146.

Cairns, S.D. 1987b. Range extensions of ahermatypic scleractinia it the Gulf of Mexico. Northeast Gulf Science 9: 131-134.

Caims, S.D. 1988. New records of Stylasteridae (Cnidaria: Hydrozos) from Western Australia, including the description of two new species. Rec. West. Aust. Mus. 14(1): $105-119$.

Cairns, S.D. 1989. A revision of the ahermatypic seleractinia of the Philippine Islands and adjacent waters, Part I:
Fungiacyathidae, Micrabaciidae, Turbinoliniiae, Guynidae and Flabellidae. Smithsonian Contriburions to Zoology $\mathrm{N}^{\circ}$ 486

Caims, S.D. 1990. Antarctic Scleractinia, Vol 1, in J.W. Wägela \& J. Sieg, eds., Synopses of the Antarctic Benthos. Koenigstein, Koeltz Scientific Booka.

Caims, S.D. 1991a. A revision of the ahermatypic Scleractinia of the Galapagoa and Cocos Islands. Smithsonian Contributions to Zoology $\mathrm{N}^{\circ} 504$.

Cairns, S.D. 1991b. A generic revision of the Stylasteridae (Coelenterata: Hydrozoa). Part 3: Keys to the genera. Bull. Marine Science 49(1-2): 538-545.

Cairns S.D. 1991c. The Marine Fauna of New Zealand: Stylasteridae (Cnidaria: Hydroida). New Zealand Oceanographic Institute Memoir 98.

Cairns S.D. 1991d. Cyclohelia lamellata, new genus and species of Stylasteridae (Cnidaria: Hydrozoa) from the Bering Sea. Pacific Science 45 (4): 383-388.

Cairns S.D. 1991e. Catalog of the type specimens of stony corals (Milleporidae, Stylasteridae, Scleractinia) in the National Museum of Natural History, Smithsonian Institution. Smithsonian Contributions to Zoology No. 514.

Collins, N.M. and Morris, M.G. 1985. Threatened Swallowrail Butterflies of the World. The IUCN Red Data Book. IUCN, Gland and Cambridge. vii +401 pp.

D'Abrera, B. 1975. Birdwing Bunterflies of the World. Lansdowne Press, Melbourne. 415 pp.

Favre, D.S. 1989. Internotional Trade in Endangered Species: A guide to CITES. Martinus Nijhoff Publishera, Dordrecht/ Boston/London.

IUCN 1990. 1990 IUCN Red List of Threatened Animals. IUCN, Gland, Switzerland and Cambridge, UK.

Johnson, R.1. 1978. Systematics and zoogeograhy of Plogiolo (=Dysnomia = Epioblasma $)$, an almost extinct genus of freshwater mussels (Bivalvia: Unionidae) from middle North America. Bulletin of the Museum of Comparative Zoology 148(6): 239-320.

Johuson, R.I. 1980. Zoogeography of North American Unionacea (Mollusca: Bivalvia) north of the maximum Pleistocene glaciation. Bulletin of the Museum of Comparasive Zoology 149(2): 77-189.

Laborel, J. 1970. Madréporaires et hydrocoralliaires récifaux des cotes brésiliennes, systematique, ecologie, repartition verticale et geographique. Annls. Inst. Oceanogr. Paris 47 (1): 171-229.

Miller, J.S. 1987. Phylogenetic studies in the Papilioninse (Lepidoptera: Papilionidae). Bulletin of the American Museum of National History 186(4):365-512.

Miller, R.R. 1977. IUCN Red Data Baak. Vol. 4 Pisces. IUCN, Morges. 
Nelson, J.S. 1984. Fishes of the World. 2nd edition. J. Wiley and Sons. New York.

Opresko, D.M. 1974. A study of the classification of the Antipatharia with redescription of 11 speciea. Univeraity Microfilms, Ann. Arbor. 1987: 1-194.

Opresko, D.M. and Bayer, F.M. 1991. Rediscovery of the enigmatic coelenterate Dendrobrachia (Octocorallia: Gorgonacea) with description of two new species. Trans. $R$. Sac. S. Aust. 115: 1-19

Parenti, L.R. 1981. A phylogenetic and biogeographic analysis of Cyprinodontiform fishes. Bulletin of the American Museum of Natural History 168(4): 335-557.

Sheppard, C.R.C. and Sheppard, A.L.S. 1991. Corals and coral communities of Arabia. Fauna of Saudi Arabia 12.

The Times Atlas of the World 1990. Comprehensive (eighth) edition. Times Books, London UK.

Turgeon, D.D., Bogan, A.E., Coan, E.V., Emerson, W.K. Lyons, W.G., Pratt, W.L., Roper, C.F.E., Scheltema, A., Thompson, F.G. and Williams, J.D. 1988. Common and scientific names of aquaric inveriebrates fram the United States and Canada: mollusks. American Fisheries Society Special Publication 16. Bethesda, Maryland.

United Nations, 1991. Terminology Bulletin No. 342. United Nations, New York.

Veron, J.E.N. 1985. New scleractinis from Australian coral reefs. Rec. West. Aust. Mus. 12 (1): 147-183.

Veron, J.E.N. 1986. Corals of Australia and the Indo-Pacific. Angus and Robertson, North Ryde (N.S.W.).

Veron, J.E.N. 1990a. Checklist of the hermatypic corals of Vanuatu. Pacific Science 44 (1): $51-70$.

Veron, J.E.N. 1990b. Re-examinstion of the reef corals of Cocos (Keeling) Atoll. Rec. West. Aust. Mus. 14 (4): 553-581.

Veron, J.E.N. 1990c. New scleractinia from Japan and other Indo-West Pacific countries. Galaxeo 9: 95-173.

Veron, J.E.N. and Hodgson, G. 1989. Annotated checklist of the hermalypic corals of the Philippines. Pacific Science 43 (3): 234287.

Veron, J.E.N. and Marsh L.M. 1988. Hermatypic corals of Westem Australia: records and annotated species list. Rec. West. Aust. Mus. Suppl. 29: 1-136.

Veron, J.E.N. and Pichon, M. 1976. Scleractinia of Eastern Australia. Part 1. Families Thamnasteridae, Astrocoeniidse, Pocilloporidae. Aust. Inst. Mar. Sci. Monogr. Series 1., 86pp.

Veron, J.E.N. and Pichon, M. 1980. Scleractinia of Eastern Australia. Part III. Families Agariciidac, Siderastreidae, Fungiidae, Oculinidae, Merulinidae, Mussidac, Pectiniidae, Caryophylliidae, Dendrophylliidae. Aust. Inst. Mar. Sci. Monogr. 4: 443pp.

Veron, J.E.N. and Pichon, M. 1982. Scleractinia of Eastern Australia. Part IV. Family Poritidae. Aust. Inst. Mar. Sci. Monogr. 5: 159pp.
Veron, J.E.N., Pichon, M. and Wijsman-Best, M. 1977. Scleractinia of Eastern Australis. Psrt ll. Families Fsviidae, Trachyphylliidse. Aust. Inst. Mar. Sci. Monogr. 3: 1-233.

Veron, J.E.N. and Wallace, C. 1984. Scleractinia of Eastern Australia. Part V. Family Acroporidac. Aust. Inst. Mar. Sci. Manogr. 6: 485 pp.

WCMC, 1992. Global Biodiversity. Status of the Earth's living Resources. Chapman \& Hall, London, UK.

Wells, J.W. 1972. Notes on Indo-Pacific scleractinian corals, VIII. Scleractinian corals from Easter lsland. Pac. Sci. 26 (2): $183-190$

Wells, J.W. 1982. Notes on Indo-Pacific corals. Part 9. New corala from the Galapagos 1slands. Pac. Sci. 36: 211-219.

Wells, J.W. 1983. Annotated list of the scleractinian corals of the Galapagos Islands. In P.W. Glynn and G.M. Wellington (eds.): Corals and coral reefs of the Galapogos islands, $p$. 211-295. University of California Press, Berkeley.

Wells, J.W. and Lang, J.C. 1973. Appendix: Systematic list of Jamaican shallow-water Scleractinia. Bull. Mar. Sci. 23 (1): 55-58.

Wells, S.M., Pyle, R.M. and Collins, N.M. 1983. The IUCN Invertebrate Red Data Book. IUCN, Cambridge and Gland 632 pp.

Wijnstekers, W. 1992 (3rd edition). The Evolution of CITES. A reference to the Convention on International Trade in Endangered Species of Wild Fauna and Flora. The CITES Secretariat, Lausanne, Switzerland.

Wood, E.M. 1983. Carals of the warld. T.F.H. Publications, Neptune City (N.J.).

Zibrowius, H. 1973. Revision des espèces actuelles de genre Enallapsammia Michellotti, 1871, et description de $D$. marenzelleri, nouvelle espèce bathyale à lange distribution: Océan Indien et Atlantique Central (Madreporaria, Dendrophylliidse). Beaufortia 21, No. 276.

Zibrowius, H. 1974a. Scleractinisres des iles Saine Paul et Amsterdam (sud de l'océan Indien). Tethys 5 (4); 747-777.

Zibrowius, H. 1974b. Révision du genre Javania et considérations générales sur les Flabellidae (Scléractiniaires). Bull. Inst. océanogr. Monaca 71, N. 1429: 48 pp.

Zibrowius, H. 1980. Les Scléractinisires de lo Méditerranée et de l'Atlantique nord-oriental. Memoires de l'Institut Oceanagraphic Manaco N. 11.

Zibrowius H. and Cairna, S.D. 1992. Revision of the northeast Atlantic and Mediterranean Stylasteridae (Cnidaria: Hydrozoa). Mémoires du Muséum National d'Histoire Naturelle, Paris Zoologie. Tome (A) 153.

Zibrowius, H. and Gili, J.M. 1990. Deep-water Scleractinis (Cnidaria: Anthozoa) from Namibia, South Africa and Walvis Ridge, Southeastern Atlantic. Scient. Mar. 54(1): $19-46$.

Zibrowius, H. and Grieshaber, A. 1977. Scléractinaires de l'Adriatique. Tethys 4: 375-384. 


\section{Class: OSTEICHTHYES}

\section{Order CERATODIFORMES}

Family CERATODIDAE

Neoceratodus forsteri (Krefft 1870)

Australian Lungfish, Queensland Lungfish

Australia: Queensland

\section{Order COELACANTHIFORMES}

Family LATIMERIIDAE

Latimeria chalumnae Smith 1939

Coelacanth, Gombessa

Comoros; South Africa (ex)

\section{Order ACIPENSERIFORMES}

Family ACIPENSERIDAE

Acipenser brevirostrum LeSueur 1818

Shortnose Sturgeon

Canada $[15,21,40,59,69,90]$; USA $[9,55,70,92,116]$

Acipenser oxyrhynchus Mitchell 1814

Atlantic Sturgeon, Common Sturgeon

Albania; Algeria; Belgium (ex?) [85]; Bulgaria (ex?) [10]; Czechoslovakia (ex) [32,33]; Denmark (ex) [63]; Finland (ex?); France [52,101]; Germany: West (ex) [8,23,106]; Greece [26]; Iceland (ex?); Ireland (ex?) [115]; Italy [1,24]; Morocco; Netherlands (ex?) [79]; Norway (ex?) [84]; Poland (ex?) [29,43]; Portugal [2,3]; Romania [109]; Russia [7]; Spain (ex?) [44,47]; Sweden (ex?) [19]; ?Switzerland; Turkey [54,56]; Ukraine [7]; United Kingdom [65,113]; Yugoslavia (ex?)

\section{Family POLYODONTIDAE}

Polyodon spathula (Walbaum in Artedi 1792)

II V 5 Spoonbill Cat, Duckbill Cat, Spadefish

Canada (ex); USA 


\section{Order OSTEOGLOSSIFORMES}

Family OSTEOGLOSSIDAE

Arapaima gigas (Cuvier 1817)

II $\quad \mathrm{K} \quad 30,36,49,75$

Arapaima, Pirarucu

Brazil $[4,12,96]$; Guyana; Peru [117]

Scleropages formosus (Müller \& Schlegel 1844)

Asian Arowana, Asian Bonytongue

\section{Appendix I populations \\ I K \\ $6,16,27,49,50,53,67,89$}

Cambodia [20]; Lao P.D.R.; Malaysia: Peninsular Malaysia; ?Myanmar; Philippines; Singapore; Thailand (ex?) [102]; Vietnam

Appendix II populations (CITES export quota) Il(eq) K

Indonesia: Kalimantan, Sumatra

\section{Order CYPRINIFORMES}

Family CYPRINIDAE

Caecobarbus geertsi Boulenger 1921

II $\quad$ E 28

African Blind Barb Fish

Zaire: Bas-Zaïre

Probarbus jullieni Sauvage 1880

Ikan Temoleh, Pla Eesok

I $\quad \mathrm{K} \quad 6,49,75,81,105$

Cambodia [20,53]; Lao D.P.R.; Malaysia [48]; Thailand [94]; Vietnam

Family CATOSTOMIDAE

Chasmistes cujus Cope 1883

$1 \quad E$

E $\quad 18,49,76,88,95$

Cui-ui

USA: Nevada

\section{Order SILURIFORMES}

Family SCHILBEIDAE ( = PANGASIIDAE)

Pangasianodon gigas Chevey 1930

I V

$6,49,75,78,81,82,86$

\section{Giant Catfish, Pla Buk}

Cambodia [20,53]; China: Yunnan [41]; Lao D.P.R.; Myanmar; Thailand [94, 102]; Vietnam 
CITES RL Ref

\section{Order PERCIFORMES}

Family SCIAENIDAE

Cynoscion macdonaldi Gilbert 1890

I

E

$49,108,110$

(Gulf of California)

Mexico 


\section{REFERENCES - FISH}

1. Alesio, G. and Gandolfi, G. 1983. Censimiento e distribuzione attuale delle specie ittiche nel bacino del fiume Po. Instituto di Ricerca Sulle Acque Quademi 67: $1-92$.

2. Almaça, C. 1988. A lampreia e o esturjâo na Bacia do Douro. Actaa 10 Congresso Internacional sobre o Rio Douro, Vila Nova de Gaiz.

3. Almaça, C. 1988. On the sturgeon, Aclpenser sturio Linnaeus, 1758, in the Portuguese rivero and sea. Folia Zoologica, Bratislava 37(2): 183-191.

4. Anon. 1981. Conservation of nature and natural resources in the Brazilian Amazon. CVRD-reviata, Vol.2, Special Ed. Pp: 37-45.

5. Anon. 1992. Inclusion of Polyodon spathula in Appendix II. Amendments to Appendices I and II of the Convention. Pisces (1): 4-13. Convention on International Trade in Endangered Species of Wild Fauns and Flors, Eighth Meeting of the Conference of the Parties, Kyoto (Japan), March 1992.

6. Bain, J.R. and Humphrey, S.R. 1982. A profile of the endangered species of Thailand. Vol.1. Through birds. Repor No.4. Office of Ecological Services, Florida State Museum, University of Florida, Gainesville, Florida. $367 \mathrm{pp}$.

7. Bannikov, A.G. and Sokolov, V.I. (Eds) 1984. The Red Data Book of the USSR. Rare and Threatened Species of Animals and Plants. Lesnaya Promiishlyennost Press, Moscow. (In Russian.)

8. Blab, J., Nowak, E., Kreft, E., Lelek, A. and Tesch, F.-W. 1977. Rote Liste der Fische (Pisces) und Rundmäuler (Cyclostomata). 2. Fassungi. Stand: 15.3 .1977

9. Blodget, B.G. and Cardoza, J.E. 1983. Nongame wildlife for special consideration in Massachusetts. Fauna of Massachuserts 5: 1-10.

10. Botev, S.B. and Peshev, T. (Eds). (undated). The Red Data Book of Bulgaria. Vol.2 Animals.

11. Böıger, B., Jens, G., Keiz. G., Lelek, A., Mau, G., Müller, D. and Riedel, D. 1979. Gutachten zur der Aufnahme von Fischen in die Artenliste der Bundersartenschutzverordnung. Arbeiten des Deutschen Fischerei-Verbandes 28: 1-109.

12. Britski, H.A. and de Figueiredo, J.L. 1972. Peixes brasileiros que necessitam de protecâo. In: Espécies da fauna brasileira Ameą̧adas de Extinçâo. Academia Brasileira de Ciências, Rio de Janeiro, Gb.

13. Burton, R., Kennedy, M. and Fry, 1. 1986. The threatened vertebrates. In: Kennedy, M. and Ross, B. (Eds), A Threatened Species Conservation Strategy for Australia. - Palicies for the Fuure. Pp. 8-12.

14. Campbell, K.S.W. 1981. Lungfishes - alive and extinct Field Museum Naural History Bulletin 52(8): 3-5

15. Campbell, R.R. 1984. Rare and endangered fishes of Canada: The Committee on the status of endangered wildlife in Canada (COSEWIC) Fish and Marine Mammals Subcommittee. Canadian Field-Naturalist 98(1): 71-74.

16. Chiam, E. 1987. Swimming gold from Malaysia. Tropical Fish Hobbyist June 1987.
17. Côrtes de Lacerda, M.T. 1987. Comentarios sobre as espécies de Cynolebias incluidar na lista dos peixes ameaçadoa de extinçao. Revista de Aquariofilia 3: 34-36.

18. Cui-ui Recovery Tesm 1983. Revised Cui-ui Recovery Plan November 22, 1983. U.S. Fish and Wildlife Service Portland, Oregon.

19. Curry-Lindahl, K. 1985. Vara fiskar. Havs-och Sötvattensfiskar i Norden och övriga Europa. P.A. Norstedt and Sönera Förlag. Stockholm.

20. D'Aubenton, F. 1963. Rapport aur le fonctionnement d'un barrage mobile aur le Tonlé-Sap. République Française. Ministère dę Affairea Errangères. Mission Francaise d'Aide Economique et Technique au Cambodge. Muséum National d'Histoire Naturelle.

21. Dadswell, M.J. 1984. Status of the shortnose sturgeon, Acipenser brevirostrum, in Canads. Canadian Field Naturalist 98(1): 75-79.

22. Dadswell, M.J., Tauber, B.D.; Squires, T.S.; Marchette, D. and Buckley, J. 1984. Synopsis of biological data on shortnose sturgeon, Acipenser brevirostrum Le Suer 1818. NOAA Technical Report NMFS 14. FAO Fisheries Synopsis 140: 1-45.

23. Dehus, P. 1982. Rote Liste der Süsswasserfische Schleswig-Holsteins. 1. Fassung. In: Rote Listen der Pllanzen und Tiere Schleswig-Holsteins. Schrifienreihe des Landesamtes für Naturschutz und Landschafispflege Schleswig-Holstein, Kiel. Pp. 95-97.

24. Delmastro, G.B. 1982. Guida ai pesci del Bacino del Po - e delle acque dolci d'lialia. Museo Civico di Storia Naturale di Carmagnola. CLESAV, Milano.

25. Duncker, G., Ehrenbaum, E., Kyle, H.M., Mohr, E.W. and Schnakenheck, W. 1929. Die fische der Nond- und Ostsee. Akademische Verlags GmbH, Leipzig. (In German.)

26. Economidis, P.S. 1973. Catalogue des Poissons de la Grèce. Hellenic Oceanology and Limnology 11:421-598 (in French).

27. Ensoll, B., Furtado, J.I. and Scott, D.B.C. (undated). Notes on Scleropages formosus (Müller and Schlegel) in Malaya.

28. Evans, D. 1985. Caecoharbus geerzi. In: Dollinger, P. (Ed.), CITES Identification Manual. Vol.3. Reptilia, Amphihia, Pisces. Secretariat of the Convention. Lausanne, Switzerland

29. Ferens, B. 1965. Animal species under protection in Poland (Ochrona gatunkowa zwierzat w Polsce). Translated from Polish. Sci. Pub. Forg. Coop. Center Central Instl Sci. Tech. and Economic Information.

30. Fink, W.L. and Fink, S.V. 1979. Centrsl Amazonia and its fishes. Comparative Biochemistry and Physiology 62A: 13-29.

31. Flasar, 1. and Flasarova, M. 1975. Die Wirbeltierfauna Nordwestböhmens (severozapadni Cechy) Die bisherigen Ergebnisse ihrer Erforschung. Zoalogische Abhandlungen Staalliches Museum filr Tierkuinde, Dresden 33: 1-150.

32. Flasar, 1. and Flasarova, M. 1981. O Rybach Reky Ohre. Krajské Museum Teplice-Povodi Ohre Chomutov. Teplice. 
33. Flasar, 1. and Flasarova, M. 1985. Bibliographie ūber die Fische des Flusses Ohre (Eger, CSSR) und seiner Zuflüsse (Cyclostomata et Osteichthyes). Zoologische Abhandlungen Staatliches Museum fur Tierkunde, Dresden 41(6): 77-91.

34. Fricke, H. and Schamer, J. 1987. Im Reich der lebenden Fossilien. Geo 10: 15-34.

35. Fry, 1. and Kennedy, M. 1986. Correlating habitats with high priority threatened species. Chapter VI. In: $M$. Kennedy and R. Burton (Eds). A Threatened Species Conservation Strategy for Australia - Policies for the future. Ecofund Australia. Pp. 39-42.

36. Géry, J. 1969. The freshwater fishes of South America. In: E.J. Fittkau, J. Illies, H. Klinge, G.H. Schwahe H. Sioli (Eds), Biogeography and Ecology in South America. Vol.2. Junk, The Hague. Pp. 828-848.

37. Gilbert, C.R. 1978. Fishes. In: P.C.H. Pritchard (Series Ed.), Rare and endargered biota of Florida, Vol.4. University Presses of Florida

38. Gonçalves da Cruz, C.A. 1983. Uma nova espécie de Cynolebias do Estado do Espiritu Santo, Brazil, (Pisces, Cyprinodontidae). Papéis Avulsos de Zoologia, S/a Paulo 35(6): 73-77.

39. Gonçalves de Cruz, C.A. and Peixoto, O.L. 1976. Notas sobre Cynolebias constancioe Myers, 1942 (Osteichthyes, Cyprinodontidae, Rivulinae). Revista Brasileira de Biologia 36(2): 377-379.

40. Gorham, S.W. and McAllister, D.E. 1974. The shortnose sturgeon, Acipenser brevirosirum in the Saint John River, New Brunswick, Canada, a rare and possibly endangered species. Syllogeus 5: 1-18.

41. Gressitt, J.L. 1970. Biogeography of Laos. Pacific Insects Monography 24: 573-626.

42. Gruchy, C.G.and Parker, B. 1980. Shortnose sturgeon, Acipenser brevirostrum. In: Lee, D.S., Gilbert, C.R., Hocutt, C.H., Jenkins, R.E., McAllister, D.E. and Stauffer, J.R. (Eds), Atlas of North American Freshwater Fishes. North Carolina State Museum of Natural History. Raleigh.

43. Gtowacinski, Z., Bieniek, M., Dyduch, A.; Gertychowa, R.; Jakubiec, Z; Kosior, A. and Zemanek, M. 1980. Situation of all vertebrates and selected invertebrates in Poland - List of species, their occurrence, endangerment and status of protection. Warszawa-Krahow. Polska Akademia Nauk.

44. Hernando, J.A. 1975. Notas sobre distribucion de los peces fluviales en el Sur-oeste de Espa/a. Do/ana, Acia Vertebrata 2(2): 263-264.

45. Hoese, H.D. and Moore, R.H. 1977. Fishes of the Gulf of Mexico - Texas, Lowisana and Adjacent Waters. Texas A \& M University Press, College Station.

46. Hubber, J.H., Seegers, L. and Wildekamp, R.H. 1986. Liste Actualisée des Cyprinodontides. $44 \mathrm{pp}$.

47. ICONA (Ed.) 1986. Lista Roja de los Vertebrados de Esparia. Publicaciones del Ministerio de Agricultura, Pesca y Alimentacion, Madrid. (In Spanish.)

48. Ismail, M.Z. 1984. Checklist of fishes of Taman Negars. Malayan Naturalist 37(3): 21-26.

49. IUCN 1990. The IUCN Red List of Threatened Animals. IUCN Gland, Switzerland and Cambridge, UK. Pp. 85-105.
50. Joseph, J., Evans, D. and Broad, S. 1986. International Trade in Bonytongues. TRAFFIC Bullerin 7(5): 73-76.

51. Kemp, A. 1982. The embryological development of the Queenaland lungfish Neoceratodus forsteri (Krefft). Memoirs of the Queensland Museum 20(3): 553-597.

52. Kiener, A. (undated). Espèces en voie de disparition ou menacées dana le Midi Méditerranéen.

53. Kottelat, M. 1985. Fresh-water fishes of Kampuchea. A provisory annotated check-list. Hydrobiologia $121: 249-279$.

54. Kuru, M. 1980. Tilrkiye Tattisu Baliklari Katalogu. Turkaye Faunasi 12(1): 1. (In Turkish.)

55. Kynard, B., Buckley, 1. and Gabriel, W. 1982. Shortnose sturgeon biology below Holyoke Dam. Massachussetts Cooperative Fisherieg Research Unit, University of Massachussetts, Amherat.

56. Ladiges, V.W. 1964. Süsswasserfische der Türkei. Mitteilungen aus den Hamburgischen Zoologischen Museum und Institut 61: 203-220. (In German.)

57. Lazara, K. 1981. Developments, problems and synonymy in the genus Cynolebias. Journal of the American Killifish Associotion 14(3): 67-72.

58. Lazara, K. 1984. (Ed.). Killifish Master Index. 3rd Edition. American Killifish Associarion. Pp. 295.

59. Leim, A. H. and Day, L. R. 1959. Records of uncommon and unusual fishes from eastern Canadian waters, 1950-1958. Joumal of the Fisheries Research Baard of Canado 16: $503-514$

60. Lelek, A. 1980. Threatened freshwater fishes of Europe Council of Europe, Strasbourg.

61. Lelek, A. 1987. Threatened fishes of Europe. In: Council of Europe, The freshwater fishes of Europe. $343 \mathrm{pp}$.

62. Locket, N.A. 1980. Some advances in coelacanth biology. Proceedings of the Royal Society of Lendon B. 208: 265-307.

63. Lojtnant, B. and Gregersen, J. 1986. Truede Planter og dyr $i$ Danmark. (Threatened plants and animals in Denmark - a collection of red lists). Fredningsstyrelsen and Lanbrugsministeriets Vildt for Valtning. Pp. 34-35. (In Daruish.)

64. Lythgoes, J. and Lythgoes, G. 197!. Fishes of the Seo. Blandford Press, London.

65. Maitland, P.S. 1985. Criteria for the selection of important sites for freshwater fish in the British Isles. Biological Conservarion 31(1985): 335-353.

66. Maitland, P.S. 1986. Conservarion of threatened freshwater fish in Europe. Council of Europe, Strasbourg, 18-20 November 1986.

67. Matsumura, S. and Milliken, T. 1984. The Japanese trade in bony tongue and CrTES-listed fish. Traffic Bullerin $6(3-4): 42-50$.

68. McAltister, D.E. 1971. Old fourlegs - A "living fossil". National Museums of Canada. Odyssey Series 1: 1-25.

69. McAllister, D.E., Parker, B.J. and McKee, P.M. 1985. Rare, endangered and extinct fishes in Canada. Syllogeus 54: 1-192. 
70. Menhinick, E.F., Burton, T.M. and Bailey, J.R. 1974 An annotated checklist of the freshwater fishes of North Carolina. The Joumal of the Elisha Mitchell Scientific Society $90(1): 2450$.

71. Merrick, J.R. 1984. Australian Freshwater Fishes Biology and Management. Griffin Press Ltd, Austrelia.

72. Michaelis, F.B. 1985. Threatened fish. A report on the threatened fish of inland waters in Australia. Australisn National Parks and Wildlife Service. Report Series 3: $1-45$.

73. Michaelis, F.B. 1986 . Conservation of Australian aquatic fauna. In De Deckker, P. and Williams, W.D. (Eds), Limnology in Australia. CSIRO, Melboume and W. Junk, Dordrecht. Pp. 599-613.

74. Michaelis, F.B. 1987. Protection of Australian Inland Fighe日. In: Harris, J.H. (Ed.), Proceedings of the Conference on Australian Threatened Fishes, Melbourne 15-16 August 1985. Australian Society for Fish Biology. Division of Fisheriea, Department of Agriculture, New South Wales. Sydney. Pp. 19-24.

75. Miller, R.R. 1977. IUCN Red Data Book. Vol. 4 Pisces. IUCN, Morgeв.

76. Moyle, P.B. 1974. Inland Fishes of Califormia. University of California Press, Berkeley, Los Angeles, London.

77. Murawaki, S.A. and Pacheco, A.L. 1977. Biological and fisheries data on Atlantic sturgeon, Acipenser axyrhynchus (Mitchell). Technical Series Repon 10: 1-68.

78. Nelson, J.S. 1984. Fishes of the World. 2nd edition. J. Wiley and Sons. New York.

79. Nijsen, H. and de Groot, S.J. 1987. De Vissen van Nederland. Utrecht, KNNV. Pp. 1-223. (In Dutch.)

80. NOAA (National Ocesnic and Atmospheric Administration) 1987. Status review of shornose sturgeon (Acipenser brevirostrum) Le Sueur 1818). Liated under the Endangered Species Act of 1973. (Unpublished).

81. Pantulu, V.R. 1972. Mekong Fishery Programme - Its background and Rationale. - U.N. Economic Commission for Asia and the Far East. Bangkok, Thailand.

82. Pantulu, V.R. 1973. Fishery problems and opportunities in the Mekong. In: W.C. Ackermann, G.F. White and E.B. Worthington (Eds). Man-made lakes: Their problems and Environmental Effects. Geophysical Monograph Series 17: $672-682$

83. Parenti, L.R. 1981. A phylogenetic and biogeographic analysis of Cyprinodontiform fishes. Bullerin of the American Museum of Natural History 168(4): 335-557.

84. Pethon, P. 1985. Aschehougs siore fiskebok. Oslo.

85. Poll, M. 1947. Faune de Belgique - Poissons marins. Musèe Royal d'Histoire Naturelle de Belgique. Bruxelles.

86. Pookaswan, T. 1969. Pangasianodon gigas Chevey. Inland Fishery Division, Dept of Fisheries, Bangkok, Thailand 7: 1-12

87. Rühmer, K. 1954. Fish and other marine animals. Capture and utilizarion.

88. Scoppettone, G.G., Coleman, M. and Wedemeyer, G.A 1986. Life history and status of the endangered Cui-ui of Pyramid Lake, Nevada. Fish and Wildle Research 1: $1-23$
89. Scou, D.B.J. and Fuller, J.D. 1976. The reproductive biology of Scleropages formosus (Müller and Schlegel) (Osteoglossomorpha, Osteoglossidae) in Malaya, and the morphology of its pituitary gland. Joumal of Fish Biology 8: $45-53$.

90. Scott, W.B. 1967. Freshwater Fishes of Eastem Canada. 2nd Edition. University of Toronto Preas, Canada.

91. Scott, W.B. and Crosaman, E.J. 1973. Freshwater fishea of Canada. Fisheries Research Boord of Canada Bulletin 184: $1-966$

92. Shiffer, C., Walke, T. and U1sh, S. 1985. Endangered and threatened species of Pennsylvaria.

93. Shortnose Sturgeon Recovery Plan 1982. National Marine Fisheries Service in cooperation with the Recovery Team.

94. Sidthimunka, A. 1970. A report on the fiaheries aurveys of the Mekong River in the vicinity of the Pa Mong Dam site. Department of Fisheries, Thailand Technical Paper 8: 1-75 (quoted by Pantulu, 1972).

95. Sigler, W.F., Vigg, S. and Bres, M. 1985. Life history of the Cui-ui Chasmistes cujus Cope, in Pyramid Lake, Nevada: A review. The Great Basin Naturalist 45(4): $571-603$.

96. Smith, N.J.H. 1985. The impact of cultural and ecological change on Amazonian fisheries. Biological Conservarion 32: 355-373.

97. Smith, T.I.J. 1985. The fishery, biology, and managment of Atlantic sturgeon, Acipenser arymynchus, in North America. Environmental Biology of Fishes 14(1): 61-72.

98. Smith, T.I.J. and Dingley, E.K. 1984. Review of biology and culture of Atlantic Sturgeon (Acipenser axyrhynchus) and Shortnose Sturgeon (A. brevirostrum). Journal of the World Mariculture Society 15: 210-218.

99. Smith, T.I.J., Dingley, E.K. and Marchette, D.E. 1980. Induced spawning and culture of Atlantic sturgeon. Progressive Fish-Culturist 42(3): 147-151.

100. Smith-Vaniz, W.F. 1968. Freshwaier Fishes of Alabama. Auburn Univergity Agriculture Experimental Station, Auburn, Alabama.

101. Spillmann, C.J. 1961. Faune de France. 65 Poissons d'eau douce. Fédération Française des Sociétés de Sciences Naturelles, Paris. Pp. 23-25.

102. Suvatti, C. and Menasveta, D. 1968. Threatened apeciea of Thailand's aquatic fauna and preservation problems. In: Talbot, L.M. and Talbot, M.H. (Eds), Conservarion in tropical South east Asia. IUCN Publication. New Series 10.

103. Swift, C.C. 1986. Zoogeogrephy of the Freshwater Fishes of the Southeastern United States - Savannah River to Lake Pontchartrain. In: Hocult, C.H. and Wiley, E.O. (Eds). The Zoogeogrophy of North American Freshwater Fishes. pp. 213-265.

104. Tardif, A. 1984. Rapport sur la gituation de l'esturgeon noir au Québec. (Acipenser oxyrhynchus). Faune et flore a protéger au Québec. Association des Biologistes du Québec Publicarion 6: 1-27.

105. Taylor, E.C. 1983. Discovering and identifying two cyprinids. Tropical Fish Habbyist 31(10): 70-73.

106. Terofal, F. 1977. Das Artenspektrum der Fische Bayerns in den letzten 50 Jahren. Ber. ANL 1: 9-22. 
107. Thompson, J.M. 1974. Fish of the Ocean and Shore Australian Naturalist Library. Collins, Sydney.

108. U.S. Fish and Wildlife Service 1979. Totoaba; listing as an endangered species: Final Regulation. Federal Register 44(99): 29478-29480.

109. Vasiliu, G.D. and Sova, C. 1968 . Fauna Vertebratica Romaniae (Index). Muzeul Judetean Bacau. Pp. 10-73.

110. Villamar, A. 1980. Tolaba, un nuevo género de la familia Sciaenidae en el Golfo de California, México (Pisces: Teleostei). Anales de la Escuela Nacional de Ciencias Biologicas 23: 129-133.

111. Vladykov, V. D. and Greeley, J. R. 1963. Order Acipenseroidei. In: Fisheg of the Western North Allantic. Pan III. Memoirs of the Sears Foundation for Marine Research 1: 24-60.

112. Wolden, H.T. 1964. Familiar Freshwater Fishes of America. Harper and Row, New York.
113. Wheeler, A. 1973. Leonard Jenyna's Nates on Cambridgeshire Fishes. Cambridgeshire and Isle of Ely Naturalists' Trust Annual Report 1973: 19-22.

114. Williams, J.E., Johnson, J.E., Hendrickson, D.A., Contreras-Balderas, S., Williams, J.D., Navarro-Mendoza, M., McAllister, D.E. and Deacon, J.E. 1989. Fishes of North America endangered, threatened, or of specia] concern: 1989. Fisheries, 14(6): 2-21.

115. Wilson, J.P.F. and Flower, R.J. 1980. A large sturgeon A. sturio from Ardglas8, Co. Down. Irish Naturalists' Joumal 20(1): 1-43.

116. Wood, D.A. 1983. Endangered and potentially endangered fauna and flora in Florida. Florida Game and Freshwater Fish Commiasion. Official lists.

117. Wosnitza-Mendo, C. 1984. The growth of Arapaima gigas (Cuvier) after stocking in a Peruvian lake. Archiv fur Fischerei Wissenschaft 35(1-2): 1-5. 



\section{Phylum: ARTHROPODA}

\section{Class: INSECTA}

\section{Order LEPIDOPTERA}

Family PAPILIONIDAE

Note, for the birdwing butterflies, genera Ornithoptera, Trogonoptera and Troides, specific and subspecific status follows D'Abrera [70]. Subsequent revisions have been referenced but only totally new taxa have been added. Subspecies are listed where possible as they may occasionally be listed as full species.

$\begin{aligned} & \text { Bhutanitis lidderdalii Atkinson 1873 } \\ & \text { Bhutan Glory } \\ & \text { Bhutan; China: Sichuan, Yunnan; India [157,253]; Myanmar; Thailand }\end{aligned}$
$\begin{aligned} & \text { Bhutanitis ludlowi Gabriel 1942 } \\ & \begin{array}{l}\text { Ludlow's Bhutan Swallowtail } \\ \text { Bhutan: Trashiyangsi Valley }\end{array}\end{aligned}$
$\begin{aligned} & \text { Bhutanitis mansfieldi (Riley 1939) } \\ & \begin{array}{l}\text { Mansfield's Three-tailed Swallowtail } \\ \text { China: Sichuan, Yunnan }\end{array}\end{aligned}$
$\begin{aligned} & \text { Bhutanitis thaidina (Blanchard 1871) } \\ & \text { Chinese Three-tailed Swallowtail } \\ & \text { China [195] }\end{aligned}$

Ornithoptera akakeae Kobayashi and Koiwaya $1978 \quad 11 \quad-\quad 63,110,143$

(believed to be a natural hybrid between $O$. priamus poseidon and $O$. rothschildi) Indonesia: Arfak Mountains in Irian Jaya

Ornithoptera alexandrae (Rothschild 1907) I I I $\quad$ E $\quad 20,63,69,110,169,177,190,191$ Queen Alexandra's Birdwing

Papua New Guinea: Popondetta in Northern Province of New Guinea

Ornithoptera allottei (Rothschild 1914) $11 \quad$ - $\quad 63,70$

(believed to be a natural hybrid between $O$. urvilliamus and $O$. victoriae regis)

Abbe Allotte's Birdwing

Solomon Islands: Malaita [204]; Papua New Guinea: Bougainville

Ornithoptera caelestis (Rothschild 1898) 
Ornithoptera chimaera (Rothschild 1904)

II

Jd

$20,63,110,169,189,190$

(subspecies: charybdis, chimaera, flavidior)

Chimaera Birdwing

Indonesia: Irian Jaya; Papua New Guinea: New Guinea

Ornithoptera croesus Wallace 1859

II

V

$20,63,70,110,255$

(subspecies: croesus, lydius)

Indonesia: Moluccas

Ornithoptera goliath Oberthür 1888

(subspecies: goliath, procus)

Goliath Birdwing

Indonesia: Irian Jaya, Moluccas; Papua New Guinea: New Guinea, Goodenough Island

Ornithoptera meridionalis (Rothschild 1897)

II V

$20,63,76,102,110,169,189,192$, 193

(subspecies: meridionalis, tarunggarensis)

(subspecies tarunggarensis may belong to $O$. paradisea)

Indonesia: Irian Jaya; Papua New Guinea: New Guinea

Ornithoptera paradisea Staudinger 1893 II Id

(subspecies: arfakensis, borchi, chrysanthemum, flavescens, paradisea)

Paradise Birdwing, Tailed Birdwing, Butterfly of Paradise

Indonesia: Irian Jaya; Papua New Guinea: New Guinea

Ornithoptera priamus (Linnaeus 1758)

11

$20,62,63,65,103,110,127,143$ $166,187,203$

(subspecies: admiralitatus, arruana, boisduvali, bornemanni, euphorion, gebeensis, hecuba, miokensis, poseidon, priamus)

Priam's Birdwing, Common Birdwing, Common Green Birdwing, New Guinea Birdwing

Australia: Queensland; Indonesia; Papua New Guinea; Solomon 1slands [204,205]

Ornithoptera richmondia (Gray 1852)

II

$20,63,65,103,110,166$

Richmond Birdwing

Australia: Queensland, New South Wales

Ornithoptera rothschildi Kenrick 1911

Rothschild's Birdwing

$11 \quad$ ld $20,63,110,143,189$

Indonesia: Arfak Mountains in Irian Jaya

Ornithoptera tithonus de Haan 1840

(subspecies: misoolana, rithonus, waigeuensis)

11

K

$20,63,78,110,189$

Indonesia: Irian Jaya

Ornithoptera urvillianus (Guérin-Méneville 1829)

II

$20,63,103,110$

D'Urville's Birdwing

Papua New Guinea: Bismarck Archipelago, Bougainville; Solomon Islands [156,205] 
Table 1: Conservation status of subspecific taxa of Ornithoptera priamus (after N.M. Collins [5])

1. admiralitatis

2. arruana

3. boisduvali

4. bornemanni

5. euphorion

6. gebeensis

7. hecuba

8. miokensis

9. poseidon

10. priamus
Papua New Guinea (Admiralty Islands)

Indonesia (Aru, off Halmahera)

Papua New Guinea (Woodlark Islands)

Papua New Guinea (New Britain)

Australia (N. Queensland)

Indonesia (Gebe Island)

Indonesia (Kai, Walim, Laut)

Papua New Guinea (Duke of York Island)

Papua New Guinea and 1rian Jaya (Waigeo mainland

and adjacent islands), Australia (Cape York)

Indonesia (Ceram, Ambon) nt
$R$ ?
$\mathrm{R}$
$\mathrm{nt}$
$\mathrm{Id}$
$\mathrm{K}$
$\mathrm{R}$ ?
$\mathrm{E}$

$\mathrm{nt}$
$\mathrm{nt}$

Various forms of $O$. p. poseidon are occasionally cited or sold as separate subspecies. These include:

archideus from Indonesia: Waigeo

cronius from SW coast of Irian Jaya, Indonesia

demophanes from Papua New Guinea: Fergusson, Trobriands

pronomus or macalpinei from The lron Range, Cape York, Australia

teucrus from Indonesia: Biak, Schouten

The priamus group has been the subject of much revision and taxonomic status is disputed in several cases. Certain taxa treated as full species in this list may therefore be treated by other authors as subspecies. The taxa where this is most likely are $O$. caelestis, $O$. richmondia and $O$. urvillianus but others may also be involved.

\section{Ornithoptera victoriae Gray $1856 \quad$ II $\quad-\quad 20,50,63,70,110,169$}

(subspecies: archeri, epiphanes, isabellae, reginae, regis, rubianus, victoriae)

Queen Victoria's Birdwing

Papua New Guinea: Bougainville; Solomon Islands [156,204,205]

Papilio chikae Igarashi 1965

I E

$63,72,104,126,141,177,255$

Luzon Peacock Swallowtail

Philippines: Luzon

Papilio homerus Fabricius 1793

Homerus Swallowtail

Jamaica

Papilic hospiton Guenée 1839

Corsican Swallowtail

France: Corsica [38]; Italy: Sardinia [216]

Parnassius apollo (Linnaeus 1758)

Apollo

II

R

E

$12,63,64,85,86,114,120,177$

Albania; Andorra; Armenia; Austria [94]; ?Azerbaijan; Bulgaria; China: Xinjiang Uygur; Czechoslovakia [51,28]; Finland [165,252]; France [27]; Georgia; Germany: East (ex), West [8,30,146]; Greece; ?Hungary; Italy: including Sicily [35]; Iraq; Iran; Kazakhstan; Kyrgyzstan; Latvia (ex); Liechtenstein [29]; Lithuania (ex); Mongolia; ?Netherlands; Norway; Poland [73,74,75,183, 184]; Romania [206]; Russian Federation; Spain [95]; Sweden [133,132]; Switzerland [49]; Syria; Turkey; Ukraine; Yugoslavia 
Teinopalpus aureus Mell 1923

Golden Kaiser-I-Hind

China: Guangdong [164]; ?Viet Nam

Teinopalpus imperialis Hope 1843

Kaiser-I-Hind, Kaiserihind

Bhutan; China: Hubei, Sichuan [164]; India [253]; Myanmar; Nepal $[229,230]$

Trogonoptera brookiana (Wallace 1856)
K $\quad 63,72,103,177$

II

(subspecies: albescens, brookiana, haugumei, natunensis, trogon)

Rajah Brooke's Birdwing

Brunei; Indonesia; Malaysia

\section{Trogonoptera trojana (Honrath 1886)}

II

$20,63,70,72,109,166,255$

Philippines: Balabac, Palawan

Troides aeacus (C. and R. Felder 1860)

II

$20,62,63,70,72,111,166$

(subspecies: aeacus, kaguya, thomsoni)

(subspecies kaguya is considered Endangered by Collins and Morris [63])

Golden Birdwing, Small Birdwing

?Bangladesh; Bhutan; Cambodia; China: Sichuan [195]; India [84]; Indonesia: Sumatra; ?Lao P.D.R.; Malaysia: Peninsular Malaysia [66]; Myanmar; Nepal [229,230]; Taiwan [227]; Thailand [34]; Viet Nam [233]

Troides amphrysus (Cramer 1782) II - $\quad 20,63,70,72,166,188,255$

(subspecies: amphrysus, andrewei, flavicollis, niasicus, ruficollis, vistara)

Golden Birdwing, Malay Birdwing

Brunei; Indonesia; Malaysia [66,210]; Myanmar: Mergui Archipelago; Singapore; Thailand [34]

Troides andromache (Staudinger 1892) II Id $20,63,70,72,255$

(subspecies: andromache, marapokensis, the latter now regarded as a female form of andromache) Malaysia: Sabah, Sarawak [210]; ?Indonesia: Kalimantan

Troides criton (C. and R. Felder 1860) II - $\quad 20,63,69,70$

(subspecies: celebensis, criton; the former now considered to be unrelated to T. criton. Thought either to be a separate species [144] or a natural hybrid of $T$. haliphron and $T$. helena [103])

Indonesia: Moluccas, Sulawesi (celebensis only)

Troides cuneifer (Oberthūr 1879)

II

$20,63,72,255$

(subspecies: cuneifer, peninsulae, sumatranus)

Golden Birdwing

Indonesja: Java, Sumatra; Malaysia: Peninsular Malaysia; Thailand

Troides darsius (Gray 1852)

II -

$20,63,70,72,84,282$

Sri Lanka

Troides dohertyi (Rippon 1893)

II V

$20,63,103,110,255$

Talaud Black Birdwing

Indonesia: Talaud Islands

Troides haliphron (Boisduval 1836)

II

$20,63,70,72,127,255$

(subspecies: ariadne, ikarus, iris, haliphron, naias, pallens, pistor, socrates, staudingeri)

Indonesia: Sulawesi and southem islands) 
(subspecies: antileuca, cerberus, ferrari, helena, heliconoides, hephaestus, isara, maurus, mopa, moschylus, neoris, nereides, nereis, orientis, propinquus, sagittatus, spilotia, typhaon)

Common Birdwing, Black and Gold Birdwing

Bangladesh; ?Bhutan; Brunei; ?Cambodia; China: Hainan; Hong Kong [139]; India: including Andaman and Nicobar Islands [16]; Indonesia; Lao P.D.R.; Malaysia [66]; Myanmar; Nepal [229,230]; Singapore; Thailand [34]; Viet Nam

Troides hypolitus (Cramer 1775)

II

$63,69,72,166,235,255$

(subspecies: antiope, cellularis, hypolitus, sulaensis)

Indonesia: Moluccas, Sulawesi

Troides magellanus (C. and R. Felder I862)

Il $\quad$ -

$20,62,63,70,72,140,255$

(subspecies: apoensis, magellanus, sonani)

Philippines; Taiwan: Lan Yü Jsland [227]

Troides minos (Cramer 1779)

India

II $\quad$ - $\quad 20,63,70,72$

Troides miranda (Butler 1869)

II

$20,63,70,72,125,255$

(subspecies: miranda, neomiranda)

Brunei; Indonesia: Kalimantan, Sumatra; Malaysia: Sabah, Sarawak [210]

Troides oblongomaculatus (Goeze 1779)

II

$20,26,62,63,69,70,72,255$

(subspecies: bandensis, bouruensis, hanno, oblongomaculatus, papuensis, thestius)

Indonesia; Papua New Guinea [203]

Troides plateni Staudinger 1888

JI

$20,63,70,72,103,109,110,255$

Philippines: Palawan

Troides plato Wallace 1865

IJ

$20,63,69,70,110,255$

Indonesia: Timor

Troides prattorum (Joicey and Talbot 1922)

II

Id

$20,63,69,70$

Buru Opalescent Birdwing

Indonesia: Buru

Troides rhadamantus (Lucas I835)

I]

$20,62,63,70,72,255$

Philippines

Troides riedeli (Kirsch 1885)

II

$20,63,69,70,255$

Indonesia: Tanimbar Islands

Troides vandepolli (Snellen 1890)

(subspecies: honrathiana, vandepolli)

Indonesia: Java, Sumatra 


\section{Class: ARACHNIDA}

\section{Order ARANEAE}

\section{Family THERAPHOSIDAE}

Brachypelma smithi (Pickard-Cambridge 1897) Mexican Red-kneed Tarantula Mexico
I1

K

231,279 
CITES RL Ref

\section{Phylum: ANNELIDA}

\section{Class: HIRUDINEA}

Order ARHYNCHOBDELLAE (=ARHYNCHOBDELLIDA)

Family HIRUDINIDAE

Hirudo medicinalis Linnaeus 1758

II Id

$12,15,64,81,149,154,155,219$, $220,221,222,223,228,277,279$. $280,281,284$

\section{Medicinal Leech}

Albania; Armenia; Austria; ?Azerbaijan; ?Belarus; Belgium [158]; Bulgaria [213,214]; Czechoslovakia [145]; Denmark [17,24,135]; ?Estonia; Finland; France [77]; Georgia; Germany [116]; Greece [250]; Hungary [142,150]; Ireland (ex) [159]; Italy [167,168]; ?Kazakhstan; ?Latvia; Lithuania; Luxembourg [122, 123,124]; Moldova; Netherlands [79]; Norway [178,258]; Poland [134]; Portugal; Romania [68]; Russian Federation; Spain [136]; Sweden [87]; Switzerland; Turkey; Ukraine; United Kingdom [80,82,225]; Yugoslavia [236] 


\section{Phylum: MOLLUSCA}

\section{Class: BIVALVIA}

\section{Order VENEROIDA}

Family TRIDACNIDAE

Hippopus hippopus (Linnaeus 1758)

II Id

$1,36,37,117,173,174,197,211,26$

0,261

Bear Paw Clam, Horse's Hoof Clam, Strawberry Clam

(Indian Ocean: including Bay of Bengal; Indopacific: including South China Sea; Pacific Ocean: including East China Sea; South Pacific: including Coral Sea)

American Samoa (ex?); Australia: Queensland [194], Westerm; Federated States of Micronesia; Fiji (ex?); Guam (ex?); ?India: Andaman and Nicobar Islands [207]; Indonesia; Japan: Bonin Islands (ex?), Kyukyu Islands (ex?); Kiribati: Gilbert Islands; Malaysia; Marshall Islands; Myanmar; New Caledonia; Northern Marianas (ex?); Palau [43]; Philippines [4]; Papua New Guinea; ?Paracel Islands; Samoa (ex?); Singapore; Solomon Islands; Taiwan (ex?); ?Thailand; Tonga (ex?) [160]; Tuvalu; Vanuatu

Hippopus porcellanus Rosewater 1982 II Id $11,173,197,260$

\section{China Clam}

(Indopacific: including Flores Sea, South China Sea)

Indonesia [267]; Palau; Philippines: Sulu Archipelago, Masbate 1sland [4,212]; South China Sea reefs

Tridacna crocea Lamarck 1819

$11 \quad \mathrm{~K}$

$36,101,121,174,211,260,261,268$ 279

Crocus Clam, Saffron-coloured Clam, Boring Clam

(Indopacific: including South China Sea; Pacific Ocean; South Pacific: including Coral Sea)

Australia [88,194]; Guam (ex?); Indonesia [40]; Japan: Ryukyu 1slands; Malaysia; Northem Marianas (ex?); Palau [43,105]; Papua New Guinea [272]; Philippines [4]; Singapore; Solomon Islands; Thailand; ?Tuvalu; ?Vanuatu; Viet Nam

Tridacna derasa (Rōding 1798)

11

\section{Southern Giant Clam}

(Indopacific; Pacific Ocean; South Pacific: including Coral Sea)

[American Samoa]; Australia: Queensland [88,194]; ?Cocos (Keeling) Islands; [Cook Islands]; [Federated States of Micronesia]; Fiji; ?French Polynesia: Tuamotu Archipelago; Guam (ex?); Indonesia: Irian Jaya [217], Taka Bone Rate in Sulawesi [261]; [Marshall Islands]; New Caledonia; Northern Marianas (ex?); Palau [43,105]; Papua New Guinea [272]; Philippines [4]; Solomon Islands; Tonga: Tongatapu [160]; ?Tuvalu; ?Vanuatu

Tridacna gigas (Linnaeus 1758)

II V

$1,36,37,40,67,117,121,171,173$, $174,197,260,261,267,268,279$

\section{Giant Clam}

(Indian Ocean; Indopacific: including South China Sea; Pacific Ocean; South Pacific: including Coral Sea) Australia: Queensland [194], Western; Federated States of Micronesia: Lamotrek Atoll, Wesy Fagu; Fiji (ex?); [Guam (ex?)]; [Hawaiian 1slands]; Indonesia [217]; Japan: Ryukyu Islands (ex?); Kiribati: Gilbert Islands [172]; Malaysia; Marshall Islands; Myanmar; New Caledonia (ex?); Northern Marianas (ex?); Palau [43,105]; Papua New Guinea [272]; Philippines [4]; Solomon Islands; Taiwan (ex?); Thailand; Tuvalu; [USA]; Vanuatu (ex?) 


\section{Small Giant Clam}

(Indian Ocean: including Bay of Bengal, Red Sea, West coast of Africa; Indopacific: including South China Sea; Pacific Ocean: including East China Sea; South Pacific: including Coral Sea, Tasman Sea)

American Samoa; Australia: including Lord Howe 1sland [88, 161,194]; British Indian Ocean Territory: Chagos Archipelago; China; Cook Islands; Egypt; Federated States of Micronesia; Fiji; French Polynesia [218]; Guam; Hong Kong (ex) [261]; India: Andaman and Nicobar Islands [207], Laccadive Islands; Indonesia; Japan; Kenya; Kiribati: Gilbert, Phoenix, Line Islands; Madagascar; Malaysia; Maldives; Marshall Islands; Mauritius; Mozambique; Myanmar; New Caledonia; Northern Marianas; Palau [43,105]; Papua New Guinea [272]; Philippines [4]; Pitcaim Islands: Henderson Island; Samoa; Saudi Arabia [31]; Seychelles; Singapore; Solomon Islands; South Africa; Sri Lanka; Taiwan; Thailand; Tokelau; Tonga [160]; Tuvalu; Vanuatu; Viet Nam; Wake Island

\section{Scaly Clam, Fluted Clam}

(Indian Ocean: including Bay of Bengal, Red Sea, West coast of Africa; Indopacific: including South China Sea; Pacific Ocean; South Pacific: including Coral Sea)

American Samoa; Australia [88,194]; British Indian Ocean Territory: Chagos Archipelago; Egypt; Federated States of Micronesia; Fiji; French Polynesia: Tuamotu Archipelago; [Guam (ex?)]; [Hawaiian 1slands]; India: Andaman and Nicobar Islands [207], Laccadive Islands; Indonesia; Japan (ex?); Kenya; Kiribati: Gilbert Islands [172]; Madagascar; Malaysia; Maldives; Marshall Islands; Mauritius; Mozambique; Myanmar; New Caledonia; Northern Marianas (ex?); Palau [105]; Papua New Guinea [272]; Philippines [4]; Samoa; Saudi Arabia [31]; Seychelles; Singapore; Solomon Islands; South Africa; Sri Lanka; Thailand; Tokelau; Tonga [160]; Tuvalu; [USA]; Vanuatu; Viet Nam

Tridacna tevoroa Lucas, Ledua and Braley 1990 II $\quad 153$ Tevoro Clam

(South Pacific)

Fiji: Lau Islands; Tonga: Ha'apai and Vava'u Groups

\section{Order UNIONOIDA}

\section{Family UNIONIDAE}

The taxonomy of this group, especially the genus here called Epioblasma [55], has had many revisions and the names as listed are not necessarily those now considered taxonomically correct. As many recent synonyms as possible are indexed or listed, including those suggested by Turgeon et al (1988) [256], as standard reference names. Distributional information varies between sources and is also confused by synonomy. Where given (up to a maximum of six states) it should be used as a guide only.

Conradilla caelata (Conrad 1834)

(synonym of Lemiox rimosus Rafinesque 1820 [97])

Birdwing Pearly Mussel

USA: Alabama (ex), Tennessee, Virginia
$3,6,10,21,33,148,176,209,239$, $244,245,249$ 
Cyprogenia aberti (Conrad 1850)

I1 E

$45,48,138,241$

Western Fanshell Mussel, Edible Pearly Mussel

USA: Arkansas, Kansas (ex?), Missouri [37,47], Oklahoma (ex?)

Dromus dromas (Lea 1834)

I $\quad$ E

$6,10,21,33,129,148,176,209,239$, 244,245

Dromedary Pearly Mussel

USA: Alabama (ex), Kentucky (ex), Tennessee, Virginia

Epioblasma curtisii Utterback 1915

I Id

$6,33,46,47,137,148,162,179,209$, $242,245,264,279$

(synonym of Plagiola florentina (Lea 1857) [137])

Curtis' Pearly Mussel, Curtis' Riffleshell

USA: Missouri

Epioblasma florentina (Lea 1857)

1

E

$6,33,137,148,209,239,241,242$, $245,264,279$

Yellow-blossom Pearly Mussel, Yellow Riffleshell

USA: Alabama (ex), Tennessee

Epioblasma sampsonii (Lea 1861)

I E

$6,56,137,148,209,241,242,266$, 279

Wabash Riffleshell, Sampson's Pearly Mussel, Sampson's Riffleshell

USA (ex?): Illinois, Indiana, Ohio, Tennessee

Epioblasma sulcata perobliqua (Conrad 1836)

$1 \quad \mathrm{E}$

$6,7,130,137,148,209,224,264,279$

(synonym of Dysnomia sulcata (Lea 1829)[97], synonym of Plagiola obliquata (Rafinesque 1820)[137])

White Catspaw Mussel

USA: Indiana, Michigan, ?New York, Ohio

Epioblasma torulosa gubernaculum (Reeve 1865)

$\mathrm{E}$

$6,10,32,33,137,148,176,209,242$, $244,254,264,279$

(synonym of Dysnomia torulosa rangiana (Lea 1839)[97], synonym of Plagiola torulosa (Rafinesque 1820)[137]) Green-blossom Pearly Mussel, Green Riffle Shell

USA: Tennessee, Virginia

Epioblasma torulosa rangiana (Lea 1839)

11

E

$14,33,137,148,209,237,238,241$, $245,246,251,279$

(synonym of Plagiola torulosa (Rafinesque 1820)[137])

Northern Riffleshell, Tan-blossom Pearly Mussel

Canada [53,54]; USA [7,242]

Epioblasma torulosa torulosa (Rafinesque 1820)

I

E

$6,33,48,137,148,209,238,241$, $243,245,249,279$

Tubercled-blossom Pearly Mussel, Northern Riffle Shell, Turberculed Riffle Shell

Canada; USA: Alabama, 1llinois, Kentucky, Tennessee, West Virginia

Epioblasma turgidula (Lea 1858)

I $\quad E$

$6,32,33,128,137,148,179,209$, $239,241,242,245,264,279$

Turgid-blossom Pearly Mussel, Turgid Riffle Shell

USA: Alabama (ex), Arkansas (ex), Missouri (ex), Tennessee 


\section{Fine-rayed Pigtoe Pearly Mussel}

USA: Alabama (ex?), Tennessee, Virginia

Fusconaia edgariana (Lea 1841)

I

E

$3,6,10,21,33,129,148,176,209$,

(synonym of Quadrula cor (Conrad 1834)[97]) $239,244,245$

Shiny Pigtoe Pearly Mussel

USA: Alabama, Tennessee, Virginia

Fusconaia subrotunda (Lea 1831)

$3,129,138,148,176,186,209,239$, 243,244

\section{Long Solid Mussel}

Canada; USA

\section{Lampsilis brevicula (Call 1887)}

II

$47,138,148,209$

(synonym of Villosa reeviana (Lea 1852)[138])

Ozark Broken-ray Mussel, Ozark Lamp Pearly Mussel

USA: Arkansas, Missouri

Lampsilis higginsii (Lea 1857)

1

$6,45,112,113,128,138,148,209$, 263,264

Higgins' Eye Pearly Mussel USA

Lampsilis orbiculata orbiculala (Lea 1836)

I

E

$6,138,148,198,209,239,243,245$, 264

(synonym of Lampsilis abrupta (Say 1831)[97])

Pink Mucket Pearly Mussel

USA

Lampsilis satur (Lea 1852)

I

$138,148,209$

Sandback Pocketbook Mussel, Plain Pocketbook Pearly Mussel

USA: Arkansas [137], Louisiana, Texas

Lampsilis virescens (Lea 1858)

I

E

$6,148,209,239,245,264$

Alabama Lamp Pearly Mussel

USA: Alabama, Tennessee (ex)[33]

Lexingtonia dolabelloides (Lea 1840)

Il

Id

$3,33,129,148,176,209,239,244$, 245

Slab-sided Pearly Mussel

USA: Alabama, Tennessee, Virginia

Plethobasus cicatricosus (Say 1829)

White Warty-back Pearly Mussel

E

USA: Alabama, Indiana (ex), Tennessee 
Plethobasus cooperianus (Lea 1834)

E

(synonym of Quadrula striata (Rafinesque 1820)[97])

Orange-footed Pimpleback Mussel

USA: Alabama, Indiana (ex), Kentucky (ex), Pennsylvania (ex), Tennessee

Pleurobema clava (Lamarck 1819)

II

Id

Clubshell Pearly Mussel

USA

Pleurobema plenum (Lea 1840)

J

E

I

Potamilus capax (Green 1832)

Fat Pocketbook Pearly Mussel

USA

Quadrula intermedia (Conrad 1836)

I

E

E

$6,113,138,148,209$

245,246 245,264

$14,138,148,209,224,237,239,243$

$6,10,33,56,148,176,209,243,244$,

$3,6,10,33,148,176,209,239,245$, 254

Cumberland Monkey-face Pearly Mussel

USA: Alabama (ex), Tennessee, Virginia

Quadrula sparsa (Lea 1841)

I E

$6,10,33,148,176,209,247,264$

(synonym of Orthonymus metanevrus tuberosus (Lea 1840)[97])

Appalachian Monkey-face Pearly Mussel

USA: Tennessee, Virginia

Toxolasma cylindrellus (Lea 1868)

1

E

$6,33,148,209,242,245,248,264$

(synonym of Carunculina glans (Lea 1834)[97])

Pale Lilliput Pearly Mussel

USA: Alabama, Tennessee

Unio nickliniana Lea 1837

1

$6,148,209$

Nicklin's Pearly Mussel

Guatemala; Mexico

Unio tampicoensis tecomatensis Lea 1841

1

$6,148,209$

Tampico Pearly Mussel

Mexico; ?USA: Texas [147]

Villosa trabalis (Conrad 1834)

1

E

$6,33,56,148,176,209,239,240$, 242,244

Cumberland Bean Pearly Mussel

USA: ?Alabama, Kentucky, Tennessee (ex?), Virginia (ex?) 
RL Ref

\section{Class: GASTROPODA}

\section{Order STYLOMMATOPHORA}

\section{Family ACHATINELLIDAE}

The Hawaiian snails of the genus Achatinella from Oahu are collectively known as Little Agate Shells or Oahu Tree Snails. To avoid repetition the main references and common name are listed here only once. Only references to specific taxa are listed for individual species.

Achatinella Swainson 1828

$52,93,98,99,107,108,199,232,26$ 2,279

Little Agate Shell, Oahu Tree Snail

Achatinella abbreviata Reeve 1850

Hawaiian 1slands: Oahu (ex?)

Achatinella apexfulva (Dixon 1789)

Hawaiian Islands: Oahu

Achatinella bellula Smith 1873

Hawaiian Islands: Oabu

Achatinella buddii Newcomb 1853

Hawaiian Islands: Oahu (ex?)

Achatinella bulimoides Swainson 1828

Hawaiian 1slands: Oahu

Achatinella byronii (Wood 1828)

Hawaiian Islands: Oahu

Achatinella caesia Gulick 1858

Hawaiian 1slands: Oahu (ex?)

Achatinella casta Newcomb 1853

Hawaiian Islands: Oahu (ex?)

Achatinella cestus Newcomb 1853

Hawaiian Islands: Oahu (ex?)

Achatinella concavospira Pfeiffer 1859

Hawaiian Islands: Oahu

Achatinella curta Newcomb 1853

Hawaiian Islands: Oahu

Achatinella decipiens Newcomb 1854

Hawaiian Islands: Oahu

Hawaiian Islands: Oahu (ex?)

1

E 
Achatinella dimorpha Gulick 1858

Ex

Hawaiian Islands: Oahu (ex?)

Achatinella elegans Newcomb 1853

Ex

Hawaiian Islands: Oahu (ex?)

Achatinella fulgens Newcomb 1853

E

Hawaijan Islands: Oahu

Achatinella fuscobasis (Smith 1873)

E

Hawaiian Islands: Oahu

Achatinella juddii Baldwin 1895

Ex

Hawaiian Islands: Oahu (ex?)

Achatinella juncea Gulick 1856

Ex

Hawaiian Islands: Oahu (ex?)

Achatinella lehuiensis Smith 1873

Ex

Hawaiian Islands: Oahu (ex?)

Achatinella leucorraphe Gulick 1873

E

Hawaiian Islands: Oahu

Achatinella lila Pilsbry 1914

I

E

Hawaiian Islands: Oahu

Achatinella livida Swainson 1828

Ex

Hawaiian Islands: Oahu (ex?)

Achatinella lorata (Férussac 1824)

E

Hawaiian lslands: Oahu

Achatinella mustelina Mighels 1845

I

$$
\text { E }
$$

100,273

Hawaiian Islands: Oahu

Achatinella papyracea Gulick 1856

I

Ex

Hawaiian Islands: Oahu (ex?)

Achatinella phaeozona Gulick 1856

Ex

Hawaiian Islands: Oahu (ex?)

Achatinella pulcherrima Swainson 1828

E

Hawaiian Islands: Oahu

Achatinella pupukanioe Pilsbry and Cooke 1914

E

Hawaiian Islands: Oahu

Achatinella rosea Swainson 1828

I

Ex

Hawaiian Islands: Oahu (ex?)

Achatinella sowerbyana Pfeiffer 1855

E

Hawaiian Islands: Oahu 
Hawaiian Islands: Oahu (ex?)

Achatinella swiftii Newcomb 1853

Hawaiian Islands: Oahu

Achatinella taeniolata Pfeiffer 1846

1

E

Hawaiian Islands: Oahu

Achatinella thaanumi Pilsbry and Cooke 1914

Hawaiian Islands: Oahu (ex?)

Achatinella turgida Newcomb 1853

Hawaiian Islands: Oahu

Achatinella valida Pfeiffer 1855

$1 \quad$ Ex

Hawaiian Islands: Oahu (ex?)

Achatinella viridans Mighels 1845

Hawaiian Islands: Oahu (ex?)

Achatinella vittata Reeve 1850

1

Ex

Hawaiian Islands: Oahu (ex?)

Achatinella vulpina (Férussac 1824)

Hawaiian Islands: Oahu (ex?)

Family CAMAENIDAE

Papustyla pulcherrima Rensch 1931

II

$\mathbf{R}$

$58,106,200,279$

Manus Green Tree Snail

Papua New Guinea: Manus in the Admirality Islands

Family PARYPHANTIDAE ( = RHYTIDIDAE)

The genus Powelliphanta was, until recently considered to be a subgenus of Paryphanta [60].

Paryphanta annectens Powell 1936

Il

$22,59,163,202,234,270$

Oparara Land Snail

New Zealand: West Nelson

Paryphanta busbyi (Gray 1840)

II

V

$22,23,59,180,202$

Kauri Snail, Pupurangi

New Zealand: Northland

Paryphanta fiordlandica Climo 1971

II

$22,59,61,163,202,234$

New Zealand: South-west Fiordland

Paryphanta gilliesi Smith 1880

II

V

$22,59,61,163,201,202,234$

Gillies' Land Snail

New Zealand: North-west Nelson

Paryphanta hochstetteri (Pfeiffer 1862)

II

V

$22,59,61,163,201,202,234$

Hochstetter's Land Snail

New Zealand: Nelson 
Paryphanta lignaria Hutton 1888

Woodformed Land Snail

New Zealand: North Westland

Paryphanta marchanti Powell 1932

Marchant's Land Snail

New Zealand: North Island

Paryphanta rossiana Powell 1930

Ross' Land Snail

New Zealand: Nelson, Westland

Paryphanta spedeni Powell 1932

Speden's Land Snail

New Zealand: Southland

Paryphanta superba Powell 1930

Superb Land Snail

New Zealand: North-west Nelson

Paryphanta traversi Powell 1930

Travers' Land Snail

New Zealand: Horowhenua in Wellington
II

V

$22,59,61,163,201,202,234,271$

II

$22,59,61,163,201,202,234$

II

V

$22,59,61,163,201,202,234$

II

$22,59,61,163,201,202,234$

II

$22,59,61,163,201,202,234$

II

V

$22,59,61,163,201,202,234$

\section{Order MESOGASTROPODA}

\section{Family STROMBIDAE}

Strombus gigas (Linnaeus 1758)

II

CT

$1,13,25,41,42,57,119,131,170$, Queen Conch, Pink Conch 259,279

(Atlantic Ocean: including Caribbean Sea, Gulf of Mexico)

Anguilla; Antigua and Barbuda; ?Aruba; Bahamas; Barbados; Belize [89]; Bermuda; Brazil; British Virgin Islands; Cayman Islands; Colombia; ?Costa Rica; Cuba; Dominica; Dominican Republic; Grenada [196]; Guadeloupe; Haiti; Honduras; Jamaica; Martinique; Mexico; ?Montserrat; Netherlands Antilles; ?Nicaragua; Panama; Puerto Rico; Saint Kitts and Nevis; Saint Lucia; Saint Vincent; Trinidad and Tobago [196]; Turks and Caicos lslands; USA: Florida; Venezuela; Virgin Islands of the United States 


\section{REFERENCES - NON-CORAL INVERTEBRATES}

1. Abbott, R.T. 1980. The Shell Trade in Florida. Status, Trade and Legislation. Special Report 3. TRAFFIC (U.S.A.), Waahington, D.C.

2. Ackery, P.R. 1975. A guide to the genera and species of Parnassiinae (Lepidoptera: Papilionidae). Bullerin of the British Museum (Noural History). Entomology 31: 71-105.

3. Ahlstedi, S.A. 1983. The molluscan fauna of the Elk River in Tennessee and Alabama. American Malocological Bullerin, 1:43-50.

4. Alcala, A.C. undated. Distribution and abundance of giant clams (Family Tridacnidae) in South-central Philippines. Unpublished.

5. Almada-Villela, P.C. 1988. Checklist of fish and inveriebrates listed in the CITES Appendices. Nature Conservancy Council, UK.

6. Andrus, C.B., Herbst, R.L. and Greenwalt, L.A. 1976. Consening our fish and wildlife herifage. Annual Report FY 1976. U.S. Fish and Wildlife Service.

7. Anon. 1974. Endangered wild animals in Ohio. Ohio Department of Natural Resources, Division of Wildlife.

8. Anon. 1976. Rote Liste bedrohter Tiere in Boyem (Wirbelniere und Inseksen). Schriften der Natur. Lans. Bayern 3: 1-12.

9. Anon. 1977. Tan Riffle Shell determined to be endangered. Department of Interior News Release, U.S. Fish and Wildlife Service, 9 September.

10. Anon. 1982. Virginia's endengered mussels studied by State's Co-op Fishery Resesrch Unit. Endangered Species Technical Bulletin 7(3): 6-7.

11. Anon. 1985. Taiwanese fishing vessel finally brought to justice. Fins 18(1): 3-6.

12. Anon. 1991. European Red List of Globally Threatened Animals and Plants. E/ECE/1249/ECE/ENVWA/20. European Commission for Europe, Geneva.

13. Anon. 1992. Inclusion of Strombus gigas in Appendix II Amendments to Appendices 1 and $I I$ of the Convention. Convention on International Trade in Endangered Species of Wild Fauna and Flora, Eighth Meeting of the Conference of the Parties, Kyoto (Japan), March 1992.

14. Anon. 1992. Clubshell (Pleurobema clava) and Northem Riffleshell (Epioblasma torolusa rangiana) (sic). Endangered Species Technical Bullerin Vol. XVII Nos. 38, p.8.

15. Andt, W. 1940. Als Heilmottel gebrauchte Stoffe Q. Blutegel. Die Rohstoffe des Tierreiches Q. Berlin. Pp. 524-573.

16. Arora, G.S. and Nandi, D.N. 1980. On the butterfly fauns of the Andamsns and Nicobar Islands (India). 1 Papilionidae. Records of the Zoological Survey of India 77: 141-151.

17. Baagoe, P. and Jensen, P. 1985. Supplerrende oplysniger om forekomst at Lsegeigle (Hindo medicinalis L.) i. Danmark Flora og Fauna 91 : 27-30.

18. Bain, J.R. and Humphrey, S.R. 1982. A profile of the Endangered Species of Thailand. Vol.1. Through Birds. Report No. 4, Office of Ecological Services, Florida Stale Museum, Gsinesville. 344 pp.
19. Bannikov, A.G. and Sokolov, V.1. (Eds) 1984. The Red Data Boak of the U.S.S.R. Rare and Threatened Species of Animals and Plants. Lesuaya Promiishlyennost Press, Moscow. (In Rusaian).

20. Barzdo, J. 1985. Order Lepidoptera/Family Papilionidae. Identification Aid to Birdwing Butterfly Species. In: P. Dollinger (Ed.), CITES Idennificarion Manual. Vol.3. Secretsriat of the Convention. Lausanne, Switzerland.

21. Bates, J.M. and Dennis, S.D. 1978. The mussel fauna of the Clinch River, Tennessee and Virginia. Sterkiana 69-70: 3-23.

22. Bell, B.D. 1986. The conservation staus of New Zealand wildlife. New Zealand Wildlife Service, Department of Internal Affairs, Wellington. Occasional Publication 12. 103 pp.

23. Bellingham, M. 1984. What future for Kauri? Forest and Bird 15(3): 14-17.

24. Bennike, S.A.B. 1943. Contributions to the ecology and biology of the Danish freshwater leeches (Hindinea). Folia Limnologica Scandinavica 2: 1-109.

25. Berg, C.J. Jr and Olsen, D.A. 1989. Conservation and management of Queen Conch (Strombus gigas) fisheries in the Caribbean. pp 421-442 ln: Caddy, J.F. (Ed.). Marine Invertebrate Fisheries: their Assessment and Management. John Wiley and Sons, USA.

26. Berger, L.A. 1974. Notes sur quelques Papilionidae du Musee Royal de 1'Afrique centrale. Lambillionea 72-73: 69-76.

27. Bemardi, G., Nguyen, T. and Nguyen, T.H. 1981. Inventaire, carnographie et protection des Lepidopteres en France. Beihefi Veroffentichungen Naturschutz Landschafispflege Baden-Wurtemberg 21: 59-66.

28. Bezobratlí. 1992. Červená Kniha ohrožených a vzácných druhú rastlin a živočichü ČSFR. Príroda bratislava, Czechoslovakia. 155pp.

29. Biedermann, J. 1982. Lebensraum fur Insekten. Liechtensteiner Umwelt bericht June 1982, 4-5.

30. Blab, J. and Kudrna, O. 1982. Naturschurz Akacll, Hilfsprogramm fur Schmetterlinge. Kilda-Verlag, Greven. 135 pp.

31. Bodoy, A. 1984. An assessment of human impact on giant clam populations (Tridacna marima) in the vicinity of Jeddah, Saudi Arabia. Symposia on Coral Reef Environment Red Sea Jeddah. 25 pp.

32. Bogan, A.E. and Parmalee. P.W. 1979. Endangered or Threatened Mollusks of Tennessee. University of Tennessee, Knoxville.

33. Bogan, A.E. and Parmalee, P.W. 1983. The Mollusks, Vol.2. In: Tennessee's Rare Wildlife, Tennessee Heritage Programme, Nashville, Tennessee.

34. Boonsong Lekagul, Askins, K., Nabhitabhata, J. and Samruadkit, A. 1977. Field Guide to the Butterflies of Thailand. Association for the Conservation of Wildlife, Bangkok.

35. Bourgogne, J. 1971. Un temoignage de plus sur la destruction de la nature (Papilionidae). Alexanor 7: 1-50. 
36. Braley, R.D. 1985. Serotonin-induced spawning in gian clams (Bivalvia: Tridacnidae). Aquaculture 47: 321-325.

37. Braley, R.D. 1986. Developmenta in giant clam culture. Australian Fisheries 45(1): 7-9.

38. Bretherton, R.F. and De Worms, C.G. 1963. Butterflies in Corsica 1962. Entomologists' Recond and Joumal of Varionion 75: 93-104.

39. Brown, F.M. and Heinemann, B. 1972. Jamaica and its Butterfies. Classey, London. 478 pp.

40. Brown, J.H. and Muskanofola, M.R. 1985. An investigation of stocks of giant clams (family Tridacnidae) in Java and their utilization and potential. Aquaculture and Fisheries Management 1: 25-39.

41. Brownell, W. 1978. Report on the status of Conch fisheries and related research in Belize, Turks and Caicos, Dominican Republic, Antigua, Dominica, St. Lucia, Barbados, Grenada, Trinidad and Tobago and Venezuela, with notes on three countries not visited (Cuba, Anguilla and Bahamas). Inter-regional Project for the Developmen of Fisheries in the Western Central Atlantic (WECAF), Panama.

42. Brownell, W.N. and Stevely, J.M. 1981. The Biology, Fisheries and Management of the Queen Conch, Strombus gigas. Marine Fisheries Review 43(7):1-12.

43. Bryan, P.G. and McConnell, D.B. 1976. Status of giant clam stocks (Tridacnidae) on Helen Reef, Palau, Westem Caroline Islands, April 1975. Marine Fisheries Review 38: 15-18.

44. Bryk, F. 1935. Lepidoptera Parnassiidae para 11 (Subfamily Pamassinae). Tierreich 65: Li. 790 pp.

45. Buchanan, A.C. 1980. Mussels (Naiades) of the Meramec River Basin, Missouri. Aquatic Series No. 17, Missouri Department of Conservation, Jefferson City Missouri. 68 pp.

46. Buchanan, A.C. 1981. The distribution and habitat of the Curtis' Pearly Mussel, Epioblasma forentina curtisi (Utterback 1915) in south-eastern Missouri. (Abstract). Bulletin of the American Malacological Union 1981: 43.

47. Buchanan, A.C. 1982. A study of Epioblasma florentina curtisi (Utterback 1915), the Curtis Pearly Mussel, in the Upper Little Black River, Missouri. U.S. Department of Agriculture Soil Conservation Service. Unpublished. 11 Pp.

48. Burch, J.B. 1975. Freshwater Unionacean Clams Mollusca: Pelecypada) of North America. Revised Edition, Malacological Publications, Michigan.

49. Burckhardi, D., Gfeller, W. and Miller, H.U. 1980 Animaux proteges de Suisse. Ligue Suisse pour la Protection de la Nature (LSPN). Birkhauser SA, Bale. 224 pp.

50. Calderara, P. 1984. A new subspecies of Omithoptera victoriae Gray (Papilionidae) from Choiseul, Solomon Islands. Proceedings and Transactions of the Brinish Entomological and Natural History Society 17: 31-35.

51. Caputa, A., Holcik, J. and Berger, Z. 1982. Allas of protected animals in Slovakio. Obzor, Bratislava. 434 pp.

52. Chambers, S.M. and Williams, L.K. 1980. Endangered and threatened wildlife and plants. Proposed Endangered status for Achatinella, a genus of Hawaiian tree snails. Federal Register 45(125): 43358-43360.
53. Clarke, A.H. 1973. On the distribution of Unionidae in the Sydenham River, Southem Ontario. Malacological Review 6: 63-64.

54. Clarke, A.H. 1976. The endangered mollusca of Canada. In: Mosquin, T. and Suchal, C. (Ede), Canada's Threatened Species and Habilats. Proceedinge of the Symposium, May 1976. Ottawa, Canada.

55. Clarke, A.H. 1981. Notes on the names Dysnomia, Epioblasma, and Plogiola. In: Determination of the precise geographical areas occupied by four endangered species of freshwater molluscs. Final Report to U.S. Fish and Wildlife Service. Twin Citica, Minnesota. Contract No. 14-16-003-81-019.

56. Clarke, A.H. 1983. The diatribution and relative abundance of Lithasia pinguis (Lea), Pleurobema plenum (Lea), Villosa trabalis (Conrad) and Epioblasma sampsoni (Lea). American Malacological Bulletin, 1:27-30.

57. Clench, W.J. and Abbott, R.T. 1941. The genus Strombus in the Western Atlantic. Johnsonia 1:1-15.

58. Clench, W.J. and Turner, R.D. 1962. Monographs of the genera Papustyla, Forcartia, and Meliobba Papuininae: Camaenidae). The Malacologic al Society of Australia.

59. Climo, F.M. 1975. The land snail fauna. In: G. Kuschel (Ed.), Biogeography and Ecology in New Zealand. Junk, The Hague. 689 pp

60. Climo, F.M. 1976. A new higher classification of New Zealand Rhytididae (Mollusca: Pulmonata). Joumal of the Royal Society of New Zealand 7(1): 59-65.

61. Climo, F.M. 1978. The Powelliphanta gilliesi - traversi hochsteueri - rossiana - lignaria - superba ring species (Mollusca: Pulmonata). New Zealand Joumal of Zoology (5): 289-294

62. Collins, N.M. 1987. Butterfly Houses in Britain - The Conservation Implications. Unpublished report. IUCN, Cambridge. 60 pp. +4 annexes.

63. Collins, N.M. and Morris, M.G. 1985. Threatened Swallowtail Butterfies of the World. The IUCN Red Data Book. IUCN, Gland and Cambridge. vii $+401 \mathrm{pp}$.

64. Collins, N.M. and Wells, S.M. 1987. Invertebrales in need of special protecrion in Europe. Council of Europe. Strasbourg. Nature and Environment Series 35: $162 \mathrm{pp}$.

65. Common, 1.F.B. and Waterhouse, D.F. 1972. Bunerflies of Australia. Angus and Robertson, Sydney. 498 pp.

66. Corbet, A.S. and Pendlebury, H.M. 1978. The Butterflies of the Malay Peninsula. Third edition revised by J.N. Eliot. Malayan Nature Society, Kuala Lumpur. 578 pp.

67. Crawford, C.M., Nash, W.J. and Lucas, J.S. 1986 Spawning induction, and larval and juvenile rearing of the Giant Clam, Tridacna gigas. Aquaculure 58: 281-295.

68. Cristea, V. and Manoleli, D. 1977. Conspectus des sangsues (Mindinea) de Roumanie avec une clef de determination. Travaux dus Muséum d'Histoire Naturelle "Gr. Anripa" 18: 23-56.

69. D'Abrera, B. 1971. Butterflies of the Australian Region. Lansdowne Press, Melboume. 415 pp.

70. D'Abrera, B. 1975. Birdwing Butterflies of the World. Lansdowne Press, Melboume. 415 pp.

71. D'Abrera, B. 1981. Butterflies of the Neotropical Region Part 1. Papilionidae and Pieridae. Lansdowne Editions, Melboume. 172 pp. 
72. D'Abrera, B 1982. Butterflies of the Oriensal Region. Pan 1. Papilionidae and Pieridac. Hill House, Victoria, Australia. 244 pp.

73. Dabrowaki, J.S. 1975. Some problems in the preservation of butherflies in Poland. Atala 3: 45.

74. Dabrowaki, J.S. 1980. The protection of the Lepidopterofaun - the latest trends and problems. Nata Lepidopteranum 3: 114-118.

75. Dabrowski, J.S. 1980. The disappearance of the biotopes of Pamassius apollo (L.) in Poland and the neceasity of ita active preservation (Lepidoptera, Papilionidae). Casopis Slezsk ho Musea v Opave. Opava. Ser. A. Historia Naturalis 29: 181-185. (In Polish).

76. Darby, A.W. 1985. On the statua of Omithoptera meridionalis tanunggarensis Joicey and Talbot. Papilio Intemational 12(3-4): 119-125.

77. Debout, G. and Provost, M. 1981. Le Marais de la Sangsuri ve. Le Courrier de la Narure 74: 10-18.

78. Deslisle, G. 1985. Nouvelle sous-espece chez Omithoptera tithonus de l'ile Misool, Indonesie (Lepidoptera: Papilionidae). Canadian Entomologist 117: 221-225.

79. Dresscher, T.G.N. and Highler, L.W.A. 1982. De Nederlandse Bloedzuigers. Hirundinea. Wetenschappelijke Mededelingen Koninklijke Nederlandse Naturhistorische Vereniging 154: $64 \mathrm{pp}$.

80. Elliott J.M. and Tullen, P.A. 1982. Provisional Atlas of the Freshwater Leeches of the British Isles. Freshwater Biological Association, Occasionsl Publication 14: 1-31.

81. Elliott, J.M. and Tullett, P.A. 1984. The Status of the Medicinal Leech Hinudo medicinalis in Europe and especially in the British Isles. Biological Conservation 29: 15-26.

82. Elliott, J.M. and Tullett, P.A. 1986. The effects of temperature, atmospheric pressure and season on the swimming activity of the Medicinal Leech, Hinudo medicinalis (Hinudinea; Hinudinidae), in a Lake District Lam. Freshwater Biology 16: 405-415.

83. Emmel, T.C. and Garraway, E. 1990. Ecology and Conservation Biology of the Homerus Swallowrail in Jamaica (Lepidoptera: Papilionidae). Tropical Lepidoptera 1(2):63-76.

84. Evana. W.H. 1932. The Idennification of Indian Butterflies. Bombay Natural History Society. Second Edition, revised. 454 pp.

85. Fausser, J. 1980. Observations concemsnt Papilio hospiton Gene en Haute-Corse. Bulletin Laison l'Associarion Entomologique d'Evreur 5: 18-19.

86. Fausser, J. 1988. Informations complémentaires sur Papilio hospiton en Haute-Corse. Alexanor 15:447-448.

87. Forselius, S. 1952. Blodigelu (Hirudo medicinalis L.) i Norden. Sartryck ur Sr. Foun. Rery 3: 67-79.

88. Fry, 1. and Robinson, M. 1986. The threatened Invertebrates. In: Kennedy, M. and Burton R. (Eds), A Threatened Species Consenvarion Strategy for Australia. Ecofund Australia: 14-17.

89. Gibson, J., Strasdine, S. and Gonzales, K. 1983. The status of the Conch industry of Belize. Proceedings of the Gulf and Caribbean Fisheries Instituke, No 35. Gulf and Caribbean Fisheries Institule, Miami, Florida.
90. Gomez Bustillo, M.R. and Fernandez-Rubio, F. 1974 Mariposas de la Peninsula lberica (tomo 1). Servicio de Publicacioner del Ministerio de Agricultura, Madrid. 198 pp.

91. Gomez Bustillo, M.R. and Fernandez-Rubio, F. 1974. Mariposas de la Peninsula lberica (tomo 2). Servicio de Publicaciones del Ministerio de Agricultura, Madrid. 258 pp.

92. Gabriel, A.G. 1942. A new species of Bhuranitis (Lep. Papilionidae). Entomologist 75: 189.

93. Gagné, B.H., Kay, E.A. and Longford, P.S. 1975. A aurvey of Achatinella on Oahu, Hawaii, September-December 1974. Report to Office of Endangered Species, USDI.

94. Gepp, J. 1983. Rote Listen Gefahndeter Tiere Osterreichs. Bundeaministeriums fur Gesundheit und Umweltschutz, Wien. 242 pp.

95. Giben, J.M.M. 1969. Pamassius apollo in the eastern Spanish Pyrennees. Tieg Newsletter 10(2): 16-20.

96. Goreau, T.F., Goreau, N.J. and Youge, C.M. 1973. On the utilization of photosynthetic products from zooxanthellae and of a dissolved animo acid in Tridacna maxima f. elongata (Mollusca: Bivalvia). Joumal of Zoology 169: 417-454

97. Hass, F. 1969. Superfamilia Unionacea. Das Tierreich 88: $1-663$.

98. Hadfield, M.G. 1982. Field studies of Oahu's native tree snails. 4th Conference in Natural Sciences. 2-4 June 1982.

99. Hadfield, M.G. 1986. Extinction in Hawaiian Achatinelline snails. Malacologia 27(1): 67-81.

100. Hadfield, M.G. and Mountain, B.S. 1980. A field study of a vanishing species, Acharinella mustelina (Gastropoda: Pulmonata), in the Waianae Mountains of Oahu. Pacific Science 34(4): 345-358.

101. Hammner, W.M. and Jones, M.S. 1976. Distribution. burrowing and growth rates of the clam Tridacna crocea on interior reef flats. Oecalagia (Berl.) 24: 207-227.

102. Hancock, D.L. 1982. A note on the status of Omithoptera meridionalis tarunggarensis (Joicey and Talbot) (Lepidoptera: Papilionidae). Australian Entomological Magazine 8: 93-95.

103. Hancock, D.L. 1983. Classification of the Papilionidae (Lepidoptera): a phylogenetic approach. Smithersia 2: 1-48.

104. Harada, M. 1965. The capture of Papilio chikae. Tyo To $\mathrm{Ga}$ (Transactions of the Lepidopterists' Society of Japan) 16: 48-49.

105. Hardy, J.T. and Hardy, S.A. 1969. Ecology of Tridacna in Palau. Pacific Science 23: 467-472.

106. Harrison Gagné, B. 1981. Up a tree with the Manus Green Snail. Hawoiion Shell News 24(5) N.S. 257: 1, 8-9.

107. Han, A.D. 1978. The onslaught against Hawaii's tree snails. Naturol History 87(10): 46-57.

108. Hart, A.D. 1979. A survival status report on the endemic Hawaiian tree snsil genus Achatinella from Oahu. Report to OES, U.S. Department of the Interior.

109. Harvey, R. (Ed.) 1986. The Cambridge Palawan Expedition 1985. Final Report. Unpublished. 
110. Haugum, J. snd Low, A.M. 1978-1983. A Monograph of the Birdwing Butterflies 1(1): 1-84; (2): 85-192; (3): 193-308. 2(1): 1-104; (2): 105-240. Scondinavian Science Press, Klampenborg.

111. Hougum, J. and Low, A.M. 1985. A monograph of the birdwing butterflies. Vol. 2(3). Troides helena and aeacus groupa. E.J. Brill, Leiden, Holland and Scandinavian Science Preas, Klampenborg, Denmark. Pp 241-356.

112. Havlik, M.E. 1983. Naiad mollusk populations (Bivalvia: Unionidae) in pools 7 and 8 of the Mississippi River near la Crosse, Wisconsin. American Malacological Bulletin, $1: 51-59$.

113. Havlik, M.E. snd Stansbery, D.H. 1977. The naiad mollusks of the Mississippi River in the vicinity of Prairie du Chien, Wisconsin. Bulletin of the American Malacological Union 1977: 9-12.

114. Heath, J. 1981. Threatened Rhopalocera (Butterflies) in Eurape. Council of Europe, Nature and Environment Series 23: $157 \mathrm{pp}$.

115. Heath, J. and Leclerg, J. (Eds) 1981. European Invertebrate Survey. Provisional Allas of the Invertebrates of Europe, Maps 1-27. Institute of Terrestrial Ecology, Monks Wood and Facult des Sciences Agronomiques, Gembloux.

116. Herter, K. 1968. Der medizinische Blusegel und seine Venwandie. Die Neue Brehm-Bucherei, No. 381. Berlin, Wittenberg Lutherstadt, A. Ziemsen Verlag.

117. Heslinga, G.A., Perron, F.E. and Orak, O. 1984. Mass culture of giant clams (F. Tridacnidae) in Palau. Aquaculture 39: 197-215.

118. Heslinga, G.A. and Watson, T.C. 1985. Recent advances in giant clam mariculture. Proceedings of the 5 th Intemational Coral Reef Congress, Tahiti 5: 531-537.

119. Hesse, K.O. 1979. Movement and migration of the Queen Conch, Strambus gigas, in the Turks and Caicos Islands Bulletin of Marine Science 29(3):303-311.

120. Higgins, L.G. and Riley, N.D. 1980. A Field Guide to the Butterflies of Britain and Europe. 4th edition revised Collins, London. $384 \mathrm{pp}$.

121. Hirschberger, W. 1980. Tridacnid clam stocks of Helen Reef, Palau, Western Carolina Istands. Marine Fisheries Review 42: 8-15.

122. Hoffman, J. 1955. Faune hirudinéenne du Grand-Duche de Luxembourg. Instilut Grand-Ducal, Section des Sciences Naturelles, physiques et mathematiques, Archives (2)22: 200-202.

123. Hoffman, J. 1955. Signalement d'une importante station de Hiruda medicinalis L. au Grand-duche de Luxembourg. Institut Grand-Ducal, Section des Sciences naturelles, physiques et maihematiques, Archives (2)22: 213-222.

124. Hoffman, J. 1960. Notules hirudinologiques, 2. Nouvelle Station de Hinudo medicinalis au Grand-Duch. Insriuu Grand-Ducal. Section des Sciences naurelles, physiques et mathematiques, Archives (2)27: 289

125. Holloway, J.D. 1978. Butterfies and moths. In Kinabalu Summit of Bomeo. Sahah Sociely Monograph, 25-278.

126. Igarashi, S. 1965. Papilio chikac, an unrecorded Papilionid butterfly from Luzon island, the Philippines. Tyo To Ga (Transactions of the Lepidopterists' Society of Japan) 16: $41-49$.
127. Igarashi, S. 1979. Papilionidae and their early stages. (1): 219 pp.; (2): 102 pp of plates. Kodansha, Tokyo. (In Japanese).

128. Imlay, M.J. 1977. Competing for survival. Water Spectrum 9(2): 7-14.

129. Imlay, M.J. 1982. Use of shells of freshwater mussels in monitoring heavy metals and environmental stresa: review. Malacological Review 15: 1-14.

130. Isom, B.G., Gooch, C. snd Dennis, S.D. 1979. Rediscovery of a presumed extinct river mussel, Dysnamia sulcata (Unionidse). Nautilus 93(2-3): 84.

131. Iversen, E.S., Jory, D.E. and Bannerot, S.P. 1986. Predation on Queen Conchs, Strombus gigas, in the Bahamas. Bulletin of Marine Science 39(1):61-75.

132. Janzon, L.-A. 1990. The distribution of Pamassius apallo (L) in Sweden. Entomologists" Gazetie 41:82-83.

133. Janzon, L.A. and Bignert, A. 1979. Apollofjarilen i Sverige. Fauna Flora, Uppsala 74: 57-66.

134. Jazdzewska, T. 1983. Additional information on the status of the medicinal leech, Hinudo medicinalis $\mathbf{L}$. in Poland and USSR. Unpublished report to IUCN. 4 pp.

135. Jensen, B. 1960. Laegeiglens (Hirudo medicinalis L.) forekomst i Danmark. Flora Fauna 66: 25-32.

136. Jiménez, J.M. and Garcia-Mas, I. 1980-81. Hirudineos de Espana: catalogo provisional. Boletim Saciedade Partuguesa de Ciencias Naturais 20: 119-125.

137. Johnson, R.I. 1978. Systematics and zoogeograhy of Plagiola $(=$ Dysnomia $=$ Epioblasma $)$, an almost extinct genus of freshwater mussels (Bivalvia: Unionidae) from middle North America. Bulletin of the Museum of Comparative Zoology 148(6): 239-320.

138. Johnson, R.1. 1980. Zoogeography of North American Unionacea (Mollusca: Bivalvia) north of the maximum Pleistocene glaciation. Bulletin of the Museum of Comparative Zoology 149(2): 77-189.

139. Johnston, G. and Johnston, B. 1980. This is Hong Kong Butserflies. Crown copyright, Hong Kong. 224 pp.

140. Jumalon, J.N. 1967. Two new papilionids. Philippine Scientist 1(4): 114-118.

141. Jumalon, J.N. 1969. Notes on the new range of some Asiatic papilionids in the Philippines. Philippine Entomologist 1(3): 251-257.

142. Keve, 1968. Ueber die Arealveranderungen von Plegadis falcinellus (L.). Znologische Abhandlungen Staatliches Museum fur Tierkunde in Dresden 29(13): 169.

143. Kobayashi, H. and Koiwaya, S. 1978. A new species of Omithoptera (Lepidoptera: Papilionidae) from West Irian. Transactions of the Himeji Naural History Assaciation (special issue) $17 \mathrm{pp}$.

144. Kobayashi, H. and Koiwaya, S. 1981. Troides celebensis status nova, with descriptions of its new subspecies. Transactions of the Himeji Natural Histary Association $1981: 21-26$.

145. Koubkova, B. and Vojtkova, L. 1973. Zur Kenntnis der Tschechoslowakischen Hirudineenfauna. Folia Facultatis Scientiarum Naturalium Universitatis Purkynianae Brunensis Biologia 14: 103-118. (In Czech with German summary). 
146. Kudrna, O. 1986. Grundlagen zu einem Artenschutzprogramm fur die Tagschmetterlingsfaura in Bayern und Analyse der Schutzproblematik in der Bundersrepublik Deutschland. Nachrichten ent. Verein fur Apalla, Frankfurt, Suppl. 6: 1-90.

147. Landye, J.J. 1980. Staus of rare, endangered and/or threatened molluscan species of Texas and Oklahoma. Report to U.S. department of the Interior, U.S. Fish and Wildlife Service, Albuquerque, New Mexico. Contract (1) 16-0002-79-202.

148. Loycock, G. 1983. Vanishing Naiads. Audubon 85(1): 26-28.

149. Lent, C. 1986. New medicinal and scientific uses of the leech. Narure 323: 494.

150. Loffler, H. 1974. Die Kleintierfauna des Schilfgrtels. In: Der Neusiedlersee. Chapter 12. Verlsg Fritz Molden. Wien.

151. Lopez, M.D.G. and Heslings, G.A. 1985. Effect of dessication on Tridacna derasa seed: implications for long distance transport. Aquaculture 49: 363-367.

152. Low, A.M. and Haugum, J. 1983. Traganoptera broakiana natunensis Rothsch. 1908. Papilio Intemational I(1): $11-15$.

153. Lucas, J.S., Ledua, E. and Braley, R.D. 1990. A New Species of Giant Clam (Tridacnidae) from Fiji and Tonga. ACIAR Working Paper No. 33, Australian Centre for International Agricultural Research, Canberra, 8pp.

154. Lukin, E.I. 1957. On the distribution of the medicinal leech in the U.S.S.R. Zoologicheskii Zhumal 36: 658-669. (In Russian).

155. Lukin, E.1. 1976. Leeches of fresh and brackish water-bodies. In: Fauna of the U.S.S.R. 1. Leningrad, Nauka.

156. Mrcfsrlane, R. 1985. Insect farming and trading - Solomon Islands. Papilio International 2(3-4): 127-129.

157. Mrndal, D.K. 1984. Notes on the Papilionidse of Arunachal Pradesh, North-east India. Papilia Intemational I(4): 76-81.

158. Maquet, B. 1985. La sangsue medicinale, Hinua medicinalis (L.), une especc dont le statut est incertain en Belgique. Les Naturalistes Belges 66(2): 32-42.

159. McCarthy, T.K. 1975. Observations on the distribution of the freshwster leeches (Hirudinea) of Ireland. Praceedings of the Royal Irish Academy 75B: 401-451.

160. McKoy, J.L. 1980. Biology, exploitation and management of giant clams (Tridacnidae) in the Kingdom of Tonga. Fisheries Bulletin Tonga (1): 61 .

161. McMichael, D.F. 1975. Growth rate, population size and mantle colouration in the Small Giant Clam Tridacna maxima (Röding) at One Tree Island, Capricom Group, Queensland. In: Praceedings of the 2nd Intermational Coral Reef Sympasium 1. Great Barrier Reef Committee, Brisbane: 241-254.

162. McMillan, W. 1979. Channelization threatens otters, mussels, Little Black. Ozark Guardian September 1979: 2-3.

163. Meads, M.J., Walker, K.J. and Elliott, G.P. 1984. Status, conservation, and management of the land snails of the genus Powelliphanta (Mollusca: Pulmonata). New Zealand Joumal of Zoology 11: 277-306.
164. Mell, R. 1938. Beiträge zur Fauna Sinica. Deutsche Entamalagische Zeitschrift 17: 197-345.

165. Mikkola, K. 1981. Extinct and vanishing Lepidoptera in Finland. Beihefi Veroffentichungen Naturschutz Landschafisplege Baden-Wurttemberg 21: 175-176.

166. Miller, J.S. 1987. Phylogenetic studies in the Papilioninae (Lepidoptera: Pspilionidae). Bulletin of the American Museum of Natianal Histary 186(4):365-512.

167. Minelli, A. 1977. Irudinei (Hirudinea). Guide per il reconoscimento della specie animali delle acque inteme Italiane. Verona, Consiglio Nazionale delle Ricerche.

168. Minelli, A. 1979. Sanguisughe d'Italia. Catalogo orientativo e conaiderazioni biogeografiche. Lavari della Societa Italiana di Biageografia. Forli N.S. 4: 279-313.

169. Mitchell, G.A. undated. The Natianal Butterflies of Papua New Guinea. Wildlife Branch Department of Natural Resources, Papua New Guinea. 16 pp.

170. Mitton, J.B., Berg, C.J. Jr and Orr, K.S. 1989. Population structure, larval dispersal and gene flow in the Queen Conch (Strombus gigas) of the Caribbean. Bialagical Bulletin (Woods Hole) 177(3):356-362.

171. Munro, J.L. 1983. Giant clams - food for the future? ICLARM Newsletter 6(1): 3-4.

172. Munro, J.L. 1986. Status of giant clam stocks and prospects for clam mariculture in the central Gilben Islands graup, Republic of Kiribati. ICLARM Report to the Fisheries Division, Ministry of Natural Resources Development, Kiribati and the South Pacific Regional Fisheties Development Programme, UNDP, SUVA, Fiji.

173. Munro, J.L. 1989. Fisheries for giant clams (Tridacnidae: Bivalvia) and prospects for stock enhancement.pp 541-558 In: Caddy, J.F. (Ed.). Marine Invenebrate Fisheries: their Assessment and Management. John Wiley and Sons, USA.

174. Munro, J.L. and Heslinga, G.A. 1983. Prospects for the commercial cultivation of Giant Clams (Bivalvia: Tridacnidee). Praceedings of the Gulf and Caribbean Fisheries Institute $35: 122-134$.

175. Murzin, V.S. 1981) Parnassius apallo, Map 106. In K.B. Gorodkova (Ed.) Distribution of Insects - European part of the USSR - Allas. Maps 73-125. Leningrad Science. Academy of Science, Zoological Institute, Leningrad. (In Russian).

176. Neves, R.J., Pardue, G.B., Benfield, E.F. and Dennis, S.D. 1980. An Evaluation of Endangered Mollusks in Virginia. Virginia Commission of Game and Inland Fisheries, Fish Division. Final Report. Project No. E-F-J. 140 pp.

177. New, T.R. and Collins, N.M. 1991. Swallowtail Butserfies: an actian plan for their Conservation. IUCN/SSC Lepidoptera Specialist Group, IUCN, Gland, Switzerland.

178. Nilssen, J.P. 1980. Acidification of a small watershed in southem Norway and some characteristics of acidic aquatic environments. Intemationale Revue des Gesamien Hydrobialogie 65: 177-207.

179. Nordstrom, G.R., Pflieger, W.L., Sadler, K.C. and Lewia, W.H. 1977. Rare and Endangered Species of Missouri. Missouri Department of Conservation and U.S. Department of Agriculture Soil Conservation Service. 
180. Ogle, C.C. 1979. Critical status of Placostylus and Paryphanta land anaila in the Far North. New Zealand Wildlife Service, Wellington. Fauna Survey Unit Report 14: $1-6$.

181. Okano, K. 1983. Some ecological notes on Teinopalpus. Tokurana (Acta Rhopalocerologica) 5: 94-110. (In Japanese).

182. Okano, K. 1984. Color illustration of Bhutanitis mansfieldi (Riley 1940) (Papilionidae): with some notes on the same speciea. Tokurana (Acta Rhopalocerologica) 6/7: 61-65.

183. Palik, E. 1980. The protection and reintroduction in Poland of Pamassius apollo (Linnaeus) (Papilionidae). Nota Lepidopterologica 2: 163-164.

184. Palik, E. 1981. The conditions of increasing menace for the existence of certain Lepidoptera in Poland. Beihefi Veroffentlichungen Naturschutz Landschafispflege Baden-Wurttemberg 21: 31-33.

185. Not used.

186. Parmslee, P.W. and Klippel, W.E. 1984. The niaid fauna of the Tellico River, Monroe County, Tennessee. American Malacological Bulletin, 3(1):41-45.

187. Parrot, R.E. 1985. A new subspecies of Omithopera priamus, Linn. from Gebe Island, Indonesia. Papilio Intemational 2(3-4): 131-142.

188. Parrott, R.E. 1991. New Borneo (Kalimantan) subspecies of Troides and Trogonoptera (Lepidoptera: Papilionidae). Tropical Lepidoptera 2(2): 122-136.

189. Parrott, R.E. and Deslisle, G. 1986. New and interesting forms of birdwing butterflies. Part 1, Omithoptera, subgenus Schoenbergia. Papilio Intemarional 2 Supplement: 147-168.

190. Parsons, M.J. 1983. A conservation study of the birdwing butterflies Omithoptera and Troides (Lepidoptera: Papilionidae) in Papua New Guinea. Final Report to the Department of Primary Industry, Papua New Guinea. 111 pp.

191. Parsons, M.J. 1992. The World's Largest Butterfly Endangered: the Ecology, Status and Conservation of Omithoptera alexandrae (Lepidoptera: Papilionidae). Tropical Lepidoptera 3 (Suppl. 1):33-60.

192. Pastermak, J. 1981. On the rediscovery of Omithoptera meridionalis tarunggarensis Joicey and Talbot on a new locality in Kamrau Bay, South West Irian Jaya, Indonesia. Transacrians of the Himeji Natural History Association 1981: $2-14$.

193. Pastemak, J. 1986. Western Omithoptera (Schoenbergia) meridionalis two forms of subspecies tarunggarensis?. Papilia Intemational 3(1-2): 185-189.

194. Pearson, R.G. 1977. Impact of foreign vessels poaching giant clams. Australian Fisheries 36(7): 8-11

195. Pen, D. 1936. The Papilionidae of south-westem Szechwan. Joumal of the West China Bonder Research Sociery 8: 153-165.

196. Perchade, P.L. 1982. A comparison of the Strombus (Mollusca) colonies, of two southern Caribbean Islands Trinidad and Grenada. Caribbean Joumal of Science 18(14): $35-40$.
197. Pernetta, J. 1987. Giant clams: a new potential food source in tropical small ialand statea or another source of biological contamination? Science in New Guinea 13(2): 92-96.

198. Pflieger, W.L. 1974. Animal Kingdom. In: Rare and Endangered Species of Missouri. Missouri Department of Conservation and U.S. Department of Agriculture and Soil Conservation Service.

199. Pilsbry, H.A. and Cooke, C.M. Jr. 1912-1914. Achatinellidae. Manual of Conchology 2(22).

200. Pitman, R.W. 1977. Manus Island'a green tree snails at home. Hawaitian Shell News 25(4), N.S. 208: 9-10.

201. Powell, A.W.B. 1976. Shells of New Zealand. Sth revised edition. Whitcoulls Ltd., Christchurch, New Zealand.

202. Powell, A.W.B. 1979. New Zealand Mallusca: marine, land and freshwater shells. Collins, Auckland. 500 pp.

203. Pyle, R.M. and Hughes, S.A. 1978. Conservation and utilisation of the insect resources of Papua New Guinea. Report of a consultancy to the Wildife Branch, Department of Nature Resources, Independent State of Papua New Guinea. 157 pp. Unpublished.

204. Racheli, T. 1980. A list of the Papilionidae (Lepidoptera) of the Solomon Islands, with notes on their geographical distribution. Australian Entomological Magazine 7: 45-59.

205. Racheli, T. 1984. Further notes on Papilionidae from the Solomon Islands. Papilio Intemational 1: 55-63.

206. Rákosy, L. 1983. Problema ocrotirii Lepidopterelor in Romania, exemplificari din Judetul Cluj. Ocronirea Naturii med. inconj. l. 27: 32-36. Bucuresti.

207. Ramadoss, K. 1983. Giant clam resources. In: K. Alagarswami (Ed.), Mariculture potential of the Andaman and Nicobar Islands - an indicative survey. Central Marine Fisheries Research Institute (CMFRI) Bullerin 34: 108.

208. Riley, N.D. 1975. A Field Guide to the Butterflies of the West Indies. Collins, London. 244 pp.

209. Roberts, R.J. 1983. Saving the freshwater mussel. Nature $303(3): 13$

210. Robinson, J.C. 1975-1976. Swallowtail butterflies of Sabah. Sabah Society Joumal 6: 5-22.

211. Rosewater, J. 1965. The family Tridacnidae in the Indo-Pacific. Indo-Pacific Mollusca l(6): 347-396.

212. Rosewater, J. 1982. A new species of Hippopus (Bivalvia: Tridacnidae). Nautilus 96(1): 3-6.

213. Russev, B. and Janeva, 1. 1976. Review of the specific composition, distribution, ecology and index significance of leeches in Bulgaria. Hhidrobialogiya, Sofia 3: 40-56.

214. Russev, B. and Marinov, T. 1964. Uber die Polychaten und Hirudineen-Fauna im bulgarischen Sektor der Donau. Izvesnya na Zoologicheskiya Insnitut. Bulgarska Akademiya na Naukite. Sofiya 15: 191-197. (In Bulgarion).

215. Saigusa, T. and Lee, C,-L. 1982. A rare papilionid butterfly Bhutaniris mansfieldi (Riley), its discovery, new subspecies and phylogenetic position. Tya to Ga (Joumal of the Lepidopterists' Society of Japan) 33: 1-24.

216. Sala, G. 1987. A rew form of Papilio hospiton Géne 1831. Papilio Intemarional 3(4):210-211. 
217. Salm, R.V. 1981. Heads we swim, tails we lose. Conservation Indonesia 5(3-4): $12-14$

218. Salvat, B. 1969. Dominance biologique de quelques mollusques dans lea atolla fermes (Tuamotu, Polynerie): phemomene recent consequence 8 actuelles. Malacologia 9: 187-189.

219. Sapkarev, J.A. 1970. The fauna of Hirudinea of Macedonia. The taxonomy and diatribution of leechea of Aegean lakes. Intemationale Revue der Gesamien Hydrobiologie und Hydrogeographie. Leipzig 55: 317-324.

220. Sawyer, R.T. 1976. The medicinal leech Hirudo medicinalis L., an endangered species. In: Forsythe, D.M. and Ezell, W.B. Jr. (Eds), Proceedings of the First South Carolina Endangered Species Symposium. November 11-12, Charleston, S. Carolina. 103-106 pp.

221. Sawyer, R.T. 1981. Why we need to save the Medicinal Leech. Oryx 16(2): 165-168.

222. Sawyer, R.T. 1986. Leech Biology and Behaviour. Volume II. Clarendon Press, Oxford, 430 pp.

223. Sawyer, R.T. and Leake, L.D. (Eds) 1986. International Conference. Leech Newslener (British Association of Leech Scientists) 1 .

224. van der Schalie, H. 1975. An ecological approach to rare and endangered species in the Great Lakes region. Michigan Academy 8(1): 7-22.

225. Scofield, A.M. 1981. A Checklist of the Helmint' Parasites of Domestic Animals in the United Kingdom. Hoechst U.K., Ld., (Animal Health Division), Milton Keynes.

226. Severns, R.M. 1981. Growth rate of Achatinella lila, 8 Hawaiian tree snail. Noutilus 95(3): 140-143.

227. Shirozu, T. 1960. Butterflies of Formosa in Colour. Hoikusha. Osaka, Japan (in Japanese). 483 pp.

228. Sineva, M.V. 1944. (Observations on breeding the medicinal leech). Zoologicheski Zhumal. Moskva 23(6): 293-303.

229. Smith, C. 1975. Commoner Butterflies of Nepal. Tribhuvan Univeraity, Kathmandu, Nepal. 38 pp.

230. Smith, C. 1978. Scientific List of Nepal's Butterflies. Joumal of Natural History Museum 2(3): 127-185.

231. Smith, R.L., Sleeman, J.M., Haworth, R.I. and Batchelor, J.H. 1992. Euathlus smithii, the Mexican Red-Kneed Tarantula; observations in the wild with comments on status and conservation. In Cooper, J.E., Pearce-Kelly, P. and Williams, D.L. (Eds) Arachnida: Proceedings of a Symposium on Spiders and their Allies. London.

232. Solem, A. 1990. How many Hawaiian land snail species are left? - and what we can do for them. Bishop Museum Occasional Papers 30:27-40.

233. Spitzer, K. 1983. Seasonality of the butterfly fauna in southeastern Vietnsm (Papilionoidea). Joumal of Research on the Lepidoptera 22(2): 126-130.

234. Spurr, E.B. 1985. Distribution and abundance of large land snaila. In: M.R. Davis and J. Orwin (Eds), Report on a survey of the proposed Wapiti area, West Nelson. FRI Bulletin 84: 203-209.

235. Stahl, G. 1979. Ripponia hypolitus Cramer. A description of the form 'antiope' from Halmahera, and a new form from Obi. Lepidoptera Group of 68 Newsletler II(5): 135-139.
236. Stsnkovic, S. 1960. Hirudinea. In: The Balkan Lake Ohrid and its living world. Chapter 5. Biogeography. Monographiae Biologicae, 9. The Hague, Junk.

237. Stanabery, D.H. 1961. A century of change in the naiad population of the Scioto River ayatem in central Ohio. Annual Reports of the American Malacological Union: 20-22.

238. Stansbery, D.H. 1964. The Molluscan Fauns. In: Prufer, O.H. et al. The McGraw Site - a study in Hopewellian Dynamics New Series, Vol. 4, No. 1 - Cleveland Museum of Natural Hiatory.

239. Stansbery, D.H. 1964. The Mussel (Muscle) Shoala of the Tennessee River revisited. Annual Reparts af the American Malacological Union 1964: 25-28.

240. Stansbery, D.H. 1969. Changes in the Naiad fauna of the Cumberland River at Cumberland Falls in Eastem Kentucky. Annual Reports of the American Malacological Union 1969: 16-17.

241. Stansbery, D.H. 1970. Eastern freshwater mollusks (1). The Mississippi and St. Lawrence River systems. In Clark, A.H. (Ed.), Papers on rare and endangered mollusk of North America. Malacologia 10(1): 9-20.

242. Stansbery, D.H. 1971. Rare and endangered mollusks in the Eastern United States. In: Jorgensen, S.E. and Sharp, R.W. (Eds), Proceeding of a Symposium on the rare and endangered mollusks (naiads) of the U.S.A. Department of the Interior, Fish and Wildlife Service.

243. Stansbery. D.H. 1972. A preliminary list of the naiad shells recovered from the Buffalo site. Appendix A (105-106. In: Brogles. B.J. (Ed.), A late I7th Century Indian Village Site (46 Pu 3I) in Putnam County, West Virginia. Report of Archaeological Investigations No. 5 , West Virginia Geologial and Economic Survey, Morgantown, West Virginia.

244. Stansbery, D.H. 1973. A preliminary report on the naiad fauna of the Clinch River in the Southern Appalachian mountsins of Virginia and Tennessee (Mollusca : Bivalvia : Unionoida). Bulletin of the American Malacological Union 38th Annual Meeting, 1972: 20-22.

245. Stansbery, D.H. 1976. Naiad Mollusks. In: Boschung, H. (Ed.), Endangered and threatened plants and animals of Alabama. Bulletin of the Alabama Museum of Natural History 2: 42-52.

246. Stansbery, D.H. 1976. The occurrence of endangered species of naiad mollusks in Lower Allum and Big Walnut Creeks. Report to Ohio Department of Transportation. OSUMZ Report 17.

247. Stansbery, D.H. 1976. Quadrula sparsa (Lea 1841). In: Status of endangered fluviatile mollusks in centrol North America. U.S. Department of the Interior, Fish and Wildlife Service, Bureau of Sports Fisheries and Wildlife. Washington, D.C. Contract No. 14-16-0008-755.6 pp.

248. Stanabery, D.H. 1976. Taxolasma cylindrellus (Lea 1868). In: Status of endangered fuviatile mollusks in central North America. U.S. Department of the Interior, Fish and Wildife Service, Bureau of Sports Fisheries and Wildlife. Washington, D.C. Contract No. 14-16-0008-755.8 pp.

249. Stansbery, D.H. 1979. The Stotus of Lemiox rimosus (Rafinesque, 1831) (Mallusca:Bivalvia:Unionoido). Report for The Office of Endangered Species, Fish and Wildlife Service, U.S. Department of the Interior. 
250. Stephanides, T. 1948. A survey of the freshwater biology of Corfu and of other regions of Greece. Prakrika tou Ellenikou 'Udrobialogikou Insritoutau, Athenai 2: 156.

251. Strayer, D. 1980. The freshwater mussels (Bivalvia: Unionidae) of the Clinton River, Michigan with comments on man's impact on the fauna, 1870-1978. Nautilus 94(4): 142-149.

252. Suomalainen, E.; Kaisila, J. and Mikkola, K. 1980. Noteworthy recorda of Finnish Lepidoptera 1955-1974. 1. Hesperioider, Papilionoidea, Bombycoidea and Geometroidea. Notulae Entomalagicae 60: 49-61.

253. Talbot, G. 1939. The Founa of British India, including Ceylon and Buma. Butterflies Vol. 1. Taylor and Francis Lid., London.

254. Tenneasee Valley Authority 1978. Virginis Mollugk Survey. Contract Report. July 1, 1977 - June 30, 1978. Division of Forestry, Fisheries, and Wildlife Development. Norris, Tennessee 3728 .

255. Trukada, E. and Nishiyama, J. 1982. Butterflies of the South East Asian Islands. Vol.l Papilionidae. (transl. K. Morishils) Plapac Co. Lid., Tokyo. 457 pp.

256. Turgeon, D.D., Bogan, A.E., Coan, E.V., Emerson, W.K. Lyons, W.G., Pratt, W.L., Roper, C.F.E., Scheltema, A., Thompson, F.G. and Williams, J.D. 1988. Common and scientific names af aquatic invertebrates from the United States and Canada: mollusks. American Fisheries Society Special Publication 16. Bethesda, Maryland.

257. Turner, T.W. 1983. The status of the Papilionidae, Lepidoptera of Jamaics with evidence to support the need for conservation of Papilia hamerus Fabricius and Euryrides marcellinus Doubleday. Unpublished report. 14 PP.

258. Tvermyr, S. 1965. Legeiglen (Hiruda medicinalis L.) finnes unns frittevende i Aust-Agder. Fauna, Oslo 18: 136-139.

259. UNEP/IUCN 1988. Coral Reefs af the World. Volume I: Atantic and Eastem Pacific. UNEP Regional Sess Directories and Bibliographies. JUCN, Gland, Switzerland and Cambridge, U.K./UNEP, Nairobi, Kenya .

260. UNEP/IUCN 1988. Coral Reefs of the Warld. Volume 2: Indian Ocean, Red Sea and Gulf. UNEP Regional Seas Directories and Bibliographies. IUCN, Gland, Switzerland and Cambridge, U.K./UNEP, Nairobi, Kenya.

261. UNEP/IUCN 1988. Caral Reefs of the World. Volume 3: Central and Westem Pacific. UNEP Regional Seas Directories and Bibliographies. IUCN, Gland, Swizerland and Cambridge, U.K./UNEP, Nairobi, Kenya.

262. U.S. Departmemt of the Interior 1981. Endangered and threatened wildlife and plants; listing the Hawaiian (Oahu) tree snails of the genus Achatinella as Endangered Species. Federal Register 46(8): 3178-3182.

263. U.S. Department of the Interior 1984. Recovery plans approved for five mollusks. Endangered Species Technical Bullerin 9(1): 7-12.

264. U.S. Fish and Wildlife Service 1976. Endangered status for 159 taxa of animals. Federal Register 41(115): 24062-24067.

265. U.S. Fish and Wildlife Service 1977. Endangered and threatened wildlife and plants. Determination that the Tan Riffle Shell is an Endangered Species. Federal Register 42(163): 42351-42353.
266. U.S. Figh and Wildlife Service 1984. Endangered and threatened wildlife and plants; Removal of Epioblasma (= Dysnomia) sampsoni, Sampson's Pearly Mussel, from the list of Endangered and Threatened Wildlife. Federal Register 49(5): 1057-1058.

267. Usher, G. and Salm, R.V. 1984. From filmstsr to floor tile. Voice of Nature 20: 12-13

268. Usher, G.F. 1984. Coral reef inveruebrates in Indonesia: their exploitation and conservation needs. IUCN/WWF Report No. 2, Bogor, Indonesia, 100 pp.

269. de Viedma, M.G. and Gomez-Bustillo, M.R. 1976. Libro Rojo de Los Lepidopteros Ibericas. Publicacione del Ministerio de Agricultura Secretsria General Tecnica, Madrid. 120 pp

270. Walker, K.J. 1982. A survey of the distribution and density of Pawelliphanta annectens in North-west Nelson. New Zealand Wildlife Service, Department of Internal Affairs, Wellington. Fauna Survey Unit Repon 31: 23 pp.

271. Walker, K.J. 1982. Distribution and status of Pawelliphanta land snails in the Mokihinui State Forest, and recommendations for conservation reserves. New Zealand Wildlife Service. Wellington. Fauna Survey Unit Repart 34: 10 Pp.

272. Wankowski J.W.J. 1979. Report on a preliminary survey of Nuguria, Nukumann and Takuu Atolls. Mimeo Reports, Research and Surveys Branch, DPl Fisheries, P.N.G. 27 Pp.

273. Welch, D'alte A. 1938. Distribution and variation of Achatinella mustelina Mighels, in the Waianae Mountains, Oahu. Bemice P. Bishop Misseum Bulletin. Honolulu 152: 164 pp.

274. Welch, D'alte A. 1942. Distribution and variation of the Hawaiian tree snail Achotinella opexfulva Dixon in the Koolau Range, Oahu. Smithsonian Miscellaneous Collecrions 103(1): 1-236

275. Welch, D'alte A. 1954. Distribution and variation of the Hawaiian tree gnail Achatinella bulimoides Swainson on the leeward and northern slopes of the Koolau Range, Oahu. Proceedings of the Academy of Natural Sciences of Philadelphia 106: 63-107.

276. Welch, D'alte A. 1958. Distribution and variation of the Hawaiian tree snail Achatinella bulimaides Swainson on the windward slope of the Koolau Range, Oahu. Praceedings of the Academy of Natural Sciences of Philadelphia 110: 123-212.

277. Wells, S. and Coombes, W. 1987. The status and trade in the Medicinal Leech. Traffic Bullerin 8(4): 64-69.

278. Wells, S.M. 1981. Giant clams - a case for CITES listing. Traffic Bulletin 3(6): 60-64.

279. Wells, S.M., Pyle, R.M. and Collins, N.M. 1983. The IUCN Invertebrate Red Data Book. IUCN, Cambridge and Gland 632 pp.

280. Wells, S.M., Elliott, J.M. and Tullett, P.A. 1984. Status of the Medicinal leech Hiruda medicinalis. Letter to the Editor. Bialagical Conservation 30: 379-380.

281. Whitlock, M.R., O'Hare, P.M., Sandera, R. and Morrow, N.C. 1983. The medicinal leech and its use in plastic surgery: a possible case for infection. British Joumal of Plastic Surgery 36: 240-244. 
282. Woodhouse, L.G.O. 1950. The Butterfly Fauno of Ceylon. Second Edition. Colombo Apothecaries' Co. Lid., Colombo. 231 pp.

283. Yamaguchi, M. 1977. Conservation and cultivation of giant clams in the tropical Pacific. Biological Conservation 11: 13-20.
284. Zapkuvene, D.V. 1972. Breeding and growing of medicinal leeches under laboratory conditions. 1. Breeding of Hirudo medicinalis $\mathrm{f}$. serpentina and $\boldsymbol{H}$. medicinalis $\mathrm{f}$. officinalis. Lietuvos TSR Mokseu Akademijos Darbai Serija C. Trudy Akademii Nauk Lisovskoi CCP, Ser. B) 3(59): 71-84. 



\section{Class: ANTHOZOA}

\section{Order ANTIPATHARIA}

\section{Family ANTIPATHIDAE}

Antipathes Pallas 1766 (approximately 93-96 species) II CT $58,59,89$

(Reported from all oceans [25,27]: Atlantic from north to south [26,59], including the Caribbean $[34,58,59]$, Gulf of Mexico [58] and Mediterranean [26,58,59]; Indo-Pacific [18,34,57,59,60,98], including Red Sea [57,59] and Arabian Gulf [18])

Anguilla [59]; Australia [101]; Azores [26,80]; Bahamas [58,59]; Barbados [4,50,58,59]; Brazil [58,59]; British Indian Ocean Territory: Chagos [18]; Canary lslands [80]; Cape Verdes [80]; Chile: including Juan Fernandez [4,58]; China [101]; Colombia [59,98]; Cuba [58]; Dominica [4,58,59]; Dominicar Republic [59]; Ecuador [53,98]; Fiji [4,34]; France [4,101]; Galapagos 1slands [53,54,60,79,98]; Gibraltar [26,59]; Grenada [58]; Guadeloupe $[4,11,58,59]$; Guam [98]; Guyana [58]; Hawaiian Islands $[33,34,35,98,101]$; Honduras $[58,59]$; Hong Kong [101]; India: includiing Andaman Islands [4,18]; Indonesia [4,18,38,39,72,84,101]; Italy $[1,4,29,44,59,101]$; Jamaica [56,58,59,97]; Japan [4,57,88,98]; Korea [90]; Liberia [4]; Madagascar [4,38]; Madeira [4,26,40,59,80]; Maldives [18]; Martinique [4,12,58]; Mauritius: including Cargados Carajos, Rodrigues [4,16,18]; Mexico [7,41,59]; Montserrat [4,58]; Morocco [26,80,101]; Netherlands Antilles [98]; New Zealand [4,21,22,23,59,94,98]; Palau [34]; Panama [4,58,59,60,95,98]; Philippines [4,18,34,72,101]; Portugal [26]; Puerto Rico [58]; Réunion [16]; Saint Helena [4,59]; Saint Lucia [4,59]; Saint Vincent [4,58]; Saudi Arabia [4,59]; Seychelles: including Amirantes [18]; Sri Lanka [4,18,93]; Suriname [58,59]; Taiwan [4,18,98]; Tonga [34]; Trinidad [58,97]; USA [4,8,58,59]; Vanuatu [4]; Virgin Islands of the United States $[12,58,59,65]$; Venezuela $[58,59]$

Antipathes abies (Linnaeus 1758)

(Indo-Pacific [18])

India: Andaman Islands [4,18]; Indonesia: Moluccas [39]; Madagascar [38]; Mauritius: including Cargados Carajos, Rodrigues [16,18]; Mozambique [91]; Philippines [18]; Réunion [16]; Seychelles: including Amirantes [18]; Sri Lanka [18,93]; Taiwan [18]

Antipathes aculeata Brook 1889

Indonesia: Aru Islands [4]

Antipathes alata (Brook 1889)

Mauritius [4]

Antipathes americana Duchassaing \& Michelotti 1860

Known only from the Lesser Antilles and north-west Gulf of Mexico [58]

Grenada [58]; Venezuela [58]; Virgin Islands of the United States: Saint Thomas $[4,12,58]$;

Antipathes aperta Totton 1923

New Zealand $[21,22,23,59,98]$

Antipathes arborea Dana 1846

Fiji $[4,30]$

Antipathes assimilis (Brook 1889)

Antipathes atlantica Gray 1857

Jamaica [97]; Trinidad [97] 
Antipathes barbadensis (Brook 1889)

Barbados [4]; Trinidad [97]

Antipathes bifaria Brook 1889

Japan [88]; Taiwan [4]

Antipathes boscii Lamouroux ?

USA [4]

Antipathes catharinae Pax 1932

Antipathes ceylonensis Thomson \& Simpson 1905

Seychelles: including Amirantes [18], Sri Lanka [18,93]

Antipathes chamaemorus Pax 1932

Japan

Antipathes chota Forster Cooper 1903

Maldives [17,18]

Antipathes columnaris (Duchassaing 1870)

(Throughout the Caribbean but not Gulf of Mexico [59])

Anguilla [59]; Bahamas [59]; Barbados [4,78]; Brazi] [59]; Dominica [4,78]; Dominican Repub]ic [59]; Grenadines [4]; Guadeloupe [4,11,59,78]; Martinique [4,78]; Mexico [59]; Saint Lucia [4,59,78]; Saint Vincent [4,59,78]; Suriname [59]; Venezuela: Aves Island [59]; Virgin Islands $[4,59,78]$

Antipathes contorta (Brook 1889)

Antipathes crispa (Brook 1889)

Antipathes cupressus Pallas 1766

Antipathes curvata van Pesch 1914

Indonesia: Irian Jaya [72]

Antipathes cylindrica Brook 1889

Antipathes delicatula Schultze 1896

Indonesia: Ternate [84]

Antipathes densa Silberfeld 1909

Japan $[87,88]$, Taiwan

Antipathes dichotoma Pallas 1766

(Indo-West Pacific [34] and eastern Atlantic [26], including the Mediterranean [59, 101], Bay of Biscay [24] and Josephine Seamount [26])

Australia [101]; China [101]; Fiji [34]; France [4,101]; Guam [98]; Hawaiian lslands [34,98]; Hong Kong [101]; India [101]; Indonesia [101]; Italy [4,29,44,101]; Mauritius: including Rodrigues [16]; Mexico [7]; Netherlands Antilles: Bonaire [98]; Palau [34]; Philippines [101]; Réunion [16]; Tonga [34]

Antipaihes dubia (Brook 1889)

Japan [4]

Antipathes elegans (Brook 1889) 
Antipathes ericoides Pallas 1766

(Indo-Pacific [4,59] including the Red Sea [57])

Indonesia: Banda [72], Moluccas [39], Roti [72]; Madagascar [38]

Antipathes erinaceus (Roule 1905)

Antipathes eupteridea Lamouroux, Bory de Saint Vincent \& Deslongchamps 1824

Martinique $[4,12,59,78]$

Antipathes fernandezi Pourtalès 1874

Chile: Juan Fernandez $[4,58]$

Antipathes fiordensis Grange 1990

New Zealand [23]

Antipathes flabellum Pallas 1766

(Indo-Pacific [59])

Indonesia: Ternate [84]; Madagascar [4]

Antipathes fragilis (Brook 1889)

Italy [29]

Antipathes fruticosa Gray 1857

New Zealand: Stephens Island [4]

Antipathes furcata Gray 1857

(Eastern and western Atlantic; throughout the Caribbean [59])

Bahamas [59]; Barbados [59]; Madeira [4,40,59]

Antipathes galapagensis Deichmann 1941

Galapagos Islands $[53,79,98]$

Antipathes gallensis Thomson \& Simpson 1905

Sri Lanka [93]

Antipathes glutinata Totton 1923

New Zealand [94]

Antipathes grandiflora Silberfeld 1909

Japan $[87,88]$

Antipathes grandis Verrill 1928

China [101]; Hawaiian Islands [33,34,98,101]; Hong Kong [101]; Mexico [7]

Antipathes grayi (Roule 1902)

Azores [80]; Canary Islands [80]; Morocco

Antipathes hirta Gray 1857

(Throughout the Caribbean [58])

Barbados [4,58,78]; Grenada [4,58,78]; Guyana [58]; Jamaica [58]; Martinique [58]; Puerto Rico [58]; Saint Vincent [58]; Trinidad [97]; USA [58]; Venezuela [58]

Antipathes hypnoides (Brook 1889)

Mauritius [4]

Antipathes indistincta van Pesch 1914

Indonesia: Damar [72] 
Antipathes intermedia (Brook 1889)

Hawaiian Islands [35]; Japan [4,57,98]; Taiwan [98]; USA [8]

Antipathes lata Silberfeld 1909

(Southern part of the Yellow Sea and western part of the Sea of Japan [90])

Japan [87,88]; Korea [90]

Antipathes lenta Pourtalès 1871

(Gulf of Mexico, east coast of central and south America and Mediterranean Sea [58])

Barbados [58]; Colombia [58]; Cuba [58]; Honduras [58]; Panama [58]; Saint Vincent [58]; Trinidad [58]; USA [58]; Venezuela [58]

Antipashes lentipinna Brook 1889

(Indo-Pacific including Red Sea [59])

Mozambique [91]; Saudi Arabia [4,59]

Antipathes longibrachiata van Pesch 1914

Indonesia: Lombok, Moluccas [39]; Japan [88]; Madagascar [38]

Antipathes mediterranea Brook 1889

(Known only from Gulf of Naples, Mediterranean [4,59])

Antipathes minor (Brook 1889)

Chile [4]

Antipathes myriophylla Pallas 1766

(Indo-Pacific $[18,59]$ )

Indonesia [18,30,39]; Madagascar [38]; Mauritius: including Rodrigues [16]; Philippines [4,18,30,72]; Réunion [16]

Antipathes nilanduensis Forster Cooper 1903

Maldives [17]

Antipathes panamensis Verrill 1869

Colombia [98]; Ecuador [53,98]; Galapagos 1slands [54,60,98]; Panama $[4,59,60,95,98]$

Antipathes paniculata Esper 1797

Mauritius [4]; Philippines [4]; Sri Lanka [4]

Antipathes pauroclema Pax 1932

Antipathes pectinata Lamarck 1816

Antipathes pedata Gray 1857

(Caribbean and Yucatan Peninsula [4,59])

Mexico; Panama [59]; Suriname [59]

Antipathes pennacea Pallas 1766

(Indo-Pacific, central and south Atlantic, throughout Caribbean [59])

Bahamas [59]; Barbados [50,59]; Dominica [59]; Honduras [59]; Indonesia: Flores, Kalimantan, Roti [72]; Jamaica [56,59]; Martinique [59]; Mexico [59]; Netherlands Antilles [98]; Panama [59]; Philippines [72]; Saint Helena [4,59]; Trinidad [97]; USA [59]; Virgin Islands of the United States [59]: Saint Thomas [13]

British Indian Ocean Territory: Chagos [18]; Indonesia: Solor 
CITES RL Ref

Antipathes plantagenista (Forster Cooper 1903)

Maldives [17]

Antipathes pluma Gray 1857

Antipathes pseudodichotoma Silberfeld 1909

Japan [88]

Antipathes punctata Roule 1905

Hawaiian lslands [35]

Antipathes reticulata Esper 1797

Mauritius: including Rodrigues [16]; Philippines [4]; Réunion [16]

Antipathes rhipidion Pax 1916

Virgin 1slands of the United States: Saint Thomas [65]

Antipathes rigida Pourtalès 1880

Bahamas [58,78]; Barbados [58]; Colombia [58]; Guadeloupe [4]; Venezuela [58]

Antipathes robillardi Bell 1891

Mauritius [2]

Antipathes rugosa (Thomson \& Simpson 1905)

Sri Lanka [93]

Antipathes salicoides Summers 1910

Mozambique [91]

Antipathes salix Pourtalès 1880

(Caribbean [34])

Guadeloupe $[58,78]$

Antipathes sarothamnoides (Brook 1889)

Vanuatu [4]

Antipathes sarothrum Pax 1932

Antipathes sealarki Forster Cooper 1909

(?Indian Ocean [18])

Antipathes sibogae van Pesch 1914

Indonesia: Kalimantan [72]

Antipathes simplex (Schultze 1896)

Indonesia: Temate [84]

Antipathes speciosa (Brook 1889)

Chile [4]

Antipathes spinescens Gray 1857

Liberia [4]

?Antipathes spinosa (Carter 1880)

Described as very similar to and possibly a synonym of Antipathes panamensis [60]

Maldives [17]; Sri Lanka [4] 
Antipathes squamosa W. Koch 1886

(?Rolas (Gulf of Guinea) [4])

Antipathes stechowi (Pax 1932)

Antipathes strigosa (Brook 1889)

New Zealand [4]

Antipathes subpinnata Ellis and Solander 1786

(Mediterranean, Bay of Biscay [26], Great Meteor and Josephine Seamounts [26])

Gibraltar [59]; Italy [1,29,44]; Madeira [30,59]; Portugal [26]

Antipathes tanacetum Pourtalès 1880

(Throughout Caribbean [58])

Bahamas [58]; Brazil [58]; Colombia [58]; Dominica [4,58,78]; Grenada [4,78]; Grenadines [4]; Martinique [4,58,78]; Montserrat [4,58,78]; Saint Vincent [58]; Suriname [58]; USA [58]; Venezuela [58]

?Antipathes tenuispina (Silberfeld 1909)

Indonesia: Timor [72]; Japan [87,88]

?Antipathes ternatensis Schultze 1896

Indonesia: Ternate [84]

Antipathes thamnea Warner 1981

Trinidad [97]

Antipathes thamnoides Schultze 1896

Indonesia: Ternate [4]

Antipathes tristis (Duchassaing 1870)

Barbados [4,78]; Guadeloupe [4,11]; Martinique [4,78]; Montserrat [4,78]; Mozambique [91]; Saint Lucia [4,78]

Antipathes ulex Ellis \& Solander 1786

Hawaiian Islands [35]; Indonesia: Java [4], Solor [72]; Philippines [4]

Indonesia: Solor [72]

?Antipathes valdiviae $\operatorname{Pax} 1915$

India: Nicobar Islands [64]

Antipathes vericillata (Brook 1889)

Mauritius [4]

Antipathes viminalis Roule 1902

China [101]; Hong Kong [101]; Morocco $[80,101]$

Antipathes virgata Esper 1797

(Indian Ocean, including Arabian Gulf [4, 18,59]; Mediterranean [48])

Azores [80]; Cape Verdes [80]; Madeira [80]; Mauritius: Cargados Carajos [18]; Philippines [18]

Antipathes wollastoni Gray 1857

Madeira [4]

Antipathes zoothallus $\operatorname{Pax} 1932$ 


\section{Aphanipathes Brook 1889 (approximately 9 species) $\quad 11 \quad$ CT $\quad 58,59,89$}

(Reported from the Caribbean [58], Gulf of Mexico [58], south-east Atlantic [26] and the Indian Ocean [4])

Bahamas [58]; Barbados [4,58]; British Indian Ocean Territory: Chagos [18]; Cuba [4,58]; Dominica [4];

Grenada [4,58]; Guadeloupe [4,58]; Indonesia [4,72]; Martinique [4,58]; Mexico [58]; Montserrat [4,58];

Nicaragua [58]; Saint Vincent: including Grenadines [4,58]; Sri Lanka [18]; USA [58]

Aphanipathes abietina (Pourtalès 1874)

(Throughout the Caribbean and northern Gulf of Mexico [58]; Grand Meteor Seamount [26])

Bahamas [58]; Barbados [4,58]; Martinique [58]; Mexico [58]; Nicaragua [58]; Saint Vincent: Grenadines [58]

Aphanipathes cancellata Brook 1889

Indonesia: Irian Jaya [72], Kai Islands [4]

Aphanipathes filix (Pourtalès 1867)

Bahamas [58]; Barbados [4,58,78]; Cuba [4,58,73]; Dominica [4,78]; Guadeloupe [4,58,78]; Martinique [4,58];

Montserrat [4,78]; Saint Vincent: including Grenadines [4,58,78]; USA [58]

Aphanipathes hancocki Forster Cooper 1909

British Indian Ocean Territory: Chagos [18]

Aphanipathes humilis (Pourtalès 1867)

Bahamas [58]; Barbados [4,58,78]; Cuba [4,58,73]; Grenada [4,58,78]; Mexico [58]; Montserrat [4,58,78];

Saint Vincent $[4,78]$; USA [58]

Aphanipathes reticulata van Pesch 1914

Indonesia: Roti [72]

Aphanipathes somervillei Forster Cooper 1909

British Indian Ocean Territory: Chagos [18]

Aphanipathes thyoides (Pourtalès 1880)

(Gulf of Mexico and Caribbean [58])

Cuba [58]; Saint Vincent $[4,58,78]$

Aphanipathes undulata (van Pesch 1914)

(Indo-Pacific [98])

Bathypathes Brook 1889 (approximately 12 species) II $\quad$ CT $\quad 59,63,89$

Reported from all oceans [25,27,59], including the Antarctic [40,59,63,92]; Atlantic [59,63,64], including Caribbean [59] and western shores of Europe [63]; Indian Ocean [18,63], including the Arabian Sea [63] and Bay of Bengal [63]; north to south Pacific, including Tasman Sea [63]

Australia [4]; Azores [29a]; Cocos (Keeling) Islands [63]; Cuba [59]; Falkland Islands [63]; Hawaiian Islands [4,63]; India [18,37,62c, 72]; Indonesia [4,62c,63]; Mexico [59]; Morocco [63]; Mozambique [63]; New Zealand [94]; Nicobar Islands [62c]; Papua New Guinea [4]; Russia [18]; Saint Kitts-Nevis: Nevis lsland [59]; Puerto Rico [59]; Seychelles [63]; Sri Lanka [18,62c,72]; USA [59]

Bathypathes alternata Brook 1889

Hawaiian Islands [4]

Bathypathes bifida Thomson 1905

Antarctic (7]'22'S 1634'W) [92]

Bathypathes erotema Schultze 1903

Antarctic (63\%16'S 57 $\left.51^{\prime} \mathrm{E}\right)$ [40]

Bathypathes euantha Pasternak 1958 
Bathypathes galatheae Pasternak 1977

(Gulf of Panama [63])

Bathypathes heterorhodzos (Forster Cooper 1909)

Probably cosmopolitan [59]

Cuba [59]; Russia (74. $\left.4^{\prime} \mathrm{N} 79^{\circ} 32^{\prime} \mathrm{E}\right)$

Bathypathes lyra Brook 1889

(All oceans [25], central Coral Sea [4], southem Arabian Sea, Bay of Bengal; Atlantic Ocean [63], including Bay of Biscay [24])

Azores [29a]; Hawaiian 1slands [63]; India: Laccadives [62c], Nicobar Islands; Indonesia: Sumatra [62c]; Seychelles [63]; Sri Lanka [62c]

\section{Bathypathes patula Brook 1889}

(All oceans [25,59], coast of east and south east Africa, Makassar Strait, Tasman Sea, Kermadec Trench, central east Pacific, Arabian Sea, Bay of Bengal, Java trench, western shores of Europe, Antarctic [63], Mid-Atlantic Ridge [64], Bay of Biscay [24]) Cocos (Keeling) Islands [63]; Falkland 1slands [63]; French Southern and Antarctic Territories: Amsterdam 1sland [27]; India [18,72]; Indonesia: Banda [4]; Mexico [59]; Morocco [63,80]; Mozambique [63]; Papua New Guinea [4]; Saint Kitts and Nevis: Nevis Island [63]; Puerto Rico [59]; Sri Lanka [18,72]; USA [59]

Bathypathes platycaulus Totton 1923

New Zealand [94]

Bathypathes quadribrachiata van Pesch 1914

Indonesia: Banda

Bathypathes scoparia Totton 1923

New Zealand

Bathypathes tenuis Brook 1889

Australia: South Australian Basin [4]

Cirrhipathes Blainville 1834 (approximately 13 species)

$11 \quad \mathrm{CT} \quad 89$

(Reported from the Caribbean [98] and Indo-Pacific [98])

Barbados [50]; China [100,101]; Fiji [4]; Hawaiian Islands [35]; Hong Kong [101]; Indonesia [4,39,72,101]; Iran [101]; Jamaica [99]; Japan [88]; Korea [90,97]; Maldives [18]; Mauritius: including Rodrigues [16]; Philippines [34]; Réunion [16]; Seychelles [18]; South Africa [101]; Sri Lanka [4,18,101]; Trinidad [97]

Cirrhipathes aggregata (van Pesch 1914)

Indonesia: Salayar [72]

\section{Cirrhipathes anguina Dana 1846}

(Indo-Pacific [59,98], including Red Sea [4, 18])

Fiji [4]; Hawaiian Islands [35]; Indonesia: including Belitung, Moluccas [4,39]; Korea [90]; Maldives [17,18]; Mauritius: including Rodrigues [16]; Mozambique [91]; Réunion [16]; Seychelles [18]; Sri Lanka [4,18], Taiwan

Cirrhipathes contorta van Pesch 1910

Indonesia: Banda [72]

Cirrhipathes musculosa van Pesch 1910

China [101]; Hong Kong [101]; Indonesia: Sumbawa [101]

Cirrhipathes nana van Pesch 1910

Indonesia: Salebabu (Talaud) [72] 
Cirrhipathes rumphii van Pesch 1910

(Indo-Pacific [59], including east African coast [101])

China [101]; Hong Kong [101]; Indonesia: Komodo, Roti, Solor [72]; Iran [101]; South Africa [101]; Sri Lanka [101]

Cirrhipathes saccula (van Pesch 1914)

(South China Sea [100])

China [100]; Indonesia: Kai Islands [72]

Cirrhipathes semiglabra van Pesch 1914

Cirrhipathes sinensis Zou \& Zhao 1984

China [101]; Hong Kong [101]

Cirrhipathes solorensis (van Pesch 1914)

Indonesia: Solor [72]

Cirrhipathes spiralis (Linnaeus 1758)

(Indo-Pacific [59,99])

Barbados [78]; Cuba [78]; Grenada [78]; Hawaiian 1slands [35]; Indonesia: Aru Islands [72], Moluccas [4, 14], Solor, Sumbawa, Waigeu [72]; Japan [88]; Maldives [17]; Martinique [78]; Mauritius: including Rodrigues [16]; Montserrat [78]; Mozambique [91]; Réunion [16]; Saint Vincent [78]; Sri Lanka [4,17], Taiwan

Cirrhipathes translucens van Pesch 1910

Indonesia: Aru Islands [72]

Cirrhipathes variabilis van Pesch 1914

Cladopathes Brook 1889 (1 species)

Cladopathes plumosa Brook 1889

South Africa: Prince Edward Island [4]

Hexapathes Kinoshita 1910 (1 species)

II

CT $\quad 59$

(North-west Pacific [59])

Hexapathes heterosticha Kinoshita 1910

Japan [42]

Hillopathes van Pesch 1914 (1 species)

Hillopathes ramosa (van Pesch 1910)

Leiopathes (Gray 1842) (approximately 3 species)

II $\quad$ CT 59

(Reported from the Atlantic [28], including the Bay of Biscay [28], Gulf of Mexico [59] and Mediterranean $[28,59])$

Azores [28]; Bahamas [59]; Cape Verde [80]; French Southern and Antarctic Territories: Saint Paul Island [36];

Hawaiian Islands [35]; Italy [29,44]; Madeira [28,40,59,80]; Morocco [28,80]; USA [28,59]

Leiopathes expansa Johnson 1900

Madeira [40]

Leiopathes glaberrima (Esper 1794)

(North Atlantic, including Mediterranean, Bay of Biscay and Great Meteor Seamount; Gulf of Mexico [28,59])

Azores [28]; Bahamas [59]; French Southem and Antarctic Territories: Saint Paul [27]; Italy [29,44]; Madeira [28,40,59]; Morocco [28]; Spain [28]; USA [28,59] 
Leiopathes grimaldii Roule 1902

Cape Verde [80]; Madeira [80]; Morocco [80]

Parantipathes Brook 1889 (approximately 5-7 species) II CT $59,63,89$

(Reported from all oceans [27]: Atlantic [25,26,64], including Caribbean [58], Bay of Biscay [25], Mediterranean [59]; Indo-Pacific [63,90])

Cape Verde [80]; Faeroe Islands; France; Guyana [58]; Indonesia [63,72]; Italy [4,29,44]; Korea [90]; Malaysia [63]; Martinique [4,12]; Mexico [58]; Morocco [28,80]; New Zealand [94]; Philippines [72]; Puerto Rico [58];

Saint Lucia [58]; USA [4,58]

Parantipathes laricides van Pesch 1914

Indonesia: Seram Sea (3³7'S 131²6’E) [72]

Parantipathes larix (Esper 1794)

(Atlantic [25,26,64], including Mediterranean [4,59])

Cape Verde [80]; Faeroe Islands; France [37]; Italy [4,29,44]; Martinique [4, 12,13]; Morocco [80]; Philippines [72]

Parantipathes lilliei (Totton 1923)

New Zealand [94]

?Parantipathes strigosa (Brook 1889)

Parantipathes tetrasticha (Pourtalès 1868)

Guyana [58]; Mexico [58]; Puerto Rico [58]; Saint Lucia [58]; USA [4,58,74]

Parantipathes tristicha van Pesch 1914

Indonesia: Seram [72]

?Parantipathes wolffi Pasternak 1977

Strait of Malacca [63]

Schizopathes Brook 1889 (approximately 3 species) II $\quad$ CT $\quad 59,89$

(Reported from northern Indian Ocean [18], south Atlantic [18], and northern Pacific: Hawaiian Islands [35])

France; Hawaiian Islands [35]; Indonesia [4]; Papua New Guinea [4]; South Africa: Prince Edward Island [4];

Unuguay [4]

Schizopathes affinis Brook 1889

(North Indian Ocean; south AtJantic [18])

France; Indonesia: Banda [4]; Papua New Guinea [4]

Schizopathes conferta Brook 1889

Hawaiian 1slands [35]; South Africa: Prince Edward Island [4]

Schizopathes crassa Brook 1889

France [37]; Uruguay [4]

Sibopathes van Pesch 1914 (I species)

II $\quad$ CT $\quad 59,89$

Sibopathes gephura van Pesch 1914

Indonesia: Timor [72] 
Stichopathes Brook 1889 (approximately 19 species) $11 \quad$ CT

(Atlantic [25,28], including Bay of Biscay [24]; Indo-Pacific [18,25,100], including north-east Pacific [61] and South China Sea [100])

Australia [4,32]; Azores [25,29a]; Barbados [50]; British Indian Ocean Territory: Chagos [18]; China [100]; Djibouti [46]; Hawaiian 1slands [35]; India: Andaman 1slands [18]; Indonesia [63,72]; Japan [63,88]; Madagascar [38]; Madeira [4,25,40,80]; Mauritius [4,18]; Morocco [25]; Netherlands Antilles [9]; Philippines [72]; Saint Helena [4]; Seychelles [18]; Sri Lanka [18,93]; Trinidad [97]; USA [19]

Stichopathes abyssicola Roule 1902

(Atlantic [28], including Bay of Biscay [24]; South China Sea [100])

Azores [25]; China [100]; Madeira [25,80]; Morocco [25,28]

Stichopathes alcocki Forster Cooper 1909

Sri Lanka [18]

Stichopathes bournei Forster Cooper 1909

(North Indian Ocean [18], South China Sea [100])

China [100]

Stichopathes ceylonensis Thomson \& Simpson 1905

(South China Sea [100])

China [100]; Indonesia: Kai Islands [72]; Sri Lanka [93]

Stichopathes contorta Thomson \& Simpson 1905

(South China Sea [100])

China [100]; Sri Lanka [93]

Stichopathes desbonni (Duchassaing \& Michelotti 1864)

(South China Sea [100])

China [100], Guadeloupe [13]; Montserrat [78]

Stichopathes echinulata Brook 1889

(Indian Ocean [18])

Djibouti [46]; Hawaiian Islands [35]; Indonesia: Moluccas [39]; Madagascar [38]; Mauritius [4, 18]; Mozambique [91]; Seychelles [18]; Sri Lanka [93]

Stichopathes filiformis (Gray 1868)

(South China Sea [100])

Australia [4,32]; China [100]; Japan [88]; Madeira [80]; Saint Helena [4]

Stichopathes flagellum (Brook 1889)

(South China Sea [100])

China [100]; Madeira [80]; Mozambique [91]

Stichopathes gracilis (Gray 1857)

(Indo-Pacific, Atlantic [25], including Bay of Biscay [24])

Azores [29a]; Indonesia: Flores [72]; Jamaica [97]; Madeira [4,40]; Mozambique [91]; Netherlands Antilles [9];

Seychelles: Amirantes [18]; Sri Lanka [93]

Stichopathes longispina Forster Cooper 1909

Seychelles [18]

Stichopathes lutkeni Brook 1889

(Northern Indian Ocean; Atlantic [18]; West Indies [4,98])

Barbados [50]; Trinidad [97] 
Stichopathes papillosa Thomson \& Simpson 1905

India: Andaman Islands [18]; Sri Lanka [18,93]

Stichopathes paucispina (Brook 1889)

(Eastern north Pacific [61])

Opal Seamount (30³0'N $\left.121^{\circ} 54^{\prime} \mathrm{W}\right)$; Philippines [72]

Stichopathes regularis Forster Cooper 1909

British Indian Ccean Territory: Chagos [18]; Maldives [17]; Sri Lanka [18]

Stichopathes semiglabra van Pesch 1914

(South China Sea [100])

China [100]; Indonesia: Sulawesi [72]

Stichopathes seychellensis Forster Cooper 1909

Seychelles [18]

Stichopathes spiessi Opresko \& Genin 1990

(Eastern north Pacific [61])

Fieberling Seamount $\left(32^{\circ} 26^{\prime} \mathrm{N} 127^{\circ} 47^{\prime} \mathrm{W}\right)$; Jasper Seamount $\left(30^{\circ} 26^{\prime} \mathrm{N} 122^{\circ} 43^{\prime} \mathrm{W}\right)$

Stichopathes variabilis (van Pesch 1914)

Indonesia [63,72]; Japan [63]

Taxipathes Brook 1889 (I species)

II

CT

Taxipathes recta Brook 1889

Saint Helena: Ascension lsland [4]

Tropidopathes Silberfeld 1909 (1 species)

II

CT

Tropidopathes saliciformis Silberfeld 1909

Japan $[87,88]$ 


\section{REFERENCES - BLACK CORALS}

1. Aiello, A., Fattorusso, E. and Menna, M. 1992. 4 new bioactive polyhydroxylated sterols from the black coral Antipathes subpinnota. J. Noiural Products - Lloydia 55: 321-325.

2. Belt, F.J. 1891. Contributions to our knowledge of the Antipatharian corals. II. On a remarkable Antipathid from the neighbourhood of Mauritius. Trans. Zool. Soc. 13: 9192.

3. Blainville, H.M. de 1834. Manuel d'actinologie ou de zoophytologie. Paris: .

4. Brook, G. 1889. Report on the Antipatharia. Reports on the scientific results of the voyage of H.M.S. Challenger during the years 1873-6, under the command of O.S. Nares and F.T. Thomson, Zool. 32: 5-222.

5. Bruguière, J.1. 1792. Encyclopédie méthodique, p.82.

6. Carter, H.J. 1880. On the Antipatharia (Milne-Edwards) with reference to Hydradendrium spinosum. Annls Mag. Nat. Hist. (5)6: 301-305.

7. Castorena Davis, V.M. 1979. Guide-lines for black coral exploitation. International Symposium For Fishery Educarion and Organization, Mexico. Vol 2: 1-9.

8. Church, R. and Buffington, E.C. 1969. Californian black coral. Oceans Mag. 1: 41-44.

9. Criales, M.M. 1980. Commensal caridean shrimps of Octocorallia and Antipatharia in Curacao and Bonaire with descriptions of a new species of Neopontonides. Uitgaven natuurw. Sudkring Surinome No. 103: 68-85.

10. Dana, J.D. 1846. Zoophytes. U.S. Exploring Expedition during the years 1838-42. Vol. 7.

10a. Dantan, J.L. 1921. Die Antipatharian. Archs. d'anat. comp. 17(2): 137-245.

10b. Deichmann, E. 1941. Coelenterates collected on the Pregidential Cruise of 1938. Smithsonian Misc. Call. 99(10): $1-17$.

11. Duchassaing, P. 1870. Revue des zoophytes et des spongiaires des Antilles. Paris:

12. Duchassaing, P. and Michelotti, J. 1860. Mémoire sur les coralliaires des Antilles. Memorie Accad. Sci. Torino (2) $19: 279-365$.

13. Duchassaing, P. and Michelotti, J. 1864. Supplément au mémoire sur les coralliaires des Antilles. Memorie Accad. Sci. Torino (2)23: $97-206$

14. Ellis, J, and Solander, D. 1786. The naturol histary of many curious and uncommon zoophytes, collected by the late John Ellis, systematically arranged and described by the late Daniel Solander. London: .

15. Esper, E.J.C. 1791-1797. Die Pllanzenthiere in Abbildungen nach der Natur mit Farben erleuchiet nebst Beschreibungen.

16. Faure, G. 1977. Distribution of coral communities on reef slopes in the Mascarene Archipelago, Indian Ocean. Mar. Res. Indones. 17: 73-97.

17. Forster Cooper, C. 1906. Antipatharia. Pp. 791-796 in J.S. Gardiner The fauna and geogrophy of the Maldives and Laccadive Archipelagoes, 2.
18. Forster Cooper, C. 1909. Repons of the Percy Sladen Trust Expedition to the Indian Ocean, 1905. Antipatharia. Trans. Linn. Soc. Lond. (2)12: 301-321.

19. Genin, A., Dayton, P.K., Lonsdale, P.K. and Spiess, F.N. 1986. Corals on seamount peaks provide evidence of current acceleration over deep-sea topography. Nature 322: 59-61.

20. Goldberg, W.M. 1976. Comparative study of the chemistry and structure of gorgonian and antipatharian coral skeletons. Mar. Biol. 35: 253-267.

21. Goldberg, W.M. and Taylor, G.T. 1989. Cellular structure and ultrastructure of the black coral Antipathes aperta. 1: Organisation of the tentacular epidermis and nervous system. J. Morphol. 202: 239-252.

22. Grange, K.R. 1988. Redescription of Antipathes opera, Totton, as ecological dominant in the southern fiords of New Zealand. N.Z. J. Zool. 15: 55-62.

23. Grange, K.R. 1990. Antipathes fiardensis, a new species of black coral from New Zealand. N.Z. J. Zool. 17: 279-282.

24. Grasshoff, M. 1981. Die Gorgonaria, Pennatularia und Antipatharia des Tiefwassers der Biskaya (Cnidaria, Anthozoa). Ergebnisse der französischen Expeditionen BioGas, PolyGas, Geomanche, Incal, Noratlante und Fahrten der "Thalassa". 1. Allgemeiner Teil. Bull. Mus. Natl. Hist. Nat. Sect. Zool. Biol. Ecal. Anim. 3: 731-766.

25. Grasshoff, M. 1981. Die Gorgonaria, Pennatularia und Antipatharia des Tiefwassers der Biskaya (Cnidaria, Anthozoa). Ergebnisse der französischen Expeditionen BioGas, PolyGas, Geomanche, Incal, Noratlante und Fahrten der "Thalassa". II. Taxonomische Teil. Bull. Mus. Natl. Hist. Nat. Sect. Zool. Biol. Ecol. Anim. 3: 941-978.

26. Grasshoff, M. 1985. Die Gorgonia und Antipatharia der Großen Meteor-Bank und der Josephine-Bank (Cnidaria: Anthozoa). Senckenb. Marit. 17: 65-87.

27. Grasshoff, M. 1988. The geographical and bathymetric distribution of the Gorgonacea and Antipatharia of St. Pauls and Amsterdam 1slands (Indian Ocean). Mesogee 48 115-124.

28. Grasshoff, M. 1989. Die Meerenge von Gibraltar als Faunen-Barriere: Die Gorgonaria, Pennatularia und Antipatharia der BALGIM-Expedition. Senckenb. Marit. 20: 201-223.

29. Gravier, C. 1918. Notes sur le Antipathaires du Golfe de Naples. Pubbl. Staz. zool. Napoli 2: 223-239.

29a. Gravier, C. 1921. Antipathaires provenant des campagnes des yachts Princesse-Alice et Hirondelle II 1903-1913.. Résultats des Campagnes scienrifiques accomplies sur son Yochl par Aben I, Prince of Monaco 39: 1-106.

30. Gray, J.E. 1857. Synopsis of the families and genera of axiferous zoophytes or barked corals. Proc. Zool. Soc. London 25: 278-294.

31. Gray, J.E. 1860. Notice of new corals from Madeira discovered by J.Y. Johnson, Esq. Ann. Mag. Not. Hist. (3) $6: 311$.

32. Gray, J.E. 1868. Descriptions of some new genera and species of Alcyonoid corals in the British Museum. Ann. Mag. Nat. Hist. (4)2: 441-445. 
33. Grigg, R.W. 1965. Ecological studies of black coral in Hawaii. Pac. Sci. 19: 244-260.

34. Grigg, R.W. 1984. Resource management of precious corals: a review and application to shallow water reef building corals. Mar. Ecol. 5: 57-74.

35. Grigg, R.W. and Opresko, D. 1977. Order Antipatharia. Black Corals. Bemice P. Bishop Mus. Spec. Publs. 64: 242-261

36. Guerriero, A., Dambrosio, M. and Pietra, F. 1988. Leiopathic acid, a novel optically active hydroxydocosapentaenoic acid, and related compounds from the black coral Leiopathes sp. of St Paul lsland (S. Indian Ocean). Helvetica Chimica Acta 71: 1094-1100.

36a. Hickson, S.J. 1905. Remarkable Coelenterata from the weat cosst of Ireland. Noture 73: 5 .

37. Hickson, S.J. 1907. The Alcyonaria, Antipatharia and Madreporis, collected by the "Huxley" from the north side of the Bay of Biscsy, in August 1906.J. Mar. Biol. Assoc. 8(1): 6-14.

38. Humes, A.G. 1969. Cyclopoid copepods associated with antipatharian coelenterates in Madagascar. Zool. Meded. 44: $1-30$.

39. Humes, A.G. 1979. Poecilostome copeopods associated with antipatharian coelenterates in the Moluccss. Beaufortio 28: $113-120$.

40. Johnson, J.Y. 1899. Notes on the Antipatharian corals of Madeira, with description of a new species and a new variety, and remarks on a specimen from the West-Indies in the British Museum. Proc. Zool. Soc. London 1899: 57.

41. Kenyon, J. 1984. Black coral of Cozumel. Sea Frontiers 30: $267-272$.

42. Kinoshita, K. 1910. On s new antipstharian Hexopothes heterosticha n.g. snd n.sp. Annotationes Zoologicae Japonenses 7: 231-234.

42a. Klunzinger, C.B. 1877. Die Koralthiere des Rothen Meeres.

43. Koch, G. von 1878. Mittheilungen über Coelenteraten: zur phylogenie der Antipatharis. Morph. Jb. 4 (Suppl.): 74-86.

44. Koch, G. von 1889. Die Antipathiden des Golfes von Neapel. Min. Zool. Sm. Neapel 9: 187-204.

45. Koch, W. 1886. Neue Anthozoan aus dem Golf von Guineo. Marburg (Elwert), 36 pp.

46. Krempf, A. 1905. Liste des Hexanthidea rapportes de l'ocean Indien (Golfe de Tadjourah) par M. Ch. Gravier. Bull. Mus. Nat. Hist. Paris 1905: 191-196.

47. Lacaze-Duthiers, H. 1865. Deuxième mémoire sur les Antipathaires (Antipathes vrais). Annls Sci. Nat. Zool. (5) $4: 5-61$.

48. Lamarck, J.B.P.A. de M. de 1816. Histoire naturelle des animaux sans vertebres, 2. Paris: Vendiere.

48a. Lamouroux, J.V.F. 1821. Exposition methodique des genres de l'ondre des polypiers, avec leur description el celle des principales especes, figurées dans 84 planches; les 63 premières appartenant d l'histoire naturelle des zoophytes d'Ellis et Solander. Paris.

49. Lamouroux, J.V.F., Bory de Saint-Vincent and Dealongchampa, J.A.E. 1824. Histoire naturelle dea zoophytea ou animaux rayonnéa....Encyclopedie méthodique, 2. Paris.
50. Lewis, J.8. 1978. Feeding mechanisms in black corals (Antipatharia).J. Zool. Lond. 186: 393-396.

51. Linnaeus, C. 1758. Systema naturae. Tenth edition.

52. Lütken, C. 1871. Antipathes arctica, en ny Sortkoral fra Polarhavet. Oversigi Kongl. Dansk. Vidensk. Selsk. Forhandl. 1871: 18-26.

53. Msrtinez, P. 1982. Preliminary report on blsck coral studies. Informe a estac. cient. Charles Darwin 1982: 209-220.

54. Martinez, P. snd Robinson, G. 1986. Studiez on the exploitation of black coral in the Galapagos Islands, Ecuador. Estac. Cient Charles Darwin Inf. Ann. 1983, 1986: 54-55.

55. Newton, E.C. and Bak, R.P.M. 1978. Ecologicsl sapects of Antipatharia in Curaçso. Association of Island Marine Laboratories of the Caribbean: 14th meeting. Santo Domingo, Dominican Republic Nov. 20-28, 1978.

56. Oakley, S.G. 1988. Settlement and growth of Anripathes pennacea on a shipwreck. Coral Reefs 7: 77-79.

57. Okamura, S.J. and Habe, T. 1976. The strange habitst of Rhikochilus teramachii. Jpn. J. Malacol. 35: 91-92.

58. Opresko, D.M. 1972. Redescription snd reevaluation of the antipatharians described by L.F. Pourtalè z. Bull. Mar. Sci. 22: $950-1017$.

59. Opresko, D.M. 1974. A study of the classification of the Antipatharia with redescription of 11 species. University Microfilms, Ann. Arbor. 1987: 1-194.

60. Opresko, D.M. 1976. Re-description of Antipathes panamensis (Coelenterata: Antipatharia). Poc. Sci. 30: 235240.

61. Opresko, D.M. and Genin, A. 1990. A new species of antipatharian (Cnidaria: Anthozoa) from Seamounts in the csstem north Pacific, Bull. Mar. Sci. 46: 301-310.

62. Pallss, P.S. 1766. Elenchua zoophytorum siatena generum adumbrationes generaliores et specierum cognitarum succinctas descriptiones cum selectis auctorum synonymia. Hagac-Comitum, XVI.

62a. Pasternak, F.A. 1958. [Deep sea Antipatharia of the Kurile-Kamchatka Depreasion.] Trudy Inst. Okeanol. 27: 180-191. (In Russian.)

62b. Pasternak, F.A. 1959. On the finding of Bathypathes potula Brook in high lstitudes in Antarctica. Inf. Bull. sowj. antarkt. Exped. 9: 366-367.

62c. Pasternak, F.A. 1961. [Pennatularia (Octo corallia) und Antipatharia (Hexacorallia), gesammelt auf der Sowjetischen Antarktis Exped. 1955-1958.] Trudy Inst. Okeanol. 46: 217-230. (In Russian.)

62d. Pasternsk, F.A. 1964. TThe deep-sea Pennatularians and Antipatharians obtained by R/S "Vitjaz" in the Indian Ocean and the resemblances between the fauna of the Indian Ocaen and the Pacific.] Trudy Inst. Okeanol. 69: 183-215. (In Rusaian.)

63. Pasternak, F.A. 1977. Antipatharia. Pp. 157-164 in Galatheo Repon 14: Scientific results of the Danish deep sea expedition round the world 1950-52. Scandinavian Science Press Ltd, Copenhagen. 
64. Pasternak, F.A. 1985. Specific composition and the ways of forming of the bottom fauna of isolated underwater rises. Gorgonarians \& antipatharians of the Seamounts Rockeway, Atlantis Plato, Great-Meteor \& Josephine (Atlantic Ocean). Trudy Inst. Okeanol. 120: 21-38.

64. Pax, F. 1915. Diagnosen neue Antipatharien. Zool. Anz 45: 598-601

65. Pax, F. 1916. Eine neue Annipathes-Art aus Westindien. Zool. Jahrb. Suppl. 11: 433-435.

65a. Pax, F. 1922. Die Antipatharien der Deutschen TiefseeExpedition. Wiss. Ergeb. deutsch Tiefsee-Exped. Valdivia 19(6): $6 \mathrm{pp}$.

66. Pax, F. 1931. Eine neue Srichopathes-Art des japanischen Litorals. Zool. Anz. 96: 321-325.1

67. Pax, F. 1932. Die Antipatharien und Madreporarien des arktischen Gebietes. Fauna Arcrica 6: 267-280.

68. Pax, F. 1934. Antipatharia. Tierwell der Nord und Ostsee III e 1: 22-38.

69. Pax, F. 1987. Ordre des antipathaires (Antipatharia Milne, Edwards el Haime, 1857; Antipathacea Dana, 1846 , Ceriantipatharia van Beneden, 1889). Pp. 189-256 in P.-P. Grassé (Ed.) Troité de zoologie. 3(3). Manson, Paris.

70. Not used.

71. Pesch, A.J. van 1910. Bijdragen tot de kennis van het genus Cirmipathes. Leiden. 96 pp.

72. Pesch, A.J. van 1914. The antipatharia of the Siboga Expedition. Siboga Expeditie 17(?): 1-258. Leiden, Netherlands: E.J. Brill.

73. Pourtales, L.F. de 1867. Contributions to the fauna of the Gulf Stream at great depths. Bull. Mus. Comp. Zool. 1(6): 103-120

74. Pourtales, L.F. de 1868. Contributions to the fauna of the Gulf Stream at great depths. Bull. Mus. Comp. Zool. 1(7): 121-142.

75. Pourtalès, L.F. de 1871. Deep-sea corals. III. Cat. Mus. Comp. Zool. 4: 1-93.

76. Pourtales, L.F. de 1874. Crinoids and corals. Zoological results of the "Hassler" Expedition. III. Cat. Mus. Comp. Zool. 8: 25-50.

77. Pourtalès, L.F. de 1878. Report on the corals of the "Blake" Expedition. Bull. Mus. Comp. Zool. 5: 197-212.

78. Pourtalèa, L.F. de $\mathbf{1 8 8 0}$. Reports on the resulta of dredging under the supervision of Alexander Agassiz in the Caribbean Sea, 1878-79, by the United States coast survey steamer "Blake". V1. Report on the corals and Antipatharia. Bull. Mus. Comp. Zool. 6(4): 95-120.

79. Robinson, G. 1982. Investigation of Galapagos antipatharian corals: preliminary results. Informe a estac. cient. Charles Darwin 1982: 192-208.

80. Roule, L. 1902 . Notice préliminaire sur lea Antipathaires provenant dea collections du Prince du Monaco. Mem. Soc. Zool. France 15: 228-239.

81. Not used.

82. Roule, L. 1905. Description dea Antipathairea et Cérianthairea recueillia par S.A.S. le Prince de Monaco dans l'Atlantique nord 1886-1902.. Résuleats des Campagnes Scientifiques accomplies sur son Yach par Alben I, Prince de Monaco. Fasc. XXX: 75-95.
83. Schmidt, H. 1973. On evolution in the Anthozoa. Proc 2nd Int. Symp. Coral Reefs, Brisbane, Australia. Vol 1. 533-560.

84. Schultze, L.S. 1896. Beiträg zur Systematik der Antipatharien. Abhandl. der Senckenberg. Naturf. Gesellsch. 23: 1-39.

85. Schultze, L.S. 1903. Die Antipatharien der Deutschen Tiefsee-Expedition 1898-1899. Wiss. Ergebn. deutsch. Tiefsee-Exped. Valdivia iii 3: 90-100. Jena, Fischer.

86. Schwartzman, G. and Opresko, D.M. 1992. Infrared spectrum of the skeletsl axis of antipatharian corals (Cnidaria: Anthozoa). Bull. Mar. Sci. 50: 352-356.

87. Silberfeld, E. 1909. Diagnosen neuer japanischer Antipatharien aus der Sammlung von Herm Prof. Doflein (München). Zool. Anz. 34: 760-763.

88. Silberfeld, E. 1909. Japanischer Antipatharien. Abh. Bayer Ak. Wiss. Math.-Physic. (Suppl.)7: 1-27.

89. Soest, R.W.M. van 1977. A catalogue of the coelenterate type specimens of the zoological museum of Amsterdam part 3: Antipatharia, Pennatulacea, Stolonifera, Telestacea, Alcyonacea . Beaufortia 26: 77-97.

90. Song I-1. 1987. A systematic study on the Korean Anthozoa. 10. Antipatharia (Hexacorallia). Korean J. Syst. Zool. 3: 63-73.

91. Summers, S.L.M. 1910. Antipatharians from the Indian Ocean. J. Roy. Microscop. Soc. ?: 273-281.

92. Thomson, J. 1905. Scotia Collections. Scottish Antarctic Expedition. Report on the Antipatharians. Proc. Roy. Physic. Soc. Edinburgh 16: 76-79.

92a. Thomson, J. 1907. Note on a large Antipatharian from the Faeroes. Proc. Roy. Physic. Soc. Edinburgh 17: 188-194.

93. Thomson, J. and Simpson, J.J. 1905. Report on the Antipatharia collected by Prof. Herdman at Ceylon, 1902. Report to the Govermment of Ceylon on the Pearl Oyster Fisheries of the Gulf of Manaar. Suppl. Rep. 4: 93-106.

94. Totton, A.K. 1923. Coelenterats of the British Antarctic "Terra Nova" Expedition. III. Antipatharia and their Cirriped commensals. Brit. Antarctic (Terra Nova) Exped., Nal. Hist. Rep., Zool. 5: 97-120.

95. Verrill, A.E. 1869. Notes on Radiata. Review of the corals and polyps of the west coast of America. Trans. Connecticul Acad. 1 : 377-558.

96. Verrill, A.E. 1928. Hawaiian shallow water Anthozoa Bull. Bishop Mus. Honoiulu 49: 1-30.

97. Warner, G.F. 1981. Species descriptions and ecological observations of black corals (Antipatharia) from Trinidad. Bull. Mar. Sci. 31: 147-163.

98. Wells, S.M., Pyle, R.M. and Collins, N.M. (Eds.) 1983. The IUCN invertebrate red daca book. IUCN, Gland, Switzerland. $632 \mathrm{pp}$.

98a. Whitelegge, T. and Hill, J.P. 1899. The Hydrozoa, Scyphozoa, Actinozoa and Vermes of Funafuti. Mem. Austral. Mus. (Misc. Publ.) 3: 371-394.

99. Woodley, J.D. and Emerson, R.H. 1987. Submersible and laboratory observations on Asteropora annulata from the ialand slope of north Jamaica. In: Burke, R.D. et. al. (Ed.). Echinadem biology; 6th Intemational Echinoderm Conference, Victoria, British Columbia, Canada, Auguat 23-28, 1987. A.A. Balkema: Rolterdam, Netherlands. 
100. Zhou Jin-ming and Zou Ren-lin 1988. IStudies on the antipathariana of China. 3. The genus Stichopathes.] Trop. Oceanol. 7: 63-70. (In Chinese.)

101. Zou Ren-lin and Zhou Jin-ming 1982. IStudies on the antipatharians of China. 1. The genus Cirrhipathes with the description of a new species.] Trop. Oceanol. 1: 92-91. (In Chinese.)
102. Zou Ren-lin and Zhou Jin-ming 1984. Antipatharians from Hong Kong waters with a description of a new species. Asian Marine Biology 1: 101-105. 


\section{Class: HYDROZOA}

\section{Order MILLEPORINA}

Family MILLEPORIDAE Fleming 1828

Millepora Linnaeus 1758

11

(Atlantic; Red Sea, East and South Africa [8,43]. Indian Ocean, north to southern India and Andaman and Nicobar 1slands [59]; south to Madagascar, Cocos-Keeling Islands [87] and south-western Australia [80]. South-east Asia. Pacific Ocean, north to southern Japan and Hawaiian Islands; south to the Great Barrier Reef of Australia and New Caledonia [75]; east to Tuamotu Archipelago [30])

American Samoa [50]; Anguilla; Antigua and Barbuda; Australia; Bahamas [47], Barbados; Belize [11]; Bermuda [47]; Brazil [49]; British Indian Ocean Territory [65,95]; British Virgin Islands [37]; Brunei; Cayman Islands; China [111, 112]; Christmas Island; Cocos (Keeling) Islands [77]; Colombia 162]; Comoros; Cook Islands; Costa Rica [33]; Cuba [48]; Djibouti; Dominicr; Dominican Republic; Ecuador; Egypt [64]; Ethiopia; Federated States of Micronesia; Fiji; French Polynesia [30,42]; Grenada; Guadeloupe; Guam; Haiti; Hawaiian Islands [100]; Honduras; Hong Kong [64a]; India [58,59]; Indonesia; Israel [64]; Jamaica [96]; Japan [78a]; Jordan [64]; Kenya [43]; Kiribati; Madagascar [56,57]; Malaysia [4]: including Sabah [101]; Maldives [20,65]; Marshall Islands; Martinique; Mauritius; Mexico [47]; Montserrat; Mozambique [8]; Myanmar; Nauru; Netherlands Antilles [47]; New Caledonia [102]; Nicaragua; Niue; Northern Marianas; Oman [66]; Palau; Panama [61]; Papua New Guinea; Philippines [79]; Puerto Rico; Reunion; Saint Kitts and Nevis; Saint Lucia; Saint Vincent and the Grenadines; Samoe; Saudi Arabia [1b]; Seychelles [65,99]; Singapore; Solomon Islands; Sri Lanka, Somalia; Sudan [64]; Tanzania [43]; Taiwan [34]; Thailand [36,72]; Tokelau; Tonga; Trinidad and Tobago; Turks and Caicos; Tuvalu; United Arab Emirates [9,67]; USA: Florida [100]; Vanuatu [76]; Virgin Islands of the United States; Venezuela; Wallis and Futuna; Yemen

A common and widespread coral, occurring in a wide range of reef habitats $[69,100]$.

Collected for use as a curio.

At least 48 nominal species. 9 species discussed in a recent ecological review [51]; possibly 13 species worldwide [6]

Millepora alcicornis Linnaeus 1758

Millepora boschmai De Weerdt \& Glynn 1991

Millepora braziliensis Verrill

Millepora complanata Lamarck 1816

Millepora cruzi Nemenzo 1975

Millepora dichotoma Forskàl 1775

Millepora exaesa Forskảl 1775

Millepora fasciculata?

Millepora intricata Milne Edwards 1857

Millepora latifolia Boschma 1948

Millepora murrayi?

Millepora nitida Verrill

Millepora platyphylla Hemprich \& Ehrenberg 1834

Millepora plicata?

Millepora pumila Dana 1846

Millepora ramosa?

Millepora squarrosa Lamarck 1816

Millepora striata Duchassaing \& Michelotti 1864

Millepora tenella Ortmann

Millepora tenera Boschma 1949 
Millepora tortuosa Dana 1846

Millepora tuberosa Boschma 1966

Millepora xishaensis Zou 1978

\section{Order STYLASTERINA}

Family STYLASTERIDAE Gray 1847

Adelopora Cairns 1982

I1

(Subantarctic; off Brazil; South Pacific seamounts, seamounts \& ridges between New Zealand and New Caledonia; Lord Howe seamount chain. 282-1,169m)

4 species $[13,27]$

Adelopora crassilabrum Cairns 1991

Adelopora fragilis Caims 1991

Adelopora moseleyi Cairns 1991

Adelopora pseudothyron Cairns 1982

Astya Stechow 1921

Philippines [26]; New Zealand [27]. 590-914m

2 species

Astya aspidopora Cairns 1991

Astya subviridis (Moseley 1879)

Calyptopora Boschma 1968

11

(New Zealand region. 216-2,010m [27])

At least 2 species

Calyptopora pachypoma (Hickson \& England 1905)

Calyptopora reticulata Boschma 1968

Calyptopora sinuosa Cairns 1991

Cheiloporidion Caims 1983

(Off Southeast South America. 642-1,137m [26])

1 species

Cheiloporidion pulvinatum Caims 1983

Congregopora Nielsen 1919

1 species

Congregopora nasiformis Nielsen 1919 
Conopora Moseley 1879

Il

(Indo-west Pacific; Subantarctic; Antarctic. 110-2,355m [26])

8 or 9 species

Conopora adeta Cairns 1987

Conopora anthohelia Cairns 1991 ?may not belong in this genus

Conopora candelabrum Caims 1991

Conopora dura Hickson \& England 1909

Conopora gigantea Cairns 1991

Conopora laevis (Studer 1878)

Conopora major Hickson \& England 1909

Conopora tetrastichopora Cairns 1991

Conopora unifacilais Caims 1991

Conopora verrucosa (Studer 1878)

Crypthelia Milne Edwards \& Haime 1849

(Cosmopolitan 140-2,789m [26, 27])

About 30 species

Crypthelia affinis Moseley 1879

Crypthelia balia Hickson \& England 1905

Crypthelia clausa Broch 1947

Crypthelia cryptotiema Zibrowius 1981

Crypthelia curvata Caims 1991

Crypthelia cymas Cairns 1986

Crypthelia dactylopoma Cairns 1986

Crypthelia eueides Cairns 1986

Crypthelia floridana Cairns 1986

Crypthelia formosa Caims 1983

Crypthelia fragilis Cairns 1983

Crypthelia gigantea Fisher 1938

Crypthelia glebulenta Cairns 1986

Crypthelia glossopoma Cairns 1986

Crypthelia insolita Caims 1986

Crypthelia japonica (Milne Edwards \& Haime 1849)

Crypthelia lacunosa Caims 1986

Crypthelia medioatlantica Zibrowius \& Cairns 1992

Crypthelia micropoma Caims 1985

Crypthelia papillosa Cairns 1986

Crypthelia peircei Pourtalès 1867

Crypthelia platypoma Hickson \& England 1905

Crypthelia polypoma Cairns 1991

Crypthelia pudica Milne Edwards \& Haime 1849

Crypthelia ramosa Hickson \& England 1905

Crypthelia robusta Cairns 1991

Crypthelia stenopoma Hickson \& England 1905

Crypthelia studeri Cairns 1991

Crypthelia tenuiseptata Cairns 1986

Crypthelia trophostega Fisher 1938

Crypthelia vascomarquesi Zibrowius \& Cairns 1992 
1 species

Cyclohelia lamellata Caims 1991

Distichopora Lamarck 1816

11

(Widely distributed: Western Atlantic; Red Sea, Indo-West Pacific, Hawaiian Islands; Galapagos 1slands [26,27]. $1-741 \mathrm{~m}$ depth)

It may be common beneath overhangs or in caves. Collected for use as a curio, and in the Philippines, for manufacture into beads for jewellery.

22 species

Distichopora (Haplomerismos) anceps Cairns 1978

Distichopora anomala Caims 1986

Distichopora barbadensis Pourtalès 1874

Distichopora borealis Fisher 1938

Distichopora cervina Pourtalès 1871

Distichopora coccinea Gray 1860

Distichopora contona Pourtalès 1878

Distichopora dispar Cairns 1991

Distichopora foliacea Pourtalès 1868

Distichopora gracilis Dana 1848

Distichopora irregularis Moseley 1881

Distichopora laevigranulosa Caims 1986

Distichopora livida Tenison-Woods 1879

Distichopora nitida Verrill 1864

Distichopora profunda Hickson \& England 1909

Distichopora providentiae (Hickson \& England 1909)

Distichopora rosalindae Cairns 1986

Distichopora serpens Broch 1942

Distichopora sulcata Pourtalès 1867

Distichopora uniserialis Cairns 1986

Distichopora violacea (Pallas 1766)

Distichopora yucatanensis Caims 1986

Errina Gray 1835

(North Atlantic, New Zealand region; Galapagos Islands; Antarctic. 6-1,772m [27])

23 species

Errina altispina Caims 1986

Errina antarctica (Gray 1872)

Errina aspera (Linnaeus 1767)

Errina atlantica Hickson 1912

Errina bicolor Caims 1991

Errina boschmai Caims 1983

Errina capensis Hickson 1912

Errina chathamensis Cairns 1991

Errina cheilopora Caims 1983

Errina cochleata Pourtalès 1867

Errina cruenta Boschma 1968

Errina dabneyi Pourtalès 1871

Errina dendyi Hickson 1912 
RL Ref

Errina fissurata Gray 1872

Errina gracilis Marenzeller 1903

Errina hicksoni Cairns 1991

Errina kerguelensis Broch 1942

Errina laevigata Caims 1991

Errina laterorifa Eguchi 1964

Errina macrogastra Marenzeller 1904

Errina novaeze(a)landiae Hickson 1912

Errina reticulata Cairns 1991

Errina rubra Broch 1942

Errina sinuosa Cairns 1991

Errinopora Fisher 1931

(Antarctic; Mauritius; Indian Ocean. 238-274m [26])

?1 species [26]

Errinopora cestoporina Cairns 1983

Errinopora cyclopora (Cairns 1983)

Errinopora latifundata Naumov 1960

Errinopora nanneca Fisher 1938

Errinopora porifera (Naumov 1960)

Errinopora pourtalesii (Dall 1884)

Errinopora stylifera (Broch 1935)

Errinopora zarhyncha Fisher 1938

Errinopsis Broch 1951

II

(Off southern South America. 250-77 Im [26])

2 species

Errinopsis fenestrata Caims 1983

Errinopsis reticulum Broch 1951

Gyropora Boschma 1960

11

South Africa 22m [26]

1 species

Gyropora africana Boschma 1960

Inferiolabiata Broch 1951

Subantarctic and Antarctic; New Zealand region. 87-2,100m [27]

3 species

Inferiolabiata labiata (Moseley 1879)

Inferiolabiata lowei Caims 1983

Inferiolabiata spinosa Cairns 1991 
15 species

Lepidopora acrolophos Cairns 1983

Lepidopora biserialis Cairns 1986

Lepidopora carinasa (Pourtalès 1867)

Lepidopora clavigera Cairns 1986

Lepidopora cryptocymas Cairns 1985

Lepidopora decipiens (Boschma 1964)

Lepidopora dendrostylus Caims 1991

Lepidopora diffusa (Boschma 1963)

Lepidopora glabra (Pourtalès I867)

Lepidopora granulosa (Cairns 1983)

Lepidopora hicksoni Boschma 1963

Lepidopora microstylus Cairns 1991

Lepidopora polystichopora Cairns 1985

Lepidopora sarmentosa (Boschma 1968)

Lepidopora symmetrica Caims 1991

Lepidotheca Cairns 1983

II

(Indo-West Pacific; Galapagos 1slands; Caribbean; New Zealand region; Subantarctic. 85-2,010m [26,27])

14 species

Lepidotheca altispina Cairns 1991

Lepidotheca brochi Caims 1986

Lepidotheca cervicornis (Broch 1942)

Lepidotheca chauliostylus Caims 1991

Lepidotheca fascicularis (Caims 1983)

Lepidotheca hachijoensis (Eguchi 1968)

Lepidotheca horrida (Hickson \& England 1905)

Lepidotheca inconsuta Caims 1991

Lepidotheca japonica (Eguchi 1968)

Lepidotheca macropora Cairns 1986

Lepidotheca pourtalesi Caims 1986

Lepidotheca ramosa (Hickson \& England 1905)

Lepidotheca robusta Cairns 1991

Lepidotheca tenuistylus (Broch 1942)

Paraerrina Broch 1942

(Mauritius; Indian Ocean. 238-274m [26])

1 species

Paraerrina decipiens Broch 1942

Phalangopora Kirkpatrick 1887

(Off Mauritius. 238-274m [26])

1 species

Phalangopora regularis Kirkpatrick 1887 
Pliobothrus Pourtalès 1868

II

(Northwest Atlantic; Indonesia. 80-1,600m [18,26])

At least 4 species

Pliobothrus echinatus Caims 1986

Pliobothrus gracilis Zibrowius \& Caims 1992

Pliobothrus spinosa (Hickson \& England 1905)

Pliobothrus symmetricus Pourtalès 1868

Pliobothrus tubulatus (Pourtalès 1867)

Pseudocrypthelia Caims 1983

II

Indonesia. 1,089m [15]

I species

Pseudocrypthelia pachypoma (Hickson \& England 1905)

Sporadopora Moseley 1879

II

New Zealand; Subantarctic. $122-1,498 \mathrm{~m}[26,27]$

3 species

Sporadopora dichotoma (Moseley 1876)

Sporadopora micropoma Caims 1991

Sporadopora mortenseni Broch 1942

Stellapora Cairns 1983

II

(Off Southeast South America. 205-1,647m [26])

1 species

Stellapora echinata (Moseley 1879)

Stenohelia Kent 1870

II

(West Pacific; Antipodes; Galapagos Islands; North Atlantic. 91-2,021m [18,26])

10 species

Stenohelia concinna Boschma 1964

Stenohelia conferia Boschma 1968

Stenohelia echinata Eguchi 1968

Stenohelia maderensis (Johnson 1862)

Stenohelia minima (Hickson \& England 1905)

Stenohelia pauciseptata Caims 1986

Stenohelia profunda Moseley 1881

Stenohelia robusta Boschma 1964

Stenohelia tiliata (Hickson \& England 1905)

Stenohelia umbonata (Hickson \& England 1905)

Stenohelia yabei (Eguchi 194I) 


\section{3 species}

Stylantheca papillosa (Dall 1884)

Stylantheca petrograpta (Fisher 1938)

Stylantheca porphyra Fisher 1931

\section{Stylaster Gray 1831}

(Worldwide, extending to the Arctic and Antarctic [30] 0.5-1,440m)

Shallow water species may be collected for use as curios, and in the Philippines, for manufacture into beads for jewellery

About 80 valid recent species [26]

Stylaster alaskanus Fisher 1938

Stylaster amphelioides Kent 1871

Stylaster antillarum Zibrowius \& Cairns 1982

Stylaster asper Kent 1871

Stylaster atlanticus Broch 1936

Stylaster aurantiacus Cairns 1986

Stylaster bellus (Dana 1848)

Stylaster bilobatus Hickson \& England 1905

Stylaster bithalamus Broch 1936

Stylaster blatteus (Boschma 1961)

Stylaster bocki Broch 1936

Stylaster boreopacificus Broch 1932

Stylaster boschmai (Eguchi 1965)

Stylaster brochi (Fisher 1938)

Stylaster brunneus Boschma 1970

Stylaster californicus (Verrill 1866)

Stylaster campylecus (Fisher 1938)

Stylaster cancellatus Fisher 1938

Stylaster carinatus Broch 1936

Stylaster complanatus Pourtalès 1867

Stylaster corallium Cairns 1986

Stylaster crassior Broch 1936

Stylaster densicaulis Moseley 1879

Stylaster dentatus Broch 1936

Stylaster divergens Marenzeller 1904

Stylaster duchassaingi Pourtalès 1867

Stylaster eguchii (Boschma 1966)

Stylaster elassotomus Fisher 1938

Stylaster erubescens Pourtalès 1868

Stylaster "eximius" Hickson \& England/?Fry 1905

Stylaster filogranus Pourtalès 1871

Stylaster flabelliformis (Lamarck 1816)

Stylaster galapagensis Caims 1986

Stylaster gemmascens (Esper 1794)

Stylaster gracilis Milne Edwards \& Haime 1850

Stylaster granulosus Milne Edwards \& Haime 1850

Stylaster hattorii (Eguchi 1968)

Stylaster horolgium Caims 1991

Stylaster ibericus Zibrowius \& Cairns 1992

Stylaster imbricatus Cairns 1991 
Stylaster incompletus (Tenison-Woods 1883)

Stylaster inornatus Cairns 1986

Stylaster laevigatus Cairns 1986

Stylaster lonchitis Broch 1947

Stylaster marenzelleri Cairns 1986

Stylaster maroccanus Zibrowius \& Cairns 1992

Stylaster marshae Cairns 1988

Stylaster microstriatus Broch 1936

Stylaster milleri Durham 1942

Stylaster miniatus (Pourtalès 1868)

Stylaster moseleyanus (Fisher 1938)

Stylaster multiplex Hickson \& England 1905

Stylaster nobilis (Kent 1871)

Stylaster norvegicus (Gunnerus 1768)

Stylaster papillosa (Dall 1884)

Stylaster papuensis Zibrowius 1981

Stylaster polymorphus Broch 1936

Stylaster polyorchis (Fisher 1938)

Stylaster profundiporus Broch 1936

Stylaster profundus (Moseley 1879)

Stylaster pulcher Quelch 1884

Stylaster punctatus Pourtalès 1871

Stylaster purpuratus (Naumov 1960)

Stylaster ramosus Broch 1936

Stylaster robustus (Caims 1983)

Stylaster rosaceus (Greef 1886)

Stylaster roseus (Pallas 1766)

Stylaster rossoamericanus Brandt 1872 ?nom. nud

Stylaster sanguineus ?Valenciennes in Milne Edwards \& Haime 1850

Stylaster scabiosus Broch 1935

Stylaster solidus Broch 1935

Stylaster spatula Cairns 1986

Stylaster stejnegeri (Fisher 1938)

Stylaster stell(ul)atus Steward 1878

Stylaster subviolaceus (Kent 1871)

Stylaster tenisonwoodsi Caims 1988

Stylaster venustus (Verrill 1870)

Stylaster verrillii (Dall 1884)

Systemopora Caims 1991

New Zealand [27]

Systemopora ornata Cairns 1991 


\section{Class: ANTHOZOA}

\section{Order HELIOPORACEA}

Family HELIOPORIDAE Moseley 1876

Heliopora Blainville 1830

II

(Red Sea, East and South Africa [8,43]. Indian Ocean, north to Maldives [95] and Indonesia; south to Madagascar and north-western Australia [75]. South-east Asia. Pacific Ocean, north to southern Japan and Marshall Islands; south to Great Barrier Reef and New Caledonia; east to Samoa [75])

Countries listed without reference numbers are within the distribution range shown in Veron [75]

American Samoa; Australia; British Indian Ocean Territory; Brunei; Christmas Island; Cocos (Keeling) Islands; Comoros; Egypt; Ethiopia; Federated States of Micronesia; Guam; Indonesia; Israel; Japan; Kenya; Madagascar; Malaysia: including Sabah [101]; Maldives; Marshall Islands; Mauritius; Mozambique; Nauru; New Caledonia; Niue; Northern Marianas; Palau; Papua New Guinea; Philippines; Réunion; Samoa; Saudi Arabia; Seychelles; Singapore; Solomon Islands; Somalia; Sudan; Tanzania; Taiwan; Thailand; Tokelau; Tuvalu; Vanuatu; Wallis and Futuna

Generally a fairly common coral that occurs in a wide range of reef habitats, especially in shallow areas [75, 100]. Collected for use as a curio and for decorative puposes. Also used as a building material I species

Heliopora coerulea (Pallas 1766)

\section{Order STOLONIFERA}

Family TUBIPORIDAE Ehrenberg 1828

Tubipora Linnaeus 1758

II

(Red Sea, East and South Africa [75]. Indian Ocean, north to Maldives and Indonesia; south to Madagascar and south-western Australia. South-east Asia. Pacific Ocean, north to southern Japan and Marshall Islands; south to Elizabeth Reef (eastern Australia); east to New Caledonia and Vanuatu [75, 100])

Countries listed without reference numbers are within the distribution range shown in Veron [75]

Australia; British Indian Ocean Territory; Brunei; Christmas Island; Cocos (Keeling) Islands; Comoros; Djibouti; Egypt; Ethiopia; Federated States of Micronesia; Guam; Indonesia; Israel; Japan; Kenya; Madagascar; Malaysia: including Sabah [101]; Maldives; Marshall Islands; Mauritius; Mozambique; Nauru; New Caledonia; Northern Marianas; Palau; Papua New Guinea; Philippines; Reunion; Saudi Arabia; Seychelles; Solomon Islands; Somalia; Sudan; Tanzania; Taiwan; Vanuatu; Yemen

A fairly common reef coral [75]. Collected for use as a curio and for other decorative purposes Four nominal species, probably only one true species [75] 


\section{Order SCLERACTINIA}

Family ASTROCOENIIDAE Koby 1890

Actinastrea d'Orbigny 1849

1 species

11

Actinastrea pectinata Pourtalés

Stephanocoenia Milne Edwards \& Haime 1848

Il

(Caribbean [61, 96] to Brazil [49]; Bermuda [68, 100])

Countries listed without reference numbers are within the distribution range shown in Smith [68] and Wood [100]

Anguilla; Antigua and Barbuda; Bahamas [47]; Barbados; Belize [11]; Bermuda [47]: Brazil [49]; British Virgin Islands [37]; Cayman Islands; Colombia [62]; Costa Rica [33]; Cuba [48]; Dominica; Dominican Republic; Grenada; Guadeloupe; Haiti; Honduras; Jamaica [96]; Martinique; Mexico [47]; Montserrat; Netherlands Antilles [47]; Nicaragua; Panama [61]; Puento Rico; Saint Kitts and Nevis; Saint Lucia; Saint Vincent and the Grenadines; Trinidad and Tobago; Turks and Caicos; USA: Florida [47]; Virgin 1slands of the United States; Venezuela

A small or medium-sized coral, occurring on most reefs [100]

1 species

Stephanocoenia michelinii Milne Edwards \& Haime 1848/ intersepta (Lamarck 1816)

Stylocoeniella Yabe and Sugiyama 1935

II

(Red Sea [64], East Africa [43]. Indian Ocean north to Maldives [95] and Mergui Archipelago, south to Madagascar [56], Cocos-Keeling Islands [77] and Houtman Abrolhos 1slands off western Australia [75]. South-east Asia. Pacific Ocean, north to southern Japan [40], south to Lord Howe Island, east to Northern Marianas, Marshall Islands and Tuamotu Archipelago $[42,75])$

Countries listed without reference numbers are within the distribution range shown in Veron [75]

American Samoa [50]; Australia; Bahrain [9,16]; British Indian Ocean Territory [65, 95]; Brunei; Christmas Island; Cocos (Keeling) Islands [77]; Comoros; Cook 1slands; Djibouti; Egypt [64]; Ethiopia; Federated States of Micronesia; Fiji; French Polynesia [30, 42]; Guam; Hong Kong [64a]; Indonesia; Japan [78a]; Kenya [43]; Kiribati; Madagascar [56, 57]; Malaysia [4]: including Sabah [101]; Maldives [20, 65]; Marshall Islands; Mauritius; Mozambique [8]; Myanmar; Nauru; New Caledonia [102]; Niue; Northern Marianas; Oman [66]; Palau; Papua New Guinea; Philippines [79]; Pitcairn Islands [54]; Reunion; Samoa; Saudi Arabia [1b]; Seychelles [65, 99]; Singapore; Solomon Islands; Somalia; Sudan [64]; Tanzania [43]; Taiwan [34]; Thailand [36, 72]; Tokelau; Tonga; Tuvalu; Vanuatu [76]; Wallis and Futuna

Stylocoeniella is unusual because it forms large colonies only on temperate reefs (e.g. southern Australia), near the limit of coral reef distribution. On tropical reefs it is uncommon and forms only small encrusting colonies [75]

3 species

Stylocoeniella armata (Ehrenberg 1834)

Stylocoeniella cocosensis Veron 1990

Stylocoeniella guentheri (Bassett-Smith 1890) 
Madracis Milne Edwards \& Haime 1849

II

(Widely distributed throughout most temperate and tropical seas: Western Atlantic, Caribbean $[10,61,96]$ to

Brazil [49], Azores, Cape Verde [70], Gulf of Guinea [49] Mediterranean [46]. Red Sea [64], Persian Gulf, Arabian Gulf [9]. Indian and Pacific Oceans to Hawaiian Islands [4], Galapagos Islands and Pacific coast of America [38])

Anguilla; Antigua and Barbuda; Australia; Azores [70]; Bahamas [47]; Bahrain [9,16]; Barbados; Belize [11]; Brazil [49]; British Indian Ocean Territory [65, 95]; British Virgin Islands [37]; Brunei; Cape Verde [70]; Cayman Islands; Chile; Costa Rica [33]; Cuba [48]; Dominica; Dominican Republic; Galapagos Islands [92, 93]; Grenada; Guadeloupe; Haiti; Hawaiian Islands [52]; Honduras; Indonesia; Israel [64]; Jamaica [96]; Madagascar [56, 57]; Malaysia: Sabah [101]; Martinique; Mexico [10]; Montserrat; Myanmar; Netherlands Antilles [47]; Nicaragua; Oman [66]; Panama [61]; Papua New Guinea; Philippines [79]; Pitcaim Islands [54]; Puerto Rico; Saint Kitts and Nevis; Saint Lucia; Saint Vincent and the Grenadines; Singapore; Thailand [36, 72]; Trinidad and Tobago; Turks and Caicos; USA(Florida; Virgin Islands of the United States; Venezuela There are probably 6 species in the Caribbean, where Madracis is a fairly common reef-building (hermatypic) coral [100]. In the Indo-Pacific there is probably only one reef-building species (M. kirbyi), occurring from Taiwan south to the Philippines [79], Thailand [36, 72], Borneo, Sulawesi, Moluccas, New Guinea and Australia: Great Barrier Reef [75], This species is rare and inconspicuous

Of the ahermatypic species, Madracis kauaiensis is endemic to the Hawaiian Islands [4]. There are in addition a number of non-reefal species of Madracis which occur in temperate waters [75, 46]

Madracis asanoi Yabe \& Sugiyama 1941

Madracis asperula Milne Edwards \& Haime 1850

Madracis brueggemanni (Ridley 1881)

Madracis decactis (Lyman 1859/7?)

Madracis formosa Wells 1973

Madracis interjecta Marenzeller 1906

Madracis kauaiensis Vaughan 1907

Madracis kirbyi Veron \& Pichon 1976

Madracis mirabilis (Duchassaing \& Michelotti 1860)

Madracis myriaster (Milne Edwards \& Haime 1849)

Madracis pharensis (Heller 1868)

Madracis profunda Zibrowius 1980

Madracis senaria Wells 1974

Palauastrea Yabe \& Sugiyama 1941

11

(Houtman Abrolhos Islands off western Australia [80], Malaysia [101], Philippines [79], north to Ryukyu Archipelago, east to Palau Islands; south to north-facing coasts of Java, Sumatra, Irian Jaya and Papua New Guinea. Great Barrier Reef [75])

Countries listed without reference numbers are within the distribution range shown in Veron [75]

Australia; Indonesia; Japan [78a]; Malaysia: Sabah [101]; Palau; Papua New Guinea; Philippines [79]; Singapore; Vanuatu [76]

Restricted to sheltered reef areas on a sandy substratum where it may be common; otherwise not abundant. This coral is often confused underwater with Porites and may be more widely distributed [75, 100]

1 species

Palauastrea ramosa Yabe \& Sugiyama 1941 
(Red Sea [64], Persian Gulf, East and South Africa [1b, 12]. Indian Ocean, north to Arabian Gulf [9] Lacadives, Andaman and Nicobar Islands [59] and Mergui Archipelago, south to Madagascar [56], Cocos-Keeling Islands [77] and South-Western tip of Australia [75]. South-east Asia. Pacific Ocean, north to Japan [40], Midway Islands, Hawaiian Islands [52] and California [69], south to Lord Howe Island and Kermadec Islands, east to Pitcairn Islands Island [75], Easter lsland [90], Galapagos Islands and Colombia [38])

Countries listed without reference numbers are within the distribution range shown in Veron [75]

American Samoa [50]; Australia; Bahrain [9,16]; British Indian Ocean Territory [65, 95]; Brunei; Chile: Easter Island [90]; China [111, 112]; Christmas Island; Cocos (Keeling) 1slands [77]; Colombia [62]; Comoros; Cook Islands; Costa Rica [33]; Djibouti; Ecuador: Galapagos Islands; Egypt [64]; Ethiopia; Federated States of Micronesia; Fiji; French Polynesia [30, 42]; Guam; Hawaiian Islands [52]; Hong Kong [64a]; India [58, 59]; Indonesia; Iran; Israel [64]; Jordan [64]; Japan [78a]; Kenya [43]; Kiribati; Madagascar [56, 57]; Malaysia [4]: including Sabah [101]; Maldives [20, 65]; Marshall Islands; Mauritius; Mozambique [8]; Myanmar; Nauru; New Caledonia [102]; New Zealand: Kermadec Islands; Niue; Northern Marianas; Oman [66]; Palau; Papua New Guinea; Philippines [79]; Pitcairn Jslands [54]; Qatar; Reunion; Samoa; Saudi Arabia [1b]; Seychelles [65, 99]; Singapore; Solomon Islands; Sri Lanka; Somalia; Sudan [64]; Tanzania [43]; Taiwan [34]; Thailand [36, 72]; Tokelau; Tonga; Tuvalu; United Arab Emirates [9, 67]; USA: Gulf of California [52]; Vanuatu [76]; Wallis and Futuna; Yemen

A hardy, reef-building coral. $P$. damicornis, $P$. verrucosa and $P$. eydouxi are the commonest and most widely distributed species. Collected for use as a curio.

Approximately 35 nominal species; an estimated 7-10 valid species [75], of which 6 are established

Pocillopora ankeli Scheer \& Pillai 1975

Pocillopora aspera Verrill 1869

Pocillopora capitata Verrill 1864

Pocillopora clavaria Ehrenberg

Pocillopora damicornis (Linnaeus 1758)

Pocillopora diomedeae Vaughan 1906

Pocillopora eydouxi Milne Edwards \& Haime 1860

Pocillopora frondosa Verrill 1869

Pocillopora informis Dana 1846

Pocillopora ligulata Dana

Pocillopora mauritiana Brüggemann

Pocillopora meandrina Dana 1846

Pocillopora molokensis Vaughan 1907

Pocillopora plicata Dana 1846

Pocillopora porosa Verrill 1869

Pocillopora setchelli Hoffmeister 1929

Pocillopora solida Quelch 1886

Pocillopora squarrosa Dana 1846

Pocillopora verrucosa (Ellis and Solander 1786)

Pocillopora woodjonesi Vaughan 1918

Seriatopora Lamarck 1816

(Red Sea [64], East and South Africa [8, 43], Indian Ocean north to Maldives [95], Andaman and Nicobar Islands [59] and Mergui Archipelago; south to Madagascar [56], Cocos-Keeling Islands [77], Ningaloo Reefs (western Australia) [80]. South-East Asia. Pacific Ocean, north to Ryukyu Islands, south to Lord Howe Island, east to Phoenix Islands and Samoa [75])

Countries listed without reference numbers are within the distribution range shown in Veron [75]

American Samoa [50]; Australia; British Indian Ocean Territory [65, 95]; Brunei; Myanmar; Christmas Island; Cocos (Keeling) Islands [77]; Comoros; Egypt [64]; Ethiopia; Federated States of Micronesia; Fiji; Guam; Hong 
Kong [64a]; India [58, 59]; Indonesia; Israel [64]; Jordan; Japan [78a]; Kenya [43]; Kiribati; Madagascar [56, 57]; Malaysia [4]: including Sabah [101]; Maldives [20, 65]; Marshall Islands; Mauritius; Mozambique [8]; Nauru; New Caledonia [102]; Northern Marianas; Oman [66]; Palau; Papua New Guinea; Philippines [79]; Pitcaim Islands [54]; Reunion; Samoa; Saudi Arabia [1b]; Seychelles [65, 99]; Singapore; Solomon Islands; Somalia; Sudan [64]; Tanzania [43]; Taiwan [34]; Thailand [36, 72]; Tokelau; Tuvalu; Vanuatu [76]; Wallis and Futuna; Yemen

Collected for use as a curio.

Twenty-six nominal species, possibly only 2 very variable species [87]

Seriatopora caliendrum Ehrenberg 1834

Seriatopora crassa Quelch

Seriatopora hystrix Dana 1846

Seriatopora spinosa Milne Edwards \& Haime

Seriatopora stellata Quelch

Seriatopora stricta Brüggemann

Seriatopora subulata Lamarck 1816

\section{Stylophora Schweigger 1819}

(Red Sea [64], Persian Gulf, East and South Africa [8, 43]. Indian Ocean, north to Arabian Gulf [9], Lacadives, Andaman and Nicobar Islands [59] and Mergui Archipelago; south to Madagascar [56], and Shark Bay (western Australia) [80]. South-East Asia. Pacific Ocean, north to Japan [78a], south to Lord Howe Island, east to Line Islands [75], Tuamotu Archipelago [42] and Pitcaim Islands Island)

Countries listed without reference numbers are within the distribution range shown in Veron [75]

American Samoa [50]; Australia; Bahrain [9,16]; British Indian Ocean Territory [65, 95]; Brunei; Myanmar;Christmas Island; Comoros; Cook 1slands; Djibouti; Egypt [64]; Ethiopia; Federated States of Micronesia; Fiji; French Polynesia [30, 42]; Guam; Hong Kong [64a]; India [58, 59]; Indonesia; Iran; Israel [64]; Jordan; Japan [78a]; Kenya [43]; Kiribati; Madagascar [56, 57]; Malaysia [4]: including Sabah [101]; Maldives [20, 65]; Marshall Islands; Mauritius; Mozambique [8]; Nauru; New Caledonia [102]; Niue; Northern Marianas; Oman [66]; Palau; Papua New Guinea; Pitcaim Islands; Philippines [79]; Qatar; Reunion; Samoa; Saudi Arabia [1b]; Seychelles [65, 99]; Singapore; Solomon Islands; Sri Lanka; Somalia; Sudan [64]; Tanzania [43]; Taiwan [34]; Thailand [36, 72]; Tokelau; Tonga; Tuvalu; United Arab Emirates [9, 67]; Vanuatu [76]; Wallis and Futuna; Yemen

Collected for use as a curio.

Twenty-four nominal species; possibly 4 valid species [75], of which 3 are established

Stylophora compressa Gardiner 1898

Stylophora contorta Ley

Stylophora kuehlmanni Scheer \& Pillai

Stylophora lobata Gardiner 1898

Stylophora mamillata Scheer \& Pillai 1983

Stylophora pistillata (Esper 1795)

Stylophora rugosa Gardiner 1898

Stylophora stellata Verrill 1864

Stylophora wellsi Scheer 1964 


\section{Acropora Oken 1815}

Il

(Caribbean [61, 96]. Red Sea [64], Persian Gulf, East and South Africa [8, 43]. Indian Ocean north to Arabian Gulf [9], Gulf of Kutch (north-west India), Andaman and Nicobar 1slands [59] and Mergui Archipelago; south to Madagascar [56], Cocos-Keeling 1slands [77] and south-western tip of Australia [80]. South-east Asia. Pacific Ocean; north to southern Japan [40], Midway Islands and Hawaiian Islands [52]; south to Lord Howe Island, Kermadec Islands and Pitcairn Islands Island [75])

Countries listed without reference numbers are within the distribution range shown in Veron [75]

American Samoa [50]; Anguilla; Antigua and Barbuda; Australia; Bahamas [47]; Bahrain [9, 16]; Barbados; Belize [11]; Brazil [49]; British Indian Ocean Territory [65, 95]; British Virgin Islands [37]; Brunei; Cayman Islands; China [111, 112]; Christmas Island; Cocos (Keeling) Islands [77]; Colombia [62]; Comoros; Cook 1slands; Costa Rica [33]; Cuba [48]; Djibouti; Dominica; Dominican Republic; Egypt [64]; Ethiopia; Federated States of Micronesia; Fiji; French Polynesia [30, 42]; Grenada; Guadeloupe; Guam; Haiti; Hawaiian Islands [52]; Honduras; Hong Kong [64a]; India [58, 59]; Indonesia; Iran; Israel [64]; Jamaica [96]; Japan [78a]; Jordan [64]; Kenya [43]; Kiribati; Kuwait; Madagascar [56, 57]; Malaysia [4]: including Sabah [101]; Maldives [20, 65]; Marshall Islands; Martinique; Mauritius; Mexico [47]; Montserrat; Mozambique [8]; Myanmar; Nauru; Netherlands Antilles [47]; New Caledonia [102]; New Zealand; Nicaragua; Niue; Northern Marianas; Oman [66]; Palau; Panama [61]; Papua New Guinea; Philippines [79]; Pitcaim Islands [54]; Puerto Rico; Qatar; Reunion; Saint Kitts and Nevis; Saint Lucia; Saint Vincent and the Grenadines; Samoa; Saudi Arabia [1b]; Seychelles [65, 99]; Singapore; Solomon Islands; Sri Lanka; Somalia; Sudan [64]; Tanzania [43]; Taiwan [34]; Thailand [36, 72]; Tokelau; Tonga; Trinidad and Tobago; Turks and Caicos; Tuvalu; United Arab Emirates [9, 67]; USA: California, Florida; Vanuatu [76]; Virgin Islands of the United States; Venezuela; Wallis and Futuna; Yemen

Acropora is one of the commonest and most widespread reef-building genera. In India and South-East Asia in particular, Acropora is collected and fired in kilns to produce quicklime, a constituent of cement. Also widely collected for use as a curio. There are 368 nominal species but variability withir species has led to considerable taxonomic confusion. The true number is unknown, but likely to be in the region of one hundred [75]. Only 3 species occur in the Western Atlantic [100], the rest in the lndo-Pacific. 76 species have been recognised from Australia [75], of which about one sixth have not been recorded elsewhere in the Indo-Pacific

*nominal species occurring in the Philippines, but taxonomic status not fully investigated [79]

Acropora abrolhosensis Veron 1985

Acropora acervata (Dana 1846)

Acropora aculeus (Dana 1846)

Acropora acuminata (Verrill 1864)

Acropora akajimensis Veron 1990

Acropora alces (Dana 1846)

Acropora angulata (Quelch 1886)*

Acropora anthocercis (Brook 1893)

Acropora appressa (Ehrenberg 1834)

Acropora arbuscula (Dana 1846)

Acropora aspera (Dana 1846)

Acropora attenuata (Brook)

Acropora austera (Dana 1846)

Acropora azurea Veron \& Wallace 1984

Acropora brueggemanni (Brook 1893/Studer 1878?)

Acropora bushyensis Veron \& Wallace 1984

Acropora canalis (Quelch)

Acropora capillaris (Klunzinger)

Acropora cardenae Wells 1985

Acropora carduus (Dana 1846)

Acropora caroliniana Nemenzo 1976 
Acropora cerealis (Dana 1846)

Acropora cervicornis (Lamarck 1816)

Acropora ceylonica (Ortmann)

Acropora chesterfieldensis Veron \& Wallace 1984

Acropora clathrata (Brook 1893)

Acropora conigera (Dana)

Acropora convexa (Dana 1846)

Acropora copiosa Nemenzo 1967

Acropora corymbosa (Lamarck 1816)

Acropora crassa (Milne Edwards \& Haime)

Acropora crateriformis (Gardiner 1899)

Acropora cuneata (Dana 1846)

Acropora cuspidata (Dana 1846)

Acropora cyclopea (Dana 1846)

Acropora cytherea (Dana 1846)

Acropora danai (Milne Edwards \& Haime 1860)

Acropora demani (Rebberg 1892)*

Acropora dendrum (Bassett-Smith 1890)

Acropora diffusa (Verrill 1864)

Acropora digitifera (Dana 1846)

Acropora diomedeae Vaughan 1906

Acropora divaricata (Dana 1846)

Acropora donei Veron \& Wallace 1984

Acropora dumosa (Brook)

Acropora echinata (Dana 1846)

Acropora effluens (Dana)

Acropora elegans Milne Edwards \& Haime 1860

Acropora elliptica (Rehberg 1892)*

Acropora elseyi (Brook 1892)

Acropora eurystoma (Klunzinger 1879)

Acropora exquisita Nemenzo 1971

Acropora flabelliformis Milne Edwards \& Haime

Acropora florida (Dana 1846)

Acropora formosa (Dana 1846)

Acropora forskalii (Ehrenberg 1834)

Acropora gemmifera (Brook 1892)

Acropora glauca (Brook 1893)

Acropora glochiclados (Brook 1893)

Acropora gonagra Milne Edwards \& Haime

Acropora gracilis (Dana 1846)

Acropora grandis (Brook 1892)

Acropora granulosa (Milne Edwards \& Haime 1860)

Acropora hemprichii Ehrenberg 1834

Acropora heteroclados (Brook 1893)

Acropora horrida (Dana 1846)

Acropora humilis (Dana 1846)

Acropora hyacinthus (Dana 1846)

Acropora implicata (Dana 1846)

Acropora indica (Brook)

Acropora insignis Nemenzo 1967

Acropora kirstyaeli? Veron \& Wallace 1984

Acropora latistella (Brook 1893)

Acropora lianae Nemenzo

Acropora listeri (Brook 1893)

Acropora longicyathus (Milne Edwards \& Haime 1860)

Acropora loripes (Brook 1892) 
Acropora lovelli Veron \& Wallace 1984 Acropora lutkeni Crossland 1952 Acropora luzonica (Verrill 1902)* Acropora magnifica Nemenzo Acropora microclados (Ehrenberg 1834) Acropora microphthalma (Verrill 1869) Acropora millepora (Ehrenberg 1834) Acropora mirabilis (Quelch 1886) Acropora monticulosa (Brüggemann 1879) Acropora multiacuta Nemenzo 1967 Acropora nana (Studer 1878) Acropora nasuta (Dana 1846) Acropora nobilis (Dana 1846) Acropora ocellata (Klunzinger 1879) Acropora oligocyathus (Brook) Acropora pagoensis Hoffmeister 1925 Acropora palifera (Lamarck 1816) Acropora palmata (Lamarck 1816) Acropora palmerae Wells 1954 Acropora paniculata Verrill 1902 Acropora parilis Quelch 1816 Acropora parvistella (Verrill 1864) Acropora pharaonis (Milne Edwards \& Haime 1860) Acropora philippinensis (Rehberg 1892)* Acropora platycyathus (Brook 1893) Acropora polymorpha (Brook) Acropora polystomo (Brook 1891) Acropora profusa Nemenzo Acropora prolifera (Lamarck 1816) Acropora prostrata (Dana 1846) Acropora pruinosa (Brook 1893) Acropora pulchra (Brook 1893) Acropora pumila (Verrill 1866) Acropora rambleri (Bassett-Smith 1890) Acropora ramiculosa (Dana 1846) Acropora retusa (Dana 1846) Acropora robusta (Dana 1846) Acropora rosaria (Dana 1846) Acropora rotumana (Gardiner 1899) Acropora samoensis (Brook 1891) Acropora sanguinolenta? Acropora sarmentosa (Brook 1892) Acropora scandens (Klunzinger 1879) Acropora schmitti Wells 1950 Acropora secale (Studer 1878) Acropora sekiseiensis Veron 1990 Acropora selago (Studer 1878) Acropora solitaryensis Veron \& Wallace 1984 Acropora spicifera (Dana 1846) Acropora splendida Nemenzo 1967 Acropora squamosa (Brook) Acropora squarrosa (Ehrenberg 1834) Acropora stigmataria Milne Edwards \& Haime Acropora stoddarti Pillai \& Scheer 1976 Acropora striata (Verrill 1866) Acropora studeri Brook 
Acropora subglabra (Brook 1891)

Acropora subulata (Dana 1846)

Acropora syringodes (Brook 1893)

Acropora tanegashimensis Veron 1990

Acropora tenella (Brook 1892)

Acropora tenuis (Dana 1846)

Acropora teres (Verrill 1866)

Acropora thurstoni (Brook)

Acropora tortuosa (Dana 1846)

Acropora tuberculosa Milne Edwards \& Haime

Acropora tubigera (Horn 1860)

Acropora tubicinaria (Dana 1846)

Acropora tubulosa (Ehrenberg)

Acropora tumida Verrill 1866

Acropora turbinata (Dana)

Acropora turgida (Verrill 1866)

Acropora valenciennesi (Milne Edwards \& Haime 1860)

Acropora valida (Dana 1846)

Acropora vaughani Wells 1954

Acropora verweyi Veron \& Wallace 1984

Acropora wallaceae Veron 1990

Acropora willisae Veron \& Wallace 1984

Acropora yongei Veron \& Wallace 1984

Anacropora Ridley 1884

11

(Aldabra and the Seychelles in the Western Indian Ocean; north to the Maldives, southern India and Mergui Archipelago [75]; south to Cocos-Keeling Islands [77] and north-western Australia [80]. South-east Asia. Pacific Ocean north to Ryukyu 1slands, south to the Great Barrier Reef and Vanuatu [76]; east to the Marshall 1slands and Fiji [75])

Countries listed without reference numbers are within the distribution range shown in Veron [75]

Australia; British Indian Ocean Territory [65, 95]; Brunei; China [111, 112]; Christmas 1sland; Cocos (Keeling) Islands [77]; Federated States of Micronesia; Hong Kong [64a]; Indonesia; Japan [78a]; Malaysia: Sabah [101]; Maldives [20, 65]; Myanmar; Palau; Papua New Guinea; Philippines [79]; Seychelles [65, 99]; Singapore; Solomon Islands; Taiwan [34]; Thailand [36, 72]; Vanuatu [76]

Anacropora appears to be restricted to soft substrata on coral reefs, and is uncommon or rare. A. reticulata, known only from the central Great Barrier Reef, is reported to be very rare [75].

Ten nominal species, possibly 6 valid species (5 listed below), of which 4 occur in Australia [75]

Anacropora forbesi Ridley 1884

Anacropora gracilis Quelch

Anacropora matthai Pillai 1973

Anacropora puenogalerae Nemenzo 1964

Anacropora reptans?

Anacropora reticulata Veron \& Wallace 1984

Anacropora spinosa Rehberg 1892 
Astreopora Blainville 1830

II

(Red Sea [64], Persian Gulf, East and South Africa [8, 43]. Indian Ocean north to Lacadives, Gulf of Mannar (southern India) [59] and Mergui Archipelago; south to Madagascar [56], Cocos-Keeling Islands [77] and Houtman Abrolhos Islands [80]. South-east Asia. Pacific Ocean, north to southern Japan [40]; south to Lord Howe Island; east to Tuamotu Archipelago [42] and Pitcairn 1slands Island [75])

Countries listed without reference numbers are within the distribution range shown in Veron [75]

American Samoa [50]; Australia; Bahrain [9,16]; British Indian Ocean Territory [65, 95]; Brunei; China [111, 112]; Christmas Island; Cocos (Keeling) Islands [77]; Comoros; Cook Islands; Djibouti; Egypt [64]; Ethiopia; Federated States of Micronesia; Fiji; French Polynesia [30, 42]; Guam; Hong Kong [64a]; India [58, 59]; Indonesia; Iran; Israel [64]; Jordan; Japan [78a]; Kenya [43]; Kuwait; Madagascar [56, 57]; Malaysja [4]: including Sabah [101]; Maldives [20, 65]; Marshall Islands; Mauritius; Mozambique [8]; Myanmar; Nauru; New Caledonia [102]; Niue; Northern Marianas; Oman [66]; Palau: Papua New Guinea; Philippines [79]; Pitcairn Islands [54]; Qatar; Reunion; Samoa; Saudi Arabia [1b]; Seychelles [65, 99]; Singapore; Solomon Islands; Somalia; Sudan [64]; Tanzania [43]; Taiwan [34]; Thailand [36, 72]; Tokelau; Tonga; Tuvalu; United Arab Emirates [9,67]; Vanuatu [76]; Wallis and Futuna; Yemen

A. myriophthalma is the commonest and most widespread species, other species are mostly uncommon or rare [75]. Collected for use as a building material.

28 nominal species, approximately 15 valid species, of which 9 occur in Australia [75]. 11 established species are listed below

Astreopora cucullata Lamberts 1980

Astreopora expansa Brüggemann 1877

Astreopora explanata Veron 1985

Astreopora gracilis Bernard 1896

Astreopora incrustans Bernard 1896

Astreopora lambertsi Moll \& Best 1984

Astreopora listeri Bernard 1896

Astreopora macrostoma Veron \& Wallace 1984

Astreopora moretonensis Veron \& Wallace 1984

Astreopora myriophthalma (Lamarck 1816)

Astreopora ocellata Bernard 1896

Astreopora ovalis Bernard

Astreopora punctifera Bernard

Astreopora scabra Lamberts 1982

Astreopora suggesta Wells 1954

Astreopora tabulata Gardiner 1898

Montipora Blainville 1830

II

(Red Sea [64], Persian Gulf, East and South Africa [8, 43]. Indian Ocean north to Arabian Gulf [9], Lacadives [59] and Mergui Archipelago; south to Madagascar [56], Cocos-Keeling Islands [77] and Houtman Abrolhos Islands (western Australia] [80]. South-east Asia, north to Japan [40], Midway Islands, Hawaiian Islands [52]; south to Lord Howe Island, Kermadec Islands; east to Marquesas [42] and Pitcaim 1slands Island [75])

Countries listed without reference numbers are within the distribution range shown in Veron [75]

American Samoa [50]; Australia; Bahrain [9,16]; British Indian Ocean Territory [65, 95]; Brunei; China [111. 112]; Christmas Island; Cocos (Keeling) Islands [77]; Comoros; Cook Islands; Djibouti; Egypt [64]; Ethiopia; Federated States of Micronesia; Fiji; French Polynesia [30, 42]; Guam; Hawaiian Islands [52]; Hong Kong [64a]; India [58, 59]; Indonesia; Iran; Israel [64]; Jordan [64]; Japan [78a]; Kenya [43]; Kiribati; Madagascar [56, 57]; Malaysia [4]: including Sabah [101]; Maldives [20, 65]; Marshall Islands; Mauritius; Mozambique [8]; Myanmar; Nauru; New Caledonia [102]; New Zealand: Kermedec Islands; Niue; Northern Marianas; Oman [66]; Palau; Papua New Guinea; Philippines [79]; Pitcaim Islands [54]; Qatar; Reunion; Samoa; Saudi Arabia [1b]; Seychelles [65, 99]; Singapore; Solomon Islands; Sri Lanka; Somalia; Sudan [64]; Tanzania [43]; Taiwan 
[34]; Thailand [36, 72]; Tokelau; Tonga; Tuvalu; United Arab Emirates [9, 67]; Vanuatu [76]; Wallis and Futuna; Yemen

Montipora is an important and widespread reef coral. Collected for use as curios.

There are 211 nominal species, but variability within species has led to considerable taxonomic confusion. The number of valid species is unknown, but the $\$ 7$ listed below are reasonably well established. At least $\mathbf{3 8}$ have been recognised from Australia, of which about one sixth bave not been recorded from elsewhere in the Indo-Pacific [75]

Montipora acutata Bernard 1897

Montipora aequituberculata Bernard 1897

Montipora alsasepta Nemenzo 1967

Montipora alveopora Bernard

Montipora angulata (Lamarck 1816)

Montipora aspera Verrill 1872

Montipora australiensis Bernard 1897

Montipora bernardi Vaughan 1907

Montipora berryi Hoffmeister 1925

Montipora bilaminata Bernard 1897

Montipora cactus Bernard 1897

Montipora calcarea Bernard 1897

Montipora caliculasa (Dana 1846)

Montipora capitata (Dana 1846)

Montipora capricornis Veron 1985

Montipora cebuensis Nemenzo 1976

Montipora circumvallata (Ehrenberg 1834)

Montipora cocosensis Vaugban

Montipora columnaris Bernard 1898

Montipora complanata (Lamarck 1816)

Montipora confusa Nemenzo 1967

Montipora corbettensis Veron \& Wallace 1984

Montipora crassituberculata Bernard 1897

Montipora cristagalli (Ehrenberg)

Montipora culiculata Bernard

Montipora danae (Milne Edwards \& Haime 1851)

Montipora digitata (Dana 1846)

Montipora dilitata Studer 1901

Montipora edwardsi (Bernard 1897)

Montipora efflorescens Bernard 1897

Montipora effusa Dana 1846

Montipora ehrenbergi Verrill 1872

Montipora elschneri Vaughan 1918

Montipora explanata Brüggemann

Montipora explanulata Bernard

Montipora exserta Quelch

Montipora flabellasa Studer 1901

Montipora florida Nemenzo 1967

Montipora floweri Wells 1956

Montipora foliosa (Pallas 1766)

Montipora foveolata (Dana 1846)

Montipora friabilis Bernard 1897

Montipora fruticosa Bernard

Montipora gaimardi Bernard 1897

Montipora gracilis Klunzinger 1879

Montipora grandifolia Bernard 1892

Montipora granifera Bernard 
Montipora granulosa Bernard 1897

Montipora grisea Bernard 1897

Montipora hirsuta Nemenzo 1967

Montipora hispida (Dana 1846)

Montipora hoffmeisteri Wells 1954

Montipora incognita Bernard

Montipora incrassata (Dana 1846)

Montipora informis Bernard 1897

Montipora jonesi Pillai

Montipora levis Quelch

Montipora lichen (Dana 1846)

Montipora lobulata Bernard 1897

Montipora mactanensis Nemenzo 1979

Montipora malampaya Nemenzo 1967

Montipora maldivensis Pillai \& Scheer

Montipora manauliensis Pillai

Montipora marshallensis Wells 1954

Montipora millepora Crossland 1952

Montipora mollis Bernard 1897

Montipora monasteriata (Forskảl 1775)

Montipora multipapillosa Nemenzo 1983

Montipora nodosa (Dana 1846)

Montipora orientalis Nemenzo 1967

Montipora palmata (Dana 1846)

Montipora papillosa (Lamarck)

Montipora parula Verrill 1869

Montipora paupera Mamen

Montipora peltiformis Bernard 1897

Montipora perforata Bernard

Montipora poritiformis Verrill 1866

Montipora profunda Bernard

Montipora rigida Verrill 1866

Montipora samarensis Nemenzo 1967

Montipora saxea Bernard

Montipora scabricula (Dana 1846)

Montipora setosa Nemenzo 1976

Montipora sinuosa Pillai \& Scheer

Montipora solanderi (Ellis \& Solander)

Montipora spongiosa (Ehrenberg 1834)

Montipora spongodes Bernard 1897

Montipora spumosa (Lamarck 1816)

Montipora stellata Bemard 1897

Montipora studeri Vaughan 1907

Montipora stylosa (Ehrenberg 1834)

Montipora sulcata Crossland

Montipora sumilonensis Nemenzo

Montipora suvadivae Pillai \& Scheer

Montipora tenuicaulis Vaughan 1907

Montipora tenuissima Bernard 1897

Montipora trabeculata Bernard 1897

Montipora tuberculosa (Lamarck 1816)

Montipora tuberosa Bernard

Montipora turgescens Bernard 1897

Montipora turtlensis Veron \& Wallace 1984

Montipora undata Bernard 1897

Montipora vaughani Hoffmeister 1925 


\title{
Alveopora Blainville 1830
}

II

(Red Sea [64], East and South Africa [8, 43]. Indian Ocean north to the Maldives and Andaman and Nicobar Islands [59]; south to Madagascar and south-west tip of Australia [80]. South-East Asia. Pacific Ocean, north to Japan [40], Midway Islands and Hawaiian Islands; south to Lord Howe Island and Kermadec Islands [75]; east to Tuamotu Archipelago [42])

Countries listed without reference numbers are within the distribution range shown in Veron [75]

American Samoa [50]; Australia; British Indian Ocean Territory [65, 95]; Brunei; Christmas Island; Comoros; Cook Islands; Djibouti; Egypt [64]; Ethiopia; Federated States of Micronesia; Fiji; French Polynesia [30, 42]; Guam; Hawaiian Islands; Hong Kong [64a]; India [58, 59]; Indonesia; Israel [64]; Jordan [64]; Japan [78a]; Kenya [43]; Kiribati; Madagascar [56, 57]; Malaysia [4]: including Sabah [101]; Maldives [20, 65]; Marshall Islands; Mauritius; Mozambique [8]; Myanmar; Nauru; New Caledonia [102]; Niue; Northern Marianas; Palau; Papua New Guinea; Philippines [79]; Reunion; Samoa; Saudi Arabia [1b]; Seychelles [65, 99]; Singapore; Solomon Islands; Somalia; Sri Lanka; Sudan [64]; Tanzania [43]; Taiwan [34]; Thailand [36, 72]; Tokelau; Tonga; Tuvalu; Vanuatu [76]; Wallis and Futuna; Yemen

In general a fairly sparsely distributed, uncommon coral, although may be locally common.

Twenty-seven nominal species, an estimated 16 valid species, of which 8 are recognised from Australia [75]. 12 reasonably well established species are listed below

\author{
Alveopora allingi Hoffmeister 1925 \\ Alveopora catalai Wells 1968 \\ Alveopora daedalea (Forskảl 1775) \\ Alveopora excelsa Verrill 1863 \\ Alveopora explanata Hoffmeister 1945 \\ Alveopora fenestrata (Lamarck 1816) \\ Alveopora gigas Veron 1985 \\ Alveopora japonica Eguchi 1968 \\ Alveopora marionensis Veron \& Pichon 1982 \\ Alveopora ocellata Wells 1954 \\ Alveopora octoformis Blainville \\ Alveopora retipora Blainville \\ Alveopora retusa Verrill 1864 \\ Alveopora spongiosa Dana 1846 \\ Alveopora superficialis Pillai \& Scheer \\ Alveopora tizardi Bassett-Smith 1890 \\ Alveopora verrilliana Dana 1872 \\ Alveopora viridis (Quoy \& Gaimard 1827)
}

Goniopora Blainville 1830

II

(Red Sea [64], Persian Gulf, East and South Africa [8, 43]. Indian Ocean north to the Arabian Gulf [9], Gulf of Kutch (north-west India), Andaman and Nicobar Islands [59] and Mergui Archipelago; south to Madagascar and south-west tip of Australia [80]. South-East Asia. Pacific Ocean, north to Japan [40]; south to Lord Howe Island, east to Marshall Islands and Tuamotu Archipelago $[42,75]$ )

Countries listed without reference numbers are within the distribution range shown in Veron [75]

American Samoa [50]; Australia; Bahrain [9. 67]; British Indian Ocean Territory [65, 95]; Brunei; China [111, 112]; Christmas Island; Comoros; Cook Islands; Djibouti; Egypt [64]; Ethiopia; Federated States of Micronesia; Fiji; French Polynesia [30, 42]; Guam; Hong Kong [64a]; India [58, 59]; Indonesia; Iran; Israel [64]; Jordan 
[64]; Japan [78a]; Kenya [43]; Kuwait; Madagascar [56, 57]; Malaysia [4]: including Sabah [101]; Maldives [20, 65]; Marshall Islands; Mauritius; Mozambique [8]; Myanmar; Nauru; New Caledonia [102]; Niue; Northern Marianas; Oman [66]; Palau; Papua New Guinea; Philippines [79]; Qatar; Reunion; Samoa; Saudi Arabia [1b]; Seychelles [65, 99]; Singapore; Solomon Islands; Somalia; Sri Lanka; Sudan [64]; Tanzania [43]; Taiwan [34]; Thailand [36, 72]; Tokelau; Tonga; Tuvalu; United Arab Emirates [9, 67]; Vanuatu [76]; Wallis and Futuna; Yemen

Goniopora is an important and common reef-building coral and often occurs in turbid water. Many species have a widespread distribution, although some are more restricted (e.g. G. pendulus, which is known only from the west coast of Australia [75]). Used as a building material. Thirty-nine nominal species, an unknown number of valid species, but 19 reasonably well established species are listed below. Fourteen species have been recognised from Australia [75]

Goniopora bernardi Faustino 1927

Goniopora burgosi Nemenzo 1955

Goniopora cellulosa Veron 1990

Goniopora columna Dana 1846

Goniopora djiboutiensis Vaughan 1907

Goniopora eclipsensis Veron \& Pichon 1982

Goniopora fruticosa Saville-Kent 1891

Goniopora gracilis (Milne Edwards \& Haime 1860)

Goniopora klunzingeri Marenzeller 1906

Goniopora lobata Milne Edwards \& Haime 1860

Goniopora minor Crossland 1952

Goniopora muscosa Wells 1954

Goniopora norfolkensis Veron \& Pichon 1982

Goniopora palmensis Veron \& Pichon 1982

Goniopora pandoraensis Veron \& Pichon 1982

Goniopora parvastella Ortman 1888

Goniopora pedunculata Blainville 1830

Goniopora pendulus Veron 1985

Goniopora planulata (Ehrenberg 1834)

Goniopora polyformis Zou 1980

Goniopora pulvinula Wells 1954

Goniopora reptans Bernard

Goniopora samoa Bernard 1903

Goniopora savignyi Dana 1846

Goniopora somaliensis Vaughan 1907

Goniopora stokesi Milne Edwards \& Haime 1851

Goniopora stutchburyi Wells 1955

Goniopora tenella (Quelch 1886)

Goniopora tenuidens Quelch 1886

Goniopora traceyi Wells 1954

Goniopora viridis Quoy \& Gaimard

\section{Porites Link 1807}

II

(Caribbean [61, 96] to Bermuda [68, 100]; south to Brazil [49]. Sao Thome, Cape Verde Islands, Gulf of Guinea and Angola [70]. Red Sea 24], Persian Gulf, East and South Africa [8, 43]. Indian Ocean north to Arabian Gulf [9], Gulf of Kutch (north-west India), Andaman and Nicobar Islands [59] and Mergui Archipelago; south to Madagascar [56], Cocos-Keeling Islands [87] and south-west tip of Australia [80]. South-east Asia. Pacific Ocean, north to southern Japan [40], Midway Islands and Hawaiian 1slands [52]; south to Lord Howe 1sland, Kermadec Islands [75]; east to California [69], Galapagos Islands Islands [38] and Easter Island [90])

Countries listed without reference numbers are within the distribution range shown in Smith [68], Veron [75] or Wood [100]

American Samoa [50]; Angola [27]; Anguilla; Antigua and Barbuda; Australia; Bahamas [47]; Bahrain [9,16]; 
Barbados; Belize [11]; Bermuda [47]; Brazil [49]; British Indian Ocean Territory [65, 95]; British Virgin Islands [37]; Brunei; Cape Verde [27]; Cayman Islands; Chile (Easter Island) [90]; Christmas Island; China [111, 112]; Cocos (Keeling) Islands [77]; Colombia [62]; Comoros; Cook Islands; Costa Rica [33]; Cuba [48]; Djibouti; Dominica; Dominican Republic; Ecuador [8]; Egypt [64]; Ethiopia; Federated States of Micronesia; Fiji; French Polynesia [30, 42]; Grenada; Guadeloupe; Guam; Haiti; Hawaiian Islands [16, 52]; Honduras; Hong Kong [64a]; India [58, 59]; Indonesia; Iran; Israel [64]; Jamaica [96]; Japan [78a]; Jordan [64]; Kenya [43]; Kiribati; Kuwait; Madagascar [56, 57]; Malaysia [4]: including Sabah [101]; Maldives [20, 65]; Marshall Islands; Martinique; Mauritius; Mexico [47]; Montserrat; Mozambique [8]; Myanmar; Nauru; Netherlands Antilles [47]; New Caledonia [102]; New Zealand: Kermadec Islands; Nicaragua; Niue; Northern Marianas; Oman [66]; Palau; Panama [61]; Papua New Guinea; Philippines [79]; Pitcaim Islands [54]; Puerto Rico; Qatar; Reunion; Saint Kitts and Nevis; Saint Lucia; Saint Vincent and the Grenadines; Samoa; Sao Tome and Principe [27]; Saudi Arabia [1b]; Seychelles [65, 99]; Singapore; Solomon Islands; Somalia; Sri Lanka; Sudan [64]; Tanzania [43]; Taiwan [34]; Thailand [36, 72]; Tokelau; Tonga; Trinidad and Tobago; Turks and Caicos; Tuvalu; United Arab Emirates [9, 67]; USA: California [26], Florida [47]; Vanuatu [76]; Virgin Islands of the United States; Venezuela; Wallis and Futuna; Yemen

Synaraea is a subgenus of Porites, although has been given generic status by some authors. Porites is one of the most important reef-building genera, often forming very large colonies. It occurs widely elsewhere, and is one of the most tolerant of all corals to turbid water

Used as a building material

Approximately 122 nominal species, the majority of which are invalid [75]. The number of valid species is unknown; 16 have been recorded from Australia [75] and probably 5 occur in the Atlantic [100]. 37 reasonably well established species are listed below.

Porites annae Crossland 1952

Porites aranetai Nemenzo 1955

Porites astreoides Lamarck 1816

Porites attenuata Nemenzo 1955

Porites australiensis Vaughan 1918

Porites baracoensis Vaughan 1919

Porites bernardi Vaughan 1907

Porites branneri Rathbun 1888

Porites brighami Vaughan 1907

Poriles clavaria Lamarck

Porites cocosensis Wells 1950

Porites colonensis Zlatarski 1990

Porites columnaris KLunzinger

Porites compressa Dana 1846

Porites convexa Verrill

Porites cribripora Dana 1846

Porites cumulatus Nemenzo 1955

Porites cylindrica Dana 1846

Porites danae (Verrill)

Porites deformis Nemenzo 1955

Porites densa Vaughan 1918

Porites divaricata LeSueur 1820

Porites duerdeni Vaughan 1907

Porites echinulata Klunzinger 1879

Porites eridani Umbgrove 1940

Porites erosa Dana 1848

Porites evermanni Vaughan 1907

Porites exilis Gardiner 1898

Porites exserta Pillai

Porites faustinoi (Hoffmeister 1925)

Porites favosa Dana 1846

Porites furcata Lamarck 1816 
Porites gaimardi Milne Edwards \& Haime

Porites galeata Nemenzo

Porites hawaiiensis Vaughan 1907

Porites heronensis Veron 1985

Porites horizontalata (Hoffmeister 1925)

Porites irregularis (Verrill 1864)

Porites latistella Quelch 1886

Porites lichen Dana 1846

Porites limosa Dana 1846

Porites lobata Dana 1846

Porites lutea Milne Edwards \& Haime 1860/51?

Porites mannarensis Pillai

Porites matthaii Wells 1954

Porites mauritiensis Bernard

Porites mayeri Vaughan 1918

Porites minicoiensis Pillai

Porites mordax Dana 1846

Porites mucronata Dana

Porites murravensis Vaughan 1918

Porites myrmidonensis Veron 1985

Porites negrosensis Veron 1990

Porites nigrescens Dana 1848

Porites nodifera Klunzinger 1879

Porites nodulosa Verrill

Porites okinawensis Veron 1990

Porites palmata Dana 1846

Porites parvistellata Quelch

Porites paschalensis Vaughan 1906

Porites porites (Pallas 1766)

Porites portoricensis (Vaughan 1919)

Porites profundus Rehberg

Porites pukoensis Vaughan 1907

Porites punctata (Klunzinger)

Porites purpurea Gardiner 1898

Porites reticulosa Dana 1846

Porites rus (Forskảl 1775)

Porites saccharata Brüggemann

Porites sillimaniani Nemenzo 1976

Porites solida (Forskảl 1775)

Porites somaliensis Gravier 1911

Porites stephensoni Crossland 1952

Porites studeri Vaughan 1907

Porites superflua Gardiner 1898

Porites sverdrupi Durham 1947

Porites tenuis Verrill 1866

Porites thurstoni Pillai

Porites trimurata Gardiner 1898

Porites umbellifera Gardiner 1898

Porites vaughani Crossland 1952

Porites violettae Nemenzo

Porites waylandi Foster 1986 
Stylaraea Milne Edwards \& Haime I851

II

(Red Sea, East Africa, Madagascar, Aldabra, southern Philippines, Palau Islands, Guam and Caroline Islands south to Lesser Sunda Islands, New Guinea, New Britain and the Great Barrier Reef [75])

Countries listed without reference numbers are within the distribution range shown in Veron [75]

Australia; Djibouti; Egypt; Ethiopia; Federated States of Micronesia; Indonesia; Israel; Kenya; Madagascar; Mozambique [8]; Palau; Papua New Guinea; Philippines [79]; Seychelles: Aldabra; Sudan; Tanzania

A single species $S$. punctata, first described as Porites punctata, and subsequently considered a subgenus of Porites. Very rare, and restricted to shallow-water environments uninhabited by other corals [75]

\section{Stylaraea punctata (Linnaeus 1758)}

Family SIDERASTREIDAE Vaughan \& Wells 1943

Anomastraea von Marenzeller 1901

(Persian Gulf and Arabian Gulf [9]. East and South Africa [8, 43])

Kenya [8]; Mozambique [43]; Oman [66]; Tanzania [8]; United Arab Emirates [9]

A small, uncommon coral.

I species [100]

Anomastraea irregularis von Marenzeller 1901

Coscinaraea Milne Edwards \& Haime 1848

II

(Red Sea [64], Persian Gulf, East and South Africa [8, 43]. Indian Ocean, north to Arabian Gulf [9], Gulf of Kutch (north-west India), Gulf of Mannar (southern India) [59], Mergui Archipelago; south to Madagascar and east to Australia, including the entire south coast [75]. South-east Asia. Pacific Ocean, north to Japan [40], Midway Islands and Hawaiian Islands [52], south to south-east Australia; east to the Tuamotu Archipelago [42])

Countries listed without reference numbers are within the distribution range shown in Veron [75]

American Samoa [50]; Australia; Bahrain [9. 67]; British Indian Ocean Territory [65, 95]; Brunei; Christmas Island; Comoros; Cook Islands; Djibouti; Egypt [64]; Ethiopia; Federated States of Micronesia; Fiji; French Polynesia [30, 42]; Guam; Hawaiian Islands [52]; Hong Kong [64a]; India [58, 59]; Indonesia; Iran; Israel [64]; Jordan [64]; Japan [78a]; Kenya [43]; Kiribati; Kuwait; Madagascar [56, 57]; Malaysia [4, 101]; Maldives [20, 65]; Marshall Islands; Mauritius; Mozambique [8]; Myanmar; Nauru; New Caledonia [102]; Niue; Northern Marianas; Oman [66]; Palau; Papua New Guinea; Philippines [79]; Qatar; Reunion; Samoa; Saudi Arabia [1b]; Seychelles [65, 99]; Singapore; Solomon Islands; Somalia; Sri Lanka; Sudan [64]; Tanzania [43]; Taiwan [34]; Thailand [36, 72]; Tokelau; Tonga; Tuvalu; United Arab Emirates [9, 67]; Vanuatu [76]; Wallis and Futuna; Yemen

Coscinaraea is unusual in that two species (C. mcneilli and $C$. marshae) are restricted to southern temperate waters and never found on tropical coral reefs. Most species are relatively uncommon, except for $C$. exesa [75] Approximately 14 nominal species. Eight valid species, of which 6 occur in Australia [75]

Coscinaraea bottae Milne Edwards \& Haime 1848

Coscinaraea columna (Dana 1846)

Coscinaraea crassa Veron \& Pichon 1980

Coscinaraea exesa (Dana I846)

Coscinaraea hahazimaensis Yabe \& Sugiyama 1936

Coscinaraea marshae Wells 1962

Coscinaraea mcneilli Wells 1962

Coscinaraea monile (Forskål 1775)

Coscinaraea wellsi Veron \& Pichon 1980 
Craterastrea Head 1983

British Indian Ocean Territory [65]; Sudan; Egypt [39]

1 species

Craterastrea levis Head 1983 EG, IO, SD

Horastrea Pichon 1971

(East Africa; Madagascar and central Indian Ocean [75])

Madagascar; Mauritius [65]; Mozambique [65]; Reunion [65]

An inconspicuous coral, although relatively common within its range [75]

I species

Horastrea indica Pichon 1971

Maeandroseris Rousseau 1854

II

I species

Maeandroseris bottae Rousseau 1854

Plesioseris Duncan 1884

11

?3 species

Plesioseris australiae (Rousseau 1854)

\section{Psammocora Dana 1846}

(Red Sea [64], Persian Gulf, East and South Africa [8, 43]. Indian Ocean, north to Arabian Gulf [9], southern India, Andaman and Nicobar Islands [59] and Mergui Archipelago; south to Madagascar [56], Cocos-Keeling Islands [87] and Houtman Abrolhos Islands (western Australia) [80]. South-east Asia. Pacific Ocean, north to southern Japan [40], Midway Islands and Hawaiian Islands [52]; south to Lord Howe Island and Pitcairn Islands Island [75]; east to California, Easter Island, Galapagos Islands [38] and Colombia)

Countries listed without reference numbers are within the distribution range shown in Veron [75]

American Samoa [50]; Australia; Bahrain [9. 67]; British Indian Ocean Territory [65, 95]; Brunei; Chile (Easter Island) [38]; Christmas Island; Cocos (Keeling) Islands [77]; Colombia [62]; Comoros; Cook Islands; Costa Rica [33]; Djibouti; Ecuador (Galapagos Islands) [38]; Egypt [64]; Ethiopia; Fiji; Federated States of Micronesia; French Polynesia [30, 42]; Guam; Hawaiian Islands [52]; Hong Kong [64a]; India [58, 59]; Indonesia; Iran; Israel [64]; Japan [78a]; Jordan [64]; Kenya [43]; Kiribati; Kuwait; Madagascar [56, 57]; Malaysia [4]: including Sabah [101]; Maldives [20, 65]; Marshall Islands; Mauritius; Mozambique [8]; Myanmar; Nauru; New Caledonia [102]; Niue; Northern Marianas; Oman [66]; Palau; Papua New Guinea; Philippines [79]; Pitcairn Islands [54]; Qatar; Reunion; Samoa; Saudi Arabia [1b]; Seychelles [65, 99]; Singapore; Solomon Islands; Somalia; Sri Lanka; Sudan [64]; Tanzania [43]; Taiwan [34]; Thailand [36, 72]; Tokelau; Tonga; Tuvalu; United Arab Emirates [9, 67]; Vanuatu [76]; Wallis and Futuna; Yemen

The genus has previously been included in the Family Thamnasteriidae, but a recent revision [75] places Psammocora close to Coscinaraea in the Family Siderastreidae. A relatively common, widespread reef coral, although some of the species are rare. Twenty-seven nominal species, an unknown number of valid species. 13 reasonably well established species are listed below. Seven have been recognised from Australia [75].

Psammocora brighami Vaughan 1907

Psammocora contigua (Esper 1795)

Psammocora decussata Yabe \& Sugiyama 1937

Psammocora digitata Milne Edwards \& Haime 1851

Psammocora explanulata van der Horst 1922 
Psammocora folium Umbgrove 1947

Psammocora haimeana Milne Edwards \& Haime 1851

Psammocora nierstraszi van der Horst 1921

Psammocora obtusangula (Lamarck 1816)

Psammocora profundacella Gardiner 1898

Psammocora samoaensis Hoffmeister 1925

Psammocora savigniensis Gardiner 1898

Psammocora stellata Verrill 1864

Psammocora superficialis Gardiner 1898

Psammocora vaughani Yabe \& Sugiyama 1936

Psammocora verrilli Vaughan 1907

Pseudosiderastrea Yabe \& Sugiyama 1935

(Eastern Indian Ocean; Gulf of Kutch (north-west India), southern India, Andaman and Nicobar Islands [59].

Malaysia, Indonesia, Irian Jaya, Philippines north to Taiwan, south to Dampier (north-west Australia) and Great

Barrier Reef [75])

Countries listed without reference numbers are within the distribution range shown in Veron [75]

Australia; Brunei; Christmas Island; India [58, 59]; Indonesia; Malaysia [4]; Myanmar; Oman [66]; Papua New Guinea; Philippines [79]; Singapore; Thailand [36, 72]; Vanuatu [76]

A small, inconspicuous and uncommon reef coral [30, 41]. Three nominal species, one valid species [75].

Pseudosiderastrea tayam(a)i Yabe \& Sugiyama 1935

Siderastrea Blainville 1830

11

(Caribbean [61, 96], south to Brazil [49]. Bermuda [25, 41]. Gulf of Guinea, Cape Verde Islands and Senegal [70]. Red Sea [64], Persian Gulf, Arabian Gulf [9], East and South Africa [8, 43], India and Myanmar[65])

Countries listed without reference numbers are within the distribution range shown in Smith [68], Veron [75] or Wood [100]

Anguilla; Antigua and Barbuda; Bahamas [47]; Bahrain [9,16]; Barbados; Belize [11]; Bermuda [47]; Brazil [49]; British Virgin Islands [37]; Cape Verde [27]; Colombia [62]; Costa Rica [33]; Cuba [48]; Djibouti; Dominica; Dominican Republic; Egypt [64]; Ethiopia; Grenada; Guadeloupe; Haiti; Honduras; lndia [58, 59]; Iran; Israel [64]; Jamaica [96]; Jordan [64]; Kenya [43]; Kuwait; Madagascar [56, 57]; Martinique; Mauritius; Mexico [47]; Montserrat; Mozambique [8]; Myanmar; Netherlands Antilles [47]; Nicaragua; Oman [66]; Panama [61]; Puerto Rico; Saint Kitts and Nevis; Saint Lucia; Saint Vincent and the Grenadines; Sao Tome and Principe [27]; Saudi Arabia [1b]; Senegal [27]; Seychelles; Somalia; Sri Lanka; Sudan [64]; Tanzania [43]; Trinidad and Tobago; Turks and Caicos; United Arab Emirates [9, 67]; USA(Florida) [47]; Virgin Islands of the United States; Venezuela; Yemen

In the western Atlantic, Siderastrea is common and occurs on most reefs [100]. Elsewhere in its range it is inconspicuous and uncommon. Three species occur in the Western Atlantic, of which one, S. stellata is endemic to Brazil [49]. There is probably only a single species in the Red Sea and western Indian Ocean, S. savignyana

Siderastrea radians (Pallas 1766)

Siderastrea savignyana Milne Edwards \& Haime 1849

Siderastrea siderea (Ellis \& Solander 1786)

Siderastrea stellata Verrill 1868 
Agaricia Lamarck 1801

(Caribbean [61, 96] south to Brazil [49]; Bermuda [68, 100])

Countries listed without reference numbers are within the distribution shown in Smith [68] and Wood [100]

Anguilla; Antigua and Barbuda; Bahamas [47]; Barbados; Belize [11]; Bermuda [47]; Brazil [49]; British Virgin Islands [37]; Cayman Islands; Colombia [62]; Costa Rica [33]; Cuba [48]; Dominica; Dominican Republic; Grenada; Guadeloupe; Haiti; Honduras; Jamaica [96]; Martinique; Mexico [47]; Montserrat; Netherlands Antilles [47]; Nicaragua; Panama [61]; Puerto Rico; Saint Kitts and Nevis; Saint Lucia; Saint Vincent and the Grenadines; Trinidad and Tobago; Turks and Caicos; USA(Florida) [47]; Virgin Islands of the United States; Venezuela

A common coral occuring in most reef habitats; grows on deep reefs in low light conditions. Probably 7 species

Agaricia agaricites (Linnaeus 1758)

Agaricia fragilis Dana 1848

Agaricia grahamae Wells 1973

Agaricia humilis Verrill 1901

Agaricia lamarcki Milne Edwards \& Haime 1851

Agaricia tenuifolia Dana 1848

Agaricia undata (Ellis \& Solander 1786)

Coeloseris Vaughan 1918

11

(Andaman and Nicobar Islands [59]. South-east Asia, north to Ryukyu Islands, south to Rowley Shoals (northern Australia) and Middleton Reef (eastern Australia); east to Samoa [75])

Countries listed without reference numbers are within the distribution range shown in Veron [75]

Australia; Brunei; Federated States of Micronesia; Fiji; lndia [58, 59]; Indonesia; Japan [78a]; Malaysia [4]: including Sabah [101]; Myanmar; New Caledonia [102]; Palau; Papua New Guinea; Philippines [79]; Samoa; Solomon Islands; Taiwan [34]; Thailand [36, 72]; Tokelau; Tonga; Vanuatu [76]; Wallis and Futuna

May be locally common, especially on the reef rim, but generally the species has a scattered distribution [75, 100]. Three nominal species, one valid species

\section{Coeloseris mayeri Vaughan 1918}

\section{Gandineroseris Scheer \& Pillai 1974}

\section{II}

(Red Sea [64], East Africa. Indian Ocean, north to the Maldives [75], Andaman and Nicobar Islands [59] and Mergui Archipelago; south to Madagascar and Ningaloo Reefs (western Australia). South-east Asia. Pacific Ocean, north to southern Japan, Guam, Phoenix Islands [75], Tubuai Islands [42]; south to Solitary Islands (eastern Australia) and Tonga; east to Galapagos Islands [75])

Countries listed without reference numbers are within the distribution range shown in Veron [75]

American Samoa [50]; Australia; British Indian Ocean Territory [65, 95]; Brunei; Christmas Island; Cocos (Keeling) Islands [77]; Colombia (Pacific) [62]; Comoros; Cook Islands; Djibouti; Egypt [64]; Ethiopia; Federated States of Micronesia; Fiji; French Polynesia [30, 42]; Guam; India [58, 59]; Indonesia; Iran; Israel [64]; Jordan [64]; Japan [78a]; Kenya [43]; Kiribati; Madagascar [56, 57]; Malaysia [4, 101]; Maldives [20, 65]; Marshall Islands; Mauritius; Myanmar; Nauru; New Caledonia [102]; Niue; Oman [66]; Palau; Papua New Guinea; Philippines [79]; Reunion; Samoa; Saudi Arabia [1b]; Seychelles [65, 99]; Singapore; Solomon Islands; Sudan [64]; Tanzania [43]; Taiwan [34]; Thailand [36, 72]; Tokelau; Tonga; Tuvalu; Vanuatu [76]; Wallis and Futuna; Yemen One widely distributed species; possibly a second, undescribed, from Thailand [75] 
Leptoseris Milne Edwards \& Haime 1849

I1

(Caribbean [100]. Red Sea [64], East and South Africa [8, 43]. Indian Ocean, north to the Maldives [95], Andaman and Nicobar Islands [59] and Mergui Archipelago; south to Madagascar and Houtman Abrolhos lslands (western Australia) [80]. Pacific Ocean, north to southern Japan [71], Midway Islands and Hawaiian Islands [52]; south to Lord Howe Island, Kermadec Islands [75]; east to Panama, Colombia, Galapagos Islands [38] and Easter lsland [90])

Countries listed without reference numbers are within the distribution range shown in Smith [68], Veron [75] or Wood [100]

American Samoa [50]; Anguilla; Antigua and Barbuda; Australia; Bahamas [47]; Barbados; Belize [11]; Brazi] [49]; British Indian Ocean Territory [65, 95]; British Virgin Islands [37]; Brunei; Cayman Islands; Chile (Galapagos Islands) [38]; China [111, 112]; Christmas Island; Cocos (Keeling) Islands [77]; Colombia [62]; Comoros; Cook Islands; Costa Rica [33]; Cuba [48]; Djibouti; Dominica; Dominican Republic; Ecuador; Egypt [64]; Ethiopia; Federated States of Micronesia; Fiji; French Polynesia [30, 42]; Grenada; Guadeloupe; Guam; Haiti; Hawaiian Islands [52]; Honduras; Hong Kong [64a]; India [58, 59]; Indonesia; Israel [64]; Jamaica [96]; Japan [78a]; Jordan [64]; Kenya [43]; Kiribati; Madagascar [56, 57]; Malaysia [4]: including [101]; Maldives [20, 65]; Marshall Islands; Martinique; Mauritius; Mexico [47]; Montserrat; Mozambique [8]; Myanmar; Nauru; Netherlands Antilles [47]; New Caledonia [102]; New Zealand: Kermedec Islands; Nicaragua; Niue; Northern Marianas; Oman [66]; Palau; Panama [61]; Papua New Guinea; Philippines [79]; Pitcairn Islands [54]; Puerto Rico; Reunion; Saint Kitts and Nevis; Saint Lucia; Saint Vincent and the Grenadines; Samoa; Saudi Arabia [1 b]; Seychelles [65, 99]; Singapore; Solomon Islands; Somalia; Sri Lanka; Sudan [64]; Tanzania [43]; Taiwan [34]; Thailand [36, 72]; Tokelau; Tonga; Trinidad and Tobago; Turks and Caicos; Tuvalu; USA: California, Florida; Vanuatu [76]; Virgin Islands of the United States; Venezuela; Wallis and Futuna; Yemen

The presence of this genus in the western Atlantic has only fairly recently been recognised [35]. Prior to this, the two species were referred to as Helioseris cucullata and Agaricia cailleti. Leptoseris forms delicate, often leafy colonies, particularly on lower reef slopes and walls. Collected for use as a curio. Twenty-five nominal species; probably 14 valid species (listed below), of which 8 are known from Australia [75]. Only 2 species occur in the western Atlantic [35]

\section{Leptoseris amitoriensis Veron 1990}

Leptoseris cailleti (Duchassaing \& Michelotti 1864)

Leptoseris cucullata (Ellis \& Solander 1786)

Leptoseris edwardsi Rousseau 1854

Leptoseris explanata Yabe \& Sugiyama 1941

Leptoseris foliosa Dinesen 1980

Leptoseris fragilis Milne Edwards \& Haime 1849

Leptoseris gardineri van der Horst 1921

Leptoseris glabra Dinesen 1980

Leptoseris hawaiiensis Vaughan 1907

Leptoseris incrustans (Quelch 1886)

Leptoseris mycetoseroides Wells 1954

Leptoseris papyracea (Dana 1846)

Leptoseris scabra Vaughan 1907

Leptoseris solida (Quelch 1886)

Leptoseris tenuis van der Horst 1921

Leptoseris yabei (Pillai \& Scheer 1976)

Pachyseris Milne Edwards \& Haime 1849

II

(Red Sea [64], East and South Africa [8, 43]. Indian Ocean, north to Lacadives, Gulf of Kutch (north-west India), Andaman and Nicobar lslands [59] and Mergui Archipelago; south to Madagascar and Houtman Abrolhos Islands (western Australia) [80]. South-east Asia. Pacific Ocean, north to southem Japan [71], Guam, Marshall Islands [88] and Line Islands; south to Great Barrier Reef [75]; east to Marquesas and Tuamotu Archipelago [42]) 
Countries listed without reference numbers are within the distribution range shown in Veron [75]

American Samoa [50]; Australia; British Indian Ocean Territory [65, 95]; Brunei; China [111, 112]; Christmas Island; Cocos (Keeling) Islands [77]; Comoros; Cook Islands; Djibouti; Egypt [64]; Ethiopia; Federated States of Micronesia; Fiji; French Polynesia [30, 42]; Guam; India [58, 59]; Indonesia; Israel [64]; Jordan [64]; Japan [78a]; Kenya [43]; Kiribati; Madagascar [56, 57]; Malaysia [4]: including Sabah [101]; Maldives [20, 65]; Marshall Islands; Mauritius; Mozambique [8]; Myanmar; Nauru; New Caledonia [102]; Niue; Palau; Papua New Guinea; Philippines [79]; Reunion; Samoa; Saudi Arabia [1b]; Seychelles [65, 99]; Singapore; Solomon Islands; Somalia; Sri Lanka; Sudan [64]; Tanzania [43]; Taiwan [34]; Thailand [36, 72]; Tokelau; Tonga; Tuvalu; Vanuatu [76]; Wallis and Futuna; Yemen

A relatively common reef coral, occurring in a range of reef habitats. Collected for use as a curio. Twelve nominal species; of which 4 are currently recognised as valid.

Pachyseris carinata Brüggemann 1879

Pachyseris foliosa Veron 1990

Pachyseris gemmae Nemenzo 1955

Pachyseris rugosa (Lamarck 1801)

Pachyseris speciosa (Dana 1846)

Pavona Lamarck 1801

11

(Red Sea [64], Persian Gulf, East and South Africa [8, 43]. Indian Ocean, north to Arabian Gulf [9], Gulf of Mannar (southern India), Andaman and Nicobar Islands [59] and Mergui Archipelago; south to Madagascar [56], Cocos-Keeling Islands [87] and Houtman Abrolhos lslands (western Australia) [80]. South-east Asia. Pacific Ocean north to southern Japan [40], Midway Islands, Hawaiian lslands [52]; south to Lord Howe and Pitcairn Islands Islands; east to California [69], Galapagos Islands and Colombia [38])

Countries listed without reference numbers are within the distribution range shown in Veron [75]

American Samoa [50]; Australia; Bahrain [9. 67]; British Indian Ocean Territory [65, 95]; Brunei; China [111, 112]; Christmas Island; Cocos (Keeling) Islands [77]; Colombia [62]; Comoros; Cook Islands; Costa Rica [33]; Djibouti; Ecuador (Galapagos 1slands) [38]; Egypt [64]; Ethiopia; Federated States of Micronesia; Fiji; French Polynesia [30, 42]; Guam; Hong Kong [64a]; India [58, 59]; Indonesia; Iran; 1srael [64]; Jordan [64]; Japan [78a]; Kenya [43]; Kiribati; Kuwait; Madagascar [56, 57]; Malaysia [4]: including Sabah [101]; Maldives [20, 65]; Marshall Islands; Mauritius; Mozambique [8]; Myanmar; Nauru; New Caledonia [102]; Niue; Northern Marianas; Oman [66]; Palau; Papua New Guinea; Philippines [79]; Pitcaim Islands [54]; Qatar; Reunion; Samoa; Saudi Arabia [1b]; Seychelles [65, 99]; Singapore; Solomon Islands; Somalia; Sri Lanka; Sudan [64]; Tanzania [43]; Taiwan [34]; Thailand [36, 72]; Tokelau; Tonga; Tuvalu; United Arab Emirates [9, 67]; USA: California [69]; Vanuatu [76]; Wallis and Futuna; Yemen

Pavona is a fairly common coral that occurs in most reef habitats. Collected for use as a curio. Approximately 50 nominal species, but there is great variability in the genus and there are probably only around 15 valid species, of which 8 are known from Australia [75]

Pavona acuticarinata (Umbgrove)

Pavona bipartita Nemenzo 1980

Pavona cactus (Forskảl 1775)

Pavona calicifera Gardiner 1898

Pavona clavus (Dana 1846)

Pavona clivosa (Verrill 1869)

Pavona complanata (Verrill 1866)

Pavona danai Milne Edwards \& Haime 1860

Pavona decussata (Dana 1846)

Pavona diffluens Lamarck 1816

Pavona diminuta Veron 1990

Pavona divaricata Lamarck 1816

Pavona duerdeni Vaughan 1907 
Pavona explanulata Lamarck 1816

Pavona foliosa (Verrill 1866)

Pavona formosa (Dana 1846)

Pavona frondifera (Lamarck 1816)

Pavona galapagensis Durham \& Barnard

Pavona gigantea Verrill 1869

Pavona intermedia Gardiner 1898

Pavona maldivensis (Gardiner 1905)

Pavona minor Brüggemann

Pavona minuta Wells 1954

Pavona repens Brüggemann 1878

Pavona varians Verrill 1864

Pavona venosa (Ehrenberg 1834)

Pavona xarifae Scheer \& Pillai 1974

Pavona yamanarii (Yabe \& Sugiyama 1933)

Family MICRABACIIDAE Vaughan 1905

Lepropenus Moseley 1881

(Worldwide, including Antarctica. 871-5,000m [23])

6 species are known, of which 5 are named [23].

Leptopenus antarcticus Cairns 1989

Leptopenus discus Moseley 1881

Leptopenus hypocoelus Moseley 1881

Leptopenus irinae Keller 1977

Leptopenus solidus Keller 1977

Letepsammia Yabe \& Eguchi 1932

(Indo-West Pacific. 128-828m)

2 species are known; one undescribed [23].

Letepsammia formosissima (Moseley 1876)

Micrabacia Milne Edwards \& Haime 1849

?2 species

Rhombopsammia Owens 1986

11

(China Sea and Philippine Sea. 424-1,401m)

2 species [23]

Rhombopsammia niphada Owens 1986

Rhombopsammia squiresi Owens 1986

Stephanophyllia Michelin 1841

(Indo-West Pacific. 15-635m)

4 species [23]

Stephanophyllia elegans (Bronn 1837)

Stephanophyllia complicata Moseley 1876

Stephanophyllia fungulus Alcock 1902

Stephanophyllia neglecta Boschma 1923 
Family FUNGIACYATHIDAE Chevalier 1987

Fungiacyathus Sars 1872

(Cosmopolitan, including Antarctica [23])

II

Solitary; free living. 99-6,328m. Until recently, included in the Family Fungiidae. Unknown number of species (possibly about 20); a worldwide revision of Fungiacyathus is required $[10,23]$. 14 species are listed below.

Fungiacyathus aleuticus Keller 1976

Fungiacyathus crispus (Pourtalès 1871)

Fungiacyathus dennanti Cairns \& Parker?

Fungiacyathus durus Keller 1976

Fungiacyathus fissilis Cairns 1984

Fungiacyathus fragilis Sars 1872

Fungiacyathus granulosus Cairns 1989

Fungiacyathus hydra Zibrowius \& Gili 1990

Fungiacyathus marenzelleri (Vaughan 1906)

Fungiacyathus paliferus (Alcock 1902)

Fungiacyathus pliciseptus Keller 1976

Fungiacyathus pseudostephanus Keller 1976

Fungiacyathus pusillus (Pourtalès 1868)

Fungiacyathus sibogae (Alcock 1902)

Fungiacyathus stabilis (Gardiner \& Waugh 1939)

Fungiacyathus stephanus (Alcock 1893)

Fungiacyathus symmetricus (Pourtalès 1871)

Fungiacyathus turbinolioides Cairns 1989

Fungiacyathus variegatus Cairns 1989

Family FUNGIIDAE Dana 1846

Cantharellus Hoeksma 1989

See Cycloseris

\section{Ctenactis Verrill 1864}

11

(Red Sea, Indian Ocean, South-East Asia, Australia to mid-Pacific Islands [75])

Countries listed without reference numbers are within the distribution range shown in Veron [75]

American Samoa [50]; Australia; Brunei; Myanmar; Cook Islands; Djibouti; Egypt [64]; Ethiopia; Federated States of Micronesia; Fiji; French Polynesia [30, 42]; Guam; India [58, 59]; Indonesia; Israel [64]; Jordan [64]; Japan [78a]; Kiribati; Malaysia [4, 101]; Maldives [20, 65]; Marshall Islands; Myanmar; Nauru; New Caledonia [102]; Niue; Palau; Papua New Guinea; Philippines [79]; Samoa; Saudi Arabia [1 b]; Singapore; Solomon Islands [44]; Sudan [64]; Thailand [36, 72]; Tokelau; Tonga; Tuvalu; Wallis and Futuna; Yemen

The species now referred to as Fungia (Ctenactis) simplex [75] or Crenactis crassa [44] was previously widely known as Herpetoglossa simplex (Gardiner 1905) [30, 32, 41]

Ctenactis albitentaculata Hoeksema 1989

Ctenactis crassa (Dana 1846)

Ctenactis echinata (Pallas 1766) 
Cycloseris Milne Edwards \& Haime 1849

(Red Sea [64], Persian Gulf, East and South Africa [43, 44]. Indian Ocean, north to the Gulf of Kutch (north-west India), southern India, Andaman and Nicobar Islands [59] and the Mergui Archipelago; south to Madagascar, and Houtman Abrolhos Islands (western Australia) [75]. South-east Asia. Pacific Ocean, north to southern Japan [71] and Hawaiian Islands 16]; south to Lord Howe Island and Pitcaim Jslands Island [75]; east to California [69], Galapagos Islands [38], Colombia and Easter Island [90])

Countries listed without reference numbers are within the distribution range shown in Veron [75]

American Samoa [50]; Australia; Bahrain [9. 67]; British Indian Ocean Territory [65, 95]; Brunei; Chile (Easter Island) [90]; China [111, 112]; Christmas Island; Comoros; Cook Islands; Djibouti; Ecuador (Galapagos Islands) [38]; Egypt [64]; Ethiopia; Federated States of Micronesia; Fiji; French Polynesia [30, 42]; Guam; Hawaiian Islands [52]; India [58, 59]; Indonesia; Iran; Israel [64]; Jordan [64]; Japan [78a]; Kenya [43]; Kiribati; Kuwait; Madagascar [56, 57]; Malaysia [4]: including Sabah [101]; Maldives [20, 65]; Marshall Islands; Mauritius; Mozambique [8]; Myanmar; Nauru; New Caledonia [102]; Niue; Northern Marianas; Palau; Papua New Guinea; Philippines [79]; Pitcairn Islands [54]; Qatar; Reunion; Samoa; Saudi Arabia [1b]; Seychelles [65, 99]; Singapore; Solomon Islands; Somalia; Sri Lanka; Sudan [64]; Tanzania [43]; Taiwan [34]; Thailand [36, 72]; Tokelau; Tonga; Tuvalu; United Arab Emirates [9, 67]; Vanuatu [76]; Wallis and Futuna; Yemen

Cycloseris is a free-living coral and relatively rare, with the exception of $C$. cyclolites which is widely distributed and common on soft substrata on and around reefs. Collected for use as a curio.

A recent revision of the Family Fungiidae places Cycloseris as a subgenus of Fungia [44], and also includes Diaseris in the same subgenus. Ten species are recognised in this subgenus. The revision places two species (Cycloseris doederleini and $C$. noumeae) in a new genus, Cantharellus [45]. These revisions have not been accepted by other coral taxonomists [e.g. 65, 80, 78a] and for the listings below Cycloseris and Diaseris are therefore still listed as separate genera and the Cantharellus species are placed back in Cycloseris. In addition, C. erosa, $C$. patelliformis and $C$. marginata are retained in the list although considered in the revision [45] to be junior synonyms of other Cycloseris species

14 currently accepted species are listed below, 7 of which are recognised from Australia [75]

Cycloseris costulata (Ortmann 1889)

Cycloseris curvata (Hoeksema 1989)

Cycloseris cyclolites (Lamarck 1801)

Cycloseris doederleini (von Marenzeller 1907)

Cycloseris erosa (Döderlein 1901)

Cycloseris hexagonalis (Milne Edwards \& Haime 1848)

Cycloseris marginata (Boschma 1923)

Cycloseris noumeae (Hoeksema \& Best 1984)

Cycloseris patelliformis (Boschma 1923)

Cycloseris sinensis Milne Edwards \& Haime 1851

Cycloseris somervillei (Gardiner 1909)

Cycloseris tenuis (Dana 1846)

Cycloseris vaughani (Boschma 1923)

Diaseris Milne Edwards \& Haime 1849

(Red Sea, East Africa. Indian Ocean, north to Lakshadweep and Thailand [44], south to Madagascar, Cocos-Keeling Islands and Houtman Abrolhos Islands (western Australia) [75]. South-east Asia. Pacific Ocean, north to southern Japan, Northern Marianas and Hawaiian Islands; south to Great Barrier Reef, Tonga and Tuamotu Archiplego; east to California, Columbia and Galapagos Islands [75])

Countries listed without reference numbers are within the distribution range shown in Veron [75]

American Samoa [50]; Australia; British Indian Ocean Territory [65, 95]; Brunei; China [111, 112]; Christmas Island; Comoros; Cook Islands; Djibout1; Egypt [64]; Ethiopia; Federated States of Micronesia; Fiji; French Polynesia [30, 42]; Guam; Hawaiian Islands [52]; India [58, 59]; Indonesia; Israel [64]; Jordan [64]; Japan 
[78a]; Kenya [43]; Kiribati; Malaysia [4, 101]; Maldives [20, 65]; Marshall Islands; Mauritius; Mozambiquc [8]; Myanmar; Nauru; New Caledonia [102]; Niue; Northern Marianas; Palau; Papua New Guinea; Philippines [79]; Reunion; Samoa; Saudi Arabia [1b]; Seychelles [65, 99]; Singapore; Solomon Islands; Somalia; Sri Lanka; Sudan [64]; Tanzania [43]; Taiwan [34]; Thailand [36, 72]; Tokelau; Tonga; Tuvalu; USA: California; Wallis and Futuna

Diaseris is a free-living coral restricted to soft substrata on reefs, and sometimes locally common. Four nominal species; at least 2 valid species [78a]. There is disagreement on the generic status of Diaseris. It has been placed in the genus Cycloseris [64] and as the subgenus Cycloseris of Fungia [44], but is considered by Veron to be a distinct genus [78a].

Diaseris distorta (Michelin 1843)

Diaseris fragilis (Alcock 1893)

Fungia Lamarck 1801

II

(Red Sea [64], East and South Africa [8, 43]. Indian Ocean, north to Lacadives and Andaman and Nicobar Islands [59] and Mergui Archipelago; south to Madagascar [56], Cocos-Keeling 1slands [87] and north-western Australia [80]. South-east Asia. Pacific Ocean, north to Midway Islands and Hawaiian Islands [52]; south to Great Barrier Reef [75], east to Line Islands, Marquesas [42] and Pitcaim Islands Island [75])

Countries listed without reference numbers are within the distribution range shown in Veron [75]

American Samoa [50]; Australia; British Indian Ocean Territory [65, 95]; Brunei; China [111, 112]; Christmas Island; Cocos (Keeling) Islands [77]; Comoros; Cook 1slands; Djibouti; Egypt [64]; Ethiopia; Federated States of Micronesia; Fiji; French Polynesia [30, 42]; Guam; Hawaiian Islands [52]; Hong Kong [64a]; India [58, 59]; Indonesia; Israel [64]; Jordan [64]; Japan [78a]; Kenya [43]; Kiribati; Madagascar [56, 57]; Malaysia [4]: including Sabah [101]; Maldives [20, 65]; Marshall Islands; Mauritius; Mozambique [8]; Myanmar; Nauru; New Caledonia [102]; Niue; Northem Marianas; Palau; Papua New Guinea; Philippines [79]; Pitcairn Islands [54]; Reunion; Samoa; Saudi Arabia [1b]; Seychelles [65, 99]; Singapore; Solomon Islands; Somalia; Sri Lanka; Sudan [64]; Tanzania [43]; Taiwan [34]; Thailand [36, 72]; Tokelau; Tonga; Tuvalu; Vanuatu [76]; Wallis and Futuna; Yemen

Fungia is widely distributed on and around reefs. A few species are rare (e.g. F. scabra), but the m.jority are common $[30,41]$. Widely collected for use as a curio.

Five subgenera are generally recognised, Pleuractis, Ctenactis, Verillofungia, Danafungia and Fungia [30, 36], Approximately 65 nominal species have been described for these subgenera, but probably only 25 are valid species, of which 15 occur in Australia [75]. In a recent review of the fungiids [44], three additional subgenera are recognised (Cycloseris, Wellsofungia and Lobactis) while Ctenactis is elevated to generic status. In this arrangement, 23 valid Fungia species are recognised [44]. Currently, the elevation of Ctenactis to generic level has been generally accepted, but the other re-arrangements have not. Therefore for this listing the taxonomic status as described by Veron [82] is maintained.

18 species belonging to the subgenera Pleuractis, Verillofungia, Danafungia and Fungia are listed:

Fungia concinna Verrill 1864

Fungia corona Döderlein 1901

Fungia danai (Milne Edwards \& Haime 1851)

Fungia fralinae Nemenzo 1955

Fungia fungites (Linnaeus 1758)

Fungia granulosa Klunzinger 1879

Fungia gravis Nemenzo 1955

Fungia horrida Dana 1846

Fungia klunzingeri Dōderlein 1901

Fungia moluccensis van der Horst 1919

Fungia paumotensis Stutchbury 1833

Fungia repanda Dana 1846 
Fungia scabra Dôderlein 1901

Fungia scruposa Klunzinger 1879

Fungia scutaria Lamarck 1801

Fungia spinifer Claereboudt \& Hoeksema 1987

Fungia taiwanensis Hoeksma \& Dai 1991

Fungia valida Verrill 1864

Halomitra Dana 1846

II

(East Africa [4j]]. Indian Ocean, north to Maldives [95] and Thailand [44]; south to Madagascar and Chagos Archipelago [64]. South-east Asia. Pacific Ocean, north to Ryukyu Islands, Guam and Marshall Islands; south to Great Barrier Reef, New Caledonia and Tonga [75]; east to Line Islands and Tuamotu Archipelago [42])

Countries listed without reference numbers are within the distribution range shown in Veron [75]

American Samoa; Australia; British Indian Ocean Territory [65, 95]; Brunei; Comoros; Federated States of Micronesia; Fiji; Guam; Indonesia; Kenya [43]; Kiribati; Madagascar [56, 57]; Malaysia [4]: including [101]; Maldives [20,65]; Marshall Islands; Mauritius; Myanmar; Nauru; New Caledonia [102]; Palau; Papua New Guinea; Philippines [79]; Reunion; Samoa; Seychelles [65, 99]; Singapore; Solomon 1slands; Tanzania [43]; Thailand [36, 72]; Tokelau; Tonga; Tuvalu; Vanuatu [76]; Wallis and Futuna

Free living corals; relatively uncommon. Collected for use as a curio. Six nominal species, possibly 2 valid species. $H$. pileus occurs throughout the range of the genus; $H$. clavator is known only from the Philippines and Indonesia

Halomitra clavator Hoeksma 1989

Halomitra pileus (Linnaeus 1758)

Heliofungia Wells 1966

II

(Ryukyu Islands, south to Philippines, Bomeo, Java, northern Australia and Great Barrier Reef; east to Caroline Islands, Solomon Islands and New Caledonia [13, 30, 42])

Countries listed without reference numbers are within the distribution range shown in Veron [75]

Australia; Federated States of Micronesia; Indonesia; Japan [78a]; Malaysia: Sabah [101]; Nauru; New Caledonia [102]; Palau; Papua New Guinea; Philippines [79]; Singapore; Solomon Islands; Vanuatu [76]

Collected for use as a curio, also as an aquarium specimen. The name Heliofungia was introduced to describe Fungia actiniformis [89], but was used until recently only as a subgenus. It is now accepted that this coral is distinct and warrants generic status $[13,30,32]$

1 species

Heliofungia actiniformis (Quoy \& Gaimard 1833)

Herpolitha Eschscholtz 1825

II

(Red Sea [64], East and South Africa [8, 43]. Indian Ocean, north to Maldives 39], Andaman and Nicobar Islands [59] and Thailand [44]; south to Madagascar, Cocos-Keeling Islands [87] and Ningaloo Reefs (western Australia) [80]. South-east Asia. Pacific Ocean, north to Ryukyu Islands, Ogaswara-gunto Islands, Northern Marianas and Marshall Islands; south to Great Barrier Reef, New Caledonia and Tonga; east to Line Islands and Tuarnotu Archipelago [11, 30])

Countries listed without reference numbers are within the distribution range shown in Veron [75]

American Samoa [50]; Australia; British Indian Ocean Territory [65, 95]; Brunei; Christmas Island; Cocos (Keeling) 1slands [77]; Cook Islands; Djibouti; Egypt [64]; Ethiopia; Federated States of Micronesia; Fiji; French Polynesia [30, 42]; India [58, 59]; Indonesia; Israel [64]; Jordan [64]; Japan [78a]; Kenya [43]; Madagascar [56, 57]; Malaysia [4]: including Sabah [101]; Maldives [20, 65]; Marshall Islands; Mauritius; 
Mozambique [8]; Myanmar; Nauru; New Caledonia [102]; Niue; Palau; Papua New Guinea; Philippines [79]; Reunion; Samoa; Saudi Arabia [1b]; Seychelles [65, 99]; Singapore; Solomon Islands; Somalia; Sri Lanka; Sudan [64]; Tanzania [43]; Taiwan [34]; Thailand [36, 72]; Tokelau; Tonga; Tuvalu; Vanuatu [76]; Wallis and Futuna; Yemen

Free-living corals. Collected for use as a curio.

Nine nominal species; probably 2 valid species

Herpolitha limax (Esper 1797)

Herpolitha weberi (van der Horst 1921)

Lithophyllon Rehberg 1892

\section{II}

(Mergui Archipelago, south to Ningaloo Reefs (western Australia) [80]. South-east Asia. Pacific Ocean, north to southern Japan [40] and Marshall Islands; south to Great Barrier Reef; east to Samoa [75])

Countries listed without reference numbers are within the distribution range shown in Veron [75]

American Samoa [50]; Australia; Brunei; Christmas Island; Federated States of Micronesia; Fiji; Hong Kong [64a]; Indonesia; Japan [78a]; Malaysia: Sabah [101]; Myanmar; New Caledonia [102]; Palau; Papua New Guinea; Philippines [79]; Samoa; Singapore; Solomon Islands; Thailand [36, 72]; Vanuatu [76]; Wallis and Futuna

An attached, colonial coral. Found on reef slopes, but always uncommon

Seven nominal species, possibly 5 valid species

Lithophyllon edwardsi (Rousseau 1854)

Lithophyllon levistei Nemenzo 1971

Lithophyllon lobata (van der Horst 1921)

Lithophyllon mokai Hoeksema 1989

Lithophyllon undulatum Rehberg 1892

Podabacia Milne Edwards \& Haime 1849

II

(Red Sea [64], East and South Africa [8, 43]. Indian Ocean, north to Lacadives and Sri Lanka [59] and Mergil Archipelago; south to Madagascar [56] and Ningaloo Reefs (western Australia). South-east Asia. Pacific Ocean, north to southern Japan and Northern Marianas; south to Great Barrier Reef [75] and New Caledonia [102]; east to Tuamotu Archipelago [75])

Countries listed without reference numbers are within the distribution range shown in Veron [75]

American Samoa [50]; Australia; British Indian Ocean Territory [65, 95]; Brunei; China [111, 112]; Christmas Island; Comoros; Cook Islands; Djibouti; Egypt [64]; Ethiopia; Federated States of Micronesia; Fiji; French Polynesia [30, 42]; Guam; Hong Kong [64a]; India [58, 59]; Indonesia; Israel [64]; Jordan [64]; Japan [78a]; Kenya [43]; Madagascar [56, 57]; Malaysia [4]: including Sabah [101]; Maldives [20, 65]; Mauritius; Mozambique [8]; Myanmar; Naun; New Caledonia [102]; Niue; Northern Marianas; Palau; Papua New Guinea; Philippines [79]; Reunion; Samoa; Saudi Arabia [1b]; Seychelles [65, 99]; Singapore; Solomon 1slands; Somalia; Sri Lanka; Sudan [64]; Tanzania [43]; Taiwan [34]; Thailand [36, 72]; Tokelau; Tuvalu; Vanuatu [76]; Wallis and Futuna; Yemen

An attached fungiid, found in most reef habitats, but relatively uncommon. Collected for use as a curio.

Two valid species

Podabacia crustacea (Pallas 1766)

Podabacia motuporensis Veron 1990 
Polyphyllia Quoy \& Gaimard 1833/0?

II

(East Africa [44]. Indian Ocean, north to Maldives [95], Andaman and Nicobar Islands [59] and Mergui Archipelago; south to Madagascar and Ningaloo Reefs (western Australia) [80]. South-east Asia. Pacific Ocean, north to Ryukyu Islands and Northern Marianas; south to Great Barrier Reef and New Caledonia; east to Samoa and Tonga [75])

Countries listed without reference numbers are within the distribution range shown in Veron [75]

Australia; British Indian Ocean Territory [65, 95]; Brunej; China [111, 112]; Christmas Island; Federated States of Micronesia; Fiji; Guam; India [58, 59]; Indonesia; Japan [78a]; Madagascar [56, 57]; Malaysia [4]: including Sabah [101]; Maldives [20, 65]; Marshall Islands; Mauritius; Myanmar; Nauru; New Caledonia [102]; New Zealand; Niue; Northem Marianas; Palau; Papua New Guinea; Philippines [79]; Reunion; Samoa; Seychelles [65, 99]; Singapore; Solomon Islands; Somalia; Sri Lanka; Tanzania [43]; Taiwan [34]; Thailand [36, 72]; Tokelau; Tonga; Tuvalu; Vanuatu [76]; Wallis and Futuna

Free-living and often occurring with Fungia. Collected for use as a curio. Eleven nominal species; 2 or 3 three species $[13,30]$, of which $P$. talpina is the commonest

Polyphyllia novaehiberniae (Lesson 1831)

Polyphyllia talpina (Lamarck 1801)

\section{Sandalolitha Quelch 1884}

(Maldives, Mergui Archipelago, south to Ningaloo Reefs (western Australia) [30, 31]. South-east Asia. Pacific Ocean, north to Kyushu Islands and Marshall Islands [88]; south to Great Barrier Reef, New Caledonia and Fiji; east to Line Islands [75])

Countries listed without reference numbers are within the distribution range shown in Veron [75]

Australia; Bahrain [9. 67]; Brunei; Christmas Island; Cocos (Keeling) Islands [77]; Federated States of Micronesia; French Polynesia [30, 42]; Guam; Indonesia; Japan [78a]; Kiribati; Malaysia [4]: including Sabah [101]; Maldives [20, 65]; Marshall Islands; Myanmar; Nauru; New Caledonia [102]; Northern Marianas; Palau; Papua New Guinea; Philippines [79]; Pitcaim Islands [54]; Singapore; Solomon Islands; Taiwan [34]; Thailand [36, 72]; Tuvalu; Vanuatu [76]

Free-living and relatively common throughout the range of the genus. Parahalomitra is synonymous. Four nominal species, 2 valid species $[44,75]$.

Sandalolitha dentata Quelch 1884

Sandalolitha robusta (Quelch 1886)

\section{Zoopilus Dana 1846/8?}

Il

(Malaysia, eastern Indonesia. Pacific Ocean, north to Ryukyu Islands, south to New Guinea; east to Marshall Islands and Fiji [75])

Countries listed without reference numbers are within the distribution range shown in Veron [75]

Federated States of Micronesia; Indonesia; Fiji; Japan [78a]; Malaysia: Sabah [101]; Marshall Islands; Mauritius; Nauru; Palau; Papua New Guinea; Philippines [79]; Solomon Islands; Vanuatu [76]

Free-living on soft substrata on and around reefs; an uncommon coral. I species

Zoopilus echinatus Dana 1846 


\section{Family RHIZANGIIDAE d'Orbigny 1851}

This Family is listed by Chevalier (1987) [32] as Family Astrangiidae Milne Edwards \& Haime 1857, but is retained as Family Rhizangiidae by Cairns 1991 [25]. Rhizangiid corals are probably widely distributed, but are small and under-recorded. Country distributions are not given.

Astrangia Milne Edwards \& Haime 1848

II

(Widespread in many seas [55]. Caribbean [61, 96], western Atlantic to Brazil [49, 107] and Indo-Pacific, including California $[69,75])$

Solitary corals which occur in shallow water, mostly in caves or on vertical faces on rocks or reefs [75]. Thirty or more nominal species, but the genus has never been properly reviewed [55]. An unknown number of species; the 9 listed below are probably valid

Astrangia browni Palmer 1928

Astrangia dentasa Verrill 1866

Astrangia equatorialis Durham \& Barnard 1952

Astrangia lajollaensis Durham 1947

Astrangia macrodentala Thiel 1940

Astrangia poculata (Ellis \& Solander 1786)

Astrangia rathbuni Vaughan 1906

Astrangia solitaria (Lesueur 1817)

Astrangia woodsi Wells 1955

Cladangia Milne Edwards \& Haime 1851

(Indo-Pacific)

India [59]

Possibly 2 species

Cladangia exusta Lütken?

Coenangia Verrill 1869

Possibly 2 species

Coenangia conferta Verrill 1869

Colangia Pourtalès 1871

(Caribbean [61, 96])

Possibly 2 species

Colangia immersa Pourtalès 1871

Culicia Dana 1846

I1

(Red Sea [64], Indo-Pacific, including temperate zones such as New Zealand [63])

Small corals which occur in shallow water, mostly in caves or on vertical faces on rocks or reefs [75]. About 12 nominal species, of which possibly 6 are valid [28].

Culicia australiensis Hoffmeister 1933

Culicia cuticulata Kluzinger

Culicia hoffmeisteri Squires 1966

Culicia japonica Yabe \& Eguchi 1936

Culicia rubeola (Quoy \& Gaimard 1833)

Culicia smithi Milne Edwards \& Haime 1850

Culicia stellata Dana 1846 


\section{Culicia tenella Dana 1846}

Culicia truncata Dana 1846

Culicia verreauxi Milne Edwards \& Haime 1850

Oulangia Milne Edwards \& Haime 1848

(Indo-Pacific)

Japan [71]; Galapagos Islands

An unknown number of species

Oulangia bradleyi Verrill 1866

Oulangia stokesiana (Milne Edwards \& Haime 1848)

Phyllangia Milne Edwards \& Haime 1848

Antarctica [12], Caribbean [61, 96], Mediterranean, Atlantic Ocean [107], Brazil [49], Gulf of Guinea [49], Red Sea [64], Pacific including Gulf of California [69]. About 8-100 m depth

Phyllangia is considered by some [25] to belong in the Family Caryophylliidae, but is traditionally included in the Astrangiidae. 10 nominal species. Approximately 8 recent species

Phyllangia americana Milne Edwards \& Haime 1849

Phyllangia consagensis (Durham \& Barnard 1952)

Phyllangia dispersa Verrill 1864

Phyllangia granulata W. Koch 1886

Phyllangia hayamaensis (Eguchi 1968)

Phyllangia mouchezii (Lacaze-Duthiers 1897)

Phyllangia papuensis Studer 1878

Polycyathus Duncan 1876

II

Was moved to the Family Rhizangiidae by Verheij \& Best (1987) 173], but this move from the Family Caryophylliidae has not been accepted by other workers e.g. Cairns 199I [25]

Family OCULINIDAE Gray 1847

Acrhelia Milne Edwards \& Haime 1849

II

(Sabah (East Malaysia) [101]; north to Ryukyu Islands, Northern Marianas and Marshall Islands; south to Great Barrier Reef and New Caledonia; east to Vanuatu [75])

Countries listed without reference numbers are within the distribution range shown in Veron [75]

American Samoa [50]; Australia; Federated States of Micronesia; Guam; Indonesia; Japan [78a]; Malaysia: Sabah [101]; Marshall Islands; Nauru; New Caledonia [102]; Northern Marianas; Palau; Papua New Guinea; Philippines [79]; Solomon Islands; Tonga; Vanuatu [76]; Wallis and Futuna; Yemen

A delicate, uncommon reef coral $[75,100]$

1 species

Acrhelia horrescens (Dana 1846)

Amphelia Milne Edwards \& Haime 1849

About 12 species

Amphelia oculata (Linnaeus 1758) 
Archohelia Vaughan 1919

(Known only from the Great Barrier Reef, Australia [75])

A single species, occurring in shallow water [75]

Archohelia rediviva Wells \& Alderslade 1979

Bathelia Moseley 1881

(Southern South America. 500-1,250m depth)

Bathelia candida Moseley 1881

Cyathelia Milne Edwards \& Haime 1849

(Indo-Pacific)

Probably two species; one is recognised from Australia [75]

Cyathelia axillaris (Ellis \& Solander 1786)

Galarea Oken 1815

(Red Sea [64], East and South Africa [8, 43]. Indian Ocean, north to Lakshadweep, Gulf of Mannar (southem India) and Mergui Archipelago; south to Madagascar [56] and Houtman Abrolhos 1slands (western Australia) [80]. South-east Asia. Pacific Ocean, north to southern Japan; south to Great Barrier Reef [75]; east to Line Islands and Tuamotu Archipelago [42])

Countries listed without reference numbers are within the General distribution range shown in Veron [75]

American Samoa [50]; Australia; British Indian Ocean Territory [65, 95]; Brunei; China [111, 112]; Christmas Island; Comoros; Cook Islands; Djibouti; Egypt [64]; Ethiopia; Federated States of Micronesia; Fiji; French Polynesia [30, 42]; Guam; Hong Kong [64a]; India [58, 59]; Indonesia; 1srael [64]; Jordan [64]; Japan [78a]; Kenya [43]; Kiribati; Madagascar [56, 57]; Malaysia [4]: ircluding Sabah [101]; Maldives [20, 65]; Marshall Islands; Mauritius; Mozambique [8]; Myanmar; Naunu; New Caledonia [102]; Niue; Northern Marianas; Oman [66]; Palau; Papua New Guinea; Philippines [79]; Reunion; Samoa; Saudi Arabia [1b]; Seychelles [05, 99]; Singapore; Solomon Islands; Somalia; Sri Lanka; Sudan [64]; Tanzania [43]; Taiwan [34]; Thailand [36, 72]; Tokelau; Tonga; Tuvalu; Vanuatu [76]; Wallis and Futuna; Yemen

The most frequently encountered is G. fascicularis, which is very common in a wide range of reef habitats. Collected for use as a curio. Twenty-four nominal species, probably 5 valid species, 2 in Australia [75].

Galaxea alta Nemenzo ?

Galaxea astreata (Lamarck 1816)

Galaxea clavus (Dana 1846)

Galaxea fascicularis (Linnaeus 1758)

Galaxea lauensis Hoffmeister 1945

Galaxea paucisepta Claereboudt 1990 
An unknown number of species; three are recognised from Australia [75]. 80-1,500m depth

Madrepora arbuscula (Moseley 1881)

Madrepora candida (Moseley 1881)

Madrepora carolina (Pourtalès 1871)

Madrepora exigua (Pourtalès 1871)

Madrepora investigatoris (Alack 1898)

Madrepora galapagensis Vaughan 1906

Madrepora kauaiensis Vaughan 1907

Madrepora tenuis (Moseley 1881)

Neohelia Moseley 1881

1 species

Neohelia porcellana (Moseley 1881)

Oculina Lamarck 1816

11

(Mediterranean, Western Atlantic [41, 46], West Africa [70], New Zealand [63], Galapagos Islands [28])

Countries listed without reference numbers are within the distribution range shown in Smith [68] and Wood [100]

Anguilla; Antigua and Barbuda; Bahamas [47]; Barbados; Belize [11]; Bermuda [47]; Brazil [49]; British Virgin Islands [37]; Cayman Islands; Colombia; Costa Rica [33]; Cuba [48]; Dominica; Dominican Republic; Ecuador (Galapagos Islands) [28]; Grenada; Guadeloupe; Haiti; Honduras; Jamaica [96]; Liberia [27]; Martinique; Mexico [47]; Montserrat; Netherlands Antilles [47]; New Zealand [63]; Nicaragua; Panama [61]; Puerto Rico; Saint Kitts and Nevis; Saint Lucia; Saint Vincent and the Grenadines; Sao Tome \& Principe [27]; Trinidad and Tobago; Turks and Caicos; USA(Florida) [47]; Virgin Islands of the United States; Venezuela

Approximately 20 nominal species. Nine listed below are reasonably well established. The common shallow water species found in the Caribbean is $O$. diffusa; other species occur in deeper water [100].

Oculina arbuscula L. Agassiz 1864

Oculina banksii Milne Edwards \& Haime

Oculina diffusa Lamarck 1816

Oculina patagonica De Angelis 1908

Oculina profunda Cairns 1991

Oculina robusta Pourtalès 1871

Oculina tenella Pourtalès 1871

Oculina valenciennesi?

Oculina varicosa Lesueur 1821

Oculina virgosa Squires 1958

Schizoculina Wells 1937

?2 species

Schizoculina fissispina (Milne Edwards \& Haime 1850)

Sclerhelia Milne Edwards \& Haime 1850

(Indo-Pacific)

5 nominal species

Sclerhelia hirella (Pallas 1766) 
(Red Sea [64], East and South Africa [8, 43]. Indian Ocean, north to Arabian Gulf [9], Lacadives, southern India and Mergui Archipelago; south to Madagascar and Houtman Abrolhos Islands (western Australia) [75]. South-east Asia. Pacific Ocean, north to southern Japan [40] and Marshall Islands; south to Lord Howe Island; east to Tuamotu Archipelago [42])

Countries listed without reference numbers are within the distribution range shown in Veron [75]

American Samoa [50]; Australia; British Indian Ocean Territory [65, 95]; Brunei; Christmas Island; Comoros; Cook Islands; Djibouti; Egypt [64]; Ethiopia; Federated States of Micronesia; Fiji; French Polynesia [30, 42]; Guam; Hong Kong [64a]; India [58, 59]; Indonesia; Israel [64]; Jordan [64]; Japan [78a]; Kenya [43]; Kiribati; Madagascar [56, 57]; Malaysia [4]: including Sabah [101]; Maldives [20, 65]; Marshall 1slands; Mauritius; Mozambique [8]; Myanmar; Nauru; New Caledonia [102]; Niue; Northern Marianas; Oman [66]; Palau; Papua New Guinea; Philippines [79]; Reunion; Samoa; Saudi Arabia [1b]; Seychelles [65, 99]; Singapore; Solomon Islands; Somalia; Sri Lanka; Sudan [64]; Tanzania [43]; Taiwan [34]; Thailand [36, 72]; Tokelau; Tonga; Tuvalu; Vanuatu [76]; Wallis and Futuna; Yemen

Found in a wide range of reef habitats and fairly common. Collected for use as a curio. Probably 7 valid species

Echinophyllia aspera (Ellis \& Solander 1786)

Echinophyllia echinata (Saville-Kent 1871)

Echinophyllia echinoporoides Veron \& Pichon 1980

Echinophyllia hirsuta Nemenzo

Echinophyllia maxima Moll \& Best 1984

Echinophyllia nishihirai Veron 1990

Echinophyllia orpheensis Veron \& Pichon 1980

Echinophyllia patula (Hodgson \& Ross 1982)

Echinophyllia subglabra Nemenzo

\section{Mycedium Oken 1815}

(Red Sea [64], East and South Africa [8, 43]. Indian Ocean, north to Maldives, southem India, Andaman and Nicobar Islands [59] and Mergui Archipelago; south to Madagascar and Houtman Abrolhos Islands (western Australia) [80]. South-east Asia. Pacific Ocean north to southern Japan [71] and Marshall Islands; south to Great Barrier Reef and Lord Howe Island [75]; east to Tubuai Islands [42])

Countries listed without reference numbers are within the distribution range shown in Veron [75]

American Samoa [50]; Australia; British Indian Ocean Territory [65, 95]; Brunei; Christmas Island; Cocos (Keeling) Islands [77]; Comoros; Cook Islands; Djibouti; Egypt [64]; Ethiopia; Federated States of Micronesia; Fiji; French Polynesia [30, 42]; Guam; India [58, 59]; Indonesia; Israel [64]; Jordan [64]; Japan [78a]; Kenya [43]; Kiribati; Madagascar [56, 57]; Malaysia [4]: including Sabah [101]; Maldives [20, 65]; Marshall 1slands; Mauritius; Mozambique [8]; Myanmar; Naunu; New Caledonia [102]; Niue; Oman [66]; Palau; Papua New Guinea; Philippines [79]; Reunion; Samoa; Saudi Arabia [1b]; Seychelles [65, 99]; Singapore; Solomon Islands; Somalia; Sri Lanka; Sudan [64]; Tanzania [43]; Taiwan [34]; Thailand [36, 72]; Tokelau; Tonga; Tuvalu; Vanuatu [76]; Wallis and Futuna; Yemen

Collected for use as a curio. Three nominal species; 2 valid species 
(Red Sea [64], East and South Africa [8, 43]. Indian Ocean, north to Lacadives, southern India and Mergui Archipelago; south to Madagascar and Houtman Abrolhos 1slands (western Australia) [75]. South-east Asia. Pacific Ocean, north to southern Japan and Marshall Islands; south to Elizabeth and Middleton reefs (E. Australia) [75]; east to Tubuai 1slands [42])

Countries listed without reference numbers are within the distribution range shown in Veron [75]

American Samos [50]; Australia; British Indian Ocean Territory [65, 95]; Brunei; Christmas Island; Cocos (Keeling) 1slands [77]; Comoros; Cook Islands; Djibouti; Egypt [64]; Ethiopia; Federated States of Micronesia; Fiji; French Polynesia [30, 42]; Guam; India [58, 59]; Indonesia; Israel [64]; Jordan [64]; Japan [78a]; Kenya [43]; Kiribati; Madagascar [56, 57]; Malaysia [4]: including Sabah [101]; Maldives [20, 65]; Marshall Islands; Mauritius; Mozambique [8]; Myanmar; Nauru; New Caledonia [102]; Niue; Northern Marianas; Oman [66]; Palau; Papua New Guinea; Philippines [79]; Reunion; Samoa; Saudi Arabia [1b]; Seychelles [65, 99]; Singapore; Solomon Islands; Somalia; Sri Lanka; Sudan [64]; Tanzania [43]; Taiwan [34]; Thailand [36, 72]; Tokelau; Tonga; Tuvalu; Vanuatu [76]; Wallis and Futuna; Yemen

Relatively common on ref slopes. Collected for use as a curio. Five nominal species, probably 3 are valid

Oxypora crassispinosa Nemenzo?

Oxypora glabra Nemenzo 1959

Oxypora lacera (Verrill 1864)

Pectinia Oken 1815

II

(East Africa [43]. Indian Ocean, north to Maldives [95], Andaman and Nicobar Islands [59] and Mergui Archipelago; south to Madagascar and Ningaloo Reefs (western Australia). South-east Asia. Pacific Ocean, north to southem Japan (9) and Marshall Islands; south to Great Barrier Reef, New Caledonia and Fiji; east to Line Islands [75])

Countries listed without reference numbers are within the distribution range shown in Veron [75]

Australia; British Indian Ocean Territory [65, 95]; Brunei; Christmas Island; Djibouti; Egypt; Ethiopia; Federated States of Micronesia; Fiji; Guam; India [58, 59]; Indonesia; Japan [78a]; Kenya [43]; Kiribati; Madagascar [56, 57]; Malaysia [4]: including Sabah [101]; Maldives [20, 65]; Marshall Islands; Myanmar; Nauru; New Caledonia [102]; Niue; Palau; Papua New Guinea; Philippines [79]; Seychelles [65, 99]; Singapore; Solomon Islands; Somalia; Sri Lanka; Sudan [64]; Tanzania [43]; Taiwan [34]; Thailand [36, 72]; Vanuatu [76]

The genus is usually well represented on reefs, especially in turbid water. However, some species are rare, for example, P. teres, known only from the Philippines and Australia [75]. Collected for use as a curio. Fourteen nominal species; 4 valid species from Australia [75]

Pectinia alcicornis (Saville-Kent 1871)

Pectinia elongata Rehberg

Pectinia lactuca (Pallas 1766)

Pectinia paeonia (Dana 1846)

Pecrinia symphylloides (Milne Edwards \& Haime)

Pectinia teres Nemenzo \& Montecillo 1981

Physophyllia Duncan 1884

11

(East Africa; Maldives; South-east Asia; southern Japan; south to New Guinea and the Solomon Islands [75])

Countries listed without reference numbers are within the distribution range shown in Veron [75]

Brunei; Federated States of Micronesia; Guam; India [58, 59]; Indonesia; Japan [78a]; Kenya [43]; Malaysia; Myanmar; Palau; Papua New Guinea; Philippines [79]; Seychelles [65, 99]; Solomon 1slands; Tanzania [43]; Thailand $[36,72]$; 
Three nominal species, but possibly only a single valid species. Little is known about this coral [100]. Collected for use as a curio.

\section{Physophyllia ayleni Wells 1934}

\section{Family MUSSIDAE Ortmann 1890}

Acanthastrea Milne Edwards \& Haime 1848

II

(Red Sea [64]. Persian Gulf, East and South Africa [8, 43]. Indian Ocean, north to Arabian Gulf [9], Lacadives, Gulf of Kutch (southern India) [59] and Mergui Archipelago; south to Madagascar and south-west Australia [80]. South-east Asia. Pacific Ocean, north to southern Japan [19]; south to Middleton Reef (south-east Australia) and Lord Howe island [75]; east to Tuamotu Archipelago [42] and Pitcaim Islands Island (75])

Countries listed without reference numbers are within the distribution range shown in Veron [75]

American Samoa [50]; Australia; Bahrain [9. 67]; British Indian Ocean Territory [65, 95]; Brunei; China [111, 112]; Christmas Island; Comoros; Cook Islands; Djibouti; Egypt [64]; Ethiopia; Federated States of Micronesia; Fiji; French Polynesia [30, 42]; Guam; Hong Kong [64a]; India [58, 59]; Indonesia; Iran; Israel [64]; Jordan [64]; Japan [78a]; Kenya [43]; Kiribati; Kuwait; Madagascar [56, 57]; Malaysia [4]: including Sabah [101]; Maldives [20, 65]; Marshall Islands; Mauritius; Mozambique [8]; Myanmar; Nauru; New Caledonia [102]; Niue; Northern Marianas; Oman [66]; Palau; Papua New Guinea; Philippines [79]; Qatar; Reunion; Samoa; Saudi Arabia [1b]; Seychelles [65, 99]; Singapore; Solomon Islands; Somalia; Sri Lanka; Sudan [64]; Tanzania [43]; Taiwan [34]; Thailand [36, 72]; Tokelau; Tonga; Tuvalu; United Arab Emirates [9, 67]; Vanuatu [76]; Wallis and Futuna; Yemen

Generally a fairly common reef coral, occurring in a wide range of habitats $[30,41]$. Collected for use as a building material. Approximately 13 nominal species; probably 9 valid species.

Acanthastrea amakusensis Veron 1990

Acanthastrea bowerbanki Milne Edwards \& Haime 1857

Acanthastrea echinata (Dana 1846)

Acanthastrea hemprichii (Ehrenberg 1934)

Acanthastrea hillae Wells 1955

Acanthastrea ishigakiensis Veron 1990

Acanthastrea lordhowensis Veron \& Pichon 1982

Acanthastrea maxima Sheppard \& Salam 1988

Acanthastrea minuta Moll \& Borel Best 1984

Acanthastrea rotundoflora Chevalier 1975

Acanthastrea simplex Crossland

Acanthophyllia Wells 1937

I species

II

Acanshophyllia deshayesiana (Michelin 1850)

Australomussa Veron 1985

II

Australia (North-west); Myanmar: Mergui Archipelago; Japan; Philippines; Thailand [75]

A rare reef coral. 1 species [74]

Australomussa rowleyensis Veron 1985

Blastomussa Wells 196I

(Red Sea [64]. Indian Ocean, Aldabra north to Arabian Gulf [9] the Maldives and Mergui Archipelago. South-east Asia (excluding east coast of West Malaysia and west coast Borneo). Pacific Ocean, north to Ryukyu Islands, Northern Marianas and Marshall Islands; south to Great Barrier Reef [75], New Caledonia [102] and Fiji; east to Samoa [75]) 
Countries listed without reference numbers are within the distribution range shown in Veron [75]

Australia; British Indian Ocean Territory [65, 95]; China [111, 112]; Christmas Island; Djibouti; Egypt [64]; Ethiopia; Federated States of Micronesia; Fiji; Guam; India [58, 59]; Indonesia; Japan [78a]; Malaysia; Maldives [20, 65]; Marshall Islands; Myanmar; New Caledonia [102]; Niue; Northern Marianas; Oman [66]; Palau; Papua New Guinea; Philippines [79]; Samoa; Saudi Arabia [1b]; Seychelles [65, 99]; Solomon Islands; Sudan [64]; Taiwan [34]; Thailand [36, 72]; Tokelau; Tuvalu; Vanuatu [76]

Generally an uncommon coral, except in some reef slope habitats. Probably 2 valid species.

Blastomussa loyae Head

Blastomussa merleti (Wells 1961)

Blastomussa wellsi Wijsman-Best 1973

\section{Cynarina Brüggemann 1877}

II

(Red Sea [64]. Indian Ocean, north to Maldives [95], southern India and Mergui Archipelago; south to Madagascar [75]. South-east Asia. Pacific Ocean, north to southern Japan [71] and Guam; south to the Great Barrier Reef; east to the Kermadec Islands [75])

Countries listed without reference numbers are within the distribution range shown in Veron [75]

Australia; British Indian Ocean Territory [65, 95]; Brunei; Christmas 1sland; Djibouti; Egypt [64]; Ethiopia; Federated States of Micronesia; Guam; Hong Kong [64a]; India [58, 59]; Indonesia; Japan [78a]; Madagascar [56, 57]; Malaysia [4]: including Sabah [101]; Maldives [20, 65]; Myanmar; New Caledonia [102]; New Zealand: Kermadec Islands; Palau; Papua New Guinea; Philippines [79]; Saudi Arabia [1b]; Seychelles [65, 99]; Singapore; Solomon Islands; Sri Lanka; Sudan [64]; Thailand [36, 72]

A large, solitary reef coral which may be attached or free-living. Uncommon [69, 101]. Nine nominal species, probably only one valid species

Cynarina lacrymalis (Milne Edwards \& Haime 1848)

Indophyllia Gerth 1921

11

Indonesia [2]

A rare coral. 1 species

Indophyllia macassarensis Best \& Hoeksema 1987

Isophyllastrea Matthai 1928

II

(Caribbean $[61,96]$, Bermuda $[68,100]$ )

Countries listed without reference numbers are within the distribution range shown in Smith [68] and Wood [100]

Anguilla; Antigua and Barbuda; Bahamas [47]; Barbados; Belize [11]; Bermuda [47]; British Virgin Islands [37]; Cayman Islands; Colombia [62]; Costa Rica [33]; Cuba [48]; Dominica; Dominican Republic; Grenada; Guadeloupe; Haiti; Honduras; Jamaica [96]; Martinique; Mexico [47]; Montserrat; Netherlands Antilles [47]; Nicaragua; Panama [61]; Puerto Rico; Saint Kitts and Nevis; Saint Lucia; Saint Vincent and the Grenadines; Trinidad and Tobago; Turks and Caicos; USA(Florida) [47]; Virgin Islands of the United States; Venezuela

1 species, generally found in fairly shallow reef habitats [100]

Isophyllastrea rigida (Dana 1848) 
Isophyllia Milne Edwards \& Haime 1851 (Caribbean $[61,96]$, Bermuda $[68,100]$ )

II

Countries listed without reference numbers are within the distribution range shown in Smith [68] and Wood [100]

Anguilla; Antigua and Barbuda; Bahamas [47]; Barbados; Belize [11]; Bermuda [47]; Brazil [49]; British Virgin Islands [37]; Cayman Islands; Colombia [62]; Costa Rica [33]; Cuba [48]; Dominica; Dominican Republic; Grenada; Guadeloupe; Haiti; Honduras; Jamaica [96]; Martinique; Mexico [47]; Montserrat; Netherlands Antilles [47]; Nicaragua; Panama [61]; Puerto Rico; Saint Kitts and Nevis; Saint Lucia; Saint Vincent and the Grenadines; Trinidad and Tobago; Turks and Caicos; USA(Florida) [47]; Virgin Islands of the United States; Venezuela

Two species are recognised; both are fairly common, especially in shallow reef habitats [100]

Isophyllia multiflora Verrill 1901

Isophyllia sinuosa (Ellis \& Solander 1786)

Lobophyllia Blainville 1830

II

(Red Sea [64], East and South Africa [8, 43]. Indian Ocean, north to Lacadives, Andaman and Nicobar Islands [59] and Mergui Archipelago; south to Madagascar [56] and south-west Australia [80]. South-east Asia. Pacific Ocean, north to southern Japan [40], Guam, the Marshall Islands and Line Islands; south to Flinders Reef (eastern Australia) [75]; east to Tuamotu Archipelago [42])

Countries listed without reference numbers are within the distribution range shown in Veron [75]

American Samoa [50]; Australia; British Indian Ocean Territory [65, 95]; Brunei; China [111, 112]; Christmas Island; Cocos (Keeling) Islands [77]; Comoros; Cook Islands; Djibouti; Egypt [64]; Ethiopia; Federated States of Micronesia; Fiji; French Polynesia [30, 42]; Guam; Hong Kong [64a]; India [58, 59]; Indonesia; Israel [64]; Jordan [64]; Japan [78a]; Kenya [43]; Kiribati; Madagascar [56, 57]; Malaysia [4]: including Sabah [101]; Maldives [20, 65]; Marshall Islands; Mauritius; Mozambique [8]; Myanmar; Nauru; New Caledonia [102]; Niue; Palau; Papua New Guinea; Philippines [79]; Reunion; Samoa; Saudi Arabia [1b]; Seychelles [65, 99]; Singapore; Solomon Islands; Somalia; Sri Lanka; Sudan [64]; Tanzania [43]; Taiwan [34]; Thailand [36, 72]; Tokelau; Tonga; Tuvalu; Vanuatu [76]; Wallis and Futuna; Yemen

Most species are relatively common, and are important reef-builders, occurring in a wide range of habitats [100]. L. diminuta is very rare and known only from Swain Reefs (Great Barrier Reef), and L. hataii is also rare although more widely distributed in the Indo-Pacific [75]. Collected for use as a curio, and as a building material. Twenty seven nominal species; 5 valid species [75].

Lobophyllia corymbosa (Forskál 1775)

Lobophyllia costata (Dana 1846)

Lobophyllia diminuta Veron 1985

Lobophyllia hataii Yabe, Sugiyama \& Eguchi 1936

Lobophyilia hemprichii (Ehrenberg 1834)

Lobophyllia pachysepta Chevalier 1975

Lobophyllia robusta Yabe, Sugiyama \& Eguchi 1936

Mussa Oken 1815

(Caribbean [61, 96])

Countries listed without reference numbers are within the distribution range shown in Smith [68] and Wood [100]

Anguilla; Antigua and Barbuda; Bahamas [47]; Barbados; Belize [11]; British Virgin 1slands [37]; Cayman Islands; Colombia [62]; Costa Rica [33]; Cuba [48]; Dominica; Dominican Republic; Grenada; Guadeloupe; Haiti; Honduras; Jamaica [96]; Martinique; Mexico [47]; Montserrat; Netherlands Antilles [47]; Nicaragua; 
Panama [61]; Puerto Rico; Saint Kitts and Nevis; Saint Lucia; Saint Vincent and the Grenadines; Trinidad and Tobago; Turks and Caicos; USA(Florida) [47]; Virgin Islands of the United States; Venezuela

One species, occurs in most reef habitats

Mussa angulosa (Pallas, 1766)

Mussa cactus Dana 1846

Mussa cerebriformis Dana 1846

\section{Mussismilia Ortmann 1890}

Brazil [49]

Three species are recognised, occurring in a range of reef habitats $[49,100]$

Mussismilia braziliensis (Verrill 1868)

Mussismilia hartii (Verrill 1868)

Mussismilia hispida (Verrill 1868)

Mycetophyllia Milne Edwards \& Haime 1848

11

(Caribbean [61, 96])

Countries listed without reference numbers are within the distribution range shown in Smith [68] and Wood [100]

Anguilla; Antigua and Barbuda; Bahamas [47]; Barbados; Belize [11]; British Virgin Islands [37]; Cayman Islands; Colombia [62]; Costa Rica [33]; Cuba [48]; Dominica; Dominican Republic; Grenada; Guadeloupe; Haiti; Honduras; Jamaica [96]; Martinique; Mexico [47]; Montserrat; Netherlands Antilles [47]; Nicaragua; Panama [61]; Puerto Rico; Saint Kitts and Nevis; Saint Lucia; Saint Vincent and the Grenadines; Trinidad and Tobago; Turks and Caicos; USA(Florida) [47]; Virgin Islands of the United States; Venezuela

Generally a widespread and common reef coral [100]. Probably five species.

Mycetophyllia aliciae Wells 1973

Mycetophyllia danana Milne Edwards \& Haime 1849

Mycetophyllia ferox Wells 1973

Mycetophyllia lamarckiana Milne Edwards \& Haime 1848

Mycetophyllia reesi Wells 1973

Scolymia Haime 1852

(Caribbean, south to Brazil [49]. Red Sea (doubtful record) [64] Chagos Archipelago, north to Mergui Archipelago; south to southern coast of Australia. South-east Asia. Pacific Ocean, north to soutbern Japan [71], Guam and the Marshall Islands; south to southeast Australia and Lord Howe Island; east to Pitcaim Islands [75])

Countries listed without reference numbers are within the distribution range shown in Smith [68], Veron [75] or Wood [100]

Anguilla; Antigua and Barbuda; Australia; Bahamas [47]; Barbados; Belize [11]; Brazil [49]; British Indian Ocean Territory [65, 95]; British Virgin Islands; Brunei; Cayman Islands; Christmas Island; Cook Islands; Costa Rica [33]; Cuba [48]; Dominica; Dominican Republic; Federated States of Micronesia; Fiji; French Polynesia [30, 42]; Grenada; Guadeloupe; Guam; Haiti; Honduras; India [58, 59]; Indonesia; Jamaica [96]; Japan [78a]; Madagascar [56, 57]; Malaysia [4]: including Sabah [101]; Maldives [65]; Marshall Islands; Martinique; Mauritius [65]; Mexico [47]; Montserrat; Myanmar; Nauru; Netherlands Antilles [47]; New Caledonia [102]; Nicaragua; Niue; Palau; Panama [61]; Papua New Guinea; Philippines [79]; Pitcaim Islands [54]; Puerto Rico; Reunion [65]; Saint Kitts and Nevis; Saint Lucia; Saint Vincent and the Grenadines; Singapore; Solomon Islands; Taiwan [34]; Thailand [36, 72]; Tonga; Trinidad and Tobago; Turks and Caicos; Tuvalu; Vanuatu [76]; Virgin Islands of the United States; Venezuela; Wallis and Futuna 
Generally an uncommon coral, although it occurs in a wide range of reef and non-reefal habitats $[75,100]$. Scolymia was originally thought to be confined to the westem Atlantic, with Parascolymia its tropical Indo-Pacific homologue. A third genus, Homophyllia, was used to describe a species from southem Australia. Eight nominal species. Two valid species in the Indo-Pacific, probably two in the Caribbean, and a third, $S$. wellsi, endemic to Brazil [49].

Scolymia australis (Milne Edwards \& Haime 1849)

Scolymia cubensis (Milne Edwards \& Haime 1848)

Scolymia lacera (Pallas 1766)

Scolymia vitiensis Brüggemann 1877

Scolymia wellsi Laborel 1967

Symphyllia Milne Edwards \& Haime 1848

Il

(Red Sea [64], East and South Africa [8, 43]. Indian Ocean, north to the Lacadives, Gulf of Kutch (northern India), Andaman and Nicobar Islands [59]; south to Madagascar [56] and south-west tip of Australia [80]. South-east Asia. Pacific Ocean, north to southerm Japan [71] and Marshall 1slands; south to Great Barrier Reef and Tonga; east to Samoa [75])

Countries listed without reference numbers are within the distribution range shown in Veron [75]

American Samoa [50]; Australia; British Indian Ocean Territory [65, 95]; Brunei; China [111, 112]; Christmas Island; Comoros; Djibouti; Egypt [64]; Ethiopia; Federated States of Micronesia; Fiji; Guam; Hong Kong [64a]; India [58, 59]; Indonesia; Japan [78a]; Kenya [43]; Madagascar [56, 57]; Malaysia [4]: including Sabah [101]; Maldives [20, 65]; Marshall Islands; Mauritius; Mozambique [8]; Myanmar; Naurs; New Caledonia [102]; Niue; Northern Marianas; Oman [66]; Palau; Papua New Guinea; Philippines [79]; Reunion; Samoa; Saudi Arabia [1b]; Seychelles [65, 99]; Singapore; Solomon Islands; Somalia; Sri Lanka; Sudan [64]; Tanzania [43]; Taiwan [34]; Thailand [36, 72]; Tokelau; Tonga; Tuvalu; Vanuatu [76]; Wallis and Futuna; Yemen

An important reef-building coral occurring in a wide range of habitats. S. valenciennesii is probably the rarest species, although has a wide geographic distribution $[75,100]$. Collected for use as a building material. Thirteen nominal species [75]; at least 6 valid species

Symphyllia agaricia Milne Edwards \& Haime 1849

Symphyllia erythraea (Klunzinger 1879)

Symphyllia nobilis (Dana 1846)

Symphyllia radians Milne Edwards \& Haime 1849

Symphyllia recta (Dana 1846)

Symphyllia simplex Crossland

Symphyllia valenciennesii Milne Edwards \& Haime 1849

Symphyllia wilsoni Veron 1985

Family MERULINIDAE Verrill 1866

Boninastrea Yabe \& Sugiyama 1935

II

Indonesia [3]; Japan [75]; Taiwan [75]

Little is known of the status and distribution of this coral, which may be an aberrant form of Merulina [75]. 1 species

Boninastrea boninensis Yabe \& Sugiyama 1935

Hydnophora Fischer de Waldheim 1807

II

(Red Sea [64], Persian Gulf, East and South Africa [8, 43]. Indian Ocean, north to Arabian Gulf [9], Gulf of Kutch, southem India, Andaman and Nicobar Islands [59] and Mergui Archipelago; south to Madagascar, Cocos-Keeling Islands [87] and south-westem Australia [80]. South-east Asia. Pacific Ocean, north to southern Japan [40], Northem Marianas and Marshall Islands; south to Elizabeth and Middleton Reefs (eastem Australia) and Lord Howe Island [75]; east to Line Islands and Tuamotu Archipelago [42]) 
American Samoa [50]; Australia; Bahrain [9, 67]; British Indian Ocean Territory [65, 95]; Brunei; China [111, 112]; Christmas Island; Cocos (Keeling) Islands [77]; Comoros; Cook Islands; Djibouti; Egypt [64]; Ethiopia; Federated States of Micronesia; Fiji; French Polynesia [30, 42]; Guam; Hong Kong [64a]; India [58, 59]; Indonesia; Iran; Israel [64]; Jordan [64]; Japan [78a]; Kenya [43]; Kiribati; Kuwait; Madagascar [56, 57]; Malaysia [4]: including Sabah [101]; Maldives [20, 65]; Marshall Islands; Mauritius; Mozambique [8]; Myanmar; Nauru; New Caledonia [102]; Niue; Northem Marianas; Oman [66]; Palau; Papua New Guinea; Philippines [79]; Qatar; Reunion; Samoa; Saudi Arabia [1b]; Seychelles [65, 99]; Singapore; Solomon Islands; Somalia; Sri Lanka; Sudan [64]; Tanzania [43]; Taiwan [34]; Thailand [36, 72]; Tokelau; Tonga; Tuvalu; United Arab Emirates [9, 67]; Vanuatu [76]; Wallis and Futuna; Yemen

Hydnophora is a fairly common coral found in a range of reef habitats [100]. This genus has traditionally been included in the Family Faviidae, but has always been confused with Merulina, with which it has a close affinity. It has only recently been classified as a member of the Family Menulinidae [75]. Approximately 22 nominal species [75]; probably 6 valid species

Hydnophora bonsai Veron 1990

Hydnophora exesa (Pallas 1766)

Hydnophora grandis (Gardiner 1904)

Hydnophora laxa (Dana 1846)

Hydnophora microconos (Lamarck 1816)

Hydnophora pilosa Veron 1985

Hydnophora rigida (Dana 1846)

\section{Merulina Ehrenberg 1834}

(Red Sea [64], East and South Africa [8, 43]. Indian Ocean, north to Lacadives and Andaman and Nicobar Islands [59]; south to Madagascar and south-west Australia [80]. South-east Asia. Pacific Ocean, north to southern Japan, Northern Marianas and Marshall Islands; south to Great Barrier Reef, Lord Howe Island, Fiji and Samoa; east to Line Islands [75])

Countries listed without reference numbers are within the distribution range shown in Veron [75]

American Samoa [50]; Australia; British Indian Ocean Territory [65, 95]; Brunei; China [111, 112]; Christmas Island; Comoros; Djibouti; Egypt [64]; Ethiopia; Federated States of Micronesia; Fiji; Guam; Hong Kong [64a]; India [58, 59]; Indonesia; Israel [64]; Jordan [64]; Japan [78a]; Kenya [43]; Kiribati; Madagascar [56, 57]; Malaysia [4]: including Sabah [101]; Maldives [20, 65]; Marshall Islands; Mauritius; Myanmar; Nauru; New Caledonia [102]; Niue; Northern Marianas; Palau; Papua New Guinea; Philippines [79]; Reunion; Samoa; Saudi Arabia [1b]; Seychelles [65, 99]; Singapore; Solomon Islands; Somalia; Sri Lanka; Sudan [64]; Tanzania [43]; Taiwan [34]; Thailand [36, 72]; Tokelau; Tuvalu; Vanuatu [76]; Wallis and Futuna; Yemen

A relatively common coral, occurring in a wide range of reef habitats [100]. Collected for use as a curio. Six nominal species [75]; at least 3 valid species

Merulina ampliata (Ellis \& Solander 1786)

Merulina scabricula Dana 1846

Merulina scheeri Head 1983

\section{Paraclavarina Veron 1985}

(Australia (Great Barrier Reef) [82])

II

Indonesia [3]

Usually uncommon, although locally common in some reef lagoons with soft substrata [75]. The taxonomic status of this genus is still under debate. In 1991 [3] it was proposed that this species belonged in Merulina 
Scapophyllia Milne Edwards \& Haime 1848

11

(Andaman and Nicobar Islands [59]. Indonesia, south to Houtman Abrolhos (south-west Australia) [80]. South-east Asia. Pacific Ocean, north to Ryukyu Islands, Ogasawara-gunto lslands and Marshall Islands; south to Great Barrier Reef and New Caledonia; east to Fiji [75])

Countries listed without reference numbers are within the distribution range shown in Veron [75]

Australia; China [111, 112]; Federated States of Micronesia; Guam; India [58, 59]; Indonesia; Iran; Japan [78a]; Malaysia [4, 101]; Maldives [20, 65]; Marshall Islands; Myanmar; Nauru; New Caledonia [102]; Niue; Northern Marianas; Palau; Papua New Guinea; Philippines [79]; Singapore; Solomon Islands; Taiwan [34]; Thailand [36, 72]; Tuvalu; Vanuatu [76]

Uncommon, and usually found in slighily turbid water (e.g. lagoons) [75, 100].

1 species

Scapophyllia cylindrica Milne Edwards \& Haime 1848

Family FAVIIDAE Gregory 1900

Astreosmilia Ortmann 1892

11

British Indian Ocean Territory (Chagos Archipelago) [75]; East Africa [43]

One species, known from only a few specimens [75]

Astraeosmilia connata Ortmann 1892

Australogyra Veron \& Pichon 1982

II

Great Barrier Reef, Australia and Vanuatu [75]

Generally rare, and mostly restricted to turbid waters around high islands [75]. One species, originally described as Platygyra zelli [84]

Australogyra zelli (Veron, Pichon \& Wijsman-Best 1977)

Barabattoia Yabe \& Sugiyama 1941

II

(South-east Asia, south to south-western Australia and the Great Barrier Reef on the east [75]. Pacific Ocean, north to Ryukyu Islands and Marshall Islands; south to New Caledonia; east to Samoa and Tonga [75])

Countries listed without reference numbers are within the distribution range shown in Veron [75]

American Samoa [50]; Australia; Brunei; Christmas Island; Cocos (Keeling) lslands [77]; Federated States of Micronesia; Fiji; Hong Kong [64a]; Indonesia; Japan [78a]; Malaysia [4]: including Sabah [101]; Marshall Islands; Nauru; New Caledonia [102]; Niue; Palau; Papua New Guinea; Philippines [79]; Samoa; Singapore; Solomon Islands; Thailand [36, 72]; Tokelau; Tonga; Vanuatu [76]; Wallis and Futuna

An uncommon reef coral. Probably 4 nominal species and 3 valid species, although there is still some taxonomic confusion about this genus. Recent work $[75,84]$ suggests that Bikiniastrea is synonymous, although it is sometimes considered as a separate genus [100]

Barabattoia amicorum (Milne Edwards \& Haime 1850)

Barabattoia laddi (Wells 1954)

Barabattoia mirabilis Yabe \& Sugiyama 1941 
Caulastrea Dana 1846

11

(Red Sea [64], East and South Africa [8, 43]. Indian Ocean, north to Maldives [95] and Java; south to Madagascar and Dampier (north-west Australia). South-east Asia. Pacific, north to southern Japan, Ogasawara-gunto Islands and Marshall Islands; south to Great Barrier Reef, New Caledonia [102] and Tonga; east to Samoa [75])

Countries listed without reference numbers are within the distribution range shown in Veron [75]

Australia; Britisil Indian Ocean Territory [65, 95]; Brunei; China [111, 112]; Christmas Island; Federated States of Micronesia; Fiji; Guam; Indonesia; Japan [78a]; Kenya [43]; Madagascar [56, 57]; Malaysia [4]: including Sabah [101]; Marshall Islands; Mauritius; Mozambique [8]; Nauru; New Caledonia [102]; Niue; Northern Marianas; Palau; Papua New Guinea; Philippines [79]; Reunion; Samoa; Seychelles [65, 99]; Singapore; Solomon Islands; Tanzania [43]; Thailand [36, 72]; Tokelau; Tonga; Tuvalu; Vanuatu [76]; Wallis and Futuna

Eight nominal species, probably 4 valid species. Generally an uncommon coral, with the exception of $\mathrm{C}$. furcata, which is usually found on reef slopes $[69,100]$

Caulastrea curvata Wijsman-Best 1972

Caulastrea echinulata (Milne Edwards \& Haime 1849)

Caulastrea furcata Dana 1846

Caulastrea plana Hodgson \& Ross 1982

Caulastrea tumida Matthai 1928

Cladocora Ehrenberg 1834

(Caribbean [6I, 96], South Africa [8], Japan [103], Galapagos Islands [8])

Countries listed without reference numbers are within the distribution range shown in Smith [68], Veron [75] or Wood [100]

Anguilla; Antigua and Barbuda; Barbados; Belize [11]; Brazi] [49]; British Virgin Islands [37]; Cayman Islands; Colombia [62]; Costa Rica [33]; Cuba [48]; Dominica; Dominican Republic; Ecuador (Galapagos Islands) [8]; Grenada; Guadeloupe; Haiti; Honduras; Jamaica [96]; Martinique; Mexico [47]; Montserrat; Netherlands Antilles [47]; Nicaragua; Panama [61]; Puerto Rico; Saint Kitts and Nevis; Saint Lucia; Saint Vincent and the Grenadines; South Africa [8]; Trinidad and Tobago; Turks and Caicos; USA(Florida) [47]; Virgin Islands of the United States; Venezuela

There is a single shallow water species in the Caribbean, C. arbuscula [100]. This normally occurs on soft substrata in turbid water. Other species occur in deeper water. $0-274 \mathrm{~m}$.

4 valid species [25]

Cladocora arbuscula (Lesueur 1821)

Cladocora caespitosa (Linnaeus 1767)

Cladocora debilis Milne Edwards \& Haime 1849

Cladocora pacifica Cairns 1991

Cladorbicella Yabe \& Ehara 1936

II

1 species

Cladorbicella

Coelastrea Verrill 1866

I1

1 species

Coelastrea tenuis Verrill 1866 
Countries listed without reference numbers are within the distribution range shown in Smith [68] and Wood [100]

Anguilla; Antigua and Barbuda; Bahamas [47]; Barbados; Belize [11]; British Virgin Islands [37]; Cayman Islands; Colombia [62]; Costa Rica [33]; Cuba [48]; Dominica; Dominican Republic; Grenada; Guadeloupe; Haiti; Honduras; Jamaica [96]; Martinique; Mexico [47]; Montserrat; Netherlands Antilles [47]; Nicaragua; Panama [61]; Puerto Rico; Saint Kitts and Nevis; Saint Lucia; Saint Vincent and the Grenadines; Trinidad and Tobago; Turks and Caicos; USA(Florida) [47]; Virgin Islands of the United States; Venezuela

Widespread and important reef-builders [100]. Colpophyllia is sometimes included in the Family Trachyphylliidae.

3 species

Colpophyllia amaranthus (O.F. Müller 1775)

Colpophyllia breviserialis Milne Edwards \& Haime 1849

Colpophyllia natans (Houttuyn 1772)

\section{Cyphastrea Milne Edwards \& Haime 1848}

(Red Sea [64], Persian Gulf, East and South Africa [8, 43]. Indian Ocean, north to Arabian Gulf [9], Gulf of Kutch (north-eastern India) and Andaman and Nicobar Islands [59]; south to Madagascar, Cocos-Keeling Islands [87] and south-west tip of Australia [31]. South-east Asia. Pacific Ocean, north to southern Japan [96], Midway Islands and Hawaiian Islands [52], south to Lord Howe Island [75]; east to Tuamotu Archipelago [42])

Countries listed without reference numbers are within the distribution range shown in Veron [75]

American Samoa [50]; Australia; Bahrain [9, 16]; British lndian Ocean Territory [65, 95]; Brunei; China [111, 112]; Christmas Island; Cocos (Keeling) Islands [77]; Comoros; Cook Islands; Djibouti; Egypt [64]; Ethiopia; Federated States of Micronesia; Fiji; French Polynesia [30, 42]; Guam; Hawaiian Islands [52]; Hong Kong [64a]; India [58, 59]; Indonesia; Iran; Israel [64]; Jordan [64]; Japan [78a]; Kenya [43]; Kiribati: Kuwait; Madagascar [56, 57]; Malaysia [4]: including Sabah [101]; Maldives [20, 65]; Marshall Islands; Mauritius; Mozambique [8]; Myanmar; Nauru; New Caledonia [102]; Niue; Northern Marianas; Oman [66]; Palau; Papua New Guinea; Philippines [79]; Pitcairn Islands [54]; Qatar; Reunion; Samoa; Saudi Arabia [1b]; Seychelles [65, 99]; Singapore; Solomon Islands; Somalia; Sri Lanka; Sudan [64]; Tanzania [43]; Taiwan [34]; Thailand [36, 72]; Tokelau; Tonga; Tuvalu; United Arab Emirates [9, 67]; Vanuatu [76]; Wallis and Futuna; Yemen

Generally a fairly common coral, occurring in a range of reef habitats $[75,100]$. Twenty-six nominal species; possibly 8 valid species [75]

Cyphastrea agassizi (Vaughan 1907)

Cyphastrea chalcidicum (Forskảl 1775)

Cyphastrea decadia Moll \& Borel Best 1984

Cyphastrea gardineri Matthai 1914

Cyphastrea japonica Yabe \& Sugiyama 1932

Cyphastrea microphthalma (Lamarck 1816)

Cyphastrea ocellina (Dana 1846)

Cyphastrea serailia (Forskảl 1775)

Cyphastrea tanabensis Yabe \& Sugiyama 1932

Dendrocora Duncan 1876

Dendrocora fissipara Duncan 1876 
(Red Sea [64]. Aldabra and Madagascar [56] in the western Indian Ocean, north to Lacadives and Andaman and Nicobar Islands [59]; south to north-west Australia [31]. South-east Asia. Pacific Ocean, north to southern Japan, Northem Marianas, Marshall Islands and Phoenix Islands; south to Great Barrier Reef, New Caledonia and Fiji; east to Samoa $[75,100])$

Countries listed without reference numbers are within the distribution range shown in Veron [75]

American Samou [50]; Australia; British Indian Ocean Territory [65, 95]; Brunei; China [111, 112]; Christmas Island; Comoros; Djibouti; Egypt [64]; Ethiopia; Federated States of Micronesia; Fiji; Guam; Hong Kong [64a]; India [58, 59]; Indonesia; Japan [78a]; Madagascar [56, 57]; Malaysia [4]: including Sabah [101]; Maldives [20, 65]; Marshall Islands; Mauritius; Myanmar; Nauru; New Caledonia [102]; New Zealand; Niue; Northerm Marianas; Palau; Papua New Guinea; Philippines [79]; Reunion; Samoa; Saudi Arabia [1b]; Seychelles [65, 99]; Singapore; Solomon Islands; Sri Lanka; Sudan [64]; Taiwan [34]; Thailand [36, 72]; Tokelau; Tuvalu; Vanuatu [76]; Wallis and Futuna; Yemen

Relatively uncommon, although forms large colonies in a wide range of habitats [75, 41]. Collected for use as a building material. 1 species

Diploastrea heliopora (Lamarck 1816)

Diploria Milne Edwards \& Haime 1848

11

(Caribbean $[61,96]$. Bermuda $[25,41]$

Countries listed without reference numbers are within the distribution range shown in Smith [68] and Wood [100])

Anguilla; Antigua and Barbuda; Bahamas [47]; Barbados; Belize [11]; Bermuda [47]; British Virgin Islands [37]; Cayman 1slands; Colombia [62]; Costa Rica [33]; Cuba [48]; Dominica; Dominican Republic; Grenada; Guadeloupe; Haiti; Honduras; Jamaica [96]; Martinique; Mexico [47]; Montserrat; Netherlands Antilles [47]; Nicaragua; Panama [61]; Puerto Rico; Saint Kitts and Nevis; Saint Lucia; Saint Vincent and the Grenadines; Trinidad and Tobago; Turks and Caicos; USA(Florida) [47]; Virgin Islands of the United States; Venezuela

Diploria is an important reef-building coral, found in a wide range of habitats [68, 100]. Aproximately 12 nominal species; probably only 3 valid species

Diploria clivosa (Ellis \& Solander 1786)

Diploria labyrinthiformis (Linnaeus 1758)

Diploria strigosa (Dana 1848)

Echinopora Lamarck 1816

II

(Red Sea [64], East and South Africa [8, 43]. Indian Ocean, north to the Gulf of Mannar (southern India) and the Andaman and Nicobar Islands [59]; south to Madagascar [56], Cocos-Keeling Islands and Ningaloo Reefs (western Australia) [31]. South-east Asia. Pacific Ocean, north to southern Japan [71], Northern Marianas, Marshall Islands and Phoenix Islands; south to Great Barrier Reef [75]; east to Tuamotu Archipelago [42])

Countries listed without reference numbers are within the distribution range shown in Veron [75]

American Samoa [50]; Australia; British Indian Ocean Territory [65, 95]; Brunei; China [111, 112]; Christmas Island; Cocos (Keeling) 1slands [77]; Comoros; Cook 1slands; Djibouti; Egypt [64]; Ethiopia; Federated States of Micronesia; Fiji; French Polynesia [30, 42]; Guam; Hong Kong [64a]; India [58, 59]; Indonesia; Israel [64]; Jordan [64]; Japan [78a]; Kenya [43]; Madagascar [56, 57]; Malaysia [4]: including Sabah [101]; Maldives [20, 65]; Marshall 1slands; Mauritius; Mozambique [8]; Myanmar; Nauru; New Caledonia [102]; Niue; Northern Marianas; Oman [66]; Palau; Papua New Guinea; Philippines [79]; Pitcaim Islands [54]; Reunion; Samoa; Saudi Arabia [1b]; Seychelles [65, 99]; Singapore; Solomon 1slands; Somalia; Sri Larka; Sudan [64]; Tanzania [43]; Taiwan [34]; Thailand [36, 72]; Tokelau; Tonga; Tuvalu; Vanuatu [76]; Wallis and Futuna; Yemen

Generally a fairly common coral [100]. However, E. hirsutissima, although widely distributed, is very rare [75]. Collected for use as a curio. Approximately 30 nominal species, possibly 8 valid species. 
Echinopora ashmorensis Veron 1990

Echinopora forskaliana (Milne Edwards \& Haime 1850)

Echinopora gemmacea (Lamarck 1816)

Echinopora hirsutissima (Milne Edwards \& Haime 1849)

Echinopora horrida Dana 1846

Echinopora lamellosa (Esper 1795)

Echinopora mammiformis (Nemenzo 1959)

Echinopora pacificus Veron 1990

Echinopora rosularia Lamarck 1806

Echinopora spinulosa Brüggemann

Erythrastrea Scheer \& Pillai 1983

(Egypt (Gulf of Aqaba) [64])

Probably a single species, known from only a few specimens

Erythrastrea flabellata Scheer \& Pillai 1983

Erythrastrea wellsi (Ma 1959)

Favia Oken 1815

11

(Caribbean [61, 96] south to Brazil [49]. Bermuda [68, 100]. Eastern Atlantic, Cape Verde Islands, south to Ascension, east to Gulf of Guinea [30, 70]. Red Sea [64], Persian Gulf, East and South Africa [8, 43]. Indian Ocean, north to Arabian Gulf [9], Gulf of Kutch (north-west India), southern India, Andaman and Nicobar Islands [59] and Mergui Archipelago; south to Madagascar [56], Cocos-Keeling Islands [87] and south-west tip of Australia [31]. South-east Asia. Pacific Ocean, north to southern Japan [96] and Hawaiian Islands; south to Lord Howe Island and Pitcairn Islands [75]; east to Marquesas [42] and Easter Island [75])

Countries listed without reference numbers are within the distribution range shown in Smith [68], Veron [75] or Wood [100]

American Samoa [50]; Anguilla; Antigua and Barbuda; Australia; Bahamas [47]; Bahrain [9,16]; Barbados; Belize [I1]; Bermuda [47]; Brazil [49]; British Indian Ocean Territory [65, 95]; British Virgin Islands [37]; Brunei; Cape Verde [70]; Cayman Islands; China [111, 112]; Christmas Island; Cocos (Keeling) Jslands [77]; Colombia [62]; Comoros; Cook Islands; Costa Rica [33]; Cuba [48]; Djibouti; Dominica; Dominican Republic; Ecuador; Egypt [64]; Ethiopia; Federated States of Micronesia; Fiji; French Polynesia [30, 42]; Grenada; Guadeloupe; Guam; Haiti; Hawaiian Islands [52]; Honduras; Hong Kong [64a]; India [58, 59]; Indonesia; Iran; Israel [64]; Jamaica [96]; Japan [78a]; Jordan [64]; Kenya [43]; Kiribati; Kuwait; Madagascar [56, 57]; Malaysia [4]: including Sabah [101]; Maldives [20, 65]; Marshall Islands; Martinique; Mauritius; Mexico [47]; Montserrat; Mozambique [8]; Myanmar; Nauru; Netherlands Antilles [47]; New Caledonia [102]; New Zealand; Nicaragua; Niue; Northern Marianas; Oman [66]; Palau; Panama [61]; Papua New Guinea; Philippines [79]; Pitcairn Islands [54]; Puerto Rico; Qatar; Reunion; Saint Kitts and Nevis; Saint Lucia; Saint Vincent and the Grenadines; Samoa; Saudi Arabia [1b]; Seychelles [65, 99]; Singapore; Solomon Islands; Somalia; Sri Lanka; Sudan [64]; Tanzania [43]; Taiwan [34]; Thailand [36, 72]; Tokelau; Tonga; Trinidad and Tobago; Turks and Caicos; Tuvalu; United Arab Emirates [9, 67]; USA: California, Florida [52]; Vanuatu [76]; Virgin Islands of the United States; Venezuela; Wallis and Futuna; Yemen

Favia forms only small colonies in the Atlantic and is relatively uncommon [100]. In the Indo-Pacific it is an important reef-builder, occurring in all reef habitats. Some species are rare (e.g. $F$. helianthoides and $F$. maritima), but most are common and occur in a wide range of habitats. $F$. pallida is the most common and widespread species [75]. Collected for use as a curio, and also as a building material.

Approximately 70 nominal species; an unknown number of valid species; 19 are listed below. Eleven valid species are recorded from Australia [75], one from the Caribbean $(F$. fragum). F. leptophylla is endemic to Brazil [49]; $F$. gravida also occurs in the Western Brazil and Gulf of Guinea [49]

Favia affinis Milne Edwards \& Haime

Favia armata (Verrill 1872) 
Favia cavernosa (Forskål)

Favia danae Verrill 1872

Favia danai Milne Edwards \& Haime 1857

Favia deformis Eguchi \& Shirai

Favia elongata Eguchi \& Shirai

Favia favus (Forskảl 1775)

Favia fragum (Esper 1797)

Favia gravida Verrill 1868

Favia halicora Klunzinger

Favia helianthoides Wells 1954

Favia laxa (KJunzinger 1879)

Favia leptophylla Verrill 1868

Favia lizardensis Veron, Pichon \& Wijsman-Best 1977

Favia maritima (Nemenzo 1971)

Favia matthaii Vaughan 1918

Favia maxima Veron, Pichon \& Wijsman-Best 1977

Favia pallida (Dana 1846)

Favia rarisepta Eguchi \& Shirai

Favia rotulosa (Ellis \& Solander 1786)

Favia rotumana (Gardiner 1899)

Favia rotundata (Veron, Pichon \& Wijsman-Best 1977)

Favia speciosa (Dana 1846)

Favia stelligera (Dana 1846)

Favia valenciennesi (Milne Edwards \& Haime 1850)

Favia veroni Moll \& Borel Best 1984

Favia wisseli Scheer \& Pillai 1983

\section{Favites Link 1807}

II

(Red Sea [64], Persian Gulf, East and South Africa [8, 43]. Indian Ocean, north to Arabian Gulf [9] Lacadives, Gulf of Kutch (north-west India), southern India, Andaman and Nicobar Islands [59] and Mergui Archipelago; south to Madagascar [56] and Cocos-Keeling Islands [87] and south-west tip of Australia [31]. South-east Asia. Pacific Ocean, north to southern Japan [96], Ogasawara-gunto Islands, south to Lord Howe Island [75]; east to Tuamotu Archipelago [42])

Countries listed without reference numbers are within the distribution range shown in Veron [75]

American Samoa [50]; Australia; Bahrain [9. 67]; British Indian Ocean Territory [65, 95]; Brunei; China [111, 112]; Christmas Island; Comoros; Cook Islands; Djibouti; Egypt [64]; Ethiopia; Federated States of Micronesia; Fiji; French Polynesia [30, 42]; Guam; Hawaiian Islands [52]; Hong Kong [64a]; India [58, 59]; Indonesia; Iran; Israel [64]; Jordan [64]; Japan [78a]; Kenya [43]; Kiribati; Kuwait; Madagascar [56, 57]; Malaysia [4]: including Sabah [101]; Maldives [20, 65]; Marshall Islands; Mauritius; Mozambique [8]; Myanmar; Nauru; New Caledonia [102]; Niue; Northern Marianas; Palau; Papua New Guinea; Philippines [79]; Pitcairn Islands [54]; Qatar; Reunion; Samoa; Saudi Arabia [1b]; Seychelles [65, 99]; Singapore; Solomon Islands; Somalia; Sri Lanka; Sudan [64]; Tanzania [43]; Taiwan [34]; Thailand [36, 72]; Tokelau; Tonga; Tuvalu; United Arab Emirates [9, 67]; Vanuatu [76]; Wallis and Futuna; Yemen

Favites is an important reef-builder, but also occurs widely in non-reefal habitats. Most species are common and widespread $[75,100]$. Collected for use as a curio and also as a building material. Approximately 23 nominal species, an unknown number of valid species. Nine are listed below, seven of which are from Australia [75].

Favites abdita (Ellis \& Solander 1786)

Favites chinensis (Verrill 1866)

Favites complanata (Ehrenberg 1834)

Favites flexuosa (Dana 1846)

Favites halicora (Ehrenberg 1834)

Favites melicerum (Ehrenberg)

Favites palauensis (Yabe \& Sugiyama 1936) 
Favites pentagona (Esper 1794)

Favites peresi Faure \& Pichon 1978

Favites polarensis (Yabe \& Sugiyama 1936)

Favites rotundata (Veron, Pichon \& Wijsman-Best)

Favites rufa Wijsman-Best 1972

Favites russelli (Wells 1954)

Favites stylifera Yabe \& Sugiyama 1937

Goniastrea Milne Edwards \& Haime 1848

II

(Red Sea [64], Persian Gulf, East and South Africa [8, 43]. Indian Ocean north to Lacadives, Gulf of Kutch (north-west India) [59] and Mergui Archipelago; south to Madagascar and south-west tip of Australia. South-east Asia. Pacific Ocean, north to southern Japan [96] and Hawaiian Islands; south to Lord Howe 1sland [75]; east to Line Islands and Tuamotu Archipelago [42])

Countries listed without reference numbers are within the distribution range shown in Veron [75]

American Samoa [50]; Australia; Bahrain [9,67]; British Indian Ocean Territory [65, 95]; Brunei; China [111, 112]; Christmas Island; Comoros; Cook Islands; Djibouti; Egypt [64]; Ethiopia; Federated States of Micronesia; Fiji; French Polynesia [30, 42]; Guam; Hawaiian Islands [52]; Hong Kong [64a]; India [58, 59]; Indonesia; Iran; 1srael [64]; Jordan [64]; Japan [78a]; Kenya [43]; Kiribati; Kuwait; Madagascar [56, 57]; Malaysia [4]: including Sabah [101]; Maldives [20,65]; Marshall Islands; Mauritius; Mozambique [8]; Myanmar; Nauru; New Caledonia [102]; Niue; Northern Marianas; Palau; Papua New Guinea; Philippines [79]; Pitcaim Islands [54]; Qatar; Reunion; Samoa; Saudi Arabia [1b]; Seychelles [65, 99]; Singapore; Solomon lslands; Somalia; Sri Lanka; Sudan [64]; Tanzania [43]; Taiwan [34]; Thailand [36, 72]; Tokelau; Tonga; Tuvalu; United Arab Emirates [9, 67]; Vanuatu [76]; Wallis and Futuna; Yemen

An important reef-builder, but also occurs widely in non-reefal habitats. Most species are common and widespread [75, 100]. Collected for use as a curio and also as a building material. Approximately 34 nominal species; an unknown number of valid species. Eight listed below, seven of which are from Australia [75]

Goniastrea australensis (Milne Edwards \& Haime 1857)

Goniastrea deformis Veron 1990

Goniastrea edwardsi Chevalier 1971

Goniastrea favulus (Dana 1846)

Goniastrea palauensis (Yabe, Sugiyama \& Eguchi 1936)

Goniastrea pectinata (Ehrenberg 1834)

Goniastrea retiformis (Lamarck 1816)

Goniastrea varia (Dana)

Leptastrea Milne Edwards \& Haime 1848

I]

(Red Sea [64], Persian Gulf, East and South Africa [8, 43]. Indian Ocean, north to Arabian Gulf [9], Lacadives, Gulf of Kutch (north-west India) and Andaman and Nicobar Islands [59]; south to Madagascar, Cocos-Keeling Islands [87] and Houtman Abrolhos Islands (south-western Australia) [31]. South-east Asia. Pacific Ocean, north to southern Japan [96], Midway Islands and Hawaiian 1slands [52]; south to Elizabeth and Middleton Reefs (south-cast Australia) [31]; east to Line Islands, Tuamotu Archipelago [42] and Pitcaim Islands Island [75])

Countries listed without reference numbers are within the distribution range shown in Veron [75]

American Samoa [50]; Australia; Bahrain [9, 16]; British Indian Ocean Territory [65, 95]; Brunei; China [111, 112]; Christmas Island; Cocos (Keeling) Islands [77]; Comoros; Cook Islands; Djibouti; Egypt [64]; Ethiopia; Federated States of Micronesia; Fiji; French Polynesia [30, 42]; Guam; Hawaiian Islands [52]; Hong Kong [64a]; India [58, 59]; Indonesia; Iran; Israel [64]; Jordan [64]; Japan [78a]; Kenya [43]; Kiribati; Kuwait; Madagascar [56, 57]; Malaysia [4]: including Sabah [101]; Maldives [20, 65]; Marshall 1slands; Mauritius; Mozambique [8]; Myanmar; Nauru; New Caledonia [102]; Nive; Northern Marianas; Oman [66]; Palau; Papua New Guinea; Philippines [79]; Pitcairn Islands [54]; Qatar; Reunion; Samoa; Saudi Arabia [1b]; Seychelles [65, 99]; Singapore; Solomon Islands; Somalia; Sri Lanka; Sudan [64]; Tanzania [43]; Taiwan [34]; Thailand [36, 72]; Tokelau; Tonga; Tuvalu; United Arab Emirates [9, 67]; Vanuatu [76]; Wallis and Futuna; Yemen 
Most species, and particularly $L$. purpurea are common and occur in a wide range of reef habitats [75]. Sixteen nominal species; possibly 6 valid species, of which 5 are reported from Australia [75].

Leptastrea bewickensis Veron, Pichon \& Wijsman-Best 1977

Leptastrea botrae (Milne Edwards \& Haime 1849)

Leptastrea inaequalis Klunzinger 1879

Leptastrea pruinosa Crossland 1952

Leptastrea purpurea (Dana 1846)

Leptastrea transversa Klunzinger 1879

Leptoria Milne Edwards \& Haime 1848

II

(Red Sea [64], Persian Gulf, East and South Africa [8, 43]. Indian Ocean, north to Lacadives, Gulf of Mannar (southern India), Andaman and Nicobar Islands [59] and Mergui Archipelago; south to Madagascar, Cocos-Keeling Islands [87] and north-west Australia [31]. South-east Asia. Pacific Ocean, north to southern Japan, Ogasawara-gunto Islands and Phoenix Islands; south to Great Barrier Reef [75]; east to Tubauai Islands [42])

Countries listed without reference numbers are within the distribution range shown in Veron [75]

American Samoa [50]; Australia; Bahrain [9, 16]; British Indian Ocean Territory [65, 95]; Brunei; Christmas Island; Cocos (Keeling) Islands [77]; Comoros; Cook Islands; Djibouti; Egypt [64]; Ethiopia; Federated States of Micronesia; Fiji; French Polynesia [30, 42]; Guam; Hong Kong [64a]; India [58, 59]; Indonesia; Iran; Israel [64]; Jordan [64]; Japan [78a]; Kenya [43]; Kuwait; Madagascar [56, 57]; Malaysia [4]: including Sabah [101]; Maldives [20, 65]; Marshall Islands; Mauritius; Mozambique [8]; Myanmar; Nauru; New Caledonia [102]; New Zealand; Niue; Northern Marianas; Oman [66]; Palau; Papua New Guinea; Philippines [79]; Qatar; Reunion; Samoa; Saudi Arabia [ Ib]; Seychelles [65, 99]; Singapore; Solomon Islands; Somalia; Sri Lanka; Sudan [64]; Tanzania [43]; Taiwan [34]; Thailand [36, 72]; Tokelau; Tonga; Tuvalu; United Arab Emirates [9, 67]; Vanuatu [76]; Wallis and Futuna; Yemen

An important reef-building coral occurring in a wide range of habitats $[69,100]$. Collected for use as a building material.

2 species

Leptoria irregularis Veron 1990

Leptoria phrygia (Ellis \& Solander 1786)

Manicina Ehrenberg 1834

II

(Caribbean $[61,96])$

Countries listed without reference numbers are within the distribution range shown in Smith [68] and Wood [100]

Anguilla; Antigua and Barbuda; Bahamas [47]; Barbados; Belize [11]; Bermuda [47]; British Virgin Islands [37]; Cayman Islands; Colombia [62]; Costa Rica [33]; Cuba [48]; Dominica; Dominican Republic; Grenada; Guadeloupe; Haiti; Honduras; Jamaica [96]; Martinique; Mexico [47]; Montserrat; Netherlands Antilles [47]; Nicaragua; Panama [61]; Puerto Rico; Saint Kitts and Nevis; Saint Lucia; Saint Vincent and the Grenadines; Trinidad and Tobago; Turks and Caicos; USA(Florida) [47]; Virgin Islands of the United States; Venezuela

Manicina occurs attached, on reefs, or free-living in muddy or sandy areas [100]

I species

Manicina areolata (Linnaeus 1758)

Manicina mayori Wells 
Montastrea Blainville 1830

(Western Atlantic, Caribbean [61, 96] to Brazil [49]; Bermuda [68, 100]. Eastern Atlantic, Sao Thome and Principe [70]. Red Sea [64], Persian Gulf, East Africa and South Africa [8, 43]. Indian Ocean, north to Arabian Sea, southern India and Mergui Archipelago [75]; south to Madagascar and Houtman Abrolhos Islands (south-western Australia) [31]. South-east Asia. Pacific Ocean, north to southern Japan [71] and Line Islands; south to Lord Howe Island; east to Tuamotu Archipelago [30])

Countries listed without reference numbers are within the distribution range shown in Smith [68], Veron [75] or Wood [100]

American Samoa [50]; Anguilla; Antigua and Barbuda; Australia; Bahamas [47]; Bahrain [9,16]; Barbados; Belize [11]; Bermuda [47]; Brazil [49]; British Indian Ocean Territory [65, 95]; British Virgin Islands [37]; Brunei; Cayman Islands; Christmas Island; Cocos (Keeling) Islands [77]; Colombia [62]; Comoros; Cook Islands; Costa Rica [33]; Cuba [48]; Djibouti; Dominica; Dominican Republic; Egypt [64]; Ethiopia; Federated States of Micronesia; Fiji; French Polynesia [30, 42]; Grenada; Guadeloupe; Guam; Haiti; Honduras; Hong Kong [64a]; India [58, 59]; Indonesia; Iran; Israel [64]; Jamaica [96]; Japan [78a]; Jordan [64]; Kenya [43]; Kiribati; Kuwait; Madagascar [56, 57]; Malaysia [4]: including Sabah [101]; Maidives [20, 65]; Marshall Islands; Martinique; Mauritius; Mexico [47]; Montserrat; Mozambique [8]; Myanmar; Nauru; Netherlands Antilles [47]; New Caledonia [102]; Nicaragua; Niue; Northern Marianas; Palau; Panama [61]; Papua New Guinea; Philippines [79]; Pitcairn Islands [54]; Puerto Rico; Qatar; Reunion; Saint Kitts and Nevis; Saint Lucia; Saint Vincent and the Grenadines; Samoa; Saudi Arabia [1b]; Seychelles [65, 99]; Singapore; Solomon Islands; Somalia; Sri Lanka; Sudan [64]; Tanzania [43]; Taiwan [34]; Thailand [36, 72]; Tokelau; Tonga; Trinidad and Tobago; Turks and Caicos; Tuvalu; United Arab Emirates [9, 67]; United States: Florida; Vanuatu [76]; Virgin Islands of the United States; Venezuela; Wallis and Futuna; Yemen

In the western Atlantic Montastrea is a common and important reef-buiding coral [96, 100]. In the Indo-Pacific; only $M$. curta is common, the other species are generally uncommon, although occur in a range of reef habitats $[75,100]$. Collected for use as a building material in the lndo-Pacific.

Approximately 15 nominal species. Two valid species in the Western Atlantic, at least 5 in the lndo-Pacific

Montastrea annularis (Ellis \& Solander 1786)

Montastrea annuligera (Milne Edwards \& Haime 1849)

Montastrea aperta (Verrill)

Montastrea cavernosa (Linnaeus 1767/6?)

Montastrea curta (Dana 1846)

Montastrea magnistellata Chevalier 1971

Montastrea multipunctata Hodgson 1985

Montastrea valenciennesi (Milne Edwards \& Haime 1848)

Moseleya Quelch 1884

II

(Philippines, south to Houtman Abrolhos Islands (south-western Australia) and Great Barrier Reef (eastern Australia) [75])

Usually uncommon and restricted to turbid, shallow water [75]

1 species

Moseleya latistellata Quelch 1884

Oulastrea Milne Edwards \& Haime 1848

li

(South-east Asia [100], south to northern Australia [31]. Western Pacific Ocean, north to southern Japan, south to New Guinea and the Solomon Islands [75])

Countries listed without reference numbers are within the distribution range shown in Veron [75]

Australia; Brunei; Federated States of Micronesia; Guam; Hong Kong [64a]; India [58, 59]; Indonesia; Japan [78a]; Malaysia [4, 101]; Myanmar; Palau; Papua New Guinea; Philippines [79]; Singapore; Solomon Islands; 
Thailand [36, 72]; Wallis and Futuna; Yemen

This species is generally restricted to shallow, often muddy water, in back reef areas $[75,100]$. Sometimes included in the Siderastreidae [32].

3 nominal species; 1 valid species

Oulastrea crispata (Lamarck 1816)

Oulophyllia Milne Edwards \& Haime 1848

II

(Red Sea [64], Persian Gulf, East and South Africa [8, 43]. Indian Ocean, north to Andaman and Nicobar Islands [59] and Mergui Archipelago; south to Madagascar and Ningaloo Reefs (north-west Australia) [31]. South-east Asia. Pacific Ocean, north to southern Japan [71] and Marshall islands; south to Great Barrier Reef and Fiji; east to Phoenix Islands and Samoa [75])

Countries listed without reference numbers are within the distribution range shown in Veron [75]

American Samoa [50]; Australia; Bahrain [9, 16]; British Indian Ocean Territory [65, 95]; Brunei; Christmas Island; Comoros; Djibouti; Egypt [64]; Ethiopia; Federated States of Micronesia; Fiji; French Polynesia [30, 42]; Guam; India [58, 59]; Indonesia; Japan [78a]; Kenya [43]; Kiribati; Kuwait; Madagascar [56, 57]; Malaysia [4]: including Sabah [101]; Maldives [20, 65]; Marshall Islands; Mauritius; Mozambique [8]; Myanmar; Nauru; New Caledonia [102]; Niue; Northern Marianas; Palau; Papua New Guinea; Philippines [79]; Qatar; Reunion; Samoa; Saudi Arabia [1b]; Seychelles [65, 99]; Singapore; Solomon Islands; Somalia; Sri Lanka; Sudan [64]; Tanzania [43]; Taiwan [34]; Thailand [36, 72]; Tokelau; Tuvalu; United Arab Emirates [9, 67]; Vanuatu [76]; Wallis and Futuna; Yemen

Occurs in most reef habitats, but generally uncommon. $O$. crispa is the most widely distributed species $[75,100]$ Collected for use as a building material. Approximately 11 nominal species, at least 2 valid species [75].

Oulophyllia bennettae (Veron, Pichon \& Wijsman-Best 1977)

Oulophyllia crispa (Lamarck 1816)

Parasimplastrea Sheppard 1985

Oman [66]

1 species

Parasimplastrea simplicitexta (Umbgrove 1939)

Platygyra Ehrenberg 1834

II

(Red Sea [64], Persian Gulf, East and South Africa [8, 43]. Indian Ocean, north to Arabian Gulf [9], Lacadives, Gulf of Kutch (north-west India), southern India, Andaman and Nicobar Islands [59] and Mergui Archipelago; south to Madagascar and south-western Australia [31]. South-east Asia. Pacific Ocean, north to southern Japan [96] and Line Islands; south to Lord Howe Jsland and Kermadec Islands [75]; east to Tuamotu Archipelago [42])

Countries listed without reference numbers are within the distribution range shown in Veron [75]

American Samoa [50]; Australia; Bahrain [9, 16]; British Indian Ocean Territory [65, 95]; Brunei; China [111, 112]; Christmas lsland; Comoros; Cook Islands; Djibouti; Egypt [64]; Ethiopia; Federated States of Micronesia; Fiji; French Polynesia [30, 42]; Guam; Hong Kong [64a]; India [58, 59]; Indonesia; Iran; Israel [64]; Jordan [64]; Japan [78a]; Kenya [43]; Kiribati; Kuwait; Madagascar [56, 57]; Malaysia [4]: including Sabah [101]; Maldives [20, 65]; Marshall lslands; Mauritius; Mozambique [8]; Myanmar; Nauru; New Caledonia [102]; New Zealand: Kermadec Islands; Niue; Northern Marianas; Oman [66]; Palau; Papuz New Guinea; Philippines [79]; Qatar; Reunion; Samoa; Saudi Arabia [1b]; Seychelles [65, 99]; Singapore; Solomon Islands; Somalia; Sri Lanka; Sudan [64]; Tanzania [43]; Taiwan [34]; Thailand [36, 72]; Tokelau; Tonga; Tuvalu; United Arab Emirates [9, 67]; Vanuatu [76]; Wallis and Futuna; Yemen 
$P$. daedalea is the commonest species, the others are generally less common, although occur in a wide range of reef habitats $[75,100]$. Collected for use as a curio, and as a building material. Approximately 26 nominal species; an unknown number of valid species. Nine listed here, of which five are recognised from Australia [75]

Platygyra contorta Veron 1990

Platygyra crosslandi (Matthai 1928)

Platygyra daedalea (Ellis \& Solander 1786)

Platygyra lamellina Ehrenberg 1834

Plarygyra pini Chevalier 1975

Platygyra ryukyuensis Yabe \& Sugiyama 1936

Platygyra sinensis (Milne Edwards \& Haime 1849)

Platygyra verweyi Wijsman-Best 1976

Platygyra yaeyamaensis (Eguchi \& Shirai 1977)

Plesiastrea Milne Edwards \& Haime 1848

II

(Red Sea [64], Persian Gulf, East and South Africa [8, 43]. Indian Ocean, north to Arabian Gulf [9], Gulf of Kutch and Andaman and Nicobar Islands [59]; south to Madagascar and entire south coast of Australia [75]. South-east Asia. Pacific Ocean, north to southern Japan [96] and Line Islands; south to south-east tip of Australia and Lord Howe Island [75]; east to Tuamotu Archipelago [42] and Pitcairn Islands Island)

Countries listed without reference numbers are within the distribution range shown in Veron [75]

American Samoa [50]; Australia; Bahrain [9, 16]; British lndian Ocean Territory [65, 95]; Brunei; China [111, 112]; Christmas Island; Cocos (Keeling) Islands [77]; Comoros; Cook Islands; Djibouti; Egypt [64]; Ethiopia; Federated States of Micronesia; Fiji; French Polynesia [30, 42]; Guam; Hong Kong [64a]; India [58, 59]; Indonesia; Iran; Israel [64]; Jordan [64]; Japan [78a]; Kenya [43]; Kiribati; Kuwait; Madagascar [56, 57]; Malaysia [4]: including Sabah [101]; Maldives [20, 65]; Marshall Islands; Mauritius; Mozambique [8]; Myanmar; Nauru; New Caledonia [102]; Niue; Northern Marianas; Oman [66]; Palau; Papua New Guinea; Philippines [79]; Pitcairn Islands [54]; Qatar; Reunion; Samoa; Saudi Arabia [1b]; Seychelles [65, 99]; Singapore; Solomon Islands; Somalia; Sri Lanka; Sudan [64]; Tanzania [43]; Taiwan [34]; Thailand [36, 72]; Tokelau; Tonga; Tuvalu; United Arab Emirates [9, 67]; Vanuatu [76]; Wallis and Futuna; Yemen

Widely distributed, occurring in a wide range of non-reefal habitats where it may form large colonies. It is less conspicuous on tropical reefs, but occurs in most habitats [101]. Nine nomital species; possibly one valid species

Plesiastrea lilli Wells 1954

Plesiastrea versipora (Lamarck 1816)

Solenastrea Milne Edwards \& Haime 1848

(Caribbean $[61,96])$

II

Countries listed without reference numbers are within the distribution range shown in Smith [68] and Wood [100]

Anguilla; Antigua and Barbuda; Barbados; Brazil [49]; British Virgin Islands [37]; Cayman Islands; Colombia [62]; Costa Rica [33]; Dominica; Dominican Republic; Grenada; Guadeloupe; Haiti; Honduras; Jamaica [96]; Martinique; Mexico [47]; Montserrat; Nicaragua; Panama [61]; Puerto Rico; Saint Kitts and Nevis; Saint Lucia; Saint Vincent and the Grenadines; Trinidad and Tobago; Turks and Caicos; USA(Florida) [47]; Virgin Islands of the United States; Venezuela

This coral forms small colonies and tends to occur on deeper reefs [100]

2 species

Solenastrea bournoni Milne Edwards \& Haime 1850

Solenastrea hyades (Dana 1846) 
RL Ref

Family TRACHYPHYLL11DAE Verrill 1901

Callogyra Verrill 1902

1 species

\section{Callogyra formosa Verrill 1902}

\section{Trachyphyllia Audouin 1826}

(Red Sea [64], East Africa [8]. Indian Ocean, north to Maldives and Andaman and Nicobar Islands [59]; south to Madagascar and Dampier (north-west Australia) [31]. South-east Asia. Pacific Ocean, north to southern Japan, south to Great Barrier Reef and New Caledonia [75])

Countries listed without reference numbers are within the distribution range shown in Veron [75]

Australia; British Indian Ocean Territory [65, 95]; Christmas Island; Comoros; Djibouti; Egypt [64]; Ethiopia; India [58, 59]; Indonesia; Israel [64]; Jordan [64]; Japan [78a]; Kenya [43]; Madagascar [56, 57]; Malaysia [4, 101]; Maldives [20, 65]; Mauritius; Mozambique [8]; New Caledonia [102]; Papua New Guinea; Philippines [79]; Reunion; Saudi Arabia [1b]; Seychelles [65, 99]; Singapore; Solomon Islands; Sudan [64]; Tanzania [43]; Thailand [36, 72]; Yemen

A free-living coral, rare on reefs, but may be common in shallow, sandy areas between coral outcrops [100]. Collected for use live in aquaria, and as a curio. Probably 6 nominal species; possibly only 1 species.

Trachyphyllia geoffroyi (Audouin 1826)

Wellsophyllia Pichon 1980 II

(Philippines; Borneo and 1ndonesia south to the coast of northern Australia [75])

There is little information on the distribution and ecology of this species, which is possibly an ecomorph of Trachyphyllia geoffroyi

1 species

Wellsophyllia radiata Pichon 1980

Family MEANDRINIIDAE Gray 1847

Ctenella Matthai 1928

II

(Chagos Archipelago [23])

1 species

\section{Crenella chagius Matthai 1928}

Dendrogyra Ehrenberg 1834

(Caribbean [40])

Countries listed without reference numbers are within the distribution shown in Smith [68] and Wood [100]

Anguilla; Antigua and Barbuda; Bahamas [47]; Barbados; Belize [11]; British Virgin Islands [37]; Cayman Islands; Costa Rica [33]; Cuba [48]; Dominica; Dominican Republic; Grenada; Guadeloupe; Haiti; Honduras; Jamaica [96]; Martinique; Montserrat; Netherlands Antilles [47]; Nicaragua; Puerto Rico; Saint Kitts and Nevis; Saint Lucia; Saint Vincent and the Grenadines; Trinidad and Tobago; Turks and Caicos; USA(Florida) [47]; Virgin Islands of the United States; Venezuela

A relatively uncommon coral, but forms large colonies [100]. Collected for use as a curio. 1 species 
Dichocoenia Milne Edwards \& Haime 1848

II (Caribbean [61, 96], Bermuda [68, 100])

Countries listed without reference numbers are within the distribution range shown in Smith [68] and Wood [100]

Anguilla; Antigua and Barbuda; Bahamas [47]; Barbados; Belize [11]; Bermuda [47]; British Virgin Islands [37]; Cayman Islands; Colombia [62]; Costa Rica [33]; Cuba [48]; Dominica; Dominican Republic; Grenada; Guadeloupe; Haiti; Honduras; Jamaica [96]; Martinique; Mexico [47]; Montserrat; Netherlands Antilles [47]; Nicaragua; Panama [61]; Puerto Rico; Saint Kitts and Nevis; Saint Lucia; Saint Vincent and the Grenadines; Trinidad and Tobago; Turks and Caicos; USA(Florida) [47]; Virgin Islands of the United States; Venezuela

Fairly common, especially on deeper reefs [100]. 2 species

Dichocoenia stellaris Milne Edwards \& Haime 1849

Dichocoenia stokesi Milne Edwards \& Haime 1848

Meandrina Lamarck 1801

(Western Atlantic, Caribbean $[61,96]$ to Brazil [49]; Bermuda $[68,100]$ )

Countries listed without reference numbers are within the distribution range shown in Smith [68] and Wood [100]

Anguilla; Antigua and Barbuda; Bahamas [47]; Barbados; Belize [11]; Bermuda [47]; Brazil [49]; British Virgin Islands [37]; Cayman Islands; Colombia [62]; Costa Rica [33]; Cuba [48]; Dominica; Dominican Republic; Grenada; Guadeloupe; Haiti; Honduras; Jamaica [96]; Martinique; Mexico [47]; Montserrat; Netherlands Antilles [47]; Nicaragua; Panama [61]; Puerto Rico; Saint Kitts and Nevis; Saint Lucia; Saint Vincent and the Grenadines; Trinidad and Tobago; Turks and Caicos; USA(Florida) [47]; Virgin Islands of the United States; Venezuela

This and some specimens of the Caribbean species are unattached and live on soft substrata around reefs. Attached colonies are larger and occur in a variety of habitats [100]

At least 2 species

Meandrina alveolus (Duncan 1863)

Meandrina meandrites (Linnaeus 1758)

Meandrina memorialis (Wells 1974)

Meandrina spinulosa (Dana 1846)

Family ANTHEMIPHYLLIIDAE Vaughan 1907

Anthemiphyllia Pourtalès 1878

West Indies, Japan [40], Pacific Ocean [75]; Japan \& Hawaii [10]

Solitary; free living. Deep water, Approximately 500-700m [10]. Possibly 3 species [10].

Anthemiphyllia dentata (Alcock 1902)

Anthemiphyllia pacifica Vaughan 1907

Anthemiphyllia patera Pourtalès 1878 


\section{Family CARYOPHYLLIIDAE Gray 1847}

The Family is sometimes separated into the Families Caryophylliidae, Desmophyllidae, Parasmilidae, Turbinoliidae and Eusmilidae [32], but maintained in subfamilies by other (recent) authorities [e.g. 25, 75, 107]. The majority of genera in this Family are non-reefal, ahermatypic, solitary corals, often from deep water. They may be attached or free-living. In most cases their distribution and the status of populations is poorly known (relying on dredge samples)

Anomocora Studer, 1878

II

(Western Atlantic; Red Sea; Sumatra; Japan; Hawaii; Galapagos Islands. 130-540m [25])

Two described species; possibly others [25]

Anomocora carinata Caims 1991

Anomocora fecunda (Pourtalès 1871)

Asterosmilia Duncan 1868

11

(Atlantic [3, 46]. Indian Ocean, South Africa, Japan [107]. Approximately32-311m depth)

An unknown number of species

Asterosmilia marchadi (Chevalier 1966)

Asterosmilia prolifera (Pourtalès 1871)

Aulocyathus von Marenzeller 1904

II

(Worldwide, including Antarctica $[12,75,107]$. Approximately $450-1,300 \mathrm{~m}$ depth)

One species has been recorded from the North-East Atlantic [107]; one species from Australia [107]

Aulocyathus atlanticus Zibrowius 1980

Aulocyathus conotrochoides (Yabe \& Eguchi 1932)

Aulocyathus juvenescens von Marenzeller 1904

Aulocyathus recidivus Dennant 1906

Australocyathus Cairns \& Parker 1992?

1 species

Australocyathus vincentinus (Dennant 1906)

Bourneotrochus Wells 1984

11

(Vanuatu, Hawaii and Australia [75]. Deep to very deep water)

One species recorded from Australia [16]

Bourneotrochus stell(ul)atus (Cairns 1984)

Caryophyllia Lamarck 1801

(Cosmopolitan, including Antarctica $[28,45] .0-3200 \mathrm{~m}$ )

57 recent species $[28,109]$

Caryophyllia abyssorum Duncan 1873

Caryophyllia alaskana Vaughan 1941 ?alaskensis

Caryophyllia alberti Zibrowius 1980

Caryophyllia ambrosia Alcock 1898

Caryophyllia antarctica Marenzeller 1904

Caryophyllia antillarum Pourtalès 1874 
Caryophyllia arcuata Milne Edwards \& Haime Caryophyllia arnoldi Vaughan 1900

Caryophyllia atlantica (Duncan 1873)

Caryophyllia balaenacea Zibrowius \& Gili 1990

Caryophyllia barbadensis Caims 1979

Caryophyllia berteriana Duchassaing 1850

Caryophyllia calveri Duncan 1873

Caryophyllia capensis Gardiner 1904

Caryophyllia clavus Wells 1958

Caryophyllia cornuformis Pourtalès 1868

Caryophyllia corrugata Caims 1979

Caryophyllia cultrifera Alcock 1902

Caryophyllia cyathus (Ellis \& Solander 1786)

Caryophyllia diomedeae Marenzeller 1904

Caryophyllia eltaninae Cairns 1982

Caryophyllia ephyala Alcock 1891

Caryophyllia epithecata sensu Gardiner 1904

Caryophyllia foresti Zibrowius 1980

Caryophyllia grandis Gardiner \& Waugh 1938

Caryophyllia grayi (Milne Edwards \& Haime 1848)

Caryophyllia hawaiiensis Vaughan 1907

Caryophyllia horologium Cairns 1977

Caryophyllia inornata (Duncan 1878)

Caryophyllia japonica Marenzeller 1888

Caryophyllia jogashimaensis Eguchi 1968

Caryophyllia lamellifera Moseley 1881

Caryophyllia mabahithi Gardiner \& Waugh 1938

Caryophyllia marmorea Cairns 1984

Caryophyllia oczopali Vaughan 1907

Caryophyllia panda Alcock 1902

Caryophyllia paradoxus Alcock 1898

Caryophyllia parvula Cairns 1979

Caryophyllia paucipalata Moseley 1881

Caryophyllia pauciseptata Yabe \& Eguchi 1932

Caryophyllia perculta Cairns 1991

Caryophyllia planilamellata Dennant 1906

Caryophyllia polygona Pourtalès 1878

Caryophyllia profunda Moseley 1881

Caryophyllia quadragenaria Alcock 1902

Caryophyllia rugosa Moseley 1881

Caryophyllia sarsiae Zibrowius 1974

Caryophyllia scillaemorpha Alcock 1894

Caryophyllia scobinosa Alcock 1902

Caryophyllia seguenzae Duncan 1873

Caryophyllia sewelli Gardiner \& Waugh 1938

Caryophyllia smithii Stokes \& Broderip 1828

Caryophyllia solida Caims 1991

Caryophyllia squiresi Caims 1982

Caryophyllia transversalis Moseley 1881

Caryophyllia valdiviae Zibrowius \& Gili 1990

Caryophyllia zopyros Caims 1979

Catalaphyllia Wells 1971 ?Catalophyllia

(Aldabra and Seychelles, east to Dampier (north-west Australia). South-east Asia. Pacific Ocean, north to Japan [40], east to Solomon Islands and Vanuatu; south to Great Barrier Reef and New Caledonia [75])

Australia; Christmas Island; Federated States of Micronesia; Indonesia; Japan [78a]; Malaysia [4, 101]; Maldives [65]; Myanmar; New Caledonia [102]; Palau; Papua New Guinea; Philippines [79]; Samoa; Seychelles [65]; 
Singapore; Solomon Islands; Thailand [36, 72]

Uncommon and occurs only in turbid water habitats. Collected for use live in aquaria.

1 species

Catalaphyllia jardinei (Saville-Kent 1893)

Catalaphyllia okinawensis Eguchi \& Shirai 1977

Catalaphyllia sabiuraensis Eguchi

Ceratotrochus Milne Edwards \& Haime 1848

II

(Mediterranean [107], South Africa [8], Japan [44]; USA: Gulf of California [44]; Hawaiian Islands [16]. Approximately $7-400 \mathrm{~m}$ depth)

Possibly only one or two species [107]

Ceratotrochus laxus Vaughan 1907

Ceratotrochus magnaghii Cecchini 1914

Coenocyathus Milne Edwards \& Haime 1848 II

(Mediterranean, North-east Atlantic [107]. Approximately $100-300 \mathrm{~m}$ depth)

Possibly 6 species; two are reported from the North-east Atlantic [107]

Coenocyathus anthophyllites Milne Edwards \& Haime 1848

Coenocyathus bowersi Vaughan 1906

Coenocyathus cylindricus Milne Edwards \& Haime 1848

Coenosmilia Pourtalès 1874

(Pacific Ocean. Approximately 109-622m depth)

One species recorded from Hawaii

Coenosmilia inordinata Cairns 1984

Concentrotheca Cairns 1979

(Western Atlantic; Azores; Galapagos Islands. 183-800m [10, 28])

2 species

Concentrotheca laevigata (Pourtalès 1871)

Concentrotheca vaughani Cairns 1991

Conocyathus D'Orbigny 1851

(Indo-Pacific [75]. Shallow water)

II

One species recorded from Australia [75]

Conocyathus zelandiae Duncan 1876

Conotrochus Seguenza 1864/3?

(Indo-Pacific to Hawaii [52]. Deep water)

Conotrochus brunneus Moseley 1881

Conotrochus funicolumna (Alcock 1902) 
3 species

Crispatotrochus galapagensis Cairns 1991

Crispatotrochus niinoi (Yabe \& Eguchi 1942)

Crispatotrochus tydmani (Alcock 1902)

Cryptotrochus Caims 1988

1 species

Cryptotrochus carolinensis Caims 1988

Cyathoceras Moseley 1881 = ?Crispatotrochus

II

(Indo-Pacific [75] including Hawaii [16]; Western Atlantic [10]. 220-1,097m depth)

At least 7 species

Cyathoceras cornu Moseley 1881

Cyathoceras foxi Durham \& Barnard 1952

Cyathoceras inornatus (Tenison-Woods 1878)

Cyathoceras irregularis Cairns 1982

Cyathoceras rubescens Moseley 1881

Cyathoceras squiresi Cairns 1979

Cyathoceras woodsi Wells 1964

Cylindrophyllia Yabe \& Eguchi 1937 (Pacific Ocean [75])

One species has been recorded from Australia, probably undescribed [75]. Deep water

Dactylotrochus Wells 1954

(Red Sea, Indo-Pacific [64]. Deep water)

II

Unknown number of species

Dactylotrochus cervicornis (Moseley 1881)

Dasmosmilia Pourtalès 1880

II

(Atlantic Ocean; West Africa; Red Sea; possibly Pacific Ocean [107]. Approximately 70-500m depth)

Unknown number of species; two are reported from the Red Sea and two from the North-east Atlantic [107]. Parasmilia is probably synonymous [107]

Dasmosmilia lymani (Pourtalès 1871)

Dasmosmilia valida Marenzeller 1907

Dasmosmilia variegata (Pourtalès 1871)

Delocyathus Milne Edwards \& Haime 1848

II

(Cosmopolitan, including Antarctica [10,45]. Approximately $80-2300 \mathrm{~m}$ depth)

Six species recorded from Curacao [10]; three from North-East Atlantic [107]; three from Australia [75]. 12 species listed here, possibly more are valid

Deltocyathus agassizii Pourtalès 1867

Deltocyathus andamanicus Alcock 1892 
Deltocyathus calcar Pourtalès 1874

Delrocyathus conicus Zibrowius 1980

Deltocyathus eccentricus Caims 1979

Deltocyathus italicus (Michelotti 1838)

Deltocyathus japonicus (Yabe \& Eguchi 1932)

Deltocyathus minutus Gardiner \& Waugh 1938

Delocyathus moseleyi Caims 1979

?Deltocyathus ornatus Gardiner 1899

Delrocyathus pournalesi Caims 1979

Deltocyathus stellulatus Caims 1984

Deltocyathus vincentinus Dennant 1904

Desmophyllum Ehrenberg 1834

(Widely distributed in Atlantic, Pacific and Indian Oceans, except off continental Antarctica [25]. Approximately $35-2,600 \mathrm{~m}$ depth)

3 valid species listed, probably others occur

Desmophyllum cristagalli Milne Edwards \& Haime 1848

Desmophyllum reesei Duchassaing \& Michelotti 1864

Desmophyllum striatum Cairns 1979

Desmophyllum tenuescens Gardiner 1899

Dunocyathus Tenison-Woods 1878

II

(Known only from Australia (southern Queensland to Tasmania) [75]. Deep water)

One species has been recorded

Dunocyathus parasiticus Tenison-Woods 1878

Edwardsotrochus Chevalier 1961

4 species

Endocyathopora Cairns 1989

Philippines. 46-70m [23]

1 species

Endocyathopora laticostata Cairns 1989

\section{Euphyllia Dana 1846}

II

(Red Sea [64], East Africa [43]. Indian Ocean, north to Lacadives and Andaman Islands [59]; south to Madagascar and Houtman Abrolhos Islands (south-west Australia) [80]. South-east Asia. Pacific Ocean, north to southern Japan [40] and Marshall Islands; south to Lord Howe Island and Fiji; east to Samoa [75])

Countries listed without reference numbers are within the distribution range shown in Veron [75]

American Samoa [50]; Australia; British Indian Ocean Territory [65, 95]; Brunei; China [111, 112]; Christmas Island; Comoros; Djibouti; Egypt [64]; Ethiopia; Federated States of Micronesia; Fiji; Guam; Hong Kong [64a]; India [58, 59]; Indonesia; Japan [78a]; Kenya [43]; Madagascar [56, 57]; Malaysia [4]: including Sabah [10I]; Maldives [20, 65]; Marshall Islands; Mauritius; Mozambique [8]; Myanmar; Nauru; New Caledonia [102]; Niue; Northern Marianas; Oman [66]; Palau; Papua New Guinea; Philippines [79]; Reunion; Samoa; Saudi Arabia [1b]; Seychelles [65, 99]; Singapore; Solomon Islands; Somalia; Sri Larka; Sudan [64]; Tanzania [43]; Taiwan [34]; Thailand [36, 72]; Tokelau; Tuvalu; Vanuatu [76]; Wallis and Futuna

Euphyllia is found in a wide range of reef habitats, and is a relatively common coral [41]

Collected for use live in aquaria and for use as a curio. At least 8 valid species $[75,78]$ 
Euphyllia ancora Veron \& Pichon 1980

Euphyllia cristata Chevalier 1971

Euphyllia divisa Veron \& Pichon 1980

Euphyllia fimbriata (Spengler 1799)

Euphyllia glabrescens (Chamisso \& Eysenhardt 1821)

Euphyllia kabiraensis?

Euphyllia paraancora Veron 1990

Euphyllia paradivisa Veron 1990

Euphyllia paraglabrescens Veron 1990

Euphyllia picteti Bedol

Euphyllia yaeyamaensis (Shirai 1980)

Eusmilia Milne Edwards \& Haime I848

II

(Caribbean [96])

Countries listed without reference numbers are within the distribution range shown in Smith [68] and Wood [100]

Anguilla; Antigua and Barbuda; Bahamas [47]; Barbados; Belize [11]; Brazil [49]; British Virgin Islands [37]; Cayman Islands; Colombia [62]; Costa Rica [33]; Cuba [48]; Dominica; Dominican Republic; Grenada; Guadeloupe; Haiti; Honduras; Jamaica [96]; Martinique; Mexico [47]; Montserrat; Netherlands Antilles [47]; Nicaragua; Panama [61]; Puerto Rico; Saint Kitts and Nevis; Saint Lucia; Saint Vincent and the Grenadines; Trinidad and Tobago; Turks and Caicos; USA(Florida) [47]; Virgin Islands of the United States; Venezuela

I species

Eusmilia fastigiata (Pallas 1766)

Goniocorella Yabe \& Eguchi 1932

11

(Off Japan; Banda Sea, off New Zealand [12])

Goniocorella dumosa (Alcock 1902)

Gyrosmilia Milne Edwards \& Haime 1851

II

Djibouti; Egypt; Ethiopia; Israel; Kenya; Mauritius; Japan [78a]; Saudi Arabia; Seychelles: Aldabra; Somalia; Sudan; Tanzania; Reunion; Yemen [65]

1 species

Gyrosmilia interrupta (Ehrenberg 1834)

Heterocyathus Milne Edwards \& Haime 1848

(Arabian Gulf [9], South Africa [8], Japan [9, 44]; probably widely distributed in the Indo-Pacific. May be abundant on soft substrata around deeper reefs [75])

Eleven nominal species, an unknown number of valid species [75]

Heterocyathus aequicostatus Milne Edwards \& Haime 1848

Heterocyathus alternatus Verrill 1865

Heterocyathus eupsammides (Gray 1849)

Heterocyathus sulcatus (Verrill I866) 
Holcotrochus Dennant 1902

11

Known only from Australia [75]

Two species have been recorded. Deep water

Holcotrochus crenulatus Dennant 1904

Holcotrochus scriptus Dennant 1902

Hoplangia Gosse 1860

(Mediterranean, North-east Atlantic [107]. 300-2,600m depth)

Possibly only a single species [107]

Hoplangia durotrix Gosse 1860

Idiotrochus Wells 1935/6?

(West Pacific, Japan. 82-645m)

2 species [23]

Idiotrochus kikutii (Yabe \& Eguchi 1941)

Idiotrochus perexigua (Dennant 1906)

Kionotrochus Dennant 1906

I species

Kionotrochus suteri Dennant 1906

Labyrinthocyathus Cairns 1979

II

(Western Atlantic; Madagascar; New Zealand [10]. Approximately 385-810m depth)

At least 4 species

Labyrinthocyathus cornu (Gardiner 1904)

Labyrinthocyathus facetus Cairns 1979

Labyrinthocyathus kondoi (Wells 1977)

Labyrinthocyathus langi Caims 1979 ?langae

Labyrinthocyathus limatulus (Squires 1964)

Labyrinthocyathus quaylei (Durham 1947)

Lochmaeotrochus Alcock 1902

1 species

II

Lochmaeotrochus oculeus Alcock 1902

Lophelia Milne Edwards \& Haime 1849

II

(Mediterranean, Atlantic Ocean [107], South Africa [8]. Approximately 60-2,170m depth)

There is possibly only a single, widely distributed species [25]

Lophelia pertusa (Linnaeus 1758) 
Known only from the Lacapede Islands, north-western Australia [75]

One species, known from only a single specimen. Sometimes included in Trachyphylliidae [32].

\section{Montigyra kenti Matthai 1928}

Nemenzophyllia Hodgson \& Ross 1981

II

East Africa; Philippines [75]

1 species

Nemenzophyllia turbida Hodgson \& Ross 1981

Nomlandia Durham \& Barnard 1953

II

(Pacific Ocean [1a])

1 species

Nomlandia californica Durham \& Barnard 1953

Notocyathus Tenison-Woods 1880

Il

(West Pacific. 34-923m depth [23])

2 species

Notocyathus conicus (Alcock 1902)

Notocyathus venustus (Alcock 1902)

Odontocyathus Moseley 1881

?4 species

Odontocyathus coronatus (Pourtalès 1881)

Oryzotrochus Wells 1959

(Murray Islands, Australia [75]. Shallow water)

1 species

Oryzotrochus stephensoni Wells 1959

Oxysmilia Duchassaing 1870

(Caribbean and Bahamas; Gulf of Mexico [10]. Depth 46-640m)

1 specics

Oxysmilia portoricensis (Vaughan 1901)

Oxysmilia rotundifolia (Milne Edwards \& Haime 1849)

Paraconotrochus Cairns \& Parker 1992?

1 species

Paraconotrochus zeidleri 

40-540m

Two species recorded from North-East Atlantic [107]; four species from Australia [75]; six species from E. Pacific [25]. 12 species listed here, possibly more are valid

\section{Paracyathus arcuatus Lindström 1877}

Paracyathus calthus Verrill 1869

Paracyathus clathra Verrill 1869

Paracyathus conceptus Gardiner \& Waugh 1938

Paracyathus humilis Verrill 1870

Paracyathus indicus Duncan

Paracyathus molokensis Vaughan 1907

Paracyathus montereyensis Durham 1947

Paracyathus porphyreus Alcock 1904

Paracyathus profundus Duncan

Paracyathus pulchellus (Philippi 1842)

Paracyathus stearnsi Verrill 1869

Paracyathus stokesi Milne Edwards \& Haime

Paracyathus tiburonensis Durham 1947

Paracyathus vittatus Dennant 1906

Parasmilia Milne Edwards \& Haime 1848 = ?Coenosmilia/Dasmosmilia

2 species

II

II

Peponocyathus Gravier 1915

(Cosmopolitan [75]. Shallow to very deep water [10, 107])

Peponocyathus australiensis (Duncan 1870)

Peponocyathus folliculus (Pourtalès 1868)

Peponocyathus orientalis (Duncan 1876)

Peponocyathus stimpsonii (Pourtalès 1871)

Phacelocyathus Caims 1979

11

(Western Caribbean; Gulf of Mexico, Brazil [10]. Approximately 22-560m depth)

An unknown number of species.

Phacelocyathus flos (Pourtalès 1878)

Physogyra Quelch 1884

II

(Red Sea [64], East Africa [43]. Indian Ocean, north to Maldives and Andaman and Nicobar Islands [59]; south to Madagascar and north-western Australia [80]. Southeast Asia. Pacific Ocean, north to Ryukyu Islands, Guam, Marshall Islands and Phoenix Islands; south to Great Barrier Reef and New Caledonia; east to Samoa [75])

Countries listed without reference numbers are within the distribution range shown in Veron [75]

American Samoa [50]; Australia; British Indian Ocean Territory [65, 95]; Brunei; Christmas Island; Djibouti; Egypt [64]; Ethiopia; Federated States of Micronesia; Fiji; Guam; India [58, 59]; Indonesia; Israel [64]; Jordan [64]; Japan [78a]; Kenya [43]; Kiribati; Madagascar [56, 57]; Malaysia [4]: including Sabah [101]; Maldives [20, 65]; Marshall Islands; Mauritius; Myanmar; New Caledonia [102]; Northern Marianas; Palau; Papua New Guinea; Philippines [79]; Pitcairn Islands [54]; Qatar; Reunion; Samoa; Saudi Arabia [1b]; Seychelles [65, 99]; Singapore; Solomon Islands; Somalia; Sudan [64]; Tanzania [43]; Taiwan [34]; Thailand [36, 72]; Tokelau; Tuvalu; Vanuatu [76]; Wallis and Futuna; Yemen 
At least 2 valid species

Physogyra exerna Nemenzo \& Ferraris 1982

Physogyra gravieri Vaughan 1907

Physogyra lichtensteini (Milne Edwards \& Haime 1851)

Physogyra somaliensis Vaughan 1907

Platycyathus Fromentaal 1863

? species

Platytrochus Milne Edwards \& Haime 1848

II

(Indo-Pacific [75]. Shallow to deep water)

Two species recorded from Australia [75]

Platytrochus compressus (Tenison-Woods 1878)

Platytrochus hastatus Dennant 1902

Platytrochus laevigatus Caims \& Parker

Platytrochus parseptatus Cairns \& Parker

Plerogyra Milne Edwards \& Haime 1848

II

(Red Sea [64], East Africa [43]. Indian Ocean, north to Maldives and Andaman Islands [59]; south to Madagascar and Ningaloo Reefs (north-west Australia) [80]. South-east Asia. Pacific Ocean, north to Ryukyu Islands, Northern Marianas and Marshall Islands; south to Great Barrier Reef and New Caledonia, east to Phoenix Islands [75])

Countries listed without reference numbers are within the distribution range shown in Veron [75]

American Samoa [50]; Australia; British Indian Ocean Territory [65, 95]; Brunei; Christmas Island; Djibouti; Egypt [64]; Ethiopia; Federated States of Micronesia; Fiji; Guam; India [58, 59]; Indonesia; Israel [64]; Jordan [64]; Japan [78a]; Kenya [43]; Kiribati; Madagascar [56, 57]; Malaysia [4]: including Sabah [101]; Maldives $[20,65]$; Marshall Islands; Mauritius; Myanmar; New Caledonia [102]; Northern Marianas; Palau; Papua New Guinea; Philippines [79]; Pitcairn Islands [54]; Qatar; Reunion; Samoa; Saudi Arabia [1b]; Seychelles [55, 99]; Singapore; Solomon Islands; Somalia; Sri Lanka; Sudan [64]; Tanzania [43]; Taiwan [34]; Thailand [36, 72]; Tokelau; Tuvalu; Vanuatu [76]; Wallis and Futuna; Yemen

Fairly common and widespread on reefs, although restricted mostly to vertical faces [100]. Collected for use live in aquaria, and as a curio.

At least 4 species

Plerogyra eurysepta Nemenzo 1960

Plerogyra simplex Rehberg 1892

Plerogyra sinuosa (Dana 1846)

Plerogyra turbida (Hodgson \& Ross 1982)

Polycyathus Duncan 1876

11

(Cosmopolitan [75]. Shallow water)

One species recorded from Mediterranean and North-East Atlartic [107]; one unnamed species from Australia [75]. 3 species described from the Indian Ocean and Philippines [73]; 2 species from E. Pacific [25]; 3 from the Red Sea [64]. 12 species listed here, probably others are valid

Polycyathus andamanensis Alcock

Polycyathus atlanticus Duncan 1876

Polycyathus conceptus Gardiner \& Waugh 1938

Polycyathus fuscomarginatus (Klunzinger 1879) 
Polycyathus furanaensis Verheij \& Borel Best 1987

Polycyathus hodgsoni Verheij \& Borel Best 1987

Polycyathus hondaensis (Durham \& Barnard 1952)

Polycyathus isabela Wells 1982

Polycyathus marigondoni Verheij \& Borel Best 1987

Polycyathus muellerae (Abel 1959)

Polycyathus palifera (Verrill ?)

Polycyathus pallidus (Klunzinger 1879)

Polycyathus senegalensis Chevalier

Polycyathus verrilli Duncan

\section{Pourtalosmilia Duncan 1884}

(Mediterranean, North-east Atlantic [107]. Approximately 200-300m depth)

Probably two species

Pourtalosmilia anthophyllites (Ellis \& Solander 1786)

Pourtalosmilia conferta Caims 1978

Premocyathus Yabe \& Eguchi 1942

11

(Cosmopolitan. Shallow to deep water [75])

Possibly 2 species

Premocyathus burchae Cairns 1984

?Premocyathus compressus Yabe \& Eguchi 1942

Premocyathus dentiformis (Alcock 1902)

Pseudocyathocenas Cairns 1991

II

(Galapagos 1slands. 91-183m [25])

Pseudocyathoceras avis (Durham \& Bamard 1952)

Rhizosmilia Caims 1978

11

(Western Atlantic [10]. Approximately 123-355m)

An unknown number of species

Rhizosmilia gerdae Caims 1978

Rhizosmilia maculata (Pourtalès 1874)

Solenosmilia Duncan 1873

(Atlantic, Indian Ocean, south-East Australia. Approximately 280-2,165m depth)

An unknown number of valid species

Solenosmilia variabilis Duncan 1873

Sphenotrochus Milne Edwards \& Haime 1848

(Cosmopolitan [75], including Antarctica [45]. 9-403m)

8 species [23]

Sphenotrochus andrewianus Milne Edwards \& Haime 1848

Sphenotrochus aurantiacus Marenzeller 1904

Sphenotrochus auritus Pourtalès 1874

Sphenotrochus excavatus Tenison Woods 1878 
Sphenotrochus gardineri Squires 1961

Sphenotrochus gilchristi Gardiner 1904

Sphenotrochus hancocki Durham \& Barnard 1952

Sphenotrochus ralphae Squires 1964

Stephanocyathus Seguenza 1864

(Cosmopolitan [75]. Approximately 229-1158 m depth)

Four species recorded from Curacao [10]; three species from the North-East Atlantic [107]; one species from Australia [75]. 10 valid species listed here

Stephanocyathus campaniformis (Maranzeller 1904)

Stephanocyathus coronatus (Pourtalès 1867)

Stephanocyathus crassus (Jourdan 1895)

Stephanocyathus diadema (Moseley 1876)

Stephanocyathus laevifundus Caims 1977

Stephanocyathus moseleyanus (Sclater 1886)

Stephanocyathus nobilis (Moseley 1873)

Stephanocyathus paliferus Cairns 1977

Stephanocyathus platypus (Moseley 1881)

Stephanocyathus spiniger von Marenzeller 1888

Tethocyathus Kūhn 1933

(Atlantic [1a] and probably wider distribution)

Unknown number of valid species [75]

Tethocyathus cylindraceus (Pourtalès 1878)

Tethocyathus minor Gardiner 1899

Tethocyathus recurvatus (Pourtalès 1878)

Tethocyathus variabilis Cairns 1979

Thalamophyllia Duchassaing 1870

(Curacao [10]. Mediterranean, North-east Atlantic [107]. Approximately 18-1,317m depth)

Thalamophyllia gasti (Döderlein 1913)

Thalamophyllia gombergi Caims 1979

Thalamophyllia riisei (Duchassaing \& Michelotti 1860)

Thrypticotrochus Caims 1989

Australia: Queensland; Philippines; South China Sea [23]

2 species

Thrypticotrochus multilobatus Caims 1989

Thrypticotrochus petterdi (Dennant 1906)

Trematotrochus Tenison-Woods 1879

(Australia, New Zealand and the Caribbean $[10,75]$. Shallow to deep water)

Trematotrochus alternans Cairns \& Parker 1991?

Trematotrochus corbicula (Pourtalès 1878

Trematotrochus hedleyi Dennant 1906

Trematotrochus verconis Dennant 1904 
Two species recorded from North-East Atlantic [107]; four from Australia [75]; two from Japan [44]; five from Hawaii [52]. 13 species listed, possibly 20 are valid

Trochocyashus aithoseptatus Cairns 1984

Trochocyathus fasciatus Cairns 1979

Trochocyathus fossulus Cairns 1979

Trochocyathus gardineri (Vaugban 1907)

Trochocyashus mauiensis (Vaugban 1907)

Trochocyathus mediterraneus Zibrowius 1980

Trochocyathus meridionalis Duncan 1870

Trochocyathus oahensis Vaughan 1907

Trochocyathus rawsonii Pourtalès 1874

Trochocyathus spinosocostatus Zibrowius 1980

Trochocyathus tenuicalyx (Vaughan 1907)

Trochocyathus victoriae Duncan 1870

Trochocyathus virgatus Alcock 1902

Tropidocyathus Milne Edwards \& Haime 1848

(Indo-West Pacific 68-522m)

3 species [23]

Tropidocyathus lessoni (Michelin 1842)

Tropidocyathus nascornatus Gardiner \& Waugh 1938

Tropidocyathus pileus (Alcock 1902)

Vaughanella Gravier 1915

(North-east Atlantic [107]. Approximately 825-1,600m depth)

Possibly only a single species

Vaughanella concinna Gravier 1915

Vaughanella margaritata (Jourdan 1895)

Family FLABELLIDAE Bourne 1905

Blastotrochus Milne Edwards \& Haime 1848

(Philippines 11-18m depth [23])

1 species

Blastotrochus nutrix Milne Edwards \& Haime 1848

Flabellum Lesson 1831

(Worldwide, including Antarctica [23] 22-3,200 m)

This genus was discussed by Zibrowius in 1974 [106]; his conclusions form the basis of the revision published by Cairns, 1989 [23], but with changes to generic/subgeneric status. Over 100 nominal species; probably 47 valid species.

Flabellum alabastrum Moseley 1373

Flabellum angiostomum Folkeson 1919

Flabellum angulare Moseley 1876

Flabellum aotearoa Squires 1964 
RL Ref

Flabellum apertum Moseley 1876

Flabellum areum Cairns 1982

Flabellum atlanticum Caims 1979

Flabellum australe Moseley 1881

Flabellum campanulatum Holdsworth 1862

Flabellum chunii Marenzeller 1904

Flabellum coalitum Marenzeller 1889

Flabellum conuis Moseley 1881

Flabellum curvasum Moseley 1881

Flabellum daphnense Durham \& Barnard 1952

Flabellum deludens Marenzeller 1904

Flabellum dens Alcock 1902

Flabellum elongatum Moseley 1881

Flabellum flexuosum Cairns 1982

Flagellum floridanum ?

Flabellum fragile Cairns 1977

Flabellum gardineri Cairns 1982

Flabellum gracile Studer 1878

Flagellum hoffmeisteri Caims \& Parker

Flabellum impensum Squires 1962

Flabellum japonicum Moseley 1881

Flabellum knoxi Ralph \& Squires 1962

Flabellum lamellulosum Alcock 1902

Flabellum latum Studer 1878

Flabellum lowekeyesei Squires \& Ralph 1965

Flabellum macandrewi Gray 1849

Flabellum magnificum Marenzeller 1904

Flabellum marcus Keller 1974

Flabellum marenzelleri Cairns 1989

Flabellum messum Alcock 1902

Flabellum mortensi Studer 1877

Flabellum moseleyi Pourtalès 1880

Flabellum nobile Holdsworth 1862

Flabellum ongulense Eguchi 1965

Flabellum patens Moseley 1881

Flabellum pavoninum Lesson 1831

Flabellum planus Squires 1962

Flabellum politum Cairns 1989

Flabellum raukawaensis Squires \& Keyes 1967

Flabellum rubrum (Quoy \& Gaimard 1833)

Flabellum sexcostatum Cairns 1989

Flabellum sibogae Gardiner 1904

Flabellum spinosum Milne Edwards \& Haime 1848

Flabellum thouarsii Milne Edwards \& Haime 1848

Flabellum transversale Moseley 1881

Flabellum tubuliferum Tenison Woods 1880

Flabellum tuthilli Hoffmeister 1933

Flabellum vaughani Cairns 1984 
(Caribbean; Antarctic, South Africa; Philippines; Hawaii 2-700m depth [23])

Probably 8 species, but $G$. antarctica is considered more closely allied to the caryophlliid genera Crispatotrochus, Conotrochus or Labyrinthocyathus by Caims [23]

Gardineria antarctica Gardiner 1929

Gardineria capensis (Gardiner 1904)

Gardineria hawuiiensis Vaughan 1907

Gardineria minor Wells 1973 BZ

Gardineria musorstomica Cairns 1989

Gardineria paradoxa (Pourtales 1868)

Gardineria philippinensis Cairns 1989

Gardineria simplex (Pourtalès 1878)

Javania Duncan 1876

(Worldwide, including Antarctica [23] 52-3,165m depth)

5 named species, plus 2 undescribed species [23]

Javania antarctica (Gravier 1914)

Javania cailleti (Duchassaing \& Michelotti 1864)

Javania insignis Duncan 1876

Javania lamprotichum (Moseley 1880)

Javania pseudoalabastra Zibrowius 1974

Monomyces Ehrenberg 1834

(Cosmopolitan [75]. Shallow water)

Probably 2 recent species [23]

Monomyces anthophyllum Ehrenberg 1834

Monomyces pygmaea (Risso 1826)

Monomyces rubrum (Quoy \& Gaimard 1833)

Placotrochides Alcock 1902

(Caribbean; off Morocco; Indonesia; Philippines [23] 275-1628m depth)

This genus has customarily been considered as a junior synonym of Flabellum [23]. Possibly ?4 species

Placotrochides alabastrum (Alcock 1902)

Placotrochides frustra Caims 1979

Placotrochides scaphula Alcock 1902

Placotrochus Milne Edwards Haime 1848

(Indo-Pacific [4, 46]. Deep water)

6 nominal species; possibly 3 valid species

Placotrochus fuscus Vaughan 1907

Placotrochus laevis Milne Edwards \& Haime 1848

Placotrochus pedicellatus Tenison-Woods 1879

Caims [23] considers $P$. pedicellasus should be assigned to a separate genus) 
4 species [25]

Polymyces fragilis (Pourtalès 1868)

Polymyces montereyensis (Durbam 1947)

Polymyces tannerensis (Durham \& Bamard 1952)

Polymyces wellsi Caims 1991

Rhizotrochus Milne Edwards \& Haime 1848

(Indo-West Pacific 20-1,048m depth)

Probably 5 species [23]

Rhizotrochus flabelliformis Cairns 1989

Rhizotrochus levidensis Gardiner 1899

Rhizotrochus niinoi Yabe \& Eguchi 1942

Rhizotrochus radiatus Dennant 1904

Rhizotrochus typus Milne Edwards \& Haime 1848

Truncatoflabellum Cairns 1989

II

(Indo-Pacific, including New Zealand. Possibly Cape Verde 1slands 0-1163m depth [23])

Erected by Caims [23] to replace part of the Flabellum group described by Zibrowius [106]. About 25 species

Truncatoflabellum aculeatum (Milne Edwards \& Haime 1848)

Truncatoflabellum bairdi (Milne Edwards \& Haime 1848)

Truncatoflabellum candeanum (Milne Edwards \& Haime 1848)

Truncatoflabellum carinatum Caims 1989

Truncatoflabellum compressum (Lamarck 1816)

Truncatoflabellum corbicula (Tenison-Woods 1880)

Truncatoflabellum crassum (Milne Edwards \& Haime 1848)

Truncatoflabellum cumingii (Milne Edwards \& Haime 1848)

Truncatoflabellum formosum Cairns 1989

Truncatoflabellum gippslandicus (Dennant 1889)

Truncatoflabellum inconstans (Marenzeller 1904)

Truncatoflabellum incrustatum Cairns 1989

Truncatoflabellum irregulare (Semper 1872)

Truncatoflabellum martensii (Studer 1878)

Truncatoflabellum paripavoninum (Alcock 1894)

Truncatoflabellum profundum (Milne Edwards \& Haime 1848)

Truncatoflabellum pusillum Caims 1989

Truncatoflabellum spheniscus (Dana 1846)

Truncatoflabellum stabile (Marenzeller 1904)

Truncatoflabellum stokesi (Milne Edwards \& Haime 1848)

Truncatoflabellum trapezoideum (Keller 1981)

Truncatoflabellum truncum (Cairns 1982)

Truncatoflabellum variabile of Gerth 1921

Truncatoflabellum victoriae (Duncan 1865) 
Family GUYNIIDAE Hickson 1910

Guynia Duncan 1872

11

(Atlantic Ocean; Australia: south; Persian Gulf; Red Sea; New Caledonia; Philippines; USA(Hawaiian Islands) $28-653 \mathrm{~m}[23])$

1 species [23]

Grynia annulata Duncan 1872

Pourtalocyathus Caims 1979

II

(Western Atlantic 349-1200m depth [10])

Total number of species unknown; one recorded from Curacao [10]

Pourtalocyathus hispidus (Pourtalès 1878)

Schizocyathus Pourtalès 1874

II

(Western and North-east Atlantic [107]. Approximately 88-1,300m depth)

Probably a single species

Schizocyathus fissilis Pourtalès 1874

Stenocyathus Pourtalès 1871

II

(Cosmopolitan [16, 107], including Antarctica [105]. Approximately 110-1229m depth)

I species

Stenocyathus vermiformis (Pourtalès 1868)

Truncatoguynia Caims 1989

(South China Sea off Hong Kong; Kermadec Ridge. About $160 \mathrm{~m}$ depth)

One described species; one undescribed [23]

Truncatoguynia irregularis Cairns 1989

Family DENDROPHYLLIDAE Gray 1847

Astroides Quoy \& Gaimard 1827

I1

(Mediterranean [107]. Shallow water)

1 species

Astroides calycularis (Pallas 1766)

Astropsammia Verrill 1869

1 species

11

Astropsammia pedersenii Verrill 1869 
Balanophyllia S.V. Wood 1844

Approximately 28 valid species

Balanophyllia affinis Semper

Balanophyllia bairdiana Milne Edwards \& Haime 1848

Balanophyllia bayeri Cairns 1979

Balanophyllia buccina Tenison-Woods 1878

Balanophyllia caribbeana Cairns 1977

Balanophyllia caulifera?

Balanophyllia cellulosa Duncan 1873

Balanophyllia chnous Squires 1962

Balanophyllia cornu Moseley 1881

Balanophyllia cumingii Milne Edwards \& Haime

Balanophyllia cyathoides (Pourtalès 1871)

Balanophyllia dentata Tenison-Woods 1879.

Balanophyllia desmophylloides Vaughan 1907

Balanophyllia diffusa Harrison \& Poole 1909

Balanophyllia dilatata Dennant 1904

Balanophyllia dineta Cairns 1977

Balanophyllia diomedeae Vaughan 1907

Balanophyllia eguchii Wells 1982

Balanophyllia elegans Verrill 1864

Balanophyllia europaea (Risso 1826)

Balanophyllia floridana Pourtalès 1868

Balanophyllia galapagensis Vaughan 1906

Balanophyllia gemmifera Klunzinger 1879

Balanophyllia grandis Cairns 1977

Balanophyllia hadros Cairns 1979

Balanophyllia imperialis Kent

Balanophyllia incisa Crossland 1952

Balanophyllia italica Michelin

Balanophyllia laysanensis Vaughan 1907

Balanophyllia malouinensis Squires 1961

Balanophyllia palifera Pourtalès 1878

Balanophyllia rediviva Moseley 1881

Balanophyllia regia Gosse 1860

Balanophyllia scabra Alcock

Balanophyllia scabrosa (Dana 1846)

Balanophyllia thalasse Zibrowius 1980

Balanophyllia verrucaria Pallas

Balanophyllia wellsi Caims 1977

Balanophyllia yongei Crossland 1952

Bathypsammia Marenzeller 1907/6?

(Western Atlantic 210-1,079m depth)

Probably 2 species

Bathypsammia fallosocialis Squires 1959

Bathypsammia tintinnabulum (Pourtalès 1868) 
Cladopsammia Lacaze-Duthiers 1897

I]

(Not fully known, but reported from the Mediterranean [107], Australia [75] and Hawaii [52]. Shallow water)

Probably 2 species

Cladopsammia echinata Cairns 1984

Cladopsammia rolandi Lacaze-Duthiers 1897

Coenopsammia Milne Edwards \& Haime 1848

? species

Coenopsammia equiserialis Milne Edwards 1857

Coenopsammia radiata Verrill 1864

Coenopsammia tenuilamellasa Milne Edwards \& Haime 1848

Dendrophyllia Blainville 1830

(Cosmopolitan, including Antarctica [45]. Reefs and rocks, shallow to deep water)

Possibly around 30 valid species; 21 listed below.

Dendrophyllia alcocki (Wells 1954)

Dendrophyllia alternata Pourtalès 1880

Dendrophyllia arbuscula Horst 1922

Dendrophyllia atrata Dennant 1906

Dendrophyllia californica Durham 1947

Dendrophyllia coarctata Duncan

Dendrophyllia cornigera (Lamarck 1816)

Dendrophyllia cornucopia Pourtalès 1871

Dendrophyllia danae Verrill 1872

Dendrophyllia fistula Alcock 1902

Dendrophyllia gaditana (Duncan 1873)

Dendrophyllia gracilis Milne Edwards \& Haime 1848

Dendrophyllia hannibali Nomland 1916

Dendrophyllia horsti Gardiner \& Waugh 1939

Dendrophyllia indica Pillai

Dendrophyllia johnsoni Caims 1991

Dendrophyllia laboreli Zibrowius \& Brito 1984

Dendrophyllia micranthus (Ehrenberg)

Dendrophyllia minuscula Bourne 1905

Dendrophyliia nigrescens Dana 1846

Dendrophyllia oahensis Vaughan 1907

Dendrophyllia oldroydi Faustino 1931

Dendrophyllia praecipua Gardiner \& Waugh 1939

Dendrophyllia ramea (Linnaeus 1758)

Dendrophyllia robusta Bourne 1905

Dendrophyllia serpentina Vaughan 1907

Dendrophyllia velata Crossland 1952

\section{Duncanopsammia Wells 1936}

(South-western Australia, north to north coast of Australia and New Guinea; south to Great Barrier Reef (eastern Australia) [75])

A small, rare coral, generally occurring in water depths over 20 metres [75]. 1 species

Duncanopsammia axifuga (Milne Edwards \& Haime 1848) 
Enallopsammia Michelotti 1871 11

(Cosmopolitan [10, 107]. Approximately 229-2,165m depth)

Five recent species [107]

Enallopsammia amphelioides (Alcock 1902)

Enallopsammia marenzelleri Zibrowius 1973

Enallopsammia profunda (Pourtalès 1867)

Enallopsammia pusilla (Alcock 1902)

Enallopsammia rostrata (Pourtalès 1878)

Endopachys Lonsdale 1845

(Indo-Pacific, from Africa [8] to Australia [75], Japan [44] and USA: California [69])

An unknown number of species (possibly 6)

Endopachys australiae Tenison-Woods 1878

Endopachys grayi Milne Edwards \& Haime 1848

Endopachys maclurii Milne Edwards \& Haime 1848

Endopsammia Milne Edwards \& Haime 1848

(Indo-Pacific, South Atlantic and Mediterranean [75])

II

An unknown number of species (possibly 5)

Endopsammia philippinensis Milne Edwards \& Haime 1848

Endopsammia pourtalesi (Durham \& Barnard 1952)

Eupsammia Milne Edwards \& Haime 1848

?3 species

Heteropsammia Milne Edwards \& Haime 1848

(Red Sea, Persian Gulf. East and South Africa [8, 43]. Indian Ocean, north to Arabian Gulf [9], Gulf of Mannar (southern India) and Andaman and Nicobar Islands [59]; south to Madagascar and south-western Australia [75]. South-east Asia. Pacific Ocean, north to southern Japan [103]; south to New Guinea and to Sydney, south-tast Australia [75])

Countries listed without reference numbers are within the distribution range shown in Veron [75]

Australia; Bahrain [9. 67]; British Indian Ocean Territory [65, 95]; Brunei; China [111, 112]; Christmas Island; Cocos (Keeling) Islands [77]; Comoros; Djibouti; Egypt [64]; Ethiopia; Hong Kong [64a]; India [58, 59]; Indonesia; Iran; Israel [64]; Jordan [64]; Japan [78a]; Kenya [43]; Kuwait; Madagascar [56, 57]; Malaysia [4, 101]; Maldives [20, 65]; Mauritius; Mozambique [8]; Myanmar; Oman [66]; Palau; Papua New Guinea; Philippines [79]; Qatar; Reunion; Saudi Arabia [1b]; Seychelles [65, 99]; Singapore; Somalia; Sri Lanka; Sudan [64]; Tanzania [43]; Taiwan [34]; Thailand [36, 72]; United Arab Emirates [9, 67]; Yemen

Nine nominal species; probably two species [46]

Heteropsammia cochlea (Spengler 1781)

Heteropsammia eupsammides (Gray 1849)

Heteropsammia moretonensis Wells 1964 
Leptopsammia Milne Edwards \& Haime 1848

(Mediterranean; North-east Atlantic; Australia; Indonesia [75, 100]. Shallow to deep water)

At least five species.

Leptopsammia britannica (Duncan 1870)

Leptopsammia chevalier Zibrowius 1980

Leptopsammia formosa (Gravier 1915)

Leptopsammia pruvoti Lacaze-Duthiers 1897

Leptopsammia queenslandiae Wells 1964

Leptopsammia stokesiana Milne Edwards \& Haime 1848

Leptopsammia trinitatis Hubbard \& Wells 1986

\section{Notophyllia Dennant 1849}

(Known only from south-eastern Australia [75]. Shallow to deep water)

Three species have been recorded [75]

Notophyllia etheridgei Hoffmeister 1933

Notophyllia recta Dennant 1906

Notophyllia variolaris (Tenison-Woods 1877)

Psammoseris Milne Edwards \& Haime 1851

(lndo-Pacific)

Total number of species unknown; one has been recorded from Australia [75].

Psammoseris hemisph(a)erica (Gray 1850)

Rhizopsammia Verrill 1869

II

(Atlantic [107]; Indo-Pacific: including South Africa [8]. Approximately 55-135m depth)

At least 5 species

Rhizopsammia chamissoi Wells 1954

Rhizopsammia compacta Sheppard \& Sheppard 1991

Rhizopsammia manuelensis Chevalier 1966

Rhizopsammia minuta?

Rhizopsammia pulchra Verrill 1869

Rhizopsammia verrilli van der Horst 1922

Rhizopsammia wellingtoni Wells 1982

Rhizopsammia wettsteini Scheer \& Pillai 1983

Thalassiotrochus Milne Edwards 1861

11

(Mediterranean about $2000 \mathrm{~m}$ depth)

One species described, which could be a young Desmophyllum [107]

Thalassiotrochus telegraphicus Milne Edwards 1861

Thecopsammia Pourtalès 1868

(Cosmoplitan $[10,75]$ )

An unknown number of species (possibly 6) 
(Caribbean [96]. Red Sea [64], Arabian Gulf [9]. Indian Ocean, north to Gulf of Kutch (north-west India) and Andaman and Nicobar Islands [59]; south to Madagascar and south-western Australia [31]. South-east Asia. Pacific Ocean, north to Japan [40], south to southern Australia [75]; east to California [38, 69])

American Samoa [50]; Anguilla; Antigua and Barbuda; Australia; Bahrain [9, 16]; Barbados; Brazil [49]; British Indian Ocean Territory [65, 95]; British Virgin Islands [37]; Brunei; Cayman Islands; Chile; China [111, 112]; Christmas Island; Cocos (Keeling) Islands [77]; Colombia [62]; Comoros; Cook Islands; Costa Rica [33]; Cuba [48]; Djibouti; Dominica; Dominican Republic; Ecuador; Egypt [64]; Ethiopia; Federated States of Micronesia; Fiji; French Polynesia [30, 42]; Grenada; Guadeloupe; Guam; Haiti; Hawaiian Islands [52]; Honduras; Hong Kong [64a]; India [58, 59]; Indonesia; Iran; Israel [64]; Jamaica [96]; Japan [78a]; Jordan [64]; Kenya [43]; Kiribati; Kuwait; Madagascar [56, 57]; Malaysia [4]: including Sabah [101]; Maldives [20, 65]; Marshall Islands; Martinique; Mauritius; Montserrat; Mozambique [8]; Myanmar; Nauru; Netherlands Antilles [47]; New Caledonia [102]; New Zealand; Nicaragua; Niue; Northern Marianas; Oman [66]; Palau; Panama [61]; Papua New Guinea; Philippines [79]; Pitcairn Islands [54]; Puerto Rico; Qatar; Reunion; Saint Kitts and Nevis; Saint Lucia; Saint Vincent and the Grenadines; Samoa; Saudi Arabia [1b]; Seychelles [65, 99]; Singapore; Solomon Islands; Somalia; Sri Lanka; Sudan [64]; Tanzania [43]; Taiwan [34]; Thailand [36, 72]; Tokelau; Tonga; Trinidad and Tobago; Turks and Caicos; Tuvalu; United Arab Emirates [9, 67]; United States: California [52]; Vanuatu [76]; Virgin Islands of the United States; Venezuela; Wallis and Futuna; Yemen

Tubastraea is usually found in caves on reefs, except for $T$. micrantha, which occurs on reefs faces exposed to currents. Collected for use as a curio. There is one cosmopolitan species, T. coccinea (the only species in the Western Atlantic) [41]. This species and three others are recognised from Australia, but the valid number of species worldwide is unknown [75]

Tubastraea aurea (Quoy \& Gaimard 1833)

Tubastraea coccinea Ehrenberg 1834

Tubastraea diaphana Dana 1846

Tubastraea faulkneri Wells 1982

Tubastraea floreana Wells 1982

Tubastraea micrantha (Ehrenberg 1834)

Tubastraea tagusensis Wells 1982

\section{Turbinaria Oken 1815}

(Red Sea [64], Persian Gulf, East and South Africa [8, 43]. Indian Ocean, north to Arabian Gulf [9], Gulf of Kutch (north-west India) and Andaman and Nicobar Islands [59]; south to Madagascar and south-western tip of Australia [31]. South-east Asia. Pacific Ocean, north to southern Japan [40], Marshall Islands and Phoenix Islands; south to Lord Howe lsland and Kermadec lslands [75]; east to Tubuai Islands [42])

Countries listed without reference numbers are within the distribution range shown in Veron [75]

American Samoa [50]; Australia; Bahrain [9. 67]; British Indian Ocean Territory [65, 95]; Brunei; China [111, 112]; Christmas Island; Cocos (Keeling) Islands [77]; Comoros; Cook Islands; Djibouti; Egypt [64]; Ethiopia; Federated States of Micronesia; Fiji; French Polynesia [30, 42]; Guam; Hong Kong [64a]; India [58, 59]; Indonesia; Iran; Israel [64]; Jordan [64]; Japan [78a]; Kenya [43]; Kiribati; Kuwait; Madagascar [56, 57]; Malaysia [4]: including Sabah [101]; Maldives [20, 65]; Marshall Islands; Mauritius; Mozambique [8]; Myanmar; Nauru; New Caledonia [102]; New Zealand: Kermadec Islands; Niue; Northern Marianas; Oman [66]; Palau; Papua New Guinea; Philippines [79]; Pitcairn Islands [54]; Qatar; Reunion; Samoa; Saudi Arabia [1b]; Seychelles [65, 99]; Singapore; Solomon Islands; Somalia; Sri Lanka; Sudan [64]; Tanzania [43]; Taiwan [34]; Thailand [36, 72]; Tokelau; Tonga; Tuvalu; United Arab Emirates [9, 67]; Vanuatu [76]; Wallis and Futuna; Yemen 
Widely distributed on reefs, occurring in many habitats [41]. Several species (e.g. T. heronensis and T. bifrons) are common on temperate reefs although uncommon in the tropics [75]. Collected for use as a curio. Eighty nominal species, 10 valid species from Australia, but an unknown number altogether [75], 11 are listed below

Turbinaria bifrons Brüggemann 1877

Turbinaria brassica (Dana 1846)

Turbinaria conspicua Bernard 1896

Turbinaria crater (Pallas 1766)

Turbinaria dance Bernard

Turbinaria elegans Bernard 1896

Turbinaria frondens (Dana 1846)

Turbinaria heronensis Wells 1958

Turbinaria irregularis Bernard 1896

Turbinaria marmorea Rehberg MV

Turbinaria mesenterina (Lamarck 1816)

Turbinaria mollis Bernard

Turbinaria pasula (Dana 1846)

Turbinaria peltata (Esper 1794)

Turbinaria porcellana Bernard

Turbinaria pulcherrima Bemard

Turbinaria radicalis Bernard 1896

Turbinaria reniformis Bernard 1896

Turbinaria schistica Gardiner 1898

Turbinaria sinensis Verrill 1866

Turbinaria stellulata (Lamarck 1816)

Turbinaria undata Bemard 1896 


\section{REFERENCES - HARD CORALS}

14. American Fisheries Society 1991. Common and scientific names of aquatic invertebrates from the United States and Canada: Cnidaria and Clenophora. American Fisherieg Society, Bethesda, Maryland.

Ib. Antonius A., Scheer, G. and Bouchon, C. 1990. Corals of the Eastern Red Sea. Atoll Res. Bull. No 334.

2. Best, M.B. and Hoeksems, B.W. 1987. New observations on scleractinian corals from Indonesia: 1. Free-living species belonging to the Faviins. Zool. Meded. 61 (27): 387-403.

3. Best, M.B. and Suharsono, ? 1991 New observations on Scleractinian corals from Indonesia: 3 . Species belonging to the Merulinidae with new records of Merulina and Boninastrea. Zool. Meded. 65: 333-342.

4. Betterton, C. 1981. A guide to the hard corals of Peninsular Malaysia (excluding the genus Acropora). Malayan Nat. J. 34: 171-336.

5. Boschms, H. 1948. The species problem in Millepara. Zool. Verh. 1: 3-115.

6. Boschms, H. 1962. On milleporine corals from Brazil. $K$. Ned. Acad. Wet. Amsterdam Proc. 65C: 302-312.

7. Boschms, H. 1966. On new species of Millepora from Mauritius with notes on the specific characte:s of $M$. exaesa. K. Ned. Acad. Wer. Amsterdam Proc. 69C: 409-419.

8. Boshoff, P.H. 1981. An annotated checklist of southern Africa Scleractinia. Invest. rep. Oceanogr. Res. Inst. 49: $1-45$.

9. Burchard, J.E. 1979. Coral fauna of the westem Arabian Gulf. 129pp. Arabian American Oil Co., Dhahran (Saudi Arabia).

10. Cairns, S.D. 1979. The deep-water Scleractinia of the Caribbean Sea and adjacent waters. Studies on the Fauna of Curaçao and other Caribbean Islands 57(180): $341 \mathrm{pp}$.

11. Cairns, S.D. 1982. Stony corals (Cnidaria: Hydrozoa, Scleractinia) of Carrie Bow Cay, Belize. pp 271 -302, in K. Rützler and 1.G Macintyre, eds., The Atlantic Barrier Reef Ecosystem at Carrie Bow Cay, Belize, 1: Structure and communities. Smithsanian Contributions to Marine Sciences 12: $539 \mathrm{pp}$.

12. Cairns, S.D. 1982. Antarctic and subantarctic Scleractinia. Antarctic Research Series 34: 1-74.

13. Cairns, S.D. 1983. Antarctic and subantarctic Stylasterina (Coelentaerata: Hydrozoa). Antarcric Research Series 38: 61-164.

14. Cairns, S.D. 1983. A generic revision of the Stylasterina (Coelenterata: Hydrozos). Part 1. Description of the genera. Bull. Mar. Sci. 33 (2): 427-508.

15. Caims, S.D. 1983. Pseudocrypthelia, a new genus of Stylasterine coral (Coelenterata: Hydrozoa) from the Indonesisn region. Beaufortia 33(3): 29-35.

16. Caims, S.D. 1984. New records of ahermatypic corals (Scleractinia) from the Hawaiian and Line Islands. Occasional Papers of the Bemice P. Bishop Musewm 25(10): 1-30.
17. Caims, S.D. 1985. Three new species of Stylasteridae (Coelenterata: Hydrozoa). Proc. Biol. Soc. Wash. 98 (3): 728-739.

18. Caims, S.D. 1986. A reviaion of the Northwest Atlantic Stylasteridae. Smithsonian Cantributions ta Zoology 418: 131 pages.

19. Cairns, S.D. 1986. Stylasteridae (Hydrozoa: Hydroida) of the Galapagoo 1slands. Smithsanian Contributions to Zoology 426: 42pp.

20. Cairns, S.D. 1987. Conopara adeta, new species from Australia, the firat known unattached Stylasterid. Proc. Biol. Soc. Washington 100(1): 141-146.

21. Caims, S.D. 1987. Range extensions of ahermatypic scleractinia in the Gulf of Mexico. Northeast Gulf Science 9: 131-134.

22. Cairns, S.D. 1988. New records of Stylasteridse (Cnidaria: Hydrozoa) from Westem Australia, including the description of two new species. Rec. West. Aust. Mus. 14(1): $105-119$.

23. Cairns, S.D. 1989. A revision of the ahermatypic scleractinia of the Philippine lslands and adjacent waters, Part 1: Fungiacyathidae, Micrabaciidae, Turbinoliniiae, Guyniidae and Flabellidae. Smithsonian Contriburions to Zoology $\mathrm{N}^{\circ} 486$.

24. Cairns, S.D. 1990. Antarctic Scleractinia, Vol 1, in J.W. Wägela \& J. Sieg, eds., Synopses of the Antarcric Benthos. Koenigstein, Koeltz Scientific Books.

25. Cairns, S.D. 1991. A revision of the ahermatypic Scleractinia of the Galapagos and Cocos Islands. Smithsonian Cantributions to Zoology N ${ }^{\circ} 504$

26. Cairns, S.D. 1991. A generic revision of the Stylasteridae (Coelenterata: Hydrozoa). Part 3: Keys to the genera. Bull. Marine Science 49(1-2): 538-545.

27. Cairns S.D. 1991. The Marine Fauns of New Zealand: Stylasteridae (Cnidaria: Hydroida). New Zealand Oceanagraphic Insritute Memoir 98.

28. Cairns S.D. 1991. Cyclohelia lamellata, new genus and species of Stylasteridae (Cnidaria: Hydrozoa) from the Bering Sea. Pacific Science 45 (4): 383-388.

29. Caims S.D. 1991. Catalog of the type specimens of stony corals (Milleporidae, Stylasteridae, Scleractinia) in the National Museum of Natural History, Smithsonian Institution. Smithsanian Contriburions to Zoology No 514.

30. Chevalier, J-P. 1979. La faune corallienne (Scléractiniaires et Hydrocoralliaires) de la Polynésie française. Cah. Indo-Pacif. 1: 129-151.

31. Chevalier, J-P. 1980. La faune corallienne de l'île Tubuaī (Archipel des Australes). Cah. Indo-Pacif. 2(3): 55-68.

32. Chevalier, J-P. and Beauvais, L. 1987. Ordre des Scléractiniaires, 11, Systématique, p. 679-764. In D. Doumenc (ed.): Traisé de Znologie 3 (3). Mssson, Poris.

32a. Clae reboudt, M. 1990. Galarea paucisepta nom. nov. (for G. pauciradiata), rediscovery and redescription of a poorly known scleractinian species (Oculinidse). Galarea 9: 1-8. 
32b. Claereboudt, M. and Hoeksema, B.W. 1987. Fungia (Verillofungia) spinifer spec. nov., a new scleractinian coral (Fungiidae) from the Indo-Malayan region. Zool. Meded. 61: 303-309.

33. Conés, J. 1990. The coral reefs of Golfo Dulce, Costs Rica: distribution and community structure. Atoll Res. Bull. $N^{*} 344$.

33a. Cubit, J. and Williams, S. 1983. The invertebrates of Galeta Reef (Caribbean Panama): a species list and bibliography. Atoll Res. Bull. 269.

34. Dai, C-F. 1991. Reef environment and coral fauna of Southem Taiwan. Atoll Res. Bull. N 354.

35. Dinesen, Z.D. 1980. A revision of the coral genus Leptoseris (Scleractinia: Fungiina: Agariciidae). Mem. Queenst. Mus. 20: 181-235.

36. Ditlev, H. 1976. Stony corals (Coelenterata: Scleractinia) from the west coast of Thailand. Phuket Marine Biological Center, Research Bullerin No. 13:14 pp.

36a. Ditler, H. 1980. A field guide to the reef-building corals of the Indo-Pocific. Backhuys, Rotterdam \& Scandinavian Science Press, Klampenborg.

37. Dunne, R.P. and Brown B.E. 1979. Some aspects of the ecology of reefs surrounding Anegada. British Virgin Islands. Atoll Res. Bull, 236.

38. Durham, J.W. 1962. Corals from the Galapagos and Cocos Islands. Proc. Calif. Acad. Sci. (4)32: 41-56.

40. Eguchi, M. and Miyawaki, T. 1975. Systematic study of the scleractinian corals of Kushimoto and its vicinity. Bull. Mar. Park Res. Sins. 1: 47-62.

41. Faure, G. 1977. Annotated check list of corals in the Mascarene Archipelago, Indian Ocean. Atoll Res. Bull. N $^{\circ}$ 203: 1-26.

42. Faure, G. 1985. Faune corallienne des lles Rapa et Marotiri, Polynesse Française Iles Australes. Prac. Sth Int. Coral Reef Congress, Tahiri. 6: 267-272.

42a. Grigg, R.W. 1981. Acropora in Hawaii, 2. Zoogeography. Pac. Sci. 35: 15-24.

43. Hamilton, H.G.H, and Brachel, W.H. 1984. Structure and coral fauna of East African reefs. Bull. Mar. Sci. 34: 248-266.

39. Head, S.M. 1983. An undescribed species of Merulina and a new genus and species of siderastreid coral from the Red Sea. Joumal of Natural History. 17: 419-435.

44. Hoeksems, B.W. 1989. Taxonomy, phylogeny and biogeography of mushroom corals (Scleractinia: Fungiidae). Zool. Verh. 254: 1-295.

45. Hocksema, B.W. and Best, M.B. 1984. Cantharellus noumeae (gen. nov., spec. nov.), a new scleractinian coral (Fungiidac) from New Caledonia. Zool. Meded. 58(19): 323-328.

46. Hoeksema, B.W. and Best, M.B. 1991. New observations on scleractinian corals from Indonesia: 2 Sipunculan-associated species belonging to the genera Heterocyathus and Heteropsammia. Zool. Meded. 65: 221-245.

47. Jaap, W.C., Lyons, W.G., Dustan, P. and Halas, J.C. 1989. Stony coral (Scleractinia and Milleporina) community structure at Bird Key Reef, Ft. Jefferson National Monument, Dry Tortugas, Florida. Florida Marine Research Publicarions 46.
48. Kühimann, D.H.H. 1971. The coral reefs of Cuba. II. On the ecology of the bank reefs and their corals. Int. Revue ges. Hydrabiol. 56(2): 145-199.

49. Laborel, J. 1970. Madréporaires et hydrocoralliaires récifaux des cotes brésiliennes, systematique, ecologie, repartition verticale et geographique. Annls. Inst. Oceanogr. Paris 47 (1): 171-229.

50. Lamberts, A.E. 1983. An annotated check list of the corals of American Samoa. Atoll. Res. Bull. N 264.

51. Lewis, J.B. 1989. The ecology of Millepora. A review. Coral Reefs 8: 99-107.

52. Maragos, J.E. 1977. Order Scleractinia: stony corals. In: Davaney D.M. \& Eldredge L.G. (eds), Reef and Shore Fauna of Hawaii Section 1: Protozoa through Ctenophora. Bernice P. Bishop Mus. Special Pub. 64: 158-214.

53. Moll, H. and Best, M.B. 1984. New scleractinian corals (Anthozoa: Scleractinia) from the Spermonde Archipelago, South Sulawesi, Indonesia. Zool. Meded. 58(4): 47-58.

54. Paulay, G. 1989. Marine inventebrates of the Pitcairn Islands: species composition and biogeography of corals, molluses and echinoderms. Atoll Res. Bull. № 326.

55. Peters E.C., Cairns, S.D., Pilson, M.E.Q., Wells, J.W., Jaap, W.C., Lang, J.C., Cummings Vasleski, C.E. and Gollahon L. St. Pierre. 1988. Nomenclature and biology of Astrangia poculata $(=A$. danae, $=A$. astreiformis). (Cnidaria: Anthozoa). Proc. Biol. Soc. Wash. 10(2): 234-250.

56. Pichon, M. 1964. Contribution à l'étude de la repartition des Madréporaires sur le récif de Tuléar, Madagascar. Rec. Trov. Stat. Mar. Endaume-Marseille, fasc. hors. sér., suppl. 2: 79-203.

57. Pichon, M. 1978. Recherches sur les peuplements à dominance d'anthozoaires dans les récifs coralliens de Tuléar (Madagascar), Atoll Res. Bull. № 222.

57a. Pichon, M. 1980. Wellsophyllia radiata n. gen, n. so., a new hermatypic coral from the Indonesian region. (Calidaria, Anthozoa, Scleractinia). Rev. Swisse Zool. 87(1): 253-259.

58. Pillai, C.S.G. 1971. The distribution of shallow-water stony corals at Minicoy Atoll in the Indian Ocean with a check-list of species. Atoll Res. Bull. 141: 1-12.

59. Pillai, C.S.G. 1983. Structure and generic diversity of recent Scleractinia of India. J. Mar. Biol. Assoc. India 25: 78-90.

60. Pillai, C.S.G. and Scheer, G. 1976. Report on the stony corals from the Maldive Archipelago. Zoologica (Stuttgart) 43(126): 1-83.

61. Poner, J.W. 1972. Ecology and species diversity of coral reefs on opposite sides of the isthmus of Panama. Bull. Biol. Soc. Wash. 2: 89-116.

62. Prahl H.V. and Erhardt, H. 1985. Columbia Corales $y$ arrecifes Coralinos. Fen Columbia.

63. Ralph P.M. and Squires, D. 1962. The extant scleractinian corals of New Zealand. Zool. Publ. Victaria Univ. Wellington. 29: 1-19.

63a. Rosen, B.R. 1971. Annotated checklist and bibliography of corals of the Chagos Archipelago (including the recent collection from Diego Garcia), with remarks on their distribution. Atoll Res. Bull. 149: 67-88. 
63b. Rosen, B.R. 1979. Checklist of recent corals from Aldabra (Indian Ocean). Atoll Res. Bull. 233: 1-26.

63c. Scheer, G. and Pillai, C.S.G. 1974. Report on Scleractinia from the Nicobar lslands. Zoologica (Stuttgart) 42(122): 175 .

64. Scheer, G. and Pillai, C.S.G. 1983. Report on the stony corals from the Red Sea. Zoologica (Switgart) 45 (J33): $1-198$

64a. Scou, P.J.B. 1984. The Corals of Hong Kong. Hong Kong University Preas, Hong Kong.

65. Sheppard C.R.C. 1987. Coral species of the Indian Ocean and adjacent seas: a synonymised compilation and some regional distributional patterns. Atoll Res. Bull. 307: 32 pp.

66. Sheppard, C.R.C. and Salm, R.V. 1988. Reef and coral communitiea of Oman, with a description of a new coral species (Order Scleractinia, genus Acanthastrea). Joumal of Natural History 22: 263-279.

67. Sheppard, C.R.C. and Sheppard, A.L.S. 1991. Corals and coral communities of Arabia. Fauna of Saudi Arabia 12.

68. Smith, F.G.W. I971. Atlantic reef corols. University of Miami Press. J64pp.

69. Squires, D.F. 1959. Corals and coral reefs in the Gulf of California. Results of the Puritan-American Museum of Natural History Expedition to Western Mexico, Bull. Am. Mus. nat. Hist. 118(7): 367-431.

70. Thiel, M.E. 1928. Madreporia. Beitr. Kennt. Meeresfauna Westafr. 3 (6): 251-350.

71. Tribble, G.W. and Randall, R.H. 1986. A description of the high-latitude shallow water coral communities of Miyake-jims, Japan. Coral Recfs 4: 151-159.

72. Unesco 1985. Coral Taxonomy. Results and recommendations of a regional Unesco (COMAR)/UNEP Workshop with advanced training. Phuket Marine Biological Centre, Thailand, 10-26 February 1984. Unesco Reports in Marine Science 33.

73. Verheij, E. and Borel Best, M. 1987. Notes on the genus Polycyathus Duncan, 1876 and a description of three new scleractinian corals from the Indo-Pacific. Zool. Meded. 61, No. 12.

74. Veron, J.E.N. 1985. New scleractinia from Australian coral reefs. Rec. West. Aust. Mus, 12 (1): 147-183.

75. Veron, J.E.N. 1986. Corals of Australia and the Indo-Pacific. Angus and Robertson, North Ryde (N.S.W.).

76. Veron, J.E.N. 1990. Checklist of the hermatypic corals of Vanuatu. Pacific Science 44 (1): $51-70$.

77. Veron, J.E.N. 1990. Re-examination of the reef corals of Cocos (Keeling) Atoll. Rec. West. Aust. Mus. 14 (4): 553-581.

78. Vecon, J.E.N. 1990. New scleractinia from Japan and other Indo-West Pacific countries. Galarea 9: 95-173.

78a. Veron, J.E.N. 1992. Hermatypic corals of Japan. Aust. Inst. Mar. Sci. Monogr. 9: 1-234.

79. Veron, J.E.N. and Hodgson, G. 1989. Annotated checklist of the hermatypic corals of the Philippines. Pacific Science 43 (3): 234-287.

80. Veron, 3.E.N. and Marsh L.M. 1988. Hermatypic corala of Westem Australia: records and annotated species list. Rec. West. Aust. Mus. Suppl. 29: 1-136.
81. Vecon, J.E.N. and Pichon, M. 1976. Scleractinia of Eastem Australia. Part I. Families Thamnasteridae, Astrocoeniidae, Pocilloporidae. Aust. Inst. Mor. Sci. Monogr. Series 1., 86pp.

82. Veron, J.E.N. and Pichon, M. 1980. Scleractinia of Eastern Australia. Part III. Families Agariciidae, Siderastreidac, Fungiidac, Oculinidac, Merulinidae, Mussidac, Pectiniidae, Caryophylliidae, Dendrophylliidae. Aust. Inst. Mar. Sci. Monogr. 4: 443pp.

83. Veron, J.E.N. and Pichon, M. 1982. Scleractinia of Eastern Australia. Part IV. Family Poritidae. Aust. Inst. Mar. Sci. Monogr. 5: 159pp.

84. Veron, J.E.N., Pichon, M. and Wijsman-Best, M. 1977. Scleractinia of Eastem Australia. Part II. Familiea Faviidae, Trachyphylliidae. Aust. Inst. Mar. Sci. Monogr. 3: J-233.

85. Veron, J.E.N. and Wallace, C. J984. Scleractinia of Eastern Australia. Part V. Family Acroporidae. Aust. Inst. Mar. Sci. Monogr. 6: 485 pp.

86. Wallace, C.C. 1978. The coral genus Acropora (Scleractinia: Astrocoeniina: Acroporidae) in the central and southem Great Barrier Reef Province. Mem. Qd. Mus. $18(2): 273-319$

86a. Weend, W.H. 1984. Taxonomic charactera in Caribbean Millepora species (Hydrozoa, Coelenterata). Bijdr. Dierk. 54: 243-262.

87. Wells, J.W. 1950. Reef corals from Cocos-Keeling Atoll. Bull. Raffles Mus. 22: 29-52.

88. Wells, J.W. 1954. Recent corals of the Marshall Islands. U.S. Geal. Surv. Prof. Pop. 260-I: 385-486.

89. Wells, J.W. J966. Evolutionary development in the scleractinian family Fungiidae. In W.J. Rees (ed): The Cnidaria and their evolution. Symp. Zool. Soc. Lond. 16: 223-246.

90. Wells, J.W. 1972. Notes on Indo-Pacific s-leractinian corals, VIII. Scleractinian corals from Easter Island. Pac. Sci. 26 (2): $183-190$

9]. Wells, J.W. 1973. Two new hermatypic scleractinian corals from the West Indies. Bull. Mar. Sci. 23 (4): 925-932.

92. Wells, J.W. 1982. Notes on Indo-Pacific corals. Part 9. New corals frem the Galapagos Islands. Pac. Sci. 36: 211-219.

93. Wells, J.W. 1983. Annotaled list of the scleractinian corals of the Galapagos Islands. In P.W. Glynn and G.M. Wellington (eds.): Corals and coral reefs of the Galapogas islands, p. 211-295. University of California Press, Berkeley.

94. Wells, J.W. 1986. A list of scleractinian generic and subgeneric taxa, J758-1985. Fossil Cnidaria 15(11): 1-69.

95. Wells, J.W. and Davies, P.S. 1966. Reef sludies at Addu Atoll. IV Preliminary list of stony corals from Addu Atoll. Atoll Res. Bull. No 116: 43-55.

96. Wells, J.W. and Lang, J.C. J973. Appendix: Systematic list of Jamaican shallow-water Scleractinia. Bull. Mar. Sci. 23 (J): $55-58$.

97. Wijsman-Best M. 1972. Systematics and ecology of New Caledonian Faviinae (Coelenterata- Scleractinia). Academisch Proefschrif. Bijdragen tot de Dierkunde 42 (J). 
98. Wijsman-Best M. 1974. Biological results of the Snellius Expedition. XXXV. Faviidac collected by the Snellius Expedition. 1. The genus Favia. Zool. Meded. 48(22) 249-261.

99. Wijsman-Beat M., Faure, G. and Pichon, M. 1980. Contribution to the knowledge of the stony corals from the Seychelles and Eastern Africa. Rev. Zool. ofr. 94(3): $600-627$.

100. Wood, E.M. 1983. Corals of the world. T.F.H. Publications, Neptune City (N.J.).

101. Wood, E.M. and Tan B.S. 1987. The coral reefs of the Bodgaya Islands (Sabah: Malaysia) and Pulau Sipadan 3. Hard corals. Malay. Nat. J. 40: 189-224.

102. Woodhead, P.M.J. and Weber, J.N. 1969. Coral genera of New Caledonia. Mar. Biol. 4(3): 250-254.

103. Yabe, H. and Eguchi, M. 1932. A study of the recent deep water coral fauna of Japan. Proc. imp. Acad. Japan 8: 387-390.

104. Zibrowius, H. 1973. Revision des espèces actuelles de genre Enallopsammia Michellotti, 1871, et description de D. marenzelleri, nouvelle espèce bathyale à large distribution: Océan Indien et Aclantique Central (Madreporaria, Dendrophylliidae). Beaufortia 21, No. 276.

105. Zibrowius, H. 1974. Scleractiniares des iles Saint Paul et Amsterdam (sud de l'océan Indien). Tethys 5 (4); 747-777.
106. Zibrowius, H. 1974. Révision du genre Javania et considérations générales sur lea Flabellidae (Scléractiniaires). Bull. Inst. océanogr. Monaco 71, No. 1429: $48 \mathrm{pp}$.

107. Zibrowius, H. 1980. Les Scléractiniaires de la Méditerranée et de l'Atlantique nord-oriental. Memoires de l'Insnitul Oceanographic Manaco No. 11.

108. Zibrowius H. and Cairns, S.D. 1992. Revision of the northeast Allantic and Mediterranean Stylasteridae (Cnidaria: Hydrozoa). Mémoires du Muséum Narional d'Histoire Naturelle, Paris Zoologie. Tome (A) 153.

109. Zibrowius, H. and Gili, J.M. 1990. Deep-water Scleractinia (Cnidaria: Anthozos) from Namibia, South Africa and Walvis Ridge, Southeastern Allantic. Scient. Mar. 54(1): 19-46.

110. Zibrowiua, H. and Grieshaber, A. 1977. Scléractinaires de l'Adriatique. Tethys 4: 375-384

111. Zou Renling, Song S.W. and Ma J.H. 1975. [Reef-building corals of shallow waters of Hainan Island.] Peking Science Press. (In Chinese except for scientific names).

112. Zou Renling, Meng Z. and Guan X. 1983. [Ecological analyses of hermatypic corals from the northern shelf of the South China Sea.J Tropic Oceanology 2(3): 1-6. (In Chinese with English abstract). 


\section{INDEX AND SYNONYMY}

Square brackets indicate hard coral reference numbers (see pages 137-140).

abbreviata, Achatinella 21

abdita, Favites 106

abies, Antipaches 35

abies, Gorgonia Linnaeus 1758 = Antiparhes abies 35

abies var. paniculaza, Antipathes $=$ A. paniculata 38

abietina, Antipathes Pourtalés 1874

= Aphanipathes abietina 41

abietina, Aphanipathes 41

abietina, Parantipathes (Pourtalès 1874)

= Aphanipathes abietina 41

abrolhosensis, Acrapara 65

abrotanoides, Acropora (Lamarck 1816) = A. danai [65] 66

abrotanaides, Mantipara (Audouin)

$=M$. circumvallata [65] 70

abrupta, Lampsilis 19

abyssicola, Stichopathes 45

abyssanum, Caryaphyllia 114

Acanihasirea 95

Acanthelia Wells 1937 = Echinopora 104

Acanthacyathus Milne Edwards \& Haime 1848 = Caryophyllia 114

Acanthophyllia 95

Acanthophyllia Wells $1937=$ Cymarina [2] 96

Acanthopora Verrill 1864 = Echinopara 104

acervata, Acropora (Dana 1846) = ?A. humilis 66

acervala, Acropora 65

Achatinella 21-23

Acipenser 1

Acrhelia 90

acrolophos, Lepidapora 56

Acropora 65

Actinastrea 61

actiniformis, Fungia Quoy \& Gaimard 1833

= Heliafungia actinifarmis 86

actiniformis, Heliafungia 86

aculeata, Antipathes 35

aculeata, Arachnopathes Brook 1889

$=$ Antipathes aculeata 35

aculeatum, Flabellum Milne Edwards \& Haime 1848

= Truncatoflabellum aculeatum [23] 129

aculeatum, Truncatoflabellum 129

aculeus, Acropora 65

acuminata, Acropora 65

acuta, Pacillopara Lamarck $=P$. damicomis $[65] 63$

acutata, Montipara 70

acuticarinaza, Pavana 81

acuticallis, Faviles (Ortmann 1889) $=F$. chinensis [65] 106 acutidens, Fungia Studer $1877=F$. horrida [44] 85

Adelapara 52

adeva, Conopora 53

admiralitatus, Omithoptera priamus 10

adrianae, Fungia van der Horst 1921

$$
=\text { Cycloseris cyclalites } 84
$$

aeacus, Traides 12

aeacus, Troides aeacus 12

aenea, Antipathes (Linnaeus 1758) $=A$. abies 35

aenea, Antipathes G. von Koch $1889=$ A. dichorama 36

aenea, Gargania Linnaeus $1758=$ Antipathes abies 35

aequicostatus, Heteracyathus 119

aequituberculata, Montipara 70

aesacus, Omithaptera 9

aesacus, Omithoptera priamus $=O$. aesacus 9

affine, Flabellum Milne Edwards \& Haime 1848

= Truncatoflabellum spheniscus [23] 129 affinis, Balanophyllia 131

affinis, Bathypathes (Brook 1889) = Schizopathes affinis 44

affinis, Crypthelia 53

affinis, Favia 105

affinis, Lophahelia Pourralès 1868

= Lophelia pertusa [107] 120

affinis, Rhizatrachus Duncan 1873

$=$ Monomyces pygmaea [107] 128

affinis, Schizapathes 44

africana, Acropora (Brook 1893) = A. tenuis [65] 68

africana, Gyrapora 55

agaricia, Symphyllia 99

Agaricia 79

Agariciella Ma 1937 = Gardineroseris 79

agaricifarmis, Fungia Lamarck $=F$. fungites [65] 85

agariciles, Agaricia 79

agassizi, Cyphastrea 103

agassizii, Deltocyathus 117

Agelecyathus Duncan 1876 = Polycyathus 90, 123

aggregata, Cirrhipathes 42

aggregata, Stichopathes van Pesch 1914

= Cirrhipathes aggregata 42

aithoseptatus, Trachocyathus 126

akajimensis, Acrapara 65

akakeae, Omithoptera 9

alabastrum, Desmophyllum Alcock 1902

$$
\text { = Placatrochides alabastrum [107] } 128
$$

alabastrum, Flabellum 126

alabastrum, Placotrochides 128

alaskana, Caryaphyllia 114

alaskanus, Srylasier 58

alata, Antipathes 35

alata, Aphanipathes Brook $1889=$ Antipathes alata 35

alberti, Caryophyllia 114

albescens, Traganoptera broakiana 12

albitentaculata, Ctenactis 83

albitentaculata, Crenacris Hoeksema 1989

$$
=\text { ?C. echinata }[78 \mathrm{~g}] 83
$$

alcalai, Alveopara $=$ A. spangiosa 72

alces, Acropara 65

alcicamis, Millepora 51

alcicamis, Millepora Linnaeus $1758=?$ M. tenera $[50] 51$ alcicarnis, Pecrinia 94

alcacki, Caryophyllia Vaughan $1907=$ C. atlantica 115

alcacki, Dendrophyllia 132

alcocki, Srichopathes 45

aleuticus, Fungiacyathus 83

aleuticus, Fungiacyathus Keller 1976

$$
=? F \text {. marenzelleri }[? 23] 83
$$

alexandrae, Aetheoptera $=$ Omithaptera alexandrae 9 alexandrae, Omithaptera 9

aliciae, Mycetaphyllia Wells $1973=?$ M. Lamarckiana 98

aliciae, Mycetophyllia 98

allingi, Alveopora 72

allonei, Aetheoptera = Omithaptera allottei 9

allottei, Omithoptera 9

alnutti, Distichopora Wright $1882=D$. nitida $[14] 54$

alta, Fungia Nemenzo 1983

$$
=F \text {. gravis [44], =F. paumatensis [79] } 85
$$

alta, Galaxea 91

alsa, Oulastrea Nemenzo $=0$. crispa [79] 110

aliasepia, Mantipora 70

altemans, Trematotrachus 125

altemata. Bathypathes 41 
altemata, Dendrophyllia 132

alsematus, Heterocyathus 119

alrispina, Errina 54

alrispina, Lepidotheca 56

alveolata, Porites Milne Edwards \& Haime $=P$. solida $[65] 75$

alveolus, Meandrina 113

alveolus, Placotrochus Duncan 1863

= Meandrina alveolus [23] 113

alveopora, Montipora 70

Alveopora 72

amakusensis, Acanthastrea 95

amaranthus, Colpophyllia 103

ambrosia, Caryophyllia 114

americana, Antipathes 35

americana, Phyllangia 90

amicorum, Barabanoia 101

amicorum, Favia (Milne Edwards \& Haime 1850)

= Barabanoia amicorum 101

amitoriensis, Leptoseris 80

amoena, Errina Boschms $1956=E$. dabneyi [14] 54

Amphelia 90

amphelioides, Enallopsammia 133

amphelioides, Stylaster 58

amphrysus, Troides 12

amphrysus, Troides amphrysus 12

ampla, Herpetolitha Verrill $1864=$ Herpolitha limax [44] 87

ampliata, Merulina 100

Anacropora 68

anceps, Distichopora (Haplomerismos) 54

ancora, Euphyllia 119

andamanensis, Polycyathus 123

andamanicus, Deluocyathus 117

andrewei, Traides amphrysus 12

andrewianus, Sphenotrochus 124

andrewsi, Porites Vaughan $1918=P$. cylindrica [65] 74

andromache, Troides 12

andromache, Troides andromache 12

Angia Milne Edwards \& Haime 1848 = Culicia 89

angiostomum, Flabellum 126

anguina, Antipathes Dans 1846 = Cirrhipathes anguina 42

anguina, Cirrhipaches 42

angulare, Flabellum 126

angulata, Acropora 65

angulata, Montipora 70

angulata, Seriatopora Klunzinger $=S$. hysirix [65] 64

angulosa, Acanthastrea ? = A. bowerbanki [65] 95

angulosa, Mussa 98

angusia, Monnipora $=$ M. friabilis [79] 70

Anisopsammia Marenzeller 1904 = Enallopsammia 133

ankeli, Pocillopora 63

annae, Porites 74

annectens, Paryphanta 23

annectens, Powelliphanta = Paryphanta annectens 23

annularis, Montastrea 109

annulata, Guynia 130

annuligera, Montastrea 109

anomala, Distichopara 54

Anomastraea 76

Anomocora 114

antarctica, Caryophyllia 114

antarctica, Errina 54

antarctica, Gardineria 128

antarctica, Javania 128

antarcticus, Leptopenus 82

Anthemiphyllia 113

anthocencis, Acropora (Brook 1893) = ?A. eurystoma [64] 66

anthocercis, Acropora 65

anthohelia, Conopora 53 anthophyllites, Coenocyathus 116

anthophyllites, Pouralosmilia 124

anthophyllum, Biflabellum (Ehrenberg 1834)

= Monomyces pygmaea [107] 128

anthophyllum, Flabellum (Ehrenberg 1834)

= Monamyces pygmaea [107] 128

anthophyllum, Monamyces 128

anthophyllum, Monamyces Ehrenberg 1834

$=$ M. pygmaea [107] 128

antileuca, Troides helena 13

antillarum, Coryophyllia 114

antillarim, Stylaster 58

antiope, Troides hypolitus 13

Antipathella Brook $1889=$ Antipathes 35

Antipathes 35

aotearoa, Flabellum 126

aperta, Antipathes 35

aperta, Monsastrea 109

apertes, Coencyathus Dōdarlein 1913

= Phyllengia mouchezii [107] 90

aperium, Flabellum 127

apexfulva, Achatinella 21

Aphanipathes 41

Aphrastrea Milne Edwards \& Haime 1848 = Favites 106

aphrodes, Heteropsammia Alcock $=$ H. cochlea [65] 133

Aplocyathus d'Orbigny $1849=$ Trochocyathus 126

apoensis, Troides magellanus 13

apollo, Pamassius 11

Apollo 11

appressa, Acropora 65

Arachnopathes Milne, Edwards \& Haime 1857

= Antipathes 35

Araeacis Milne Edwards \& Haime 1849 = Astreopora 69

aranetai, Porites 74

Arapaima 2

Arapaima 2

arborea, Antipathes 35

arbuscula, Acropora (Dans 1846) = A. formosa 66

arbuscula, Acropora 65

arbuscula, Cladocora 102

arbuscula, Coenosmilia Pourtalès 1874

= Anomocora fecunda [107] 114

arbuscula, Dendrophyllia 132

arbuscula, Lophelia Moseley 1881

= Madrepora arbuscula [10] 92

arbuscula, Madrepora 92

arbuscula, Oculina 92

archeri, Omithoptera victoriae 11

Archohelia 91

arcticans, Ulocyathus Sars 1851

= Flabellum macandrewi [107] 127

arcuata, Acropora (Brook) = A. cytherea [65] 66

arcuala, Caryophyllia 115

arcuatus, Paracyathus 122

arenosa, Porites $($ Esper 1797) $=$ P. lutea [65] 75

areolata, Manicina 108

areum, Flabellum 127

arfakensis, Omithoptera paradisea 10

ariadne, Troides haliphron 12

armala, Favia 105

armata, Stylocoeniella 61

amoldi, Caryophyllia 115

Arowana, Asian 2

arruana, Omithoptera priamus 10

asanoi, Madracis 62

ashmorensis, Echinopora 105

asper, Stylaster 58

aspera, Acropora 65

aspera, Echinophyllia 93 
aspera, Emina 54

aspera, Goniastrea (Verrill 1865) = G. favulus [97] 107

aspera, Montipora 70

aspera, Oulophyllia (Quelch 1886) = o. crispa [65] 110

aspera, Pocillopora 63

asperata, Fungia Dana $1846=$ Cienactis echinata [44] 83

asperula, Madracis 62

aspidopora, Asrya 52

assimilis, Antipathella Brook $1889=$ Antipathes assimilis 35 assimilis, Antipathes 35

Asteroseris Verrill 1901 = Pavona 81

Asterosmilia 114

astraeiformis, Astrangia Milne Edwands \& Haime 1850

$$
\text { = A. poculata } 89
$$

Astrangia 89

Astrea Lamarck 1801 = Siderastrea/Favia 78/105

astreata, Galarea 91

astreiformis, Platygyra (Mitne Edwards \& Haime 1849)

$$
=P \text {. doedalea }[65,97] 111
$$

astreoides, Porites 74

Astreoides Blainville $1830=$ Astroides 130

Astreopora 69

Astreosmilia 101

Astroides 130

Astrophyllia Ehremberg $1834=$ ?Favites 106

Astropsammia 130

Astropsammia Verrill $1869=$ ?Tubastraea [25] 135

Astroria Milne Edwards \& Haime $1848=$ Platygyra 110

Astya 52

allantica, Antipathello (Gray 1857) = Antipathes allantica 35

allantica, Antipathes 35

atlantica, Caryophyllia 115

allantica, Errina 54

atlantica, Paratylopathes Roule 1902 = Antipathes grayi 37

allanicum, Flabellum 127

atlanticus, Aulocyathus 114

atlanticus, Polycyathus 123

allanticus, Stylaster 58

atrata, Dendrophyllia 132

atrenuata, Acropora 65

artenuala, Porites 74

attenuata, Turbinaria Nemenzo $=T$, irregularis [79] 136

Aulocyathus 114

aurantiacus, Sphenotrochus 124

aurantiacus, Stylaster $\mathbf{5 8}$

aurea, Tubastraea 135

auritus, Sphenotrochus 124

austera, Acropora 65

australe, Flabellum 127

australensis, Goniastrea 107

australiae, Endopachys 133

australiae, Plesioseris 77

australiensis, Culicia 89

australiensis, Montipora 70

australiensis, Peponocyathus 122

australiensis, Porites 74

australis, Scolymia 99

Australocyachus 114

Australogyra 101

Australomussa 95

avis, Cyathoceras (Dutham \& Barnard 1952)

= Pseudocyathoceras avis [25] 124

avis, Pseudocyathoceras 124

Axhelia Milne Edwards \& Haime 1849 = Madracis 62

axifuga, Duncanopsammia 132

arillaris, Cyathelia 91

Axohelia Milne Edwards \& Haime $1857=$ Madracis 62

ayleni, Physophyllia 95

azurea, Acropora 65 baeodactyla, Acropora (Brook) = A. digitifera [65] 66

bairdi, Flagellum Milre Edwards \& Haime 1848

= Truncatoflabellum baindi [23] 129

bairdi, Truncaroflabellum 129

bairdiana, Balanophyllia 13!

balaenacea, Caryophyllia 115

Balanophyllia 131

balia, Crypthelia 53

bandensis, Troides oblangomaculatus 13

banksii, Oculina 92

Bantamia Yabe \& Eguchi 1943 = ?Galaxea 91

banyulensis, Polycyathus Best $1968=P$. muellerae [107] 124

Barabatroia 101

baracoensis, Porites 74

Barb Fish, African Blind 2

barbadensis, Antipathes 36

barbadensis, Aphanipathes Brook 1889

$=$ Antipathes barbadensis 36

barbadensis, Caryophyllia 115

barbadensis, Distichopora 54

bartschi, Coenocyathus Wells 1947

$=$ Rhizosmilia maculata 124

bartschi, Rhizosmilia (Wells 1947) = R. maculata 124

Banyastrea Milne Edwards \& Haime 1848 = Leptastrea 107

Bachelia 91

Bathyactis Moseley 1881 = Fungiacyathus [23] 83

Bathycyathus Milne Edwards \& Haime 1848 = Phyllangia 90

Bathypathes 41

Bathypsammia 131

Bathytrochus Gravier 1915 = Fungiacyathus [107] 83

Batotrochus Wells $1937=$ Trematotrochus 125

bayeri, Balanophyllia 131

bazilanicus, Troides $=T$. hadamantus 13

bellula, Acharinella 21

bellus. Stylaster 58

belzanor, Troides $=T$. rhadamantus 13

benhami, Goniastrea Vaughan 1917

$=$ G. australensis [97] 107

bennettae, Favites Veron, Picon \& Wijsman-Best

= Oulophyllia bennetlae 110

bennettae, Oulophyllia 110

bemardi, Goniopora 73

bemardi, Montipora 70

bemardi, Porites 74

berryi, Montipora 70

berryi, Montipora Hoffmeister $1925=M$. informis 71

berteriana, Caryophyltia 115

bewickensis, Lepiasirea 108

Bhutan Glory 9

Bhutanitis 9

bicolar, Errina 54

bifaria, Anripathes 36

bifida, Bathypathes 41

Biflabellum Döderlein $1913=$ Manomyces 128

biformis, Montipora Nemenzo = M. mollis [79] 71

bifrons, Turbinaria 136

bifuncala, Acropora Nemenzo = A. hyacinthus [79] 66

Bikiniastrea Wells 1954 = Barabattoia 101

bilaninata, Montipora 70

bilobatus, Stylaster 58

bipartila, Pavona 81

Birdwing, Black and Gold 13

Birdwing, Buru Opalescent 13

Birdwing, Chimaers 10

Birdwing, Common 10, 13

Birdwing, Common Green 10

Birdwing, D'Urville's 10

Birdwing, Golden 12 
Birdwing, Goliath 10

Birdwing, Malay 12

Birdwing, New Guinea 10

Birdwing, Paradise 10

Birdwing, Priam'a 10

Birdwing, Queen Alexandra's 9

Birdwing, Queen Victoria's 11

Birdwing, Rajah Brooke's 12

Birdwing, Richmond 10

Birdwing, Rothschild'a 10

Birdwing, Small 12

Birdwing, Tailed 10

Birdwing, Talaud Black 12

biserialis, Lepidopora 56

bispinosa, Stichopathes Summers $1910=$ S. flagellum 45

bichalamus, Stylaster 58

Blastomussa 95

Blastosmilia Duncan 1878 = Pourtalosmilia 124

Blastotrochus 126

blaneus, Stylaster 58

bocki, Stylaster 58

boisduvali, Omithoptera priamus 10

Boninastrea 99

boninensis, Boninastrea 99

bonsai, Hydnophora 100

Bonytongue, Asian 2

borchi, Omithaptera paradisea 10

borealis, Distichopora 54

boreopacificus, Stylaster 58

bomemanni, Ornithoptera priamus 10

borradailei, Orbicella Gardiner 1904 = Favia favus [97] 106

boschmai, Errina 54

boschmai, Millepora 51

boschmai, Stylaster 58

boscii, Anripathella (Lamouroux 1816) = Antipothes boscii 36

boscii, Anripothes 36

boscii, Leiopathes (Lamouroux ?) = Antipathes boscii 36

bottae, Coscinaraea 76

boltae, Leptastrea 108

bottae, Maeandroseris 77

boumei, Stichopathes 45

Boumeotrochus 114

boumoni, Solenastrea 111

bouruensis, Troides oblongomaculatus 13

bowerbanki, Acanthastrea 95

bowersi, Coenocyathus 116

brachiata, Acropora (Dana 1846) = A. nobilis 67

Brachymaeandrina Duncan $1884=$ Platygyra 110

Brachypelma 14

brachystoma, Fungia Thiel 1932 = Ctenactis crassa [44] 83

Brachytrochus Duncan $1876=$ ?Culicia 89

bradleyi, Oulangia 90

branneri, Porikes 74

brasseyae, Distichopora Wright $1882=D$. nirida [14] 54

Brasseyia Wright $1882=$ Dendrophyllia 132

brassica, Turbinaria 136

braziliensis, Astrangia Vaughan $1906=$ A. solitaria 67

braziliensis, Meandrina (Milne Edwards \& Haime 1849) $=$ M. meandrites 113

braziliensis, Millepora 51

braziliensis, Mussismilia 98

brevicollis, Acropora (Brook) = A. digirifera [65] 66

breviconus, Hydnophora Nemenzo $=H$. rigida [79] 100

brevicomis, Pocillopora Lamarck 1816

$$
=P \text {. damicomis [65] } 63
$$

brevicula, Lampsilis 19

brevicula, Lampsilis reeviana $=$ L. brevicula 19

brevicula, Ligumia reeviana = Lampsilis brevicula 19

brevirostris, Acipenser $=$ A. brevirostrum । brevirostrum, Acipenser 1

breviserialis, Colpophyllia 103

breviserialis, Distichopora Quelch $1884=$ D. nitida [14] 54

brighami, Parikes 74

brighami, Psammocora 77

britannica, Leptopsammia 134

brochi, Lepidotheca 56

brochi, Stylaster 58

brooki, Antipachella Johnson 1899 = Antipathes atlantica 35

brookiana, Omithoptera = Trogonoptera brookiana 12

brookiana, Trogonoptera 12

brookiana, Trogonoptera brookiana 12

brookiana, Troides = Trogonoptera brookiana 12

browni, Astrangia 89

brueggemanni, Acropora 65

brueggemanni, Madracis 62

brueggemanni, Montipora Bernard $=M$. danae [65] 70

brunneus, Conotrochus 116

brunneus, Stylaster 58

buccina, Balanophyllia 131

buddii, Achatinella 21

bulbosa, Pocillopora Ehrenberg $1834=P$. damicomis [65] 63

bulimoides, Acharinella 21

burchae, Premocyathus 124

burgosi, Goniopora 73

burkei, Omithoptera priamus $=0$. urvillianus 10

busbyi, Paryphanta 23

bushyensis, Acropora 65

Butterfly of Paradise 10

byronii, Acharinella 21

cacharias, Fungia Studer $1877=F$. paumotensis [44] 85 cactus, Montipora 70

cactus, Mussa 98

cactus. Pavona 81

Caccobarbus 2

caelata, Conradilla 17

caelestis, Omithoptera

caelestis, Omithoptera priamus = C. caelestis 9

caesia, Achatinella 21

caespitosa, Cladocora 102

cailleri, Agaricia (Duchassaing \& Michelotti 1864)

$$
\text { = Leptoseris cailleri [35] } 80
$$

cailleti, Javania 128

cailleti, Leptoseris 80

cailleti, Mycedium Duchassaing \& Michelotti 1864

$=$ Leptoseris cailleti [35] 80

calamaria, Acropora (Brook) = A. humilis [65] 66

calcar. Deltocyathus 118

calcarea, Montipora 70

calicifera, Pavona 81

caliculata, Montipora 70

caliendrum, Seriatopora 64

califomica, Dendrophyllia 132

californica, Nomlandia 121

californicus, Stylaster 58

Callogyra 112

Callogyra Verrill 1902 = Trachyphyllia [57a] 112

calthus, Paracyathus 122

calveri, Coryophyllia 115

calycularis, Astroides (Pallas 1766) = ?Acropora luteus 67

calycularis, Astroides 130

Calyptopora 52

campaniformis, Stephanoryathus 125

campanulatum, Flabellum 127

campylecus, Stylaster 58

canalis, Acropora (Quelch) = ?A. nobilis 67

canalis, Acropora 65

cancellata, Aphanipathes 41 
cancellotus, Stylaster $\mathbf{5 8}$

candeanum, Flabellum Milne Edwards \& Haime 1848 = Truncatoflabellum candeanum [23] 129

candeanum, Truncatoflabellum 129

candearus, Placotrochus Milne Edwards \& Haime 1848 $=P$. Laevis [23] 128

candelabrum, Conopora 53

candida, Bathelia 91

candida, Lophelia Moseley 1881

= Madrepora candida [107] 92

candida, Madrepora 92

Cantharellus 83

Cantharellus Hoeksema \& Best $1984=$ Cycloseris 84

capax, Potamilus 20

capax, Proptera $=$ Potamilus capax 20

capensis, Coryophyllia 115

capensis, Errina 54

capensis, Gandineria 128

capillaris, Acropora 65

capitata, Montipora 70

capilata, Pocillopora 63

capricomis, Montipora 70

carcarensis, Turbinaria Nemenzo $=T$. frondens [79] 136

candenae, Acropora 65

carduus, Acropora 65

carduus, Echinopora KLunzinger = E. gemmacea [65] 105

caribbeana, Balanophyllia 131

carinata, Anomocora 114

carinata, Errina (Pourtalès 1867)

= Lepidopora carinata [18] 56

carinata, Heliopora Pourtalès 1867

= Lepidopora carinala [18] 56

carinata, Lepidopora 56

carinata, Montipora Nemenzo = M. hirsula 71

carinata, Pachyseris 81

carinata, Turbinaria Nemenzo $=T$. stellulata [79] 136

carinatum, Truncatoflabellum 129

carinatus, Stylaster $\mathbf{5 8}$

carli, Plesiastrea Nemenzo = Favia stelligera [79] 106

camea, Errina Boschma $1964=E$. laterorifa [14] 55

carolina, Lophelia Pourtalès 1871

= Madrepora carolina [107] 92

carolina, Madrepora 92

carolinensis, Cryptotrochus 117

caroliniana, Acropora 65

carpenteri, Caryophyllia Duncan 1878

= Stenocyathus vermiformis [107] 130

Carunculina 20

Caryaphyllia 114

casta, Achatinella 21

Cat, Duckbill 1

Cat, Spoonbilt 1

calalai, Alveopora 72

Catalaphyllia 115

Catfish, Giant 2

catharinae, Antipathes 36

catharinae, Aphanipathes (Pax 1932)

= Antipathes catharinae 36

Caulastrea 102

caulifera, Balanophyllia 131

cavemosa, Favia 106

cavemosa, Madrepora Forskà] 1775 = Favia favus [97] 106

cavemosa, Montastrea 109

cebuensis, Montipora 70

celebensis, Troides criton 12

cellularis, Troides hypolitus 13

cellulosa, Balanophyllia 131

cellulosa, Favia (Verrill 1872) = F. pallida 106

cellulosa, Goniopora 73 cellulosa, Stylophora Quelch $=S$. pistillata [79] 64

Ceratocyathus Seguenza 1863 = Caryophyllia 114

Cerasosrochus 116

cerberus, Troides helena 13

cerealis, Acropora 66

cerebriformis, Mussa 98

Ceriomorpha Head $1978=$ Blastomussa 95

cerium, Goniastrea (Dana 1846) = G. pectinata 107

cervicomis, Acropora 66

cervicomis, Dactylotrochus 117

cervicomis, Lepidotheca 56

cervina, Distichopora 54

cespitosa, Pocillopora Dana $3846=P$. damicomis [65] 63

cestoporina, Eminopora 55

cestus, Achatinella 21

ceylonensis, Antipathella (Thomson \& Simpson 1905)

$$
=\text { Antipathes ceylonensis } 36
$$

ceylonensis, Antipathes 36

ceylonensis, Stichopathes 45

ceylonica, Acropora 66

chagius, Cienella 112

chalcidicum, Cyphastrea 103

challengeri, Stenohelia Boschma $1951=S$. profinda [14] 57

challengeri, Stylaster Boschma 1957

$$
\text { = Stenohelia profiunda [18] } 57
$$

chalumnae, Latimeria 1

chamaemorus, Antipaches 36

chamissoi, Rhizopsammia 134

charybdis, Omithoptera chimaera 10

Chasmistes 2

chathamensis, Errina 54

chauliostylus, Lepidosheca 56

cheilopora, Errina 54

Cheiloporidion 52

chesterfieldensis, Acropora 66

chevalier, Leptopsammia 134

chikae, Papilio 11

chimaera, Omichoptera 10

chimaera, Omichoptera chimaera 10

chimaera, Phalaenosoma = Omithoptera chimaera io

chimaera, Schoentergia = Omithoptera chimaera 10

chimaera, Traides = Omithoptera chimaera 10

chinersis, Faviles 106

chnous, Balanophyllia 131

chota, Antipathes 36

chrysanthemum, Omithoptera paradisea 10

chunii, Flabellum 127

cicatricosus, Plethobasus 19

cinnabarina, Distichopora Nardo $1844=D$. violacea [] 4$] 54$

circumvallata, Montipora 70

Cirrhipathes 42

Citharocyathus Alcock 1902 - Notocyathus [23] 121

Cladangia 89

Cladocora 102

Cladopathes 43

Cladopsammia 132

Cladorbicella 102

Clam, Bear Paw 16

Clam, Boring 16

Clam, China 16

Clam, Crocus 16

Clam, Fluted 17

Clam, Giant 16

Clam, Horse's Hoof 16

Clam, Saffron-coloured 16

Clam, Scaly 17

Clam, Small Giant , 29

Clam, Southern Giant 16

Clam, Sirawberry 36 
Clam, Tevoro 17

clathra, Paracyathus 122

clachrata, Acroporo 66

clausa, Crypthelia 53

clava, Pleurobema 20

clavaria. Pocillapora 63

clavaria, Porites 74

Clavarina Verrill 1863 = Paraclavarina 100

clavator, Halamitra 86

clavigera, Lepidopora 56

clavus, Caryophyllia 115

clavus, Golarea (Dana 1846) = G. astreata [65] 91

clavus, Galaxea 91

clavus, Pavona 81

clementei, Pachyseris Nemenzo $=$ P. speciosa [79] 81

clivosa, Diploria 104

clivosa, Pavona 81

clypeus, Halamitra Verrill $1864=H$. pileus [44] 86 coalita, Montipora Nemenzo $=M$. aliasepta [79] 70

coalitum, Flabellum 127

coarctala, Dendrophyllia 132

coccinea, Distichopora 54

coccinea, Tubastraea 135

cochlea, Heleropsammia 133

cochleata, Errina 54

cochleato, Lepidopora (Pournalès 1867)

= Emina cochleala [18] 54

cocosensis, Montipora 70

cocosensis, Montipora Vaughan $1918=M$. angulata 70 cocosensis, Porites 74

cocosensis, Porites Wells $1950=$ ?P. cylindrico 74

cocosensis, Stylocoeniella 61

Coelacanth 1

Coelastrea 102

Coelocyathus Sars $1856=$ Monomyces 128

Coeloria Milne Edwards \& Haime 1848 = Platygyra 110

Coeloseris 79

Coelosmilia Milne Edwards \& Haime 1848

$$
=\text { Desmophyllum } 118
$$

Coenangia 89

Coenocyathus 116

Coenopsammia 132

Coenosmilio 116

coerulea, Heliopora 60

Colangia 89

colei, Montipora Wells $1954=M$, undata 71

colonensis, Porites 74

Colpophyllia 103

columna, Coscinaraea 76

columna, Goniopora 73

columna, Goniopora Dana $1846=$ ?G. planulata [65] 73

columna, Leptoseris Yabe \& Sugiyama 1941

$$
=\text { L. scabra }[35,65] 80
$$

columnaris, Antipathes 36

columnaris, Arachnopaches Duchassaing 1870

= Antipathes columnaris 36

columnaris, Montipora 70

columnaris, Parantipaihes (Duchassaing 1870)

= Antipathes columnaris 36

columnaris, Porites 74

compacta, Rhizopsammia 134

complanata, Acropora (Brook)

=A. clathrata/divaricata $[65] 66$

complanata, Calyptopora (Pourtalés 1867)

= Stylaster complanaus $[18] 58$

complanata, Favites 106

complanata, Millepora 51

complanato, Montipora 70

complanota. Pavona 81 complanatus, Stylaster 58

complicala, Stephanophyllia 82

composita, Montipora Crosaland 1952

$=M$. aequituberculata $[65] 70$

compressa, Porites 74

compressa, Stylophora 64

compressum, Truncatoflabellum 129

compressus, Platytrochus 123

compressus. Premocyathus 124

concavospira, Achatinella 21

concentrica, Halamitra Studer $1901=H$. pileus $[44] 86$

Concentrotheca 116

conceprus, Paracyathus 122

conceptus, Polycyathus 123

concinna, Acropora (Brook) = A. valida [65] 68

concinna, Echinopora Vaughan = E. lamellasa [65] 105

concinna, Fungia 85

concinna, Stenohelia 57

concinna, Vaughanella 126

confera, Acropora (Brook) = A. hyacinthus [65] 66

conferta, Coenangia 89

conferra, Cyphastrea Nemenzo = C. serailia [79] 103

conferia, Distichopora Quelch $1885=$ D. gracilis [14] 54

conferta, Lobactis Verrill 1864 = Fungia scutaria 86

conferia, Montipora Nemenzo 1967

$=$ M. monasteriata [79] 71

conferta, Pouralosmilia 124

conferra, Schizopathes 44

conferta, Stenohelia 57

confertifolia, Fungia Dana $1846=F$. fungises [44] 85

confiusa, Montipora 70

Congregopora 52

conicula, Montipora Wells $1954=$ M. millepora 71

conicus, Citharocyathus Yabe \& Eguchi 1941

$=$ Notocyathus venustus [23] 121

conicus, Deliocyathus 118

conicus, Notocyashus 121

conigera, Acropora (Dana 1846) = A. robusia 67

conigera, Acropora 66

connata, Astraeosmilia 101

Conocyathus 116

Conocyathus Brook 1893 = Acropora 65

Conopora 53

conotrochoides, Aulocyathus 114

Conotrochus 116

Conradilla 17

consagensis, Phyllangia 90

conspicua, Montipora Nemenzo $=$ M. orientalis [79] 71

conspicua, Turbinaria 136

contignatio, Hydnophora (Forskàl 1775) $=$ H. exesa $[65] 100$

contigua, Psammocora 77

contorta, Antipathella Brook 1889 = Antipathes contora 36

contoria, Antipathes 36

contorta, Cirrhipathes 42

contorta, Distichopora 54

contorta, Eucimpathes? ? = Cimhipathes contorta 42

contorza, Montipora Nemenzo \& Montecillo 1981

$=M$. confusa [79] 70

contona, Platygyra 111

contorta, Stichopathes 45

contorta, Stylophora 64

conuis, Flabellum 127

convera, Acropora (Dana 1846) = ?A. millepora 67

convera, Acropora 66

convexa, Poriles 74

convexa, Porites Verrill $=P$. nus [65] 75

cooperi, Cycloseris (Gardiner 1909) = C. tenuis 84

cooperi, Fungia Gardiner $1909=$ Cycloseris tenuis [44] 84

copiosa, Acropora 66 
cor, Quadrula 19

carallium, Stylaster 58

corbettensis, Montipara 70

carbicula, Batotrochus (Pourtalès 1878)

$=$ Tremalotrochus carbicula 125

corbicula, Tremalotrochus 125

carbicula, Truncatoflabellum 129

comigera, Dendrophyllia 132

comu, Balanophyllia 131

comu, Crispatotrochus (Moseley 1881)

= Cyazhoceras camu 117

comu, Cyathoceras 117

comu, Labyrinthocyathus 120

comucopia, Dendrophyllia 132

camufarmis, Caryophyllia 115

corona, Fungia 85

carona, Furgia Dōderlein $1901=$ ?F. scruposa [44] 86

caranata, Montastrea (Dana 1846) $=$ M. curta 109

coronatus, Odontocyathus 121

coronatus, Stephanocyathus 125

carrugata, Caryophyllia 115

corsicus, Coenocyathus Milne Edwards \& Haime

= Pouralosmilia anthophyllites [107] 124

corymbosa, Acropara 66

canymbosa, Lobophyllia 97

Coscinaraea 76

Coscinastrea Milne Edwards \& Haime 1848

$$
\text { = Cascinaraea } 77
$$

Cosmoporites Duchassaing \& Michelotti $1860=$ Porites 73 costata, Lobophyllia (Dana 1846) = L. hemprichii [65] 97

castata, Lobophyllia 97

castulata, Cyclaseris 84

castulata, Fungia Ortmann 1889 = Cycloseris costulata 84 crassa, Acropora 66

crassa, Coscinaraea 76

crassa, Ctenactis 83

crassa, Fungia Dans 1846 = Chenactis crassa [44] 83

crassa, Pavana (Dana) = P. decussata [65] 81

crassa, Schizopathes 44

crassa, Seriatopora 64

crassa, Seriatopora Quelch = ?S. hystrix 64

crassa, Turbinaria Bernard = T. mesenterina [79] 136

crassa, Turbinaria Nemenzo $=T$. mesenterina $[79] \quad 136$

crassilabrum, Adelopora 52

crassiar, Stylaster 58

crassispinosa, Oxypora 94

crassitentaculata, Fungia Quoy \& Gaimard 1833

= Heliafungia actinifarmis [44] 86

crassituberculata, Mantipora 70

crassolamellata, Fungia Milne Edwards \& Haime 1851

$=F$. fungives $[44] 85$

crassum, Flabellum Milne Edwards \& Haime 1848

= Truncatoflabellum crassum [23] 129

crassum, Truncatoflabellum 129

crassus, Herpetolithus Dans 1846 = Herpolitha limax [44] 87

crassus, Stephanocyathus 125

crater, Turbinaria 136

Craterastrea 77

crateriformis, Acropora 66

craterifarmis, Rhizotrachus Alcock $1893=R$. typus [23] 129

crenulatum, Flabellum Milne Edwards \& Haime 1848

= Truncatoflabellum profiundum [23] 129

crenulatus, Holcotrochus 120

cribripara, Acropora (Dana 1846) = A. aspera 65

cribripara, Parikes 74

crispa, Antipathes 36

crispa, Diaseris Pourtalè 1871

= Fungiacyathus crispus [107] 83 crispa, Haloseris Milne Edwards \& Haime 1851 $=$ Leploseris papyracea [35] 80

crispa, Merulina Dana $1846=$ M. ampliaca 100 crispa, Oulophyllia 110

crispa, Tylapathes Brook 1889 = Antipathes crispa 36 crispata, Oulastrea 110

Crispatotrochus 117

crispus, Fungiacyathus 83

cristagalli, Desmophyllum 118

cristagalli, Montipara 70

cristata, Euphyllia 119

criton, Troides 12

crilon, Troides crilon 12

crocea, Tridacna 16

croesus, Omithoptera 10

croesus, Omithoptera craesus 10

croesus, Omithoptera priamus $=0$. croesus 10

croesus, Priamoptera $=$ Omithoptera croesus 10

croesus, Priamusptera = Omithoptera croesus 10

crosslandi, Platygyra (Matthai 1928) = ?P. pini [65] 111

crasslandi, Plarygyra 111

cruenta, Errina 54

crustacea, Madrepora Pallas 1766

= Podabacia crustacea [44] 87

crustacea, Podabacia 87

cruzi, Millepora 51

Cryptabacia Milne Edwards \& Haime 1849 = Polyphyllia [44] 88

Crypthelia 53

cryprocymas, Lepidopora 56

cryptotrema, Crypthelia 53

Cryptotrachus 117

Clenactis 83

Ctenella 112

Ctenophyllia Dans 1846 = Meandrina 113

cubensis, Scalymia 99

cucullata, Astreopora 69

cucullata, Leptoseris 80

cucullata, Madrepora Ellis \& Solander 1786 $=$ Leptoseris cucullata [35] 80

Cui-ui 2

cujus, Chasmistes 2

Culicia 89

culiculata, Montipora 70

cultrifera, Caryophyllia 115

cumingii, Balanophyllia 131

cumingii, Flabellum Milne Edwards \& Haime 1848

= Truncatoflabellum cumingii [23] 129

cumingii, Truncatoflabellum 129

cumulaus, Parites 74

cuneala, Acropora 66

cuneifer, Traides 12

cuneifer, Troides cuneifer 12

cuneolus, Fuscanaia 19

cuneolus, Quadnula = Fusconaia cuneolus 19

cupressina. Antipathes Pallas $1766=$ A. abies 35

cupressus, Antipathes 36

curta, Achatinella 21

cura, Montastrea 109

curtisii, Dysnomia = Epiablasma curtisii 18

curtisi, Dysnomia forentina = Epioblasma curtisii 18

curtisi, Epioblasma 18

curtisii, Epiablasma florentina $=E$. currisii 18

curtisit, Plagiola $=$ Epioblasma currisit 18

curvata, Antipathes 36

curvata, Caulastrea 102

curvata, Crypthelia 53

curvata, Cyclaseris 84

curvata, Fungia Hoeksems 1989 = Cycloseris curvata 84 
curvatum, Flabellum 127

cuspidata, Acropora 66

cuticulata, Culicia 89

Cyathelia 91

Cyathina Ehrenberg 1834 = Caryophyllia 114

Cyathaceras 117

Cyathoceras Moseley 188 ] = ? Crispatotrochus [25] 117

Cyathohelia Milne Edwards \& Haime 1857 = Cyathelia 91

cyathoides, Balanophyllia 131

Cyathotrochus Boume 1905 = Tropidocyathus [23] 126

cyathus, Caryophyllia 115

Cyclicia Milne Edwards \& Haime 1851 = Culicia 89

Cyclohelia 54

cyclalites, Cycloseris 84

cyclolites, Fungia Lamarck $1801=$ Cycloseris cyclolites 84

cyclopea, Acropora (Dana 1846) $=$ ?A. rabusta 67

cyclopea, Acropora 66

cyclopora, Errinopora 55

Cycloseris 84

Cycloseris Milne Edwards \& Haime $1849=$ ?Fungia 85

Cyclosmilia d'Orbigny 1848 = Parasmilia 122

Cylicasmilia Milne Edwards \& Haime 1848 = Parasmilia 122

cylindr(ic)us, Dendragyra 112

cylindraceus, Tethocyathus 125

cylindrella, Canunculina = Toxolasma cylindrellus 20

cylindrellus, Toxolasma 20

cylindrica, Antipathes 36

cylindrica, Parites 74

cylindrica, Scapophyllia 101

cylindrica, Turbinaria Nemenzo $=T$. heronensis [79] 136

cylindricus, Coenocyathus 116

Cylindrophyllia 117

Cylloseris Quelch $1886=$ Leptaseris 80

cymas, Crypthelia 53

cymbicyathus, Acropara (Brook 1893) = A. nasura [50,65] 67

Cynarina 96

Cynoscion 3

Cyphastrea 103

cytherea, Acropora (Dana 1846) =?A. hyacinthus [64] 66 cytherea, Acropara 66

cytherea, Lobophyllia $($ Dana 1846) = L. hemprichii 97

dabneyi, Errina 54

dactylopama, Crypihelia 53

Dactylotrochus 117

daedalea, Alveopora 72

daedalea, Platygyra 111

damicamis, Pacillopara 63

danae, Astrangia Agassiz $1849=$ A. poculara 67

danae, Dendrophyllia 132

danae, Favia 106

danae, Favia Verrill $1872=F$. favus $[65,97] 106$

danae, Lobactis Verrill 1864 = Fungia scutaria [44] 86

danae, Mantipara 70

danae, Pacillopora Verrill $1864=P$. vernucosa [65] 63

danae, Porites 74

danae, Psammocora (Milne Edwards \& Haime 1851)

$$
=P \text {. contigua } 77
$$

danae, Stylaphara Milne Edwards \& Haime 1850 = S. pistillata [65] 64

danae, Turbinaria 136

Danafungia Wells 1966 = ?Fungia 85

danai, Acropora 66

danai, Favia 106

danai, Fungia (Milne Edwards \& Haime 1851) $=? F$. horridalscruposa [44] $85 / 86$

danai, Fungia 85

danai, Pavona 81 danai, Pavana Milne Edwards \& Haime 1860 $=? P$. cactus $[65] 81$

danai, Parites (Ellia \& Solander) = P. nus [65] 75

danana, Mycetophyllia 98

daniana, Mycetaphyllia Milne Edwards \& Haime 1849 $=$ ?M. Lamarckiana 98

daphnense, Flabellum 127

darsius, Troides 12

darsius, Troides helena $=T$, darsius 12

Dasmosmilia 117

Dasyphyllia Milne Edwards \& Haime 1848 = Caulastrea 102

debile, Flagellum Milne Edwsrds \& Haime 1848

= Truncatoflabellum spheniscus [23] 129

debilis, Cladocara 102

decactis, Madracis 62

decadia, Cyphastrea 103

decamera, Stenocyathus Ralph \& Squires 1962 = S. vermiformis [107] 130

decipiens, Achatinella 21

decipiens, Acropara Brook = A. rabusta [65] 67

decipiens, Lepidopara 56

decipiens, Paraerrina 56

decora. Achatinella 21

decussata, Pavona 81

decussata, Psammocara 77

deformis, Acropora (Dana 1846) = A. danai 66

defamis, Favia 106

deformis, Goniastrea 107

deformis, Parites 74

delicata, Epiablasma sulcata $=E$. sulcata perabliqua 18

delicata, Javania (Yabe \& Eguchi 1942) $=J$. cailleti (23] 128

delicalula, Acropora $($ Brook 1893) = A. selaga [65] 67

delicatula, Antipathes 36

delicarum, Desmaphyllum Yabe \& Eguchi 1941

= Javania cailleti [23] 128

Deltocyathoides Yabe \& Eguchi 1932 = Delsocyathus 117

Deitocyathus 117

deludens, Flabellum 127

demani, Acropora 66

dendritica, Stylophara Nemenzo $=$ S. pistillata [79] 64

Dendracara 103

Dendrogyra 112

Dendrophyllia 132

dendrostylus, Lepidopora 56

dendrum, Acropara 66

dendyi, Errina 54

dennanti, Fungiacyathus 83

dens, Flabellum 127

densa, Antipathes 36

densa, Poriles 74

densicaulis, Stylaster 58

dentata, Anthemiphyllia 113

dentata, Astrangia 89

dentata, Balanophyllia 131

dentata, Fungia Dana $1846=F$. fungiles [44] 85

dentata, Sandalolitha 88

dentatus, Stylaster 58

denifarmis, Placotrochides Alcock 1902

= Premacyathus dentiformis [107] 124

deniformis, Premocyathus 124

dentigera, Fungia Leuckan $1841=F$. scutaria $[44,65] 86$

derasa, Tridacna 16

desbonni, Anripathes $=$ Stichopathes desbonni 45

desbonni, Cirrhipathes Duchassaing \& Michelotti 1864 = Stichopathes desbanni 45

desbonni, Stichopathes 45

deshayesiana, Acanthophyllia (Michelin 1850)

= C. lacryalis [2] 95

deshayesiana, Acanthophyllia 95 
deshaysiana, Caryophyllia Michelin 1850

= Cynarina lacrymlis [2] 96

desmophylloides, Balanophyllio 131

Desmophyllum 118

diadema, Suephanocyathus 125

diaphana, Dendrophyllia Dana 1846

$=$ ?Tubastrea coccinea [50] 135

diophana, Tubastroea 135

Diaseris 84

Diaseris Milne Edwards \& Haime $1849=$ ?Fungia 85

Dichocoenia 113

dichotoma, Antipathes 36

dichotoma, Millepora 51

dichotoma, Sporadopora 57

Diechorea Tenison Woods $1879=$ Alveopora 72

difluens, Pavona 81

diffusa, Acropora 66

diffuso, Balanophyllia 131

diffusa, Lepidopara 56

diffusa, Oculina 92

digitata, Leptoseris Vaughan $1907=$ L. papyracea $[35,65] 80$

digilato, Montipora 70

digitata, Psammocora 77

digitata, Stylophora (Pallas 1766) $=S$. pistillata 64

digitifera, Acropora (Dana 1846) = ?A. humilis [64] 66

digitifere, Acropora 66

dilatato, Balanophyllia 131

dilatata, Pavona Nemenzo $=P$. danai $[79] 81$

dilitota, Montipora 70

diminuta, Lobophyllia 97

diminuta, Pavona 81

dineta, Balanophyllia 131

diomedeae, Acropora 66

diomedeae, Acroporo Vaughan $1906=$ ?A. nasuta 67

diomedeae, Balanophyltia 131

diomedeae, Caryophyllia 115

diomedeae, Crispatotrochus (Vaughan 1907)

= Cyathoceras rubescens 117

diomedeae, Pocilloporo 63

Diploastrea 104

Diploria 104

Dipsastrea Blainville 1830 = Favia 105

discus, Leptopenus 82

dispar, Acropora Nemenzo $=$ A. grandis [79] 66

dispar, Distichopora 54

dispar, Podabacia Verrill 1901

= Lihophyllon undulatum [44] 87

disparata, Turbinaria Nemenzo $=$ T. reniformis [79] 136

dispersa, Phyllangia 90

dissecta, Antipathes Duchassaing \& Michelotti 1864

$=$ A. pennacea 38

dissimilis, Stichopathes Roule $1902=$ S. filiformis 45

disticha, Acropora Brook = A. nasuta [65] 67

Distichocyauhus Brook 1893 = Acropora 65

Distichopora 54

distinctum, Flabellum Milne Edwards \& Haime 1848

$$
=F \text {. pavoninum [23] } 127
$$

distona, Caulastrea Dana $1846=$ C. furcata [97] 102

distona, Cycloseris (Michelin 1843)

= Diaseris distorta [79] 85

distorza, Diaseris 85

distorta, Fungia Michelin 1843 = Diaseris distorta 85

divaricata, Acropara 66

divaricala, Montipora Brüggemann $=M$. digitata $[65] 70$

divaricala, Pavona 81

divaricala, Poriles 74

divaricata, Psammocora Gardiner = P. contigua $[65] 77$

divergens, Stylaster 58

diversa, Acropora (Brook 1893) = A. secale [65] 67 diversa, Cirthipathes Brook $1889=$ C. spiralis 43

diversa, Pectinia Nemenzo \& Ferraris $=P$. teres [79] 94

diversa, Stichopathes $($ Brook 1889) $=$ Cirmhipathes spiralis 43

diversidens, Fungia Milme Edwards \& Haime 1851

= Heliofungia actinifomis [44] 86

divisa, Euphyllio 119

djiboutiensis, Goniopora 73

doderleini, Fungia Marenzeller 1907

$$
\text { = Cycloseris doderleini } 84
$$

Doderleinia Gardiner 1909 = Sandalolitha [44] 88

doederteini, Cantharellus (Marenzeller 1907)

$=$ Cycloseris doederleini 84

doederteini, Cycloseris 84

doherryi, Troides 12

doherryi, Troides rhadamantus $=T$. dohentyi 12

dolabelloides, Lexingtonia 19

dolabelloides, Quadrula = Lexingtania dolabelloides 19

Domaseris Quelch $1886=$ Leptoseris 80

donei, Acropora 66

donnani, Coscinoraea Gardiser $=C$. monile [65] 76

doreyensis, Favia Milne Edwards \& Haime $=F$. pallida $[65] 106$

dromas, Conchodromus = Dromus dromas 18

dromas, Dromus 18

Dromus 18

dubia, Antipathes 36

dubia, Tylopathes Brook 1889 = Antipathes dubia 36

duchassaingi, Stylaster 58

duerdeni, Pavono 81

duerdeni, Porites 74

dumosa, Acropora 66

dumosa, Goniocorella 119

duncani, Antillia Yabe \& Sugiyama 1931

= Trachyphyllio geofforyi [2] 112

Duncania Pourtalès $1874=$ Gardineric [23] 128

Duncanopsammia 132

Dunacyathus 118

duofociata, Goniopora Thiel $=$ G. planulata [65] 73

dura, Conopora 53

durotrix, Hoplangia 120

durus, Fungiacyathus 83

durus, Fungiacyathus Keller $1976=$ ?F. marenzelleri [?23; 83

Dysnomia 18

ebumea, Galarea Pourtalès $1871=$ Javania cailleti $[107] 128$

ebumea, Javania (Moseley 1881) =J. cailleti [23] 128

ebumeum, Desmophyllum (Pouralès 1871)

= Javania coilleti $[23,107] 128$

eccentricus, Deloocyathus 118

Echinastrea Blainville $1830=$ Echinopora 104

echinata, Acanthastrea 95

echinata, Acropora 66

echinata, Cladopsammia 132

echinata, Clenactis 83

echinata, Fungia (Pallas 1766) = Clenactis echinata [44] 83

echinata, Madrepora Pallas 1766 = Ctenactis echinata [44] 83

echinava, Stellapora 57

echinata, Stenahelia 57

echinatus, Pliobothrus 57

echinatus, Stylaster Broch $1936=$ S. filogranus [18] 58

echinatus, Zoopilus 88

Echinaphyllia 93

Echinopore 104

echinoporoides, Echinophyllia 93

echinulata, Caulastrea 102

echinulata, Porites 74

echinulata, Stichopathes 45

eclipsensis, Goniopara 73

edgariana, Fusconaia 19 
edwandsi, Goniastrea 107

edwandsi, Leptoseris 80

edwandsi, Lithophyllon (Rousseau 1854)

= ?Leptopelis edwandsi [44] 87

edwandsi, Lithophyllon 87

edwandsi, Montipora 70

Edwardsotrochus 118

efflorescens, Acropara (Dana 1846) = A. cytherea [65] 66

eflorescens, Montipora 70

effuens, Acropora 66

effusa, Acropora (Dans) = A. diginfera [65] 66

effusa, Montipora 70

eguchii, Balanophyllia 131

eguchii, Stylaster 58

ehrenhergana, Leptastrea Milne Edwards \& Haime =L. purpurea [65] 108

ehrenbergi, Favia (Klunzinger) = F. favus [65] 106

ehrenbergi, Montipora 70

ehrenbergii, Herpetolithus Leuckan 1841

= Crenoctis echinato [44] 83

eibli, Acropora Pillai \& Scheer = A. granulosa $[65] 66$

elassotomus, Stylaster 58

elegans, Acharinella 22

elegans, Acropora 66

elegans, Antipathes 36

elegans, Balanophyllia 131

elegans, Cycloseris (Verrill 1870) $=$ C. curvala 144] 84

elegans, Flabellum Milne Edwards \& Haime 1848

= Truncatoflabellum candeanum [23] 129

elegans, Fungia Verrill $1870=$ Cycloseris curvata [44] 84

elegans, Pocillopora Dana $1846=$ P. vernucosa ]65] 63

elegans, Siephanophyllia 82

elegans, Stylaster Duchasssaing \& Michelotti 1864

= S. duchassaingi $[14,18] 58$

elegans, Stylaster Verrill $1864=S$. sanguineus [14] 59

elegans, Turbinaria 136

elegans, Tylopathes Brook 1889 = Antipathes elegans 36

elegantula, Acropora (Ortmann) = A. aculeus [65] 65

elephantous, Mycedium 93

elliprica, Acropora 66

elongala, Favia 106

elongata, Pectinia 94

elongata, Thecopsammia 134

elongatum, Flabellum 127

elschneri, Montipora 70

elseyi, Acropora 66

elraninae, Caryophyllia 115

eminens, Turbinario Nemenzo $=T$. irregularis [79] 136

Enallopsammia 133

Endocyathopora 118

Endopachys 133

Endopsammia 133

ephyala, Caryophyllia 115

Epioblasma 18

epiphanes, Omithoptera victoriae 11

epithecala, Caryophyllia 115

equatorialis, Astrangia 89

equiserialis, Coenopsammia 132

ericoides, Antipathes 37

ericoides, Arachnopalhes (Pallas 1766)

= Antipathes ericoides 37

eridani, Favia Umbgrove $1940=F$. valenciennesi [97] 106

eridani, Porites 74

erinaceus, Antipathes 37

erinaceus, Aphanipathes Roule 1905

= Antipahes erinaceus 37

erasa, Cyclaseris (Döderlein 1901) ?= C. tenuis [44] 84

erosa. Cyclaseris 84

erosa, Fungia (Dōderlein 1901) = Cycloseris erosa 84 erosa, Porites 74

erotema, Bathypathes 41

Errina 54

Errinapora 55

Eminapsis 55

erubescens. Stylaster 58

erythraea, Acanthastrea (Klunzinger 1879)

= Symphyllia erychraea 99

erythraea, Acropara (Klunzinger) = A. humilis [65] 66

erythraea, Montipora Marenzeller 1906

$=M$. ehrenbergi $[64 \mid,=M$. aequituberculata $[65] 70$

erythraea, Symphyllia 99

Enythrastrea 105

Esturgeon Noir 1

etheridgei, Notophyllia 134

ewantha, Bathypathes 41

eueides, Crypthelia 53

Eumadrepora Brook 1893 = Acropora 65

euphorion, Omithoptera priamus 10

Euphyllia 118

Eupsammia 133

eupsammides, Heterocyathus 119

eupsammides, Heteropsammia 133

eupteridea, Antipaches 37

eupteridea, Aphanipathes (Lamouroux, Bory de Saint-Vincent

\& Deslongchamps 1824) = Antipathes eupteridea 37

europaea, Balanophyllio 131

eurysepta, Plerogyra 123

eurystoma, Acropora 66

Eusmilia 119

Eusthenotrochus Wells $1945=$ Sphenotrochus 124

evermanni, Porites 74

excesa, Millepora 51

excavala, Porites Verrill $=P$. lobota 75

excavalus, Sphenotrochus 124

excelsa, Alveopora 72

exerta, Physogyra 123

exesa, Coscinaraea 76

exesa, Hydnophora 100

exesa, Psammocora Dana = Coscinaraea exesa 76

exiguc, Acropora (Dana 1846) $=$ ?A. formoso [65] 66

exigua, Lophelia Pourtalès 1871

= Madrepora exigua [107] 92

exigua, Madrepora 92

exigua, Plarygyra Nemenzo 1959

= Goniastrea favulus [97] 107

exilis, Acropora (Brook) = A. elseyi [65] 66

exilis, Porites 74

eximia, Goniastrea (Dana 1846) $=$ G. retiformis 107

eximius, Stylaster 58

eximius, Sylaster Kent $1871=S$. duchassaingi $\mid 14,18] 58$

expanda, Stylophora Nemenzo = S. pistillota 179] 64

exponsa, Astreopora 69

expansa, Leiopathes 43

expansa, Montipora (Dana 1846) $=$ M. hispida 71

Explanaria Lamarck 1816 = Turbinaria 135

explanaia, Alveopora 72

explanata, Astreopora 69

explanata, Leptoseris 80

explanata, Leploseris Yabe \& Sugiyama 1941

$$
?=\text { L. scabra [35] } 80
$$

explanata, Montipora 70

explanatus, Stylaster (Kent 1871) $=S$. nobilis [14] 59

explanulata, Montipora 70

explanulasa, Pavana 82

explanulata, Psammocora 77

exquisita, Acropora 66

exseria, Montipora 70

exseria, Porites 74 
exusta, Cladangia 89

eydouxi, Pocillopora 63

facetus, Labyrinthocyathus 120

fallosocialis, Bothypsammia 131

fasciatus, Trochocyathus 126

fascicularis, Galaxea 91

fascicularis, Lepidotheca 56

fasciculata, Millepora 51

fastigiata, Eusmilia 119

faulkneri, Tubastraea 135

faustinoi, Poriles (Hoffmeister 1925) = ?P. nus [79] 75

faustinoi, Poriles 74

Fovio 105

favistella, Goniastrea (Dana 1846) = G. pectinata 107

Favites 106

favosa, Pocillopora Ehrenberg = P. damicomis [65] 63

favosa, Porites 74

Favositopora Saville Kent $1870=$ Alveopora 72

favulus, Goniastrea (Dana 1846) = ?G. pectinato 107

favulus, Goniastrea 107

favus, Favia 106

favus, Madrepora Forskál 1775 = Favia fovus [97] 106

fecunda, Anomocora 114

fecunda, Coenosmilia (Pourtalès 1871)

= Anomocora fecunda 114

fecunta, Parasmilia (Pourtalès 1871)

= Anomocora fecunta [107] 114

fenestrata, Alveopora 72

fenestrata, Errinopsis 55

femandezi, Antipathes 37

femandezi, Parantipathes (Pourtalès 1874)

= Antipathes femandezi 37

ferox, Mycetophyllia 98

ferrari, Troides helena 13

fieldi, Fungia Gardiner $1909=F$. scruposa [44] 86

fijiensis, Alveopora Hoffmeister $1932=$ A. spongiosa 67

filicasa, Favites (Dana 1846) $=$ F. abdita 106

filiformis, Cirrhipathes Gray $1868=$ Stichopathes filiformis 45

filiformis, Srichopathes 45

filix, Anripathes Pourtalès $1867=$ Aphanipathes filix 41

filix, Aphaniparhes 41

filix, Parantipathes (Pourtalès 1868) = Aphonipothes filix 41

filogranus, Stylaster 58

fimbriata, Euphyllia 119

fiordensis, Antipathes 37

fiordlandica, Paryphania 23

fiordlandica, Powelliphanta = Paryphanta fiordlandica 23

fima, Anacropora Nemenzo \& Ferraris = A. forbesi [79] 68

fisheri, Distichopora Broch $1942=$ D. vialacea [14] 54

Fissicella Dana 1848 = Favia 105

fissilis, Fungiacyathus 83

fissilis, Schizocyathus 130

fissipara, Dendrocora 103

fissispina, Schizoculina 92

fissurata, Errina 55

fistula, Dendrophyllia 132

fistulasa, Lobophyllia (Milne Edwards \& Haime)

$$
=L \text { corymbosa [65] } 97
$$

Rabellata, Erythrastrea 105

flabellata, Montipora 70

flabellata, Stylophora Quelch = S. pistillato [79] 64

fabelliformis, Acropora 66

Rabelliformis, Rhizotrochus 129

flabelliformis, Stylaster 58

fabelliforms, Antillia Yabe \& Sugiyama

= Trachyphyllia geafforyi [2] 112

flabellum, Antipathes 37

fabellum, Tylopathes (Pallas 1766) = Antipothes flobellum 37
Flabellum 126

Alagellum, Cirthipathes Brook 1889

= Stichopathes flagellum 45

Ragellum, Stichopathes 45

favescens, Omithoptera paradisea 10

flavicollis, Troides amphrysus 12

flavidior, Omithoptera chimaera 10

flexuosa, Favites 106

flexuosum, Flabellum 127

floreana, Tubasiraea 135

forentina, Dysnomia forentina = Epioblasma florentina 18

florentina, Epioblasma 18

forentina, Epioblasma florentina $=$ E. florentina 18

florentina, Plagiola = Epioblasma florensina 18

florida, Acropora 66

florida, Montipora 70

Aoridana, Balanophyllia 131

foridana, Crypthelia 53

Naridanum, Flagellum 127

flos, Phacelocyothus 122

foweri, Montiporo 70

foeniculacea, Antipathes Pallas $1766=$ A. dichotoma 36

foeniculum, Antipathes Lamarck $1816=$ A. dichotoma 36

foliacea, Distichopara 54

foliala, Coscinaraea Nemenzo = Leplaseris yobei [79] 80

foliasa, Haliglossa Ehrenberg 1834

= Herpolitha limax [44] 87

foliosa, Leptoseris 80

foliosa, Leptoseris Dineson $1980=$ ?L. tenuis [65] 80

foliosa, Montipora 70

foliosa, Pachyseris 81

foliosa, Pavono 82

Falioseris Rehberg 1892 = Leptoseris 80

folium, Psammocora 78

folliculus, Peponocyathus 122

folliculus, Stephanophyllia Pourtales 1868 = Peponocyathus folliculus [23] 122

forbesi, Anacropora 68

foresti, Caryophyllia 115

formosa, Acropora 66

formosa, Callogyra 112

formosa, Crypthelia 53

formosa, Leptopsommia 134

formosa, Madracis 62

formosa, Pavone 82

formosa, Podabacia Yabe and Sugiyama 1932 $=$ Lithophyllon undulatum [44] 87

formosissima, Letepsammia 82

formosissima, Stephanophyllia Moseley 1876 = Letepsammia formosissima [23] 82

formosum, Truncalofiabellum 129

fomosus, Scleropoges 2

forskaliana, Echinopora 105

forskalii, Acropora 66

forsteri, Neoceratodus 1

fossala, Coscinaraea (Dana 1846) = C. columna 76

fossulus, Trochocyathus 126

foveolato, Montipora 70

foxi, Crispatotrochus (Dutham \& Bamard 1952) = Cyathoceras foxi 117

foxi, Cyathoceras 117

fragile, Flabellum 127

fragile, Flabellum Cairns $1977=$ ?F. floridanum 127

fragilis, Adelopora 52

fragilis, Agaricia 79

fragilis, Antipathes 37

frogilis, Antipathes Gravier $1918=$ A. lento 38

fragilis, Crypthelia 53

frogilis, Distichopora Quelch $1885=$ D. gracilis [14] 54 
fragilis, Favia (Dana 1846) $=F$. speciosa 106

fragilis, Fungia (Alcock 1893) = Diaseris fragilis 85

fragilis, Fungiacyathus 83

fragilis, Leptoseris 80

fragilis, Pohymyces 129

fragilis, Pteropathes Brook 1889 = Antipathes fragilis 37

Fragilocyathus Yabe \& Eguchi 1932 = Aubocyathus 114

fragosa, Poriles Dana $1846=P$. australiensis [65] 122

fragum, Favia 106

fralinae, Fungia 85

freycineti, Diaseris Milne Edwards \& Haime 185

$$
=\text { Cyclaseris sinensis [44] } 84
$$

friabilis, Montipora 70

frondens, Turbinaria 136

frondifera, Pavona 82

frondosa, Pocillopora 63

frandosa, Psammocora Verrill $1872=P$. contigua 77

frustra, Placotrochides 128

fruticosa, Antipathes 37

fruticosa, Aphanipathes (Gray 185T) = Antipathes fruticosa 37

fruticosa, Goniopora 73

fruticasa, Montipora 70

fulgens, Acharinello 22

fulvacea, Distichopora Michelin $1862=$ ?D. violacea [14] 54

fungia, Polyphyllia Dana $1846=P$. talpina $[44] 88$

Fungia 85

Fungiacyathus 83

fungiformis, Montiporo Bernard $=M$. monasteriata [65] 71

fungites, Fungia 85

fungites, Madrepora Linnaeus 1758 = Fungia fungites [44] 85

fungulus, Stephanophyllia 82

funicolumna, Conotrochus 116

furanaensis, Polycyathus 124

furcata, Antipathes 37

furcata, Caulastrea 102

furcala, Porites 74

fuscobasis, Achatinella 22

fuscomarginata, Phyllangia Kunzinger 1879

= Polycyathus fuscomarginalus [107] 123

fuscomarginatus, Polycyathus 123

Fusconaia 19

fuscus, Placotrochus 128

gaditana, Dendrophyllia 132

gaimardi, Montipora 70

gaimardi, Porites 75

galapagense, Desmophyllum Vaughan 1906

$$
\text { = Javania cailleti [25] } 128
$$

galopagensis, Antipathes 37

galapagensis, Balanophyllia 131

galapagensis, Crispatotrochus 117

galopagensis, Javania (Vaughan 1906) $=J$. cailleti [25] 128

galapagensis, Madrepora 92

galapagensis, Pavona 82

galapagensis, Stylaster 58

galapagoensis, Amphelia (Vaughan 1906) = A. oculata 67

galatheae, Bathypathes 42

Galaxea 91

galeata, Porites 75

galeriformis, Palyphyllia Dana 1846

$$
=P \text {. novachibemiae }[44] 88
$$

gallensis, Antipathes 37

gandineri, Cimhipathes Forster Cooper $1903=$ C. anguina 42

gandineri, Cyphastrea 103

gandineri, Flabellum 127

gandineri, Leptoseris 80

gandineri, Pavona (Horst) = Leptoseris gardineri [65] 80

gandineri, Sphenotrochus 125

gardineri, Trochocyathus 126
Gandineria 128

Gandineroseris 79

gardnerensis, Astrangia Durham \& Barnard 1952

$$
\text { = A. equatorialis } 89
$$

gasti, Thalamophyllia 125

gebeensis, Omithoptera priamus 10

geertsi, Caecobarbus 2

gemmacea, Echinopona 105

gemmae, Pachyseris 81

gemmascens, Stylaster 58

gemmifera, Acropora (Brook 1892) = ?A. humilis [64] 66 gemmifera, Acropora 66

gemmifera, Balanophyllia 131

gemminata, Heteropsammia Verrill 1870

$=H$. cochlea [65] 133

Gemmipora Blainville 1830 = Turbinoria 135

Gemmulatrochus Duncan 1878 = Hoplangia 120

geoffroyi, Trachyphyllia 112

gephura, Sibopathes 44

gendae, Rhizasmilia 124

gibsonhilli, Porites Wells $1950=P$. cylindrica 74

gigantea, Conopora 53

gigantea, Crypthelia 53

gigantea, Fungia Dana 1846 = Clenactis echinata [44] 83

gigantea, Pavona 82

gigas, Alveopora 72

gigas, Arapaima 2

gigas, Pangasianodon 2

gigas, Strombus 24

gigas, Tridacna 16

gilchristi, Sphenatrochus 125

gilliesi, Paryphania 23

gilliesi, Powelliphanta $=$ Paryphanta gilliesi 23

gippslandicus, Truncaloflabellum 129

glaberrima, Antipathes Esper 1794

$$
\text { = Leiopathes glaberrima } 43
$$

glaberrima, Leiopathes 43

glabra, Errina Pourtalès 1867 = Lepidopora glabra [18] 56

glabra, Lepidopora 56

glabra, Leptoseris 80

glabra, Oxypora 94

glabrescens, Euphyllia 119

glans, Carunculina 20

glans, Fungia Dana $1846=$ Cycloseris cyclolites [44] 84

glauca, Acrapara 66

glaucopsis, Diploastrea (Dana 1846) $=$ D. heliapora 104

glebulenta, Crypthelia 53

globiceps, Acropora (Dana 1846) = A. humilis 66

globularis, Turbinaria Bernard = T. stellulata [65] 136

glochiclados, Acropora 66

glassopoma, Crypthelia 53

glutinata. Antipathes 37

goliath, Omithopiera 10

goliath, Omithoptera goliath 10

goliath, Schoenbergia = Omithoptera goliath 10

gombergi, Thalamophyllia 125

Gombessa 1

gomezae, Zoopilus Nemenzo $1980=Z$. echinalus $[44,79] 88$

gonagra, Acropora 66

gonagra, Psammocora Klunzinger $=P$. contigua $[65] 77$

Goniastrea 107

Goniacorella 119

Goniocyathus Yabe \& Eguchi 1932 = Caryophyllia 114

Goniopora 72

goodei, Stephanocoenia (Verrill 1900) $=S$. intersepta 61

Goreaugyra Wells 1973 = Meandrina 113

gracile, Flabellum 127

gracilis, Acropora (Dana 1846) = ?A. formosa 66

gracilis, Acropora 66 
gracilis, Anacropora 68

gracilis, Antipathella (Gray 1860) = Stichopathes gracilis 45 gracilis, Anripathes G. von Koch $1889=$ ?A. lenta 38

gracilis, Antipathes Gray $1860=$ ?A. atlantica 35

gracilis, Antipathes Gray $1857=$ Stichopathes gracilis 45

gracilis, Astreopora 69

gracilis, Dendrophyllia 132

gracilis, Distichopora 54

gracilis, Emina 55

gracilis, Goniopora 73

gracilis, Leptoria (Dana) = L. phrygia 108

gracilis, Montipora 70

gracilis, Pliobothrus 57

gracilis, Stichopathes 45

gracilis, Stylaster 58

grahamae, Agaricia 79

grandiflora, Antillia Gerth 1921 = Cynarina lacrymlis |2| 96

grandiflora, Antipathes 37

grandifolia, Montipora 70

grandis, Acropora 66

grandis, Antipathes 37

grandis, Balanophyllia 131

grandis, Caryophyllia 115

grandis, Hydnophora (Gardiner 1904)

$=? H$. exesa $[65,97] 100$

grandis, Hydnophora 100

grandis, Pocillapora Dana $1846=$ P. eydouxi [65] 63

granifera, Montipara 70

granulata, Montipora Bemard = M. informis [65] 71

granulata. Phyllangia 90

granulosa, Acropara 66

granulosa, Distichopara Quelch $1885=$ D. gracilis [14] 54

granulosa, Fungia 85

granulosa, Lepidopora 56

granulosa, Montipora 71

granulosus, Fungiacyathus 83

granulosus, Stylaster 58

gravida, Acropara (Dana) = A. florida [65] 66

gravida, Favia 106

gravieri, Leptoseris van der Horst 1922

$$
\text { = L. hawaiiensis [35] } 80
$$

gravieri, Physogyra 123

gravieri, Plesiastrea (Vaughan 1918) = P. versipora 111

gravis, Fungia 85

gravis, Fungia Nemenzo $1955=? F$. paumatensis $179 \mid 85$

grayi, Acanthocyathus = Caryaphyllia grayi 115

grayi, Antipathes 37

grayi, Caryophyllia 115

grayi, Endopachys 133

grayi, Paratylopathes (Roule 1902) = Antipathes grayi 37

grayi, Tylopathes Roule 1902 = Antipathes grayi 37

grimaldii, Leiopathes 44

grisea, Montipora 71

gubernaculum, Dysnomia torulasa

= Epioblasma torulosa gubemaculum 18

gubemaculum, Epioblasma torulosa 18

gubemaculum, Plogiola torulosa

= Epioblusma torulosa gubemaculum 18

gubemaculum, Truncilla torulosa

= Epioblasma torulosa gubernaculum 18

guentheri, Stylocaeniella 61

guppyi, Montipora Bernard = M. spumosa [65] 71

Guynia 130

Gyropora 55

Gyrosmilia 119

hachijoensis, Lepidotheca 56

haddoni, Porites Vaughan $1918=P$. lutea 75

hadros, Balanophyllia 131 hahajimaensis, Coscinaraea 76

haimeana, Psammocara 78

haimei, Acropora (Milne Edwanda \& Haime 1860)

$$
\text { = A. yongei }[65,85] 68
$$

haimei, Fungia Verrill $1864=F$. fungites [44,65] 85

halicora, Favia 106

halicora, Favites 106

Haliglossa Ehrenberg $1834=$ Herpalitha [44] 86

haliphron, Troides 12

haliphron, Troides haliphran 12

Halomitra 86

Haloseris Milne Edwarda \& Haime $1849=$ Leptoseris 80

hancocki, Aphanipathes 41

hancocki, Sphenotrochus 125

hannibali, Dendrophyllia 132

hanno, Troides oblongomaculatus 13

hanzawai, Stylocoeniella (Yabe \& Sugiyama 1933)

$=S$. armasa 61

Haplaphyllia Pourtalès 1868 = Gardineria [23] 128

hameri, Flabellum Gardiner 1929

= Monomyces rubrum [23] 128

hartii, Mussismilia 98

hassi, Symphyllia Pillai \& Scheer $=S$. valenciennesii [65] 99

hastatus, Platytrochus 123

hataii, Lobophyllia 97

hattorii, Stylaster 58

haugumei, Trogonoptera braakiana 12

hawailensis, Balanophyllia Vaughan $1907=$ B. comu 131

hawaiiensis, Caryophyllia 115

hawaiiensis, Fungiacyathus (Vaughan 1907)

$=F$. fragilis [23] 83

hawaiiensis, Gardineria 128

hawaitensis, Leptastrea (Vaughan 1907) = L. batrae 108

hawaiiensis, Leploseris 80

hawaiiensis, Porites 75

hawaiiensis, Porites Vaughan $1907=$ ?P. rus 75

hayamaensis, Phyllangia 90

hebes, Acropora (Dana 1846) = A. aspera 65

hecuba, Omithoptera priamus 10

hedleyi, Tremaloirochus 125

helena, Traides holena 13

helianthoides, Favia 106

hetianthoides, Plesiastrea Wells 1954 = Favia laxa [97] 106

Heliastrea Milne Edwards \& Haime 1848 = Montastrea 109

helicanoides, Troides helena 13

Heliofungia 86

heliopora, Diploastrea 104

Heliopora 60

Hetioseris Milne Edwards \& Haime $1849=$ Leptoseris $\mathbf{8 0}$

hemisph(a)erica, Psammoseris 134

hemprichi, Favites (Ehrenberg) $=F$. complanata [65] 106

hemprichi, Pocillopora Ehrenberg $=P$. vemucosa [65] 63

hemprichii, Acanthastrea 95

hemprichii, Acropora 66

hemprichii, Lobophyllia 97

hephaesus, Troides helena 13

herdmani, Cyachotrachus Boume 1905 $=2$ Tropidacyathus pileus 126

heronensis, Porites 75

heronensis, Turbinario 136

Herpetoglossa Wells $1966=$ Ctenactis [44] 83

Herpetolitha Milne Edwards \& Haime 1851 = Herpolitha [44]86

Herpolitha 86

hesiodus, Troides $=T$. thadamanius 13

heteraclados, Acropora 66

Heterocjathus 119

Heteropora Ehrenberg 1834 = Acropora 65

Heteropsammia 133 
helerorhodzas, Antipathes Forster Cooper 1909

= Bachypathes heterorhodzos 42

heteromodzas, Bathypathes 42

heterasticha, Hexapathes 43

hexagonalis, Cycloseris 84

hexaganalis, Fungia (Milne Edwards \& Haime 1848)

= Cycloseris hexaganalis 84

hexagonalis, Galaxea $=G$. fascicularis $[59,65] 91$

hexagonus, Bathytrachus Gravier 1915

= Fungiacyathus marenzelleri [107] 83

Hexapathes 43

hicksani, Errina 55

hicksoni, Lepidopora 56

higginsii, Lampsilis 19

hillae, Acanthastrea 95

Hillopathes 43

hippopus, Hippopus 16

Hippopus 16

hirsuta, Echinophyllia 93

hirsula, Mantipara 71

hirsutissima, Echinopora 105

hina, Antipathes 37

hina, Parantipathes (Gray 1857) = Antipathes hirta 37

hirtella, Sclerhelia 92

Hinuda 15

hispida, Acropora (Brook) = A. cuneata [79] 66

hispida, Mantipora 71

hispida, Mussismilia 98

hispidula. Mantastrea (Verrill 1901) $=$ M. annularis 109 hispidus, Paurialocyathus 130

hochstetteri, Paryphanta 23

hachstetteri, Powelliphanta = Paryphanta hochsteueri 23

hodgsoni, Polycyathus 124

hoffmeisteri, Culicia 89

hoffmeisteri, Flagellum 127

haffmeisteri, Mantipora 71

Halcotrochus 120

hombroni, Favia (Rousseau 1854) = F. sielligera [65] 106

hombroni, Goniastrea Rousseau

$$
\text { = Favia sielligera }[59,65] 106
$$

homeris, Papilia 11

Hamophyllia Brüggemann $1877=$ Scolymia 98

hondaensis, Asirangia Durham \& Barnard 1952

$$
\text { = Polycyathus hondaensis [25] } 124
$$

hondaensis, Palycyathus 124

hanrathiana, Traides vandepolli 13

Hoplangia 120

Horastrea 77

harizantalata, Poriles 75

harizantalis, Astreopora Bemard = A. listeri [65] 69

harolgium, Stylaster 58

harologium, Canyaphyllia 115

horrescens, Acrhelia 90

horrida, Acrapara 66

horrida, Echinopara 105

horrida, Fungia 85

horrida, Lepidotheca 56

horsti, Dendrophyllia 132

hospilon, Papilio II

huluensis, Favia Gardiner 1904

$$
=F \text {. pallida }[65] / F \text {. roulosa [98] } 106
$$

humilis, Acrapora 66

humilis, Agaricia 79

humilis, Antipathes Pourtalès $1867=$ Aphanipathes humilis 41

humilis, Aphanipathes 41

humilis, Paracyathus 122

hyacinihus, Acropora 66

hyades, Solenastrea 111

Hydnophora 99
Hydnophorella Delage \& Hérouard 1899 = Hydnophora 99 hydra, Fungiacyathus 83

hypnoides, Antipathes 37

hypnoides, Tylopathes Brook 1889 = Antipathes hypnoides 37

hypocoelus, Leptopenus 82

hypolitus, Ripponia $=$ Troides hypalitus 13

hypolitus, Troides 13

hypolitus, Troides hypolitus 13

hystrix, Acropara (Dana 1846) = A. cerealis 66

hystrix, Galaxea (Dans 1846) = G. fascicularis 91

hystrix, Seriatopara 64

ibericus, Stylaster 58

Idiotrochus 120

Ikan Temoleh 2

ikarus, Troides haliphron 12

imbricalus, Stylaster 58

immersa, Colangia 89

immersa, Leptastrea Klunzinger $1879=$ L. bottae [65] 108 impensum, Flabellum 127

imperfecta, Acrapara Nemenzo $=$ A. latistella [79] 66

imperialis, Balanaphyllia 131

imperialis, Teinopalpus 12

implicata, Acropora 66

inaequalis, Leptastrea 108

inaequalis, Leptastrea Klunzinger $1879=$ ? L. bottae [65] 108 incisa, Balanophyllia 131

incagnita. Montipora 71

incompletus, Stylaster 59

incanstans, Montipora Nemenzo $=M$. altasepta [79] 70

inconstans, Truncatoflabellum 129

inconsua, Lepidotheca 56

incrassala, Montipora 71

incrassatus, Stylaster (Eguchi 1942)

$=S$. bareopacificus [14] 58

incrustans, Astreopora 69

incrustans, Leptaseris 80

incrustans, Montipara Brüggemann

$=$ M. manasteriala [65] 71

incrustatum, Truncataflabellum 129

indica, Acropora 66

indica, Dendrophyllia 132

indica, Horastrea 77

indica, Stichopathes Schultze $1903=$ S. gracilis 45

indicus, Paracyathus 122

indistincta, Antipathes 37

indistincta, Aphanipathes (van Pesch 1914)

$=$ Antipathes indistincta 37

Indophyllia 96

Inferialabiata 55

inflata, Pyrophyllia Hickson 1910

= Grynia annulata [23] 130

informis, Mantipora 71

infarmis, Pocillopara 63

infundibuliformes, Antillia Gerth 1921

= Trachyphyllia geafforyi [2] 112

infundibulum, Trochopsammia 135

inardinata, Coenasmilia 116

inamala, Caryophyllia 115

inornatus, Crispatotrochus Tenison-Woods 1878

= Cyathoceras inomarus 117

inomatus, Cyathaceras 117

inomatus, Stylaster 59

insignis, Acropora 66

insignis, Acropora Nemenzo $1967=$ ?A. selaga 67

insignis, Javania 128

inskipi, Caryaphyllia Duncan $1873=$ C. abyssarum [107] 114

insalita, Crypthelia 53

integra, Fungia Dana $1846=F$. repanda [44] 85 
interjecta, Madracis 62

intermedia, Acropora (Brook 1891) = A. nobilis [65,85] 67

intermedia, Antipathella Brook 1889

= Anripathes intermedia 38

intermedia, Antipathes 38

intermedia, Pavona 82

intermedia, Quadrula 20

intermedius, Orthonymus = Quadrula intermedia 20

intermedius, Trochocyathus Yabe \& Eguchi 1932

= Tropidocyathus pileus [23] 126

intermupta, Gyrosmilia 119

intermupta, Haliglossa Ehrenberg 1834

= Herpolitha limax [44] 87

intervacans, Emina Naumov 1960

= Errinopora stylifera [14] 55

intricata, Millepara 51

investigataris, Lophelia Aicock 1898

= Madrepora investigatoris [107] 92

investigataris, Madrepara 92

involuta, Padabacia van der Horst 1921

$=P$. cruslacea $[44] 87$

irinae, Leptopenus 82

iris, Troides haliphron 12

irregulare, Flabellum Semper 1872

= Truncaloflabellum irregulare [23] 129

irregulare, Truncatoflabellum 129

irregularis, Acanthastrea Quelch = ?A. echinato [65] 95

irregularis, Acropara (Brook 1892) = A. danai [65] 66

irregularis, Anomastraea 76

irregularis, Crispatatrochus (Caims 1982)

= Cyathoceras irregularis 117

irregularis, Cyathoceras 117

irregularis, Distichopora 54

irregularis, Halamitra Gardiner 1898

= Sandalolitha robusta [44] 88

irregularis, Leptoria 108

irregularis, Pariles 75

irregularis, Truncaloguynia 130

irregularis, Turbinaria 136

isabela, Polycyathus 124

isabellae, Omichoptera victoriae 11

isara, Troides helena 13

ishigakiensis, Acanthastrea 95

Isophyllastrea 96

Isophyllia 97

Isopora Studer 1878 = Acropora 65

italica, Balanophyllia 131

italicus, Deltocyathus 118

iwayamaensis, Porites Eguchi $=$ P. rus [65] 75

japonica, Alveopora 72

japonica, Antilia Yabe \& Sugiyama

= Cynarina lacrymlis [2] 96

japonica, Caryophyllia 115

japonica, Crypthelia 53

japonica, Culicia 89

japonica, Cyphastrea 103

japonica, Lepidatheca 56

japonica, Stephanophyllia Yabe \& Eguchi

= Letepsammia formosissima [23] 82

japonica, Stichopathes Silberfeld 1909

= Antipathes Langibrachiata 38

japonicum, Flabellum 127

japonicus, Deltocyathoides Yabe \& Eguchi 1932

= Peponocyathus australiensis [23] 122

japanicus, Deltocyathus 118

jardinei, Catalaphyllia 116

Javania 128

jefferyi, Solenosmilia Alcock $1898=$ S. variabilis [107] 124 jagashimaensis, Caryophyllia 115

johnsoni, Ceratotrochus Duncan 1882

= Asterosmilia prolifera [107] 1$] 4$

johrsoni, Dendrophyllia 132

jonesi, Montipora 71

juddii, Achatinella 22

jullieni, Probarbus 2

juncea, Achasinella 22

juvenescens, Aulocyathus 114

kabiraensis, Euphyllia 119

kaguya, Troides aeacus 12

Kaiser-l-Hind, Golden 12

Kaiser-J-Hind 12

kawaiensis, Madracis 62

kawaiensis, Madrepora 92

kenti, Acropora (Brook) = A. tenuis [65] 68

kenti, Montigyra 121

kerguelensis, Emina 55

likuti, Idiotrochus 120

kikutii, Placotrochides Yabe \& Eguchi 1941

= Idiotrochus kikurii [23] 120

Kionotrochus 120

kirbyi, Madracis 62

lisstyae/i, Acropora 66

Hunzingeri, Fungia 85

klunzingeri, Fungia Döderlein $1901=$ ?F. horrida [44] 85

klunzingeri, Goniopora 73

knori, Flabellum 127

kondoi, Cyarhoceras Wells 1977

= Labyrinthocyathus kondoi [25] 120

kondoi, Labyrinthocyathus 120

kuehlmanni, Stylophora 64

labiata, Inferiolabiata 55

Laboreli, Dendrophyllia 132

Labrasa, Acropora (Dana 1846) = A. prolifera 67

Labyrinthiformis, Diploria 104

Labyrinthocyathus 120

laccadivica, Favia Gardiner $1904=$ F. pallida [97] 106

lacera, Fungia V:rrill $1866=F$. fungites [44] 85

lacera, Oxypora 94

lacera, Scolymia 99

laciniatum, Flabellum Duncan 1873

$$
=F \text {. macandrewi [107] } 127
$$

Laciniasa, Fungia Boschma 1925 = Diaseris fragilis 85

lacrymalis, Cynarina 96

laciuca, Pectinia 94

lacunosa, Crypthelia 53

laddi, Barabanoia 101

laddi, Favia (Wells 1954) = Barrabattoia laddi 10]

laevifundus, Stephanocyathus 125

Laevigata, Concentrotheca 116

laevigala, Emina 55

laevigatus, Plarytrochus 123

laevigatus, Stylaster 59

laevigatus, Thecocyathus Pourtalès 1871

= Concentrotheca laevigata [107] 116

laevigranulasa, Distichopora 54

laevis, Conopora 53

laevis, Placairochus 128

Lagiola 18

Lajollaensis, Astrangia 89

lamarcki, Agaricia 79

Lamancki, Galarea Milne Edwards \& Haime

$$
\text { = G. asireala [65] } 91
$$

lamarckiana, Mycetophyllia 98

lambersi, Astreopara 69 
lamellata, Cyclohelia 54

lamellifera, Caryophyllia 1 is

lamellina, Plarygyra 111

lamellina, Plarygyra Ehrenberg 1834

$=$ ?P. daedalea $[59,64]$ [11

lamellosa, Echinopora 105

lamellulosum, Flabellum 127

Lamprorichum, Javania 128

Lampsilis 19

langi, Labyrinthocyathus 120

lanuginosa, Montipora Bernard = M. manasieriala [65] 71

laricides, Parantipathes 44

Larix, Antipathes Esper $1794=$ Parantipathes larix 44

lanix, Parantipathes 44

lata, Antipathes 38

lata, Pavona $($ Dana 1846) $=P$. decussata $[65] 81$

Laterorifa, Emina 55

laticastata, Cyphastrea Nemenzo = C. serailio [79] 103

laticostata, Endocyathopora 118

laticostata, Galaxea Nemenzo $=G$. astreata [79] 91

latifolia, Millepora 51

latifundata, Errinopora 55

Latimeria 1

latistella, Acropara 66

latistello, Poriles 75

latistellata, Moseleya 109

lotum, Flabellum 127

latum, Flobellum Studer 1878

$$
\text { = ?Monomyces rubrum [23] } 128
$$

lauensis, Galaxea 91

lawisiana, Galaxea Nemenzo = G. fascicularis [79] 91

lara, Favia 106

laxa, Goniastrea (Klunzinger 1879) = Favia laxa [65] 106

lara, Hydnophora 100

Laxa, Merulina Dana 1846

= M. ampliatal Hydnophora rigida 165] 100

laxa, Plesiastrea (Klunzinger 1879) = Favia laxa 106

laxus, Ceratotrochus 116

laysanensis, Balanophyllia 131

Leech, Medicinal 15

lehuiensis, Achatinella 22

Leiopathes 43

lelandi, Trachyphyllia Nemenzo $=T$. geaffrayi [79] 112

Lemiax 17

lenta, Antipathes 38

lenta, Leiopathes (Pourtalès 1871) = Antipathes lenta 38

lenripinna, Antipathes 38

Lepidocyathus Brook 1893 = Acrapara 65

Lepidapora 56

Lepidotheca 56

Leptastrea 107

Leptopenus 82

leptophylla, Favia 106

Lepiophylla, Polyphyllia Ehrenberg $1834=P$. talpina [44] 88

Leptopsammia 134

Leptaria 108

Leptoserir 80

Leptosmilia Milne Edwards \& Haime 1848 = Euphyllia 118

Lessani, Tropidocyathus 126

lessanii, Flabellum Michelin 1842

= Tropidocyathus lessoni [23] 126

Lelepsammia 82

leucarraphe, Achatinella 22

levicallis, Pachyseris (Dana 1846) = P. speciosa [65] 81

levidensis, Monomyces (Gardiner 1899)

$$
\text { = Rhizatrachus levidensis [23] } 129
$$

levidensis, Rhizotrochus 129

levis, Cralerastrea 77

levis, Manripara 71 levis, Montipora Quelch $=\boldsymbol{M}$. digitata [65] 70

levis, Porites Dana $=P$. cylindrica [65] 74

levistei, Lithophyllon 87

levistei, Lithophyllon Nemenzo 1971 = ?L. undulatum [44] 87

Lexingtonia 19

leytensis, Simplastrea Nemenzo

= Plesiastrea versipora 179$] 111$

lianae, Acropara 66

lianae, Acropora Nemenzo $=$ ?A. Loripes 66

Librata, Acropora Nemenzo = A. millepora [79] 67

lichen, Goniapara (Dana 1846) = Porites lichen 75

lichen, Montipora 71

lichen, Porites 75

lichtensteini, Physogyra 123

lidderdalei, Armandia $=$ Bhutanitis liddendalii 9

lidderdalii, Bhutanitis 9

lignaria, Powelliphanta = Paryphanta lignario 24

ligulata, Pocilloporo 63

lila, Achatinello 22

lilacea, Siderastrea ? = S. savignyana [65] 78

Lilli, Plesiastrea 111

lilliei, Antipathes Totton 1923 = Parantipathes lilliei 44

lilliei, Parantipathes 44

limotulus, Labyrinthacyathus 120

limar, Herpolitha 87

limar, Madrepora Esper 1797 = Herpalitha limax [44] 87

limosa, Pariles 75

linnaei, Fungia Milne Edwands \& Haime 1851

$$
=F \text {. repanda }[44] 85
$$

listeri, Acropora 66

listeri, Astreopora 69

litae, Echinopora Nemenzo $=E$. lamellosa [79] 105

Lithactinia Lesson 1831 = Polyphyllia [44] 88

Litharea Milne Edwards \& Haime 1849 = Goniopora 72

Lithomyces Philippi 1887 = Flabellum [23] 126

Lithophyllia Milne Edwards \& Haime 1857 = Scolymia 98

Lithophyllon 87

livida, Achatinella 22

livida, Distichopora 54

lizardensis, Favia 106

Lobactis Verrill $1864=$ Fungia 85

lobata, Goniopara 73

labasa, Lithaphyllon 87

lobata, Lithophyllon van der Horst 1921

= ?L. undulatum [44] 87

lobata, Podabacia van der Horst 1921

$$
\text { = Lithaphyllon undulatum [44] } 87
$$

lobata, Porites 75

lobata, Stylophara 64

lobatus, Coenocyathus Chevalier 1966

$=$ C. cylindricus [107] 116

Lobophyllia 97

Lobulata, Fungia Ortmann $1889=F$. scruposa [44] 86

Lobulata, Montipora 71

Lobulata, Montipora Bermard $1897=$ ?M. spumosa [65] 71

Lochmaeotrochus 120

lonchitis, Stylaster 59

langibrachiata, Antipathes 38

longicyathus, Acropora 66

longispina, Srichapathes 45

Laphelia 120

Lophohelia Milne Edwards \& Haime $1857=$ Lophelia 120

Lophoseris Milne Edwards \& Haime 1849 = Pavona 81

Laphasmilia Milne Edwards \& Haime 1848 = Phyllangia 90

lorata, Achatinella 22

landhowensis, Acanthastrea 95

lonicala, Acropara Nemenzo = A. Latistella [79] 66

loripes. Acropara 66 
louwince, Halomitra van der Horst 1921

$$
=H \text {. pileus }[44,79] 86
$$

lovelli, Acropora 67

lowei, Inferialabiata 55

lowekcyesei, Flabellum 127

loyae, Blastomussa 96

Iudlowi, Bhutanitis 9

Lungfish, Australian 1

Lungfish, Queensland 1

lutea, Porites 75

luckeni, Acropora 67

lukeni, Cirmipathes (Brook 1889) = Stichopathes lutkeni 45

lukkni, Stichopathes 45

Iuzonica, Acropora 67

bydius, Omithoptera croesus 10

bmani, Dasmosmilia 117

byra, Bathypathes 42

mabahithi, Caryophyllia 115

macandrewi, Flabellum 127

macassarensis, Indophyllia 96

macdonaldi, Cynoscion 3

macdonaldi, Totoaba = Cynoscion macdonaldi 3

maclurii, Endopachys 133

macrodentata, Astrangia 89

macrogastra, Errina 55

macropora, Lepidotheca 56

macrastoma, Acropara (Brook) = A. tenuis [65] 68

macrostoma, Astreopora 69

mactanensis, Montipora 71

maculata, Rhizosmilia 124

madagascariensis, Fungia Vaughan $1906=F$. scruposa 86

maderensis, Stenohelia 57

Madrepora 92

Madrepora auct. = Acropora 65

Maeandroseris 77

magellanus, Troides 13

magellanus, Troides magellanus 13

magna, Turbinaria Bernard $=T$. frondens [65] 136

magnaghii, Ceratotrochus 116

magnifica, Acropora 67

magnificum, Flabellum 127

magnistellata, Monzastrea 109

major, Conopora 53

major, Conopora Hickson \& England 1909

$$
=\text { ?C. verrucasa } 53
$$

malampaya, Monnipora 71

maldivensis, Hydnophora Gardiner $1904=H$. exesa [97] 100

maldivensis, Montipora 71

maldivensis, Pavona (Gandiner 1905) ?= P. cactus 81

maldivensis, Pavona 82

maldivensis, Stephanocoenia Gardiner 1904

= Favites pentagona [97] 107

malouinensis, Balanophyllia 131

mamillata, Stylophora 64

mammifera, Montipara Bernand = M. tuberculosa [65] 71

mammiformis, Echinopora 105

mannillosa, Plesiastrea (Klunzinger)

$$
=P \text {. versipora }[65] 111
$$

mammosa, Diploria (Dana 1846) = D. clivosa 104

manauliensis, Mantipora 71

mancaoi, Mycedium 93

mangarevensis, Acropora Vaughan $1906=$ A. clothrala 66

Manicina 108

mannarensis, Porites 75

manni, Acropora (Quelch) $=$ A. aspera [79] 65

mansfieldi, Amandia = Bhutanitis mansfieldi 9

mansfieldi, Bhuranitis 9

mansfieldi, Yunnanopapilio = Bhulanitis mansfieldi 9 manuelensis, Rhizopsammia 134

marapokensis, Troides andromache 12

marchadi, Asterosmilia 114

marchadi, Dasmosmilia Chevalier 1966

= Asterosmilia marchadi [107] 114

marchani, Paryphanta 24

marchanti, Powelliphanta = Paryphanta marchanti 24

marcus, Flabellum 127

marenzelleri, Enallopsammia 133

marenzelleri, Flabellum 127

marenzelleri, Fungiacyathus 83

marenzelleri, Srylaster 59

margaritata, Vaughanella 126

marginala, Cycloseris (Boschma 1923)

$?=$ C. costulatalenuis [44] 84

marginata, Cycloseris 84

marginala, Fungia Boschma 1923 = Cycloseris marginala 84 marigandoni, Polycyathus 124

marionensis, Alveopora 72

maritima. Favia 106

marmorea, Caryophyllia 115

mamorea, Turbinaria 136

maroccanus, Srylaster 59

marshae, Coscinaraea 76

marshae, Srylaster 59

marshallensis, Montipora 71

martensii, Truncatoflabellum 129

massawensis, Acropora (Marenzeller 1906)

$$
=\text { A. granulosa }[65] 66
$$

matthai, Anacropora 68

mathaii, Favia 106

mathaii, Porites 75

mawiensis, Trachocyathus 126

mauritiana, Pacillopora 63

mauritiensis, Goniopora (Bernard) = Poriles mauritiensis 75

mauritiensis, Porites 75

maurus, Troides helena 13

maxima, Acanthastrea 95

marima, Echinaphyllia 93

maxima, Favia 106

mayeri, Coelosiris 79

mayeri, Porites 75

mayori, Hydnophara Hoffmeister $1925=H$. rigida [97] 100

mayori, Manicina 108

moneilli, Coscinaraea 76

Meandra Oken $1815=$ Meandrina 113

meandrina, Euphyllia Dana $1846=E$. fimbriala 119

meandrina, Montipora (Ehrenberg 1834) = M. danoe [65] 70

meandrina, Pocillopora 63

Meandrina 113

meandrites, Meandrina 113

medicinalis, Hinudo 15

medioallantica, Crypthelia 53

mediterranea, Antipathes 38

medicerraneus, Polycyachus Best 1968

$$
=P \text {. muellerae [107] } 124
$$

mediterraneus, Trochocyathus 126

melicenum, Faviles (Ehrenberg) $=$ ?F. chinensis [65] 106

melicenum, Faviles 106

mrmorialis, Goreaugyra (Milne Edwards \& Haime 1849)

= Meandrina memorialis 113

memorialis, Meandrina (Wells 1974)) $=$ ?M. meandrites 113

memorialis, Meandrina 113

meridiana, Acropora Nemenzo = A. brueggemanni [79] 65

meridionalis, Omithoptera 10

meridionalis, Omithoptera meridionalis 10

meridionalis, Omithoptera paradisea $=0$. meridionalis 10

meridionalis, Schoenbergia $=$ Omithoptera meridionalis 10

meridionalis, Trochocyathus 126 
merleti, Blastamussa 96

Merulina 100

mesenterina, Turbinaria 136

messum, Flabellum 127

Metastrea Milne Edwarda \& Haime 1857 = Favites 106

Mexican Red-kneed Tarantula 14

mexicana, Cycloseris Durham $1947=$ Diaseris distona 85

michelini, Astrangia Milne Edwarda \& Haime 1848

$$
\text { = A. poculata } 89
$$

michelini, Heieropsammia Milne Edwards \& Haime 1848

$$
=H \text {. cochlea [65] } 133
$$

michelinii, Stephanocoenia 6

michelinii, Stephanocoenia Milne Edwarda \& Haime 1848 $=S$. inversepta 6]

Micrabacia 82

micrantha, Tubastraea 135

micranthus, Dendrophyllia 132

microclados, Acropora 67

microconas, Hydnophora 100

Microcyathus Döderlein 1913 = Hoplangia 120

microphthalma, Acropora 67

microphthalma, Cyphastrea 103

micropama, Crypthelia 53

micropoma, Sporadopora 57

microstriatus, Stylaster 59

microstylus, Lepidopora 56

Microtrochus Tenison-Woods $1880=$ ?Placotrochus [23] 128

milesii, Distichopora Quelch $1885=D$. gracilis [14] 54

millepora, Acropora 67

millepora, Mantipora 71

Millepora 51

milleri, Stylaster 59

mimosella, Antipathes Lamarck $1816=$ A. ulex 40

miniata, Allopora Pourtalès 1868 = Stylaster miniatus [18] 59

miniatus, Stylaster 59

minicoiensis, Porites 75

minima, Stenahelia 57

minimus, Discorrochus Yabe \& Eguchi 1937

= Peponocyathus folliculus [23] 122

minor, Anripathella Brook 1889 = Antipathes minor 38

minor, Anripathes 38

minor, Gardineria 128

minor, Goniopora 73

minor, Pavona 82

minor, Tethocyathus 125

minos, Troides 13

minos, Troides helena $=T$. minos 13

minus, Flabellum Duncan $1878=F$. alabasirum [107] 126

minuscula, Dendrophyllia 132

minuta, Acanthastrea 95

minuta, Cyphastrea Nemenzo \& Ferraris

= C. microphthalma [79] 103

minuta, Pavona 82

minuta, Rhizopsammia 134

minutus, Deleocyathus 118

minutus, Deltocyathus Gardiner \& Waugh 1938

= ?Peponocyathus australiensis [23] 122

miakensis, Omithoptera priamus 10

mirabilis, Acropara 67

mirabilis, Barabattoia 101

mirabilis, Madracis 62

miranda, Troides 13

miranda, Troides miranda 13

misoolana, Omithoptera tithonus 10

modesta, Barabattoia Nemenzo $=$ B. amicorum [79] 101

madumanensis, Pocillopora Vaughan $1907=$ P. eydouxi 63

mokai, Lithophyllon 87

mollis, Montipora 71

mollis, Turbinaria 136 molokensis, Paracyathws 122

molokensis, Pocillopora 63

moluccensis, Fungia 85

monasteriata, Montipora 71

monile, Coscinaraea 76

moniljformis, Millepora Dana $=M$. alcicomis 51

Monocarya Lonsdale $1850=$ Parasmilia 122

Monomyces 128

Monopora Dana 1848 = Monripora 69

Montastrea 109

monsereyensis, Paracyathus 122

montereyensis, Pobymyces 129

Monticularia Lamarck 1816 = Hydnophora 99

monticulosa, Acropara 67

manticulosa, Pachyseris (Verrill 1866) $=$ P. rugosa 81

monticulosa, Porites Dana $1846=P$. rus [65] 75

Montigyra 121

Montipora 69

mopa, Troides helena 13

mordax, Poriles 75

mordax, Stylophora (Dana 1846) $=S$. pistillata [59] 64

moretonensis, Astreopora 69

moretonensis, Heteropsammia 133

mortenseni, Alveapora Crossland = A. allingi [65] 72

morienseni, Sparadopora 57

moriensi, Flabellum 127

moschylus, Troides helena 13

Moseleya 109

moseleyanus, Stephanocyathus 125

moseleyanus, Stylaster 59

moseleyi, Adelopara 52

moseleyi, Crypthelia Hickson \& England $=C$. affinis [14] 53

moseleyi, Deltocyathus 118

moseleyi, Errina Ridley $1881=E$. antarctica [14] 54

moseleyi, Flabellum 127

mosekyi, Stylaster (Dall 1884) $=S$. verrillii [14] 59

motuporensis, Padabacia 87

mouchezii, Coenocyathus Lacaze-Duthiers 1897

= Phyllangia mouchezii [107] 90

mouchezii, Phyllangia 90

mucronata, Porites 75

muellerae, Polycyathus 124

multiacula, Acropora 67

multicaulis, Acropora)Brook ) = A. valenciennesi [65] 68

multiflora, Isophyllia 97

mulniformis. Acropora (Ortmann) = A. formosa [65] 66

multilobata, Goniastrea Quelch $1886=G$. pectinata [97] 107

multilobatus, Thrypticotrochus 125

mulripapillosa, Montipora 71

multiplex, Stylaster 59

multipunctata, Montastrea 109

multiramosa, Acropora Nemenzo $=$ A. austera [79] 65

murrayensis, Acropora Vaughan $1918=$ A. Loripes 66

murrayensis, Porikes 75

murrayi, Millepara 51

muscasa, Goniopara 73

musculosa, Cirrhipathes 42

musica, Tubipora 60

musorsiomica, Gardineria 128

Mussa 97

Mussel, Long Solid 19

Muasel, Orange-footed Pimpleback 20

Mussel, Ozark Broken-ray 19

Mussel, Sandback Pockethook 19

Mussel, Westem Fanshell 18

Mussel, White Catspaw 18

Mussismilia 98

mustelina, Achatinella 22

Mycedia Dana 1848 = Agaricia 79 
Mycedium 93

Mycetophylia 98

myceroseroides, Leptoseris 80

mycoides, Cycloseris Alcock $1893=C$. sinensis [44] 84

myriaster, Madracis 62

myriophthalma, Astreopora 69

myriophylla, Antipathes 38

myrmidonensis, Porites 75

naias, Troides haliphron 12

nana, Acropora 67

nana, Cirrhipathes 42

nana, Stylophora Nemenzo $=S$. pistillata [79] 64

nanneca, Errinopora 55

naomiae, Alveopora Nemenzo $=A$. verrilliana [79] 72

Napopara Quelch $1886=$ Porites 73

nascomatus, Tropidocyathus 126

nascomatus, Tropidocyathus Gardiner \& Waugh 1938

$$
=? T \text {. pileus [23] } 126
$$

nasiformis, Congregopora 52

nasuia, Acropara 67

natans, Colpophyllia 103

natunensis, Trogonoptera brookiana 12

neapolitanus, Microcyathus Döderlein 1913

= Hoplangia durotrix [107] 120

neglecta, Stephanophyllia 82

negrensis, Galaxea Nemenzo = G. astreata [79] 91

negrosensis, Parites 75

Nemenzophyllia 121

Nemenzophyllia Hodgson \& Ross 1981

$$
?=\text { Plerogyra 175.79] } 123
$$

Neoceratodus 1

Neohelia 92

neomiranda, Troides miranda 13

Neoporites Duchassaing \& Michelotti $1860=$ Porites 73

neoris, Troides helena 13

nephereus, Troides $=7$. rhadamantus 13

nereides, Troides helena 13

nereis, Traides helena 13

niasicus, Troides amphrysus 12

nickliniana, Megalonaias = Unio nickliniana 20

nickliniana, Unio 20

nierstraszi, Psammocora 78

nigra, Goniopora Pillai $=$ G. suchburyi $[65] 73$

nigrescens, Dendrophyllia 132

nigrescens, Porites 75

niinoi, Crispatotrochus 117

niinoi, Rhizotrochus 129

nilanduensis, Antipathes 38

niphada, Rhombopsammia 82

nishihirai, Echinophyllia 93

nitens, Stephanotrochus Gravier 1920

= Stephanocyathus crassus [107] 125

nitida, Distichopora 54

nitida, Millepora 51

nirida, Turbinaria Nemenzo $=T$. stellulata [79] 136

nobile, Desmophyllum Verrill 1885

= Javania cailleti $[23,107] 128$

nabile, Flabellum 127

nobile, Flabellum Holdsworth 1862

= ?Monomyces rubrum [23] 128

nobile, Javania (Verrill 1885) $=J$. cailleti 128

nobilis, Acropora 67

nobilis, Leptoseris Ma $1959=$ L. cucullata |35] 80

nobilis, Pocillopora Verrill $1864=P$. woodjonesi 63

nobilis, Stephanocyathus 125

nobilis, Stylaster 59

nobilis, Symphyllia (Dana 1846) = S. recia [65] 99

nobilis, Symphyllia 99 nodifera, Porites 75

nodosa, Montipora 71

nodulosa, Montipora Nemenzo $=M$. malampaya [79] 71

nodulosa, Porices 75

nomaenis, Antillia Yabe \& Sugiyama

$$
\text { = Cynarina lacrymlis [2] } 96
$$

nomis, Troides $=T$. minos 13

Nomlandia 121

norfolkensis, Goniopora 73

norvegicus, Stylaster 59

Notocyathus 121

Notophyllia 134

Nototrochus Duncan $1885=$ Notocyathus [23] 121

noumeae, Cantharellus Hoeksema \& Best 1984

$=$ Cycloseris noumeae 94

noumeae, Cycloseris 84

novaehibemiae, Polyphyllia 88

novaeze (a)landiae, Errina 55

nutrix, Blastotrochus 126

oahense, Endopachys Vaughan $1907=$ E. grayi 133

oahensis, Dendrophyllia 132

oahensis, Fungia Döderlein $1801=F$. scutaria $[44] 86$

oahensis, Trochocyathus 126

obiana, Omithoptera $=O$. aesacus 9

abiensis, Omithoptera $=0$. aesacus 9

obliquus, Conopora (Studer 1878) $=$ C. laevis [14] 53

obliquata, Plagiola 18

oblongomaculatus, Troides 13

ablongomaculatus, Troides oblongomaculalus 13

oblongomaculatus, Troides helena $=T$. oblongomaculatius 13

abscura, Acropora (Brook) $=$ A. humilis $[65] 66$

ablusangula, Psammocora 78

obusata, Pavona (Quelch) $=$ P. venosa [65] 82

occidentalis, Stichopathes (Gray ?) $=S$. gracilis 4 .

ocellata, Acropora (Klunzinger 1879) = ?A. humilis [65] 66

ocellata, Acropora 67

ocellata, Alveopora 72

acellata, Astreopora 69

ocellina, Cyphastrea 103

ochracea, Distichopora Quelch $1884=D$. nirida [14] 54

achraceus, Stylaster (Quelch 1884) $=$ S. nabilis [14] 59

actoformis, Alveopora 72

actopali, Caryaphyllia 115

octoptera, Seriatopora Ehrenberg $=S$. caliendrum 64

oculata, Amphelia 90

oculata, Madrepora Linnaeus $1758=$ ?M. galapagensis 92

oculeus, Lochmaeotrochus 120

Oculina 92

Odonthocyathus Brook 1893 = Acropora 65

Odontocyathus 121

officinalis, Hirudo $=H$. medicinalis 15

olinawensis, Calalaphyllia 116

okinawensis, Porites 75

oldraydi, Dendrophyllia 132

oligocyathus, Acropora 67

ongulense, Flabellum 127

Orbicella Dana $1846=$ Moniastrea 109

orbicularis, Acropora (Brook) = A. clathrata [65] 66

orbiculata, Lampsilis orbiculata 19

orientalis, Antillia Gerth 1921

= Trachyphyllia geofforyi [2] 112

orientalis, Dellocyathus Duncan 1876

= Peponocyathus australiensis [23] 122

orientalis, Mantipora 71

orientalis, Notocyathus (Duncan 1876) = Peponocyathus ausiraliensis 123] 122

orientalis, Paradeltocyathus (Duncsn 1876) = Peponocyathus australiensis [23] 122 
orientalis, Peponocyathus (Duncan 1876)

$=P$. australiensis [23] 122

orientalis, Peponocyathus 122

orientis, Troides helena 13

omata, Systemopore 59

omatus, Dellocyathus 118

Ornithoptera 9, 10

orpheensis, Echinophyllia 93

Orthonymus 20

Oryzotrochus 121

osbumi, Balanophyllia Durham \& Bamard 1952

= B. galapogensis [25] 131

ostreaeformis, Coscinaraea van der Horst 1922

$=C$. monile [65] 76

Oulangia 90

Oulastrea 109

Oulophyllia 110

ovalis, Astreopora 69

owenii, Flabellum Milne Edwards \& Haime 1848 = Truncatoflabellum stokesi [23] 129

Oxyphyllia Yabe \& Eguchi 1935 = Echinophyllia 93

Oxypora 94

axyrhynchus, Acipenser 1

Orysmilia 121

pachypoma, Cahypiopora 52

pachypoma, Crypiahelia Hickson \& Enga!

= Pseudocrypthelia pachypoma [15] 57

pachypoma, Pseudocrypthelia 57

Pachypsammia Verrill 1866 = Coenopsammia 132

pachysepta, Lobophyllia 97

Pachyseris $\mathbf{8 0}$

pacifica, Anthemiphyllia 113

pacifica, Cladocora 102

pacificus, Echinopora 105

paeonia, Pectinia 94

pagoensis, Acropora 67

Palauastrea 62

palauensis, Favites (Yabe, Sugiyama \& Eguchi 1936)

= Goniastrea palauensis 107

palauensis, Favites 106

palauensis, Goniastrea 107

Palauphyllia Yabe Sugiyama \& Eguchi 1936

$$
\text { = Lobophyllia } 97
$$

palifera, Acropora 67

palifera, Balanophyllia 131

palifera, Polycyathus 124

paliferus, Fungiacyathus 83

paliferus, Stephanocyathus 125

pallaryi, Hoplangia Joubin 1930

= Cladocora caespitosa [107] 102

pallens, Oculina Ehrenberg $1834=0$. diffisa 92

pallens, Troides haliphron 12

pallida, Favia 106

pallida, Phyllangia Klunzinger 1879

= Polycyathus pallidus [107] 124

pallidus, Polycyathus 124

palmala, Acropora 67

palmala, Montipora 71

palmata, Porites 75

palmata, Stylophora Blainville $1830=$ S. pistillata [65] 64

palmensis, Goniopora 73

palmerae, Acropora 67

palmerae, Acropora Wells $1954=$ ?A. rabusta [65] 67

paloensis, Rhizotrachus Yabe \& Eguchi 1942

$$
=R \text {. typus [23] } 129
$$

panamensis, Anripathes 38

panamensis, Leplaseris Durham \& Bamard 1952

$=L$ papyracea $[35] 80$ panamensis, Poriles Vaughan $1919=P$. woylandi 75

panda, Caryophyllia 115

pandanus, Favia (Dana 1846) $=$ F. speciosa 106

pandoraensis, Goniopora 73

Pangasianadon 2

paniculasa, Acropora 67

paniculata, Antipathes 38

paniculata, Arachnopathes Duchassaing \& Michelotti 1864 $=$ ?Antipathes atlantica 35

Papilio 11

papillosa, Crypthelia 53

papillosa, Fungia Verrill $1866=F$. fungites [44] 85

papillosa, Montipora 71

popillosa, Stichopathes 46

papillosa, Stylantheca 58

papillosa, Stylaster 59

papuensis, Phyllangia 90

papuensis, Stylaster 59

papuensis, Troides oblongomaculatus 13

Papustyla 23

papyracea, Achatinella 22

papyracea, Folioseris Rehberg 1892

$=$ Leptoseris gardineri [35] 80

papyracea, Leptoseris 80

papyracea, Pavonia Dana 1846

= Leptoseris papyracea [35] 80

paraancora, Euphyllia 119

Paraclavarina 100

Paraconotrochus 121

Paracyathus 122

Paradeliocyathus Yabe \& Eguchi 1937

= Peponocyathus [23] 122

Paradichocoenia Alloiteau 1957 = Dichocoenia 113

paradisea, Omithoptera 10

paradisea, Omithoptera paradisea 10

paradivisa, Euphyllia 119

paradoxa, Gardineria 128

paradoxus, Caryophyllia 115

Paraerrina 56

paraglabrescens, Euphyllia 119

Parahalomitra Wells $1937=$ Sandalolitha [44] 88

Parantipathes 44

Parascolymia Wells $1963=$ Scolymia 98

Parasimplastrea 110

parasiticus, Dunocyathus 118

Parasmilia 122

Parasmilia Milne Edwards \& Haime 1848 = ?Coenosmilia/Dasmosmilia $116 / 117$

Parastrea Milne Edwands \& Haime 1848 = Favia 105

Paratrochocyathus Alloiteau 1958 = Trochocyathus 126

parilis, Acropora 67

paripavoninum, Flabellum Alcock 1894

= Truncatoflabellum paripavoninum [23] 129

paripavoninum, Truncasaflabellum 129

Pamassius 11

parseplatus, Platytrachus 123

parvastella, Goniopora 73

parvicella, Favites Nemenzo 1959

$=F$. pentagona $[79,97] 107$

parvistella, Acropora 67

parvistellasa, Porites 75

parula, Caryophyllia 115

Paryphania 23, 24

paschalensis, Leptoseris Wells $1972=$ L. solida [35] 80

paschalensis, Porites 75

patagonica, Oculina 92

patella, Fungia (Ellis \& Solander 1786) $=F$. fungites [44] 85

patellaris, Fungia (Lamarck 1801) $=F$. fungites [44] 85 
patelliformis, Cycloseris (Boschma 1923)

$?=$ C. cyclolites/Diaseris fragilis [44] 85

patelliformis, Cycloseris 84

patelliformis, Fungia Boschma 1923

= Cycloseris patelliformis 84

patens, Flabellum 127

patera, Anthemiphyllia 113

Paterocyathus Duchassaing \& Micheloti 1860

$$
\text { = Caryophyllia } 114
$$

patula, Acropora (Brook) = A. latistello [65] 66 patula, Bathypaches 42

patula, Diploastrea (Dana 1846) $=$ D. heliopora 104

patula, Echinophyllia 93

parula, Monsipora 71

parula, Montipora Verrill $1869=2 M$. hispida [65] 71

patula, Physophyllia Hodgson \& Ross 1981

= Echinophyllia patula 93

patula, Turbinaria 136

paucipalata, Caryophyllia 115

paucisepra, Favia Chevalier $1972=F$. pallida [98] 106

paucisepta, Galaxea 91

pauciseptata, Caryophyllia 115

pauciseptata, Conopora Broch $1951=C$. verrucasa $[14] 53$

pauciseptata, Stenohelia 57

paucispina, Cirmipathes Brook 1889

= Stichopathes paucispina 46

paucispina, Stichopathes 46

paumotensis, Fungia 85

paupera, Montipora 71

pauroclema, Antipathes 38

Pavana 81

Pavania Lamarck 1816 = Pavana 81

pavoninum, Flabellum 127

paxilligera, Acropora (Dana 1846) $=$ A. humilis [65] 66

Pearly Mussel, Alabama Lamp 19

Pearly Mussel, Appalachian Monkey-face 20

Pearly Mussel, Bindwing 17

Pearly Mussel, Brown-blossom 19

Pearly Mussel, Clubshell 20

Pearly Mussel, Cumberland Bean 20

Pearly Mussel, Cumberland Monkey-face 20

Pearly Mussel, Curtis' 18

Pearly Mussel, Dromedary 18

Pearly Mussel, Edible 18

Pearly Mussel, Fat Pocketbook 20

Pearly Mussel, Fitre-rayed Pigioe 19

Pearly Mussel, Green-blossom 18

Pearly Mussel, Higgins' Eye 19

Pearly Mussel, Nicklin's 20

Pearly Mussel, Ozark Lamp 19

Pearly Mussel, Pale Lilliput 20

Pearly Mussel, Pink Mucket 19

Pearly Mussel, Plain Pocketbook 19

Pearly Mussel, Rough Pigtoe 20

Pearly Mussel, Sampson's 18

Pearly Mussel, Shiny Pigtoe 19

Pearly Mussel, Slab-sided 19

Pearly Mussel, Tampico 20

Pearly Mussel, Tan-blossom 18

Pearly Mussel, Tubercled-blossom 18

Pearly Muasel, Turgid-blossom 18

Pearly Mussel, White Warty-back 19

Pearly Mussel, Yellow-blossom 18

pectinata, Actinastrea 61

pectinata, Antipathes 38

pectinata, Fungia Ehrenberg 1834

$$
=\text { Cuenactis echinata [44] } 83
$$

pectinaka, Goniastrea 107

pectinia, Acropora (Brook) = A. hyacinthus [65] 66
Pectinia 94

pedala, Antipathes 38

pedata, Aphanipathes (Gray 1857) = Antipathes pedata 38

pedata, Savagliopsis (Gray 1857) = Antipathes pedata 38

pedersenii, Astropsammia 130

pedersenii, Astropsammia Verrill 1869

= ?Tubasiraea coccinea [25] 135

pedersenii, Tubastraca (Verrill 1869) $=T$. coccinea 135

pedicellatus, Placotrochus 128

pedunculata, Goniapara 73

peircei, Crypthelia 53

pelsata, Turbinaria 136

peliformis, Montipora 71

pendulus, Goniopora 73

peninsulae, Troides cuneifer 12

pennacea, Antipathes 38

pennacea, Aphanipathes (Pallas 1766)

$=$ Anripathes pennacea 38

pentagona, Favites 107

Peponocyathus 122

perculia, Caryophyllia i1s

peresi, Favites 107

perexigua, ldiotrochus 120

perforata, Montipara 71

perobliqua. Dysnomia sulcala

= Epioblasma sulcata perobliqua 18

perabliqua, Epiablasma obliquata $=E$. sulcala perabliqua 18

perobliqua, Epiablasma sulcata 18

perabliqua, Plagiala sulcaia

= Epiablasma sulcata perobliqua 18

periusa, Lophelia 120

periusa, Madrepara Linnseus 1758

$=$ Lophelia pertusa $[107] 120$

petrograpta, Stylantheca 58

petrograpta, Stylaster (Fisher 1938) = ?Stylantheca 58

petterdi, Thrypticotrachus 125

penerdi, Trochocyathus Dennant 1906

= Thrypticotrachus petterdi 125

Phacelocyathus 122

phaeazona, Achutinella 22

Phalangopara 56

pharaonis, Acropara 67

pharaonis, Acropora Pillai $1971=$ A. teres [59] 68

pharensis, Astrocoenia Heller 1868

= Madracis pharensis [107] 62

pharensis, Madracis 62

philippinensis, Acropara 67

philippinensis, Endopsammia 133

philippinensis, Gardineria 128

philippinensis. Halomirra Studer $=H$. pileus $[65,79] 86$

philippinensis, Podabacia Studer 1901

= Halomitra pileus [44] 86

phrygia, Leptoria 108

phrygia, Platygyra (Ellis \& Solander 1786)

$$
\text { = Leptoria phrygia } 108
$$

Phyllangia 90

Phyllastrea Dana $1846=$ Mycedium 93

Phyllopora Ehrenberg 1834 = Astreopora 69

Pr.yllopora Tenison Woods $1879=$ Stylophara 64

Phymastrea Milne Edwards \& Haime 1848 = Favia 105

Physogyra 122

Physophyllia 94

picea, Antipathes Pourtales $1880=$ A. hina 37

picleri, Euphyllia 119

pilejformis, Polyphyllia Dana 1846

$=P$. novaehiberniae [44] 88

pileus, Halomitra 86

pileus, Madrepora Linnaeus 1758 = Halomitra pileus $[44] 86$ 
pileus, Trochocyathus Alcock 1902

= Tropidocyathus pileus [23] 126

pileus, Tropidocyathus 126

pilosa, Hydnophora 100

pinguis, Acropora Wells $1950=$ A. robusta $[65] 67$

pini, Platygyra 111

Pink Conch 24

pinnarifida, Antipathes Lamouroux ? = ?A. myriophylla 38

Pirarucu 2

pistillata, Stylophora 64

pistor, Troides haliphron 12

Pla Buk 2

Pla Eesok 2

Placotrochides 128

Placotrochus 128

placunaria, Fungia KJunzinger $1879=$ F. scutaria [44] 86 Plagiola 18

plana, Acropora Nemenzo = A. tenuis [79] 68

plana, Antipathes 38

plana, Caulastrea 102

plana, Caulastrea Hodgson \& Ross 1982

= ?C. tumida [79] 102

plana, Fungia (Studer) $=F$. concinna [65] 85

planilamellata, Caryophyllia 115

planipora, Psammocora Milne Edwards \& Haime $=P$. contigua $[65] 77$

planiuscula, Montipora (Dana 1846) $=M$. vernucosa 72

planocella, Porites Nemenzo $=$ P. cylindrica [79] 74

plantagenista, Antipathes 39

plantagenista, Aphanipathes Forster Cooper 1903 = Antipathes plantagenista 39

plantaginea, Acropora (Dana 1846) = A. secale $[65] 67$

planulata, Gardineroseris 79

planulata, Goniopora 73

planulata, Pavona (Dana 1846)

= Gardineroseris planulata $[65179$

planus, Flabellum 127

plateformis, Montipora Nemenzo $=$ M. hispida [79] 71

plateni, Troides 13

plateni, Troides rhadamantus $=T$. plateni 13

plato, Troides 13

plato, Troides haliphron $=T$. plato 13

platycaulus, Bathypathes 42

platycyathus, Acropora 67

Platycyathus 123

Platygyra 110

platyphylla, Millepora 51

plarypoma, Crypthelia 53

playpus, Stephanocyathus 125

Plarytrochus 123

plena, Quadrula condata $=$ Pleurabema plenum 20

plenum, Pleurobema 20

Plerogyra 123

Plesiastrea 111

Plesioseris 77

Plethobasus 19

Pleuractis Verrill $1864=$ Fungia 85

Pleurobema 20

Pleurocyothus Moseley $1880=$ ? Ceratotrochus 116

plicasa, Calalaphyllia (Milne Edwards \& Haime 1848)

$$
\text { = C. jardinei [65] } 116
$$

plicara, Millepora 51

plicata, Pectinia Nemenzo = P. alcicomis [79] 94

plicala, Pocillopora 63

pliciseptus, Fungiocyathus 83

pliculosa, Fungia Studer $1877=F$. fungites $[44] 85$

Pliobothrus 57

pluma, Antipathes 39

plumosa, Cladopathes 43
Pocillopora 63

poculata, Astrangia 89

Podabacia 87

polarensis, Favites 107

politum, Flabellum 127

pollicala, Pavona Wells $1954=P$. maldivensis 82

Polyastra Ehrenberg 1834 = Pavona 81

Polycyathus 90,123

potyformis, Goniopora 73

pobygona, Caryophyllia Pourales 115

polymorpha, Acropora 67

polymorphus, Srylaster 59

Polymyces 129

Patyodon 1

potyorchis, Stylaster 59

Pobyphyllia 88

polypoma, Crypthelia 53

Polystachys Brook 1893 = Acropora 65

polystichopora, Lepidopara 56

polysıma, Acropora 67

pompeus, Troides $=T$. minos 13

ponderosa, Acropora Nemenzo = A. robusta [79] 67

ponderosa, Agaricia Gardiner 1905

= Gardineroseris planulata [65] 79

ponderosa, Agaricio var. minikoiensis (Gardituer 1905)

= Gardineroseris planulata [65] 79

ponderosa, Gardineria (Gardiner)

= Gardineroseris planulata [65] 79

ponderosa, Gardineroseris Scheer \& Pillai 1974

$=$ G. planulata [59] 79

ponderosa, Pavona (Gardiner)

= Gardineroseris planulata [65] 79

porcellana, Madrepora (Moseley 1881)

= Neohelia porcellana 92

porcellana, Neohelia 92

porcellana, Turbinaria 136

porcellanus, Hippopus 16

porifera, Errinopora 55

porites, Porites 75

Porites 73

poritiformis, Montipora 71

porosa, Domoseris Quelch $1886=$ Leptoseris solida [35] 80

porosa, Leptoseris (Quelch) $=$ L. solida [65] 80

porosa, Pocillopora 63

porphyra, Sylaniheca 58

porphyreus, Paracyathus 122

portoricensis, Goniopora Vaughan 1919

$$
=\text { Porites portoricensis } 75
$$

portoricensis, Orysmilia 121

portoricensis, Porites 75

poseidon, Omithoptera priamus 10

Potamilus 20

pourialesi, Deltocyathus 118

pourtalesi, Endopsammia 133

pourtalesi, Lepidotheca 56

pourtalesi, Srichopathes Brook $1889=$ S. gracilis 45

pouralesi, Thecopsommia Durtam \& Bamard 1952 = Endopsammia pourtalesi [25] 133

pourtalesii, Errinopora 55

Pourialocyathus 130

Pourtalosmilia 124

praecipua, Dendrophyllia 132

praetona, Pavona (Dana 1846) $=P$. cactus [65] 81

prationum, Troides 13

prava, Montipora Nemenzo $=M$. cactus [79] 70

Premocyathus 124

prescillae, Seriatopora Nemenzo $=S$. caliendrum [79] 64

pretiosa, Pavonia Bassett-Smith 1890

$$
\text { = Leptoseris papyracea [35] } 80
$$


priamus, Omithoptera 10

priamus, Omithoptera priamus 10

Prionastrea Milne Edwards \& Haime 1848 = Faviles 106

Probarbus 2

procumbens, Acropora (Brook) = A. echinata $[65] 66$ pracus, Omithaptera galiath 10

producta, Patyphyllia Folkeson $1919=$ P. Lalpina [44] 88

proechinata, Fungia Döderlein $1901=F$. paumotensis [44] 85

profunda, Astreopora Verrill $1872=$ A. myriophthalma 67

profunda, Caryaphyllia 115

profunda, Distichopora 54

profunda, Enallopsammia 133

profunda, Madracis 62

profunda, Montipara 71

profiunda, Oculina 92

profiunda, Stenohelia 57

profundacella, Psammocara 78

profundiparus, Stylaster 59

profundum, Flabellum Milne Edwards \& Haime 1848 = Truncatoflabellum prafundum [23] 129

prafundum, Truncaiaflabellum 129

profundus, Paracyathus 122

profiundus, Porites 75

profundus, Stylaster 59

profusa, Acropora 67

proficsa, Acropara Nemenzo = ?A. florida 66

prolifer, Ceratocyathus Pourtalès 1871

= Asterasmilia prolifera [107] 114

prolifera, Acrapora 67

prolifera, Asterosmilia 114

prolifera, Laphelia (Pallas 1766) = L. pertusa [107] 120

prolifera, Madrepora Pallas 1766

= Laphelia pertusa [107] 120

prolifera, Mantipara Brüggemann $=M$. foliosa [65] 70

prolixa, Acropora (Verrill 1866) $=A$. carduus 65

prominens, Acrapara Nemenzo $=$ A. palifera $[79] 67$

prapinqua, Cirrhipathes Brook $1889=$ C. anguina 42

prapinquus, Troides helena 13

prostrata, Acropara (Dana 1846) = ?A. millepora 67

prostrala, Acropara 67

prastrata, Stylophora Klunzinger $=S$. pistillata [65] 64

Protalabophyllia Yabe \& Sugiyama 1931 = Cynarina 96

Protamussa Matthai $1928=$ Mussismilia 98

pravidentiae, Distichopora 54

pruinasa, Acropora 67

pruinosa, Leptastrea 108

pruvoti, Leplopsammia 134

Psammacara 77

Psammoseris 134

pseudoalabastra, Javania 128

Pseudocolumnastrea Yabe \& Sugiyama 1933 = Pavona 81

Pseudocrypthelia 57

Pseudocyathaceras 124

pseudodichotoma, Antipathes 39

Pseudosiderastrea 78

pseudostephanus, Fungiacyathus 83

pseudothyron, Adelapara 52

Pteropathes Brook $1889=$ Antipathes 35

pudica, Crypthelia 53

puernogalerae, Anacropora 68

pukoensis, Parites 75

pulchella, Diaseris Verrill $1866=$ D. distoria 85

pulchellus, Paracyaikus 122

pulcher, Stylaster 59

pulcherrima, Achatinello 22

pulcherrima, Mantipora Bernard $1897=$ M. foliosa [65] 70

pulcherrima, Papuina = Papustyla pulcherrima 23

pulcherrima, Papustyla 23

pulcherrima, Turbinaria 136 pulchra, Acropara 67

pulchra, Leplastrea (Dana 1846) $=$ L. purpurea 108

pulchra, Rhizopsammia 134

pulvinatum, Cheiloporidion 52

pulvinula, Goniapora 73

pulvinula, Goniopora Wells $1954=G$. djiboutiensis [65] 73

pumila, Acropora 67

pumila, Millepora 51

punctata, Antipathes 39

punctata, Montipora Bemard $1892=$ M. hispida [65] 71

punctata, Porites 75

punctata, Stylaraea 76

punctatus, Stylaster 59

punctifera, Astreapara 69

punctifera, Astreopara Bemand = ?A. listeri 69

pupukanioe, Achatinella 22

Pupurangi 23

purpuratus, Stylaster 59

purpurea, Leplastrea 108

purpurea, Poriles 75

pusilla, Enallopsammia 133

pusillum, Truncataflabellum 129

pusillus, Fungiacyathus 83

pustulosa, Acropora (Milne Edwards \& Haime) =A. pharaonis [65] 67

puteolina, Favia Dana $1846=F$. speciosa 106

putnami, Favia (Verrill 1872) $=F$. pallida 106

pygmaea, Manomyces 128

pygamaea, Caryaphyllia Risso 1826

= Manamyces pygmaea $[107] 128$

pyramidalis, Acropora (Klunzinger) $=$ A. humilis [65] 66

Pyrophyllia Hickson $1910=$ Guynia [23] 130

quadragenaria, Caryophyllia 115

quadribrachiala, Bathypathes 42

Quadrula 19,20

quarylei, Cyathaceras Dutham 1947

= Labyrinthocyathus quaylei [25] 120

quaylei, Labyrinthocyathus 120

Queen Conch 24

queenslandiae, Leptopsammia 134

quelchi, Acrapora (Brook) = A. nasuta [65] 67

quoyi, Goniastrea Milne Edwards \& Haime 1850

= G. pectinata $[97] 107$

radians, Antipathes Lamarck $1816=$ A. dichotoma 36

radians, Siderastrea 78

radians, Symphyllia 99

radiara, Coenopsammia 132

radiata, Trachyphyllia (Pichon 1980)

= Wellsaphyllia radiata 112

radiaza, Wellsaphyllia 112

radiata, Wellsophyllia Pichon 1980

= ?Trachyphyllia geoffrayi [79] 112

radiatus, Monomyces (Dennant 1904)

= Rhizotrochus radiatus [23] 129

radiatus, Rhizotrochus 129

radicalis, Turbinaria 136

ralphae, Sphenotrochus 125

rambleri, Acropora 67

ramea, Dendrophyllia 132

ramiculosa, Acropora 67

ramasa, Crypthelia 53

ramosa, Hillopathes 43

ramosa, Hydnophora Nemenzo $1959=H$. grandis [79] 100

ramosa, Lepidotheca 56

ramosa, Millepara 51

ramosa, Montipora Bernard $=\boldsymbol{M}$. diginaka 70

ramosa, Palawastrea 62 
ramasa, Pavonia Bsssett-Smith 1890

$=$ Leptaseris papyracea [35] 80

ramosus, Stylaster 59

rangiana, Dysnomia tonulosa

= Epioblasma tarulosa rangiana 18

rangiana, Epiablasma corulosa 18

rangiana, Plagiola torulosa

= Epiablasma tonulosa rangiana 18

rarisepta, Favia 106

rathbuni, Astrangia 89

raukawaensis, Flabellum 127

rawsonii, Trochocyathus 126

recidivus, Aulocyathus 114

reclinata, Acropora Nemenzo $=A$, palifera $[79] 67$

recta, Notaphyllia 134

recta, Symphyllia (Dana 1846) = ?S. nobilis [59] 99

recta, Symphyllia 99

recta, Taxipathes 46

recumbens, Acropora (Brook) = A. hyacinthus [65] 66

recurvatus, Tethocyathus 125

rediviva, Archohelia 91

rediviva, Balanophyllia 131

reesei, Desmophyllum 118

reesi, Mycelaphyllia 98

reeviana, Villosa 19

regalis, Menulina Dans $1846=\boldsymbol{M}$. ampliata 100

regia, Balanophyllia 131

reginae, Omithoptera victoriae 11

regis, Omithoptera victoriae 11

regularis, Antipathes (Forster Cooper 1909)

$$
\text { = Srichopathes regularis } 46
$$

regularis, Leptaseris (Quelch) $=$ L. scabra [65] 80

regularis, Phalangopora 56

regularis, Stichopathes 46

reniformis, Montipora Nemenzo 1967 $=M$. peltiformis [79] 71

renifarmis, Turbinaria 136

repanda, Fungia 85

repens, Coenosmilia Chevalier 1966

$$
\text { = Pourtalosmilia anthophyllites [107] } 124
$$

repens, Pavona 82

reptans, Anacropora 68

reptans, Ganiopara 73

reticulata, Acropora $($ Brook $)=A$. cytherea [65] 66

reticulata, Anacropora 68

reticulata, Antipathella (Esper 1788)

$$
\text { = Antipathes reticulata } 39
$$

reticulata, Antipathes 39

reticulata, Aphanipathes 41

reticulata, Calyptopara 52

reticulata, Errina 55

reticulasa, Porites 75

reticulum, Errinopsis 55

retiformis, Goniastrea 107

retipara, Alveapora 72

retusa, Acropora 67

renusa, Alveopara 72

Reussastrea d' Achiardi 1875 = Pavona 81

Rhabdocyathus Brook 1893 = Acropora 65

hadamantus, Troides 13

rhipidian, Antipathes 39

Rhipidipathes Milne Edwards \& Haime 1857 = Antipaihes 35

Rhizopsammia 134

Rhizosmilia 124

Rhizotrochus 129

Rhadarea Milne Edwards \& Haime $1849=$ Goniopora 72

Rhodocyathus Boume 1905 = Scolymia 98

Rhodopsammia Semper 1872 = ?Eupsammia 133

Rhombopsammia 82 richardi, Stichopathes Roule $1902=S$. filiformis 45

richmondia, Ornithoptera 10

richmondia, Omithoptera priamus = O. richmondia 10

riedeli, Troides 13

rigida, Antipathes 39

rigida, Hydnophora 100

rigida, Isophyllastrea 96

rigida, Mantipara 71

risei, Thalamaphyllia 125

rimosus, Lemiax 17

robillardi, Antipathes 39

robakaki, Mycedium 93

robusta, Acropora 67

rabusta, Crypthelia 53

robusta, Dendrophyllia 132

rabusta, Favia (Dana 1846) =F. abdita 106

robusta, Lepidotheca 56

robusta, Lobophyllia 97

rabusta, Oculina 92

rabusta, Podabacia Quelch 1886

= Sandalolitha rabusta [44] 88

rabusta, Sandalolitha 88

rabusta, Stenahelia 57

robustus, Stylaster 59

raissyana, Leplastrea Milne Edwards \& Haime 1848

= L. purpurea 108

rolandi, Cladopsammia 132

rosaceus, Stylaster 59

rosalindae, Distichapara 54

rasari, Acropora (Dana 1846) = ?A. langicyathus [65] 66 rosaria, Acropora 67

rosea, Achatinella 22

rosea, Distichopora Kent $1871=? D$. violacea [14] 54

rosea, Madrepora Pallas 1766 = Stylaster rosaceus [18] 59

roseus, Stylaster 59

rassiana, Paryphanta 24

rassiana, Pawelliphanta $=$ Paryphanta rassiana 24

rossoamericanus, Stylaster 59

rostrata, Enallopsammia 133

rosularia, Echinopora 105

rochschildi, Omithaptera 10

rothschildi, Schaenbergia = Omithaptera rothschildi 10

ratulosa, Favia 106

rotumana, Acropora (Gardiner 1899) = A. danai [65] 66

rotumana, Acropora 67

rotumana, Favia 106

ratundata, Favia 106

ratundata, Favices 107

rotundifolia, Oxysmilia 121

rotundoflora, Acanthastrea 95

rousseauii, Acropora Milne Edwards \& Haime = A. valida [65] 68

rawleyensis, Australamussa 95

rubeola, Culicia 89

rubescens, Crispatotrachus (Moseley 1881)

$$
\text { = Cyathoceras rubescens } 117
$$

nubescens, Cyathoceras 117

rubianus, Omithoptera victariae 11

rubra, Errina 55

rubrum, Flabellum 127

rubrum, Manamyces 128

nuepellii, Herpetolithus Leuckart 1841

= Crenactis echinata [44] 83

rufa, Faviles 107

ruficallis, Traides amphrysus 12

rugosa, Antipathella Thomson \& Simpson 1905

= Anripathes rugosa 39

rugosa, Antipathes 39

nugosa, Caryophyllia 115 
rugosa, Euphyllia Dana $1846=E$. glabrescens 119 rugasa, Favia Chevalier $1972=F$. manhai [98] 106 rugasa, Fungia Quelch $1886=F$. scrupasa [44] 86 rugasa, Pachyseris 81 rugasa, Stylaphora 64 rumphii, Cimhipathes 43 rus, Mantipora (Forgkt] ) = M. danae [65] 70 rus, Parizes 75 russelli, Favites 107 russelli, Plesiastrea Wells 1954 = Favites russelli 107 rustica, Platygyra (Dana 1846) $=P$. lamellinal $P$. daedalea [65] 111

nukyuensis, Platygyra 111

gukyuensis, Platygyra Yabe \& Sugiyama 1936 $=? P$. sinensis $[65] 111$

Sabinotrachus Duncan 1873 = Stephanocyathus 125 sabiuraensis, Catalaphyllia 116 saccharata, Parites 75 saccharatum, Lithadendrum von Martens 1902 = Distichapara caccinea [14] 54

saccula, Cirrhipathes 43

saccula, Stichapathes van Pesch 1914

= Cirrhipathes saccula 43

sagittatus, Troides helena 13

salebrosa, Plesiastrea Nemenzo = Favia helianthaides [79] 106

saliciformis, Tropidopathes 46

salicaides, Antipathes 39

salix, Antipathes 39

salix, Aphanipathes (Pourtalès 1880) = Antipathes salix 39 samarensis, Montipara 7I

samba, Strombus $=S$. gigas 24

sambaangensis, Fungia Vaughan $1906=F$. repanda [44] 85 samaa, Goniopara 73

samaaensis, Psammacara 78

samaaensis, Psammocara Hoffmeister 1925 $=$ ?P. nierstraszi $[50] 78$

samoensis, Acropora (Brook 1891) = 2A. humilis [50] 66 samaensis, Acropara 67

Sampson'a Riffleshell 18

sampsanii, Dysnomia = Epiablasma sampsanii 18

sampsonii, Epiablasma 18

sampsanii, Plagiala = Epiablasma sampsonii 18

Sandalolitha 88

sanguineus, Stylaster 59

sanguinalenta, Acrapora 67

samentosa, Acrapora 67

sarmentosa, Lepidopora 56

sarothamnoides, Antipathes 39

sarothamnoides, Aphanipathes Brook 1889 = Antipaches sarothamnoides 39

sarothrum, Antipathes 39

sarsiae, Caryaphyllia 115

satur, Lampsilis 19

satur, Lampsilis cardium = L. satur 19

satura, Lampsilis = L. satur 19

Savagliapsis Schultz 1896 = Antipathes 35

savigniensis, Psammocora 78

savignyana, Siderastrea 78

savignyi, Goniopara 73

saxea, Mantipara 71

scabiasus, Stylaster 59

scabra, Astreapara 69

scabra, Balanophyllia 131

scabra, Fungia 86

scabra, Leptaseris 80

scabricula, Merulina 100

scabricula, Mantipora 71 scabrasa, Balanophyllia I3!

scandens, Acropora (Klunzinger 1879) = A. pharaonis [64] 67

scandens, Acropara 67

scaphula, Placatrochides 128

Scapaphyllia 101

scheeri, Balanophyllia Durham 1962

= Rhizopsammia verrilli [25] 134

scheeri, Merulina 100

scherzeriana, Acropara (Brüggemann) = A. humilis [65] 66 schistica, Turbinaria 136

Schizoculina 92

Schizacyathus 130

Schizopaihes 44

schmitti, Acropora 67

schaenbergi, Omithoptera (Schaenbergia) $=0$. paradisea 10 scillaemorpha, Caryaphyllia 115

Sclerhelia 92

Sclerohelia Milne Edwarda \& Haime 1857 = Sclerhelia 92

Scleropages 2

Sclerophyllia Klunzinger 1979 = Cynarina 96

scabinosa, Caryophyllia II5

Scolymia 98

scoparia, Antipathes Lamarck $1816=$ A. dichotoma 36

scoparia, Bathypathes 42

scriptus, Holcatrochus 120

scrupasa, Fungia 86

scutaria, Fungia 86

sealarki, Antipathes 39

secale, Acrapara 67

secunda, Acropara (Dana 1846) = A. nobilis 67

secunda, Herpalitha Nemenzo = Fungia simplex [79] 86

securis, Acrapara (Dana 1846) = A. cuneata 66

seguenzae, Caryaphyllia 115

sekiseiensis, Acropora 67

selago, Acrapora 67

semiglabra, Cirrhipathes 43

semiglabra, Stichopathes 46

semilunaris, Porites Nemenzo = P. vaughani [79] 75

senaria, Madracis 62

senegalensis, Palycyathus 124

septata, Srylop.hara Gandiner $1898=S$. pistillata [65] 64 serailia, Cyphastrea 103

seriaua, Acrapora (Ehrenberg) = A. humilis [65] 66

seriata, Pavana Brüggemann $=P$. decussata $[65] 81$

Seriatopara 63

seriatus, Pliobathrus Broch 1942

= Phalangopara regularis [14] 56

serpens, Distichapora 54

serpentina, Dendraphyllia 132

serpuliforme, Desmophyllum Gravier 1915

= D. cristagalli [107] 118

semulata, Fungia Verrill $1864=F$. concinna [44] 85

setacea, Antipathes Gray $1860=$ Stichopathes gracilis 45

setacea, Stichopathes (Gray 1860) $=S$. gracilis 45

setacea var. occidentalis, Cirrhipathes Gray?

= Stichapathes gracilis 45

setchelli, Pocillopara 63

seiasa, Montipora 71

sewelli, Caryophyllia 115

scxcastatum, Flabellum 127

seychellensis, Goniastrea Milne Edwards \& Haime

$=G$. australensis [65] 107

seychellensis, Stichopathes 46

Shell, Curtis' Riffle I8

Shell, Green Riffle 18

Shell, Little Agate 21

Shell, Northern Riffle 18

Shell, Tan Riffle 19

Shell, Turbercuied Rime 18 
Shell, Turgid Riffle 18

Shell, Wabash Rifne 18

Shell, Yellow Riffle 18

sibogae, Antipathes 39

sibogae, Aphanipathes (van Pesch 1914)

= Antipathes sibogoe 39

sibogae, Flabellum 127

sibogae, Fungia van der Horst $1921=$ Cycloseris costulata 84 sibogae, Fungiacyachus 83

Sibopathes 44

Siderastrea 78

siderea, Siderastrea 78

Siderina Dana 1846 = Siderastrea 78

sieboldii, Cirmipathes Blainville $1834=? C$. anguina 42

sigmoides, Polyphyllia Ehrenberg $1834=$ P. talpino [44] 88

sillimaniani, Porites 75

similis, Cycloseris Nemenzo 1976

$=$ Fungia fralinae $[44,79] 85$

Simplastrea 93

simplex, Acanthastrea 95

simplex, Antipathes 39

simplex, Caryophyllia Duncan 1878

= Stenocyathus vermiformis [107] 130

simplex, Fungio (Gardiner 1905) = Cienactis crassa [44] 83

simplex, Gondineria 128

simplex, Gemmulatrochus Duncan 1873

$=$ Hoplangia durotrix [107] 120

simplex, Herperoglossa (Gardiner 1905) = Clenactis crasso 83

simplex, Herpolitha Gardiner 1905 = Chenactis crassa [44] 83

simplex, Parantipothes Schultze 1896 = Antipothes simplex 39

simplex, Plerogyra 123

simplex, Symphyllia 99

simplicitexta, Parasimplastrea 110

sinensis, Cimhipaches 43

sinensis, Cycloseris 84

sinensis, Favia (Milne Edwards \& Haime)

= Playgyra sinensis [65] 111

sinensis, Fungio (Milne Edwards \& Haime 1851)

$=$ Cycloseris sinensis 84

sinensis, Montipora Bemard $=M$. tuberculosa [65] 71

sinensis, Platygyra 111

sinensis, Turbinaria 136

singuloris, Acropora Nemenzo $=$ A. milleporo [79] 67

sinuosa, Calyptopora 52

sinuosa, Cycloseris Nemenzo 1983

$=$ Fungia concinna $[44],=F$. fungires $[79] 85$

sinuoso, Errina 55

sinuosa, Goniastrea (Dana 1846) $=$ G. pectinata 107

sinuosa, Isophyllia 97

sinuosa, Montipora 71

sinuosa, Mussa (Forskál) = Lobophyllia hemprichii 97

sinuosa, Plerogyra 123

sluizeri, Doderieinia van der Horst 1921

= Sandalolitha dentata [44] 88

smichi, Acropora (Brook) $=$ A. robusta $[65] 67$

smichi, Brochypelma 14

smithi, Culicia 89

smishi, Euachlus = Brochypelma smishi 14

smithii, Caryophyllia 115

Snail, Gillies' Land 23

Snail, Hochstetter's Land 23

Snail, Kauri 23

Snail, Manus Green Tree 23

Snail, Marchant'a Land 24

Snail, Oahu Tree 21

Snail, Oparara Land 23

Snail, Ross' Land 24

Snail, Speden'a Land 24

Snail, Supert Land 24
Snail, Travers' Land 24

Snail, Woodformed Land 24

socialis, Montipora Bernard $1897=M$. foveolata [65] 70

socialis, Thecopsammia 134

socrates, Troides haliphron 12

solanderi, Montipora 71

solanderi, Montipora Bernard = ?M. stellata [65] 71

Solenastrea 111

Solenosmilia 124

solida, Caryophyllia 115

solida, Leptastrea Milne Edwards \& Haime

$$
\text { = L. bottae [65] } 108
$$

solida, Leptoseris 80

solida, Pocillopora 63

solida, Porites 75

solidus, Leptopenus 82

solidus, Stylaster 59

solitoria, Astrangia 89

solitaryensis, Acropora 67

Soljania Pax 1955 = Hoplangia 120

solorensis, Cirrhipathes 43

solorensis, Srichopaches van Pesch 1914

$=$ Cirthipathes solorensis 43

somaliensis, Goniopora 73

somaliensis, Physogyra 123

somaliensis, Poriles 75

somaliensis, Porites Gravier $1911=$ ?P. lutea $[59,65] 75$

somenillei, Aphanipathes 41

somenillei, Cycloseris 84

somervillei, Fungio Gardiner $1909=$ Cycloseris somervillei 84

sonani, Troides magellanks 13

sonani, Troides $=T$. magellanis 13

sowerbyana, Achatinella 22

Spadefish 1

spoldingi, Achatinella 22

sparsa, Quadrula 20

spathula, Polyodon 1

spatula, Stylaster 59

speciosa, Anripathella Brook 1889 = Antipathes speciosa 39

speciosa, Antipathes 39

speciosa, Astrea Dana 1846 = Fovia speciosa [97] 106

speciosa, Favia 106

speciosa, Merulina Dana $1846=M$. ampliata [65] 100

speciosa, Pachyseris 81

spectabilis, Acropora (Brook) $=$ A. humilis $[65] 66$

spedeni, Paryphania 24

spedeni, Powelliphanta $=$ Paryphanta spedeni 24

spheniscus, Euphyllia Dana 1846

= Truncotoflabellum spheniscus [23] 129

spheniscus, Flabellum (Dana 1846)

= Truncaloflabellum spheniscus [23] 129

spheniscus, Truncaloflabellum 129

Sphenotrochus 124

spicifera, Acropora (Dans 1846) = ?A. hyacinthus [65] 66

spicifera, Acropora 67

spiessi, Stichopathes 46

spiloria, Troides helena 13

spinescens, Anripathes 39

spinifer, Fungia 86

spiniger, Stephanacyachus 125

spinosa, Anacropora 68

spinosa, Antipothes 39

spinosa, Inferiolobiala 55

spinasa, Pliobothrus 57

spinosa, Seriasopora 64

spinosocostatus, Trochocyathus 126

spinosum, Flabellum 127

spinosum, Hydradendrium Carter 1880

= Antipathes spinasa 39 
spinulosa, Echinopora 105

spinulosa, Euphylia Dana 1846 = Meandrina spinulosa 113 spinulosa, Meandrina 113

spiralis, Antipathes (Linnseus 1758)

= Cirrhipathes spiralis 43

spiralis, Cirmipathes 43

splendida, Acropora 67

splendida, Acropora Nemenzo 1967

= A. valenciennesi [79] 68

spangiosa, Alveopora 72

spongiosa, Errina Broch $1942=E$. antarctica [14] 54

spongiosa, Montipora 71

spongades, Mantipara 71

Sparadopara 57

spumasa, Montipora 71

squamosa, Acropora 67

squamosa, Antipathes 40

squamosa, Tridacna 17

squarrosa, Acropara (Ehrenberg 1834) = ?A. Loripes [65] 66 squarrosa, Acropara 67

squarrosa, Millepora 51

squarrosa, Pocillopora 63

squiresi, Caryophyllia 115

squiresi, Crispatotrochus (Caims 1979)

= Cyathoceras squiresi 117

squiresi, Cyathoceras 117

squiresi, Rhambopsammia 82

stabile, Truncaloflabellum 129

stabilis, Fungiacyathus 83

stoudingeri, Troides haliphron 12

steamsi, Paracyathus 122

stechowi, Antipathes 40

stechowi, Aphanipathes Pax 1932 = Antipathes stechawi 40

stejnegeri, Stylaster 59

stell(ul)atus, Baumeotrachus 114

stell(ul)atus, Stylaster 59

stellae, Astreapara Nemenzo = A. gracilis [79] 69

Stellangia Duchassaing \& Michelotti $1860=$ Astrangia 89

Stellapora 57

stellaris, Dichocoenia 113

stellaris, Haliglassa Ehrenberg 1834

= Herpolitha limax [44] 87

stellata, Culicia 89

stellata, Montipora 71

stellata, Psammocora 78

stellata, Seriatopora 64

stellata, Siderastrea 78

stellata, Stylaphora 64

stelligera, Favia 106

stellulata, Turbinaria 136

stellulatus, Deliocyathus 118

Stenocyathus 130

Stenohelia 57

stenopoma, Crypthelia 53

Stephanaria Verrill $1867=$ Psammacora 77

Stephanocoenia 61

Stephanocora Ehrenberg 1834 = Echinopora 104

Stephanocora Verrill $1866=$ Psammocora 77

Stephanocyathus 125

Stephanophyllia 82

Stephanopsammia Yabe \& Eguchi 1932 = Stephanophyllia 82

Stephanoseris Milne Edwards \& Haime 1851

= Heterocyathus 119

Stephanotrochus Moseley 1881 = Stephanocyathus 125

stephanus, Fungiacyachus 83

stephensani, Oryzotrochus 121

stephensoni, Porites 75

stephensoni, Turbinaria Crossland $=T$. stellulata [65] 136

Stichopathes 45 stigmataria, Acrapora 67

stimpsanii, Leptacyathus Pourtalès 1871

= Pepanocyathus australiensis [23] 122

stimpsonii, Peponocyathus (Pourtalès 1871)

$=P$. australiensis [23] 122

stimpsanii, Peponocyathus 122

swaddarti, Acropara 67

stokesi, Dichacoenia 113

stokesi, Goniopora 73

stokesi, Paracyathus 122

stokesi, Truncatoflabellum 129

stokesiana, Leptopsammia 134

stokesiana, Oulangia 90

stokesii, Flabellum Milne Edwards \& Haime 1848

= Truncatoflabellum stokesi [23] 129

striala, Acrapara 67

striala, Milleporo 51

striata, Quadrula 20

striatum, Desmaphyllum 118

stricta. Serialopara 64

stricus, Herpetalithus Dana $1846=$ Herpolitha limax [44] 87

strigosa, Antipathella Brook 1889 = Antipathes strigosa 40

strigosa, Antipathes 40

strigosa, Diploria 104

strigosa, Montipara Nemenzo $=$ M. stellata [79] 71

strigasa, Parantipathes 44

Strambus 24

studeri, Acropora 67

studeri, Crypthelia 53

studeri, Montipora 71

studeri, Porites 75

Sturgeon, Atlantic 1

Sturgeon, Common 1

Sturgeon, Shortnose 1

sturio, Acipenser 1

stutchburyi. Goniopora 73

Stylantheca 58

Stylaraea 76

Stylaster 58

stylifera, Errinopora 55

stylifera, Faviles 107

Stylacaeniella 61

Stylophara 64

stylasa, Montipara 71

subcostata, Lophelia Milne Edwards \& Haime 1850 = L. pertusa [107] 120

subdentata, Platygyra (Milne Edwards \& Haime 1849)

$$
=P \text {. Lamellina }[65,97] 111
$$

subglabra, Acrapara 68

subglabra, Echinophyllia 93

subpinnata, Antipathella (Ellis \& Solander 1786) = Antipathes subpinnata 40

subpinnata, Antipathes 40

subpinnata, Antipathes Gray $1857=$ A. wallastoni 40

subrepanda, Fungia Döderlein $1901=F$. scruposa [44] 86

subratunda, Fusconaia 19

subseriata, Stylaphora (Ehrenberg 1834)

$=S$. pistillata [65] 64

substellata, Polyphyllia Milne Edwards \& Haime 1851 $=$ P. novachibemiae [44] 88

subtilis, Montipora Bemand $1897=$ ?M. millepara [65] 71

subulato, Acropara 68

subulata, Seriatopora 64

subviolaceus, Stylaster 59

subviridis, Astya 52

suggesta, Astreapara 69

sulaensis, Troides hypalius 13

sulcata, Distichopora 54 
sulcara, Dysnomia 18

sulcaia, Montipora 71

sulcatus, Heterocyathus 119

sumatranus, Troides cuneifer 12

sumilonensis, Montipora 71

superba, Paryphania 24

superba, Powelliphanta = Paryphania superba 24

superficialis, Alveopono 72

superficialis, Psammocora 78

superfua, Porites 75

superstes, Stephanophyllia Ortmann 1888

= Letepsanmia formosissima [23] 82

suppressa, Porites Crossland $=P$. nigrescens [65] 75

surculosa, Acroporo (Dans 1846) = A. hyacinthus [65] 66

susanae, Galaxeo Nemenzo \& Ferraria $=G$. astreata [79] 9]

suteri, Kionotrochus 120

suvadivae, Cyphastrea Gardiner $=$ C. serailia [65] 103

suvadivae, Montipora 71

sverdrupi, Porites 75

Swainson, Achatinella 21

Swallowtail, Chinese Three-tailed 9

Swallowtail, Corsican 11

Swallowtail, Homerus 11

Swallowtail, Ludlow's Bhutan 9

Swallowtail, Luzon Pescock 11

Swallowail, Mansfield's Three-tailed 9

swifrii, Achatinella 23

symmetrica, Acropora (Brook) = ?A. cytherea [65] 66

symmetrica, Lepidopora 56

symmetricus, Fungiacyathus 83

symmetricus, Pliobothrus 57

Symphyllia 99

symphylloides, Pectinia 94

Synarea Verrill $1864=$ Porites 73

syringodes, Acropora (Brook 1893) = ?A. nana [50] 67

syringades, Acropora 68

Systemopora 59

tabulata, Astreopora 69

taeniolata, Acharinella 23

tagusensis, Tubasiraea 135

taisnei, Plerogyra Chevalier $1971=$ P. simplex 123

taiwanensis, Fungia 86

talpina, Fungia Lamarck 1801 = Polyphyllia talpina [44] 88

talpina, Polyphyllia 88

tanabensis, Cyphastrea 103

tanacetum, Antipathes 40

lanegashimensis, Acropora 68

Lannerensis, Polymyces 129

tarunggarensis, Omithoptera meridionalis 10

Taxipaines 46

tayam(a)i, Pseudosiderastrea 78

recomatensis, Lampsilis tampicoensis

$=$ Unio tampicoensis tecomatensis 20

tecomatersis, Unio tampicoensis 20

Teinopalpus 12

telegraphicus, Thalassiotrochus 134

venella, Acropora 68

ienella, Culicia 90

ienella, Gonioporo 73

tenella, Hydnophora Quelch $1886=H$. exesa $[65,97] 100$

ienella, Millepora 51

tenella, Millepora Ortmann $1892=M$. tenera 51

senella, Oculina 92

tenera, Millepora 51

tenisonwoodst, Stylaster 59

tenuescens, Desmophyllum 118

ienuicalyx, Trochocyathus 126

senuicaulis, Montipora 71 ienuicostatum, Mycedium Verrill $=M$. elephantotus [65] 93 senuiders, Fungia Quelch $1886=F$. scutario [44] 86 senuiders, Goniopora 73

ienuifolia, Agaricia 79

venuilamellosa, Coenopsammia 132

tenuis, Acropora 68

tenuis, Bathypathes 42

venuis, Coelastrea 102

venuis, Conopora Moseley $1879=$ C. laevis [14] 53

venuis, Cycloseris 84

venuis, Fungia Dana $1846=$ Cycloseris venuis 84

Lenuis, Leptoria (Dans 1846) = L. phrygia 108

ienuis, Leptoseris 80

tenuis, Lophelia Moseley 1881 = Madrepora tenuis []07] 92 renuis, Madrepora 92

renuis, Porites 75

tenuis, Sylaster Verrill $1864=S$. sanguineus [14] 59

tenuiseptata, Crypthelia 53

tenuispina, Antipathes 40

tenuispina, Parantipathes Silberfeld 1909

= Antipathes tenuispina 40

tenuissima, Montipora 71

tenuistylus, Lepidotheca 56

teres, Acropora 68

teres, Pectinia 94

tematersis, Antipathes 40

Tethocyathus 125

tetrasticha, Annipathes Pourtalès 1868

= Parantipathes tetrasticha 44

vetrasticha, Parantipathes 44

ietrastichopora, Conopora 53

levoroa, Tridacna 17

thaanumi, Achatinella 23

thaidina, Bhutanitis 9

Thalamophyllia 125

thalasse, Balanophyllia 131

Thalassiotrochus 134

Thalassiotrochus Milne Edwards $1861=$ ?Desmophyllum 118

thamnea, Antipathes 40

thamnoides, Antipathes 40

thamnoides, Aphanipathes Schultze 1896

$=$ Antipothes thamnoides 40

Thecopsammia 134

thestius, Troides oblongomaculatus 13

thiadina, Amandia = Bhutanitis thoidina 9

chomsoni, Troides aeacus 12

thouarsii, Flabellum 127

Thrypticotrochus 125

thurstoni, Acropora 68

thurstoni, Porites 75

thyoides, Antipathes Pourtalès 1880

= Aphanipathes thyoides 41

thyoides, Aphaniparhes 41

riara, Halomitra Verrill $1864=H$. pileus [44] 86

viburonensis, Paracyathus 122

Tichopora Quelch 1886 = Gonioporo 72

Tichoseris Quelch 1884 = Pavona 81

tiliata, Stenohelia 57

tintinnabulum, Bathypsammia 131

vithonus, Omithoptera 10

tithonus, Omithoptera tithonus 10

tithonus, Schoenbergia $=$ Omithoptera tithonus 10

tizardi, Acropora (Brook) = A. cerealis [65] 66

vizardi, Alveopora 72

Logianensis, Psammocoro Umbgrove $=P$. digitata [65] 77

corresiana, Pachyseris Vaughan $1918=P$. rugosa 81

tortuosa, Acropora 68

tartuosa, Millepora 52

tortuosa, Montipora (Dana 1846) $=$ M. digitata 70 
corulosa, Dysnomia torulosa

= Epiablasma torulosa torulosa 18

torulosa, Epioblasma torulosa 18

cortlosa, Plagiola torulosa = Epioblasma torulosa torulasa 18

Totana 3

toulai, Porites Vaughan $1919=$ P. baracoensis 74

Taxolasma 20

trabalis, Eurynia = Villosa trabalis 20

trabalis, Lampsilis = Villasa trabalis 20

trabalis, Micromya = Villosa trabalis 20

trabalis, Villosa 20

trabeculaka, Montipora 71

traceyi, Goniopora 73

traceyi, Goniopora Wells $1954=$ ?G. Lobata 73

Trachylapora Brook 1893 = Acropora 65

Trachyphyllia 112

Trachypora Verrill 1863 = Oxypora 94

iranslucens, Cirthipathes 43

transversa, Leptastrea 108

transversale, Flabellum 127

transversalis, Caryophyllia 115

trapezoideum, Truncatoflabellum 129

traversi, Paryphanta 24

traversi, Powelliphanta = Paryphanta traversi 24

Trematorrochus 125

iriangularis, Paraclavarina 100

Tridacna 16, 17

Tridacophyllia Blainville $1830=$ Pectinia 94

trihedralis, Alveopora Nemenzo = A. verrilliana [79] 72

trilinguis, Madrepora Boddaen 1768

= Hepolitha limax [44] 87

trimurata, Porites 75

trinitatis, Leptopsammia 134

tristicha, Parantipathes 44

tristis, Antipathella (Duchassaing 1870) = Antipathes tristis 40

tristis, Antipathes 40

tristis, Rhipidipathes Duchassaing $1870=$ Antipathes tristis 40

Trochocyathus 126

Trochopsammia 135

trogon, Trogonoptera brookiana 12

Trogonoptera 12

Troides 12, 13

trojana, Troganoptera 12

trojana, Troides = Trogonoptera trojana 12

trophostega, Crypthelia 53

Tropidacyathus 126

Tropidapathes 46

truncata, Culicia 90

Truncatoflabellum 129

Truncatoguynia 130

truncum, Truncatoflabellum 129

Tubastraea 135

Tubastrea Blainville $1830=$ Montastrea 109

tuberculata, Montipora (Lamarck) = ?M. danae [65] 70

tuberculosa, Acropora 68

tuberculosa, Montipora 71

tuberosa, Millepora 52

uberosa, Montipora 71

tuberosus, Orthonymus metanervus 20

tubicinaria, Acropora 68

ubifex, Mycedium (Dana 1846) $=$ M. elephantotus [65] 93

tubigera, Acropora 68

Tubipora 60

ubulata, Heliopora Pourtalès 1867

= Pliobothus mbulatus [18] 57

ubulatus, Pliobothrus 57

mubulifera, Favia Klunzinger $1879=F$. fanks [97] 106

mbulifera, Leptoseris Vaughan 1907

$=L$ hawaitensis $[35,65] 80$ tubuliferum, Flabellum 127

iubulosa, Acropora 68

tubulasa, Lophohelia Studer 1878

= Lophelia pertusa [107] 120

rumida, Acropora 68

numida, Caulastrea 102

turbida, Nemenzophyllia 121

turbida, Plerogyra 123

Turbinaria 135

urbinala, Acropora 68

turbinaliaides, Fungiacyathus 83

turgescens, Montipora 71

turgida, Achatinella 23

urrgida, Acropora 68

turgida, Euphyllia (Dana) = E. glabrescens [65] 119

curgidula, Dysnomia = Epiabiasma turgidula 18

urgidula, Epioblasma 18

aurgidula, Plagiala $=$ Epiablasma turgidula 18

eurtensis, Montipora 71

tuthilli, Flabellum 127

tydmani, Crispatotrochus 117

Tylopathes Brook 1889 = Antipathes $35-40$

Tylopora Brook 1893 = Acropora 65

cylostoma, Acropora Ehrenberg $=A$. horrida [65] 66

typhaon, Troides helena 13

typicus, Coelocyathus Sars 1857

$=$ Monomyces pgymaea [107] 128

typus, Rhizoirochus 129

uchiraensis, Monomyces Eguchi 1972

$=$ Rhizotrochus typus [23] 129

Ulangia Milne Edwards \& Haime $1857=$ Oulangia 90 ulex, Antipathes 40

Ulocyathus Sars $1851=$ Flabellum [23] 126

Ulophyllia Milne Edwards \& Haime 1857 = Oulophyllia 110

umbellifera, Porites 75

umbonata, Stenohelia 57

Undaria Oken $1815=$ Agaricia 79

undato. Agaricia ( 79

undata, Montipora 7I

undata, Turbinaria 136

undulata, Anripathes van Pesch 1914

= Aphanipathes undulata 41

undulata, Aphanipathes 41

undulata, Goniopora Nemenzo = G. somaliensis [79] 73

undulata, Porites (Klunzinger 1879) $=$ P. rus 75

undulatum, Lithophyllon 87

unifacilais, Conopora 53

Unio 20

uniserialis, Distichopora 54

urvillei, Plesiastrea Milne Edwards \& Haime

$=P$. versipora $[65] 111$

urvillianus, Omithoptera 10

urvillianus, Omithoptera priamus $=0$. urvillianus 10

valdiviae, Anripathes 40

valdiviae, Caryophyllia 115

valenciennesi, Acropora 68

valenciennesi, Favia 106

valenciennesi, Montastrea 109

valenciennesi, Oculina 92

valenciennesi, Pachyseris Milne Edwards \& Haime

$$
\text { = P. rugasa [65] } 81
$$

valenciennesii, Symphyllia 99

valida, Achatinella 23

valida, Acropora (Dana 1846) $=$ ?A. variabilis [64] 68

valida, Acropora 68

valida, Dasmosmilia 117

valida, Fungia 86 
valida, Fungia Verrill 1864

$=$ ?F. horridalscruposa $[44] 85186$

vandepolli, Troides 13

vandepolli, Troides vandepalli 13

vanderhorsti, Acropora Hoffmeister $1925=$ A. grandis 66

varia, Acropora Nemenzo = A. formosa [79] 66

varia, Goniastrea 107

variabile, Flabellum Semper 1872

= Truncatofabellum aculeatum [23] 129

variabile, Truncatoflabellum 129

variabilis, Acropora (Klunzinger 1879) = A. valida [65] 68

variabilis, Cirmipathes 43

variabilis, Peponocyathus Gravier 1915

$$
\text { =P. folliculus [23] } 122
$$

variabilis, Solenosmilia 124

variabilis, Srichopathes 46

variabilis, Tethacyathus 125

varians, Pavana 82

varicosa, Oculina 92

variegata, Dasmosmilia 117

variegatus, Fungiacyarhus 83

variolaris, Notophyllia 134

vascomarquesi, Crypthelia 53

vasiformis, Acropora (Brook) = A. clathrata [65] 66

Vasillum Tenison Woods $1879=$ Rhizotrochus [23] 129

Vaughanella 126

vaughani, Acropora 68

vaughani, Concentrotheca 116

vaughani, Cycloseris 84

vaughani, Endopachys Durham $1947=$ E. grayi [25] 133

vaughani, Flabellum 127

vaughani, Fungia Boschma 1923 = Cycloseris vaughani 84

vaughani, Menulina van der Horst $=M$. ampliasa 100

vaughani, Montipora 71

vaughani, Monripara Hoffmeister $1925=$ ?M. sociolis [50| 71

vaughani, Porites 75

vaughani, Psammocora 78

vaughani, Psammocora Yabe \& Sugiyama 1936 $=$ ?P. contigua $[65] 77$

velata, Dendrophyllia 132

veluto, Turbinaria Bernard $=T$. reniformis $[65] 136$

venosa, Montipora 72

venasa, Pavona 82

venusta, Pavona (Dana 1846) $=P$. cactus $[65] 81$

venustus, Citharocyathus Yabe \& Eguchi 1932

= Notocyarhus conicus [23] 121

venustus, Notocyathus 121

venustus, Stylaster 59

verconis, Trematatrochus 125

vermiculaia, Acropora Nemenzo = A. samentosa [79] 67

vermiformis, Coenocyathus Pourtalès 1868 = Stenocyathus vermiformis [107] 130

vermiformis, Stenocyathus 130

veroni, Favia 106

verreauxi, Culicia 90

verrilli, Montipara Vaughan 1907

$=M$. aequituberculata $[65] 70$

verrilli, Polycyathus 124

verrilli, Psammocora 78

verrilli, Rhizopsammia 134

verrilliana, Alveopora 72

verrilliana, Fungia Quelch $1886=$ F. scutaria [44] 86

verrillii, Stylaster 59

Verrillofungia Wells $1966=$ Fungia 85

vernucaria, Balanophyllia 131

verrucosa, Conopora 53

vermucosa, Montipora (Lamanck 1816) = ?M. monasteriata [65] 71

vermucosa, Montipora 72 verrucosa, Pacillopara 63

versipora, Plesiastrea 111

verticillata, Anripathes 40

verticillata, Aphanipathes Brook 1889 = Antipathes vericillata 40

venweyi, Acropora 68

venweyi, Platygyra 111

victoriae, Aethioptera = Omithoptera victoriae 11

victoriae, Omithoptera 11

victoriae, Omithoptera victariae 11

victoriae, Trachocyathus 126

victoriae, Truncatoflabellum 129

Villosa 19,20

viminalis, Antipathes 40

vincentinus, Australocyathus 114

vincentinus, Delsocyathus 118

viola, Sphenorrochus Gerth 1921

= Notocyathus conicus [23] 121

vialacea, Distichopora 54

violettae, Poriles 75

violellae, Poriles Nemenzo $=$ ?P. deformis 74

virens, Faviles (Dana 1846) $=F$, abdita 106

virens, Favites (Dana) $=$ F. abdita [65] 106

virescens, Lampsilis 19

virescens, Ligumia = Lampsilis virescens 19

virgala, Acropora (Dana 1846) = A. formosa 66

virgalo, Antipathes 40

virgaus, Trochacyathus 126

virginis, Cryptohelia Lindström 1877

= Stylaster complanatus [18] 58

virginis, Stylaster (Lindström 1871) $=S$. complanatus [18] 58

virgosa, Oculina 92

viridans, Achatinella 23

viridis, Alveopora 72

viridis, Goniopora 73

viridis, Porites Gardiner $1898=P$. lichen [65] 75

vistara, Troides amphrysiss 12

viriensis, Scalymia 99

vitrea, Javania (Alcock 1898) $=J$. cailleti [23] 128

vitreum, Desmophyllum Alcock 1898

= Javania cailleti [23] 128

viltata, Achatinella 23

vittatus, Paracyathus 122

vardermanni, Troides $=T$. dohertyi 12

vulpina, Acharinella 23

waigeuensis, Omithoptera tithonus 10

walkeri, Dysnomia = Epioblasma walkeri 19

walkeri, Epioblasma forentina $=$ E. walkeri 19

walkeri, Plagiola = Epioblasma walkeri 19

walkeri, Truncilla = Epioblasma walkeri 19

wallaceae, Acropora 68

washingtoni, Stenocyathus Cecchini 1914

$=S$. vermiformis [107] 130

waylandi, Poriles 75

weberi, Fungia van der Horst 1921

= Herpolitha limax [44] 87

weberi, Herpolitha (van der Horst 1921) = ?H. limax [44] 87

weberi, Herpolitha 87

wellingtoni, Rhizopsammia 134

wellsi, Balanophyllia 131

wellsi, Blastomussa 96

wellsi, Coscinaraea 76

wellsi, Erythrastrea 105

wellsi, Lophosmilia Duham \& Barnard 1952

$=$ Phyllangia consagensis [25] 90

wellsi. Physophyllia Nemenzo $1971=$ = ayleni [79| 95

wellsi, Polymyces 129 
wellsi, Scatymia 99

wellsi, Scalymia Laborel $1967=$ ?S. lacera 99

wellsi, Stylophora 64

wellsi, Trochocyathus Yabe \& Eguchi 1942

= Tropidocyathus Lessoni [23] 126

Wellsafungia ? = Fungia 85

Wellsophyllia 112

wettsteini, Rhizopsammia 134

willisae, Acropora 68

wilsoni, Symphyllia 99

wisseli, Favia 106

walfi, Parantipathes 44

wollastoni, Antipathes 40

wallastoni, Aphwnipathes Brook 1889

$=$ Antipathes wallasioni 40

woodjanesi, Pocillopora 63

waodst, Astrangia 89

woodsi, Crispatotrochus (Wells 1964)

= Cyathaceras waodsi 117

woodsi, Cyathaceras 117

worsleyi, Rhizotrochus Wood-Mason \& Alcock 1891

=R. typus [23] 129

xarifae, Pavana 82

xishaensis, Millepara 52
Xishasiderastrea Zou 1975 = Coeloseris 79

yabei, Leptaseris 80

yabei, Pavana Pillai \& Scheer = Lepinseris yabei [65] 80

yabei, Stenohelia 57

yacyamaensis, Euphyllia 119

yaeyamaensis, Goniastrea Eguchi \& Shiraj 1977

= Platygyra yacyamaensis 111

yacyamaensis, Plarygyra 111

yamanarii, Pavana 82

yongei, Acropara 68

yongei, Balanophyllia 131

yucaunensis, Distichopare 54

zamboi, Leptoseris Nemenzo $1971=$ L. papyracea $[35,79] 80$ zarhyncha, Errinopara 55

zeidleri, Paraconotrochus 121

zelandiae, Canocyathus 116

zelli, Australogyra 101

zelli, Platygyra Veron, Pichon \& Wijsman-Best 1977

= Australagyra zelli 101

Zoopilus 88

zoothallus, Antipathes 40

zopyras, Caryophyllia 115 


The UK Joint Nature Conservation Committee was established by the Environmental Protection Act 1990 "for the purposes of nature conservation, and fostering the understanding thereof" in Great Britain as a whole and outside Great Britain. It is a committee of the three country agencies (the Countryside Council for Wales, English Nature and Scottish Natural Heritage), together with independent members and representatives from Northem Ireland and the Countryside Commission, and is supported by a specialist staff. JNCC and the three country agencies carry forward duties previously undertaken by the Nature Conservancy Council.

JNCC's statutory responsibilities include:

- the establishment of common scientific standards;

- the undertaking and commissioning of research;

- advising Ministers on the development and implementation of policies for or affecting nature conservation for Great Britain as a whole or nature conservation outside Great Britain;

- the provision of advice and dissemination of knowledge to any persons about nature conservation.

JNCC also has the UK responsibility for European and intemational matters affecting nature conservation.

Details of publications produced by JNCC are available from JNCC, Monkstone House, City Road, Peterborough PE1 1JY. 